\title{
UNIVERSITY OF GÖTTINGEN
}

DOCTORAL DISSERTATION

\section{Preferential flow dynamics in the vadose zone of fractured and fractured-porous media: Development of a parallelized multi-scale Smoothed Particle Hydrodynamics model}

\author{
Dissertation \\ for the award of the degree \\ Doctor rerum naturalium (Dr.rer.nat.) \\ of the Georg-August-Universität Göttingen \\ within the doctoral program Geoscience \\ of the Georg-August University School of Science (GAUSS) \\ in the \\ Fractured Rock Hydrogeology Group \\ Department of Applied Geology
}

Submitted by

Elena SHIGORINA

from Tomsk, Russia

Göttingen, 2019 


\section{Thesis Committee:}

Prof. Dr. Martin Sauter (Department of Applied Geology, Georg-August University of Göttingen),

Prof. Dr. Thomas Ptak (Department of Applied Geology, Georg-August University of Göttingen),

Dr. Jannes Kordilla (Department of Applied Geology, Georg-August University of Göttingen),

Prof. Dr. Andreas Tilgner (Institute of Geophysics, Georg-August University of Göttingen),

Prof. Dr. Insa Neuweiler (Institute of Fluid Mechanics and Environmental Physics in Civil Engineering, Leibniz University of Hannover),

Prof. Dr. Hongbiao Gu (Department of Earthquake Science, Institute of Disaster Prevention, China).

Members of the Examination Board:

First Reviewer: Dr. Jannes Kordilla (Department of Applied Geology, GeorgAugust University of Göttingen).

Second Reviewer: Prof. Dr. Andreas Tilgner (Institute of Geophysics, GeorgAugust University of Göttingen),

Date of the oral examination: 19 February, 2020 


\section{Declaration of Authorship}

I, Elena SHIGORINA, declare that this thesis titled, "Preferential flow dynamics in the vadose zone of fractured and fractured-porous media: Development of a parallelized multi-scale Smoothed Particle Hydrodynamics model" and the work presented in it are my own. I confirm that:

- This work was done wholly or mainly while in candidature for a research degree at this University.

- Where any part of this thesis has previously been submitted for a degree or any other qualification at this University or any other institution, this has been clearly stated.

- Where I have consulted the published work of others, this is always clearly attributed.

- Where I have quoted from the work of others, the source is always given. With the exception of such quotations, this thesis is entirely my own work.

- I have acknowledged all main sources of help.

- Where the thesis is based on work done by myself jointly with others, I have made clear exactly what was done by others and what I have contributed myself.

Signed:

Date: 

"You never change things by fighting the existing reality. To change something, build a new model that makes the existing model obsolete."

Buckminster Fuller 



\title{
UNIVERSITY OF GÖTTINGEN
}

\author{
Abstract \\ Department of Applied Geology

\section{Preferential flow dynamics in the vadose zone of fractured and fractured-porous media: Development of a parallelized multi-scale Smoothed Particle Hydrodynamics model}

\author{
by Elena SHIGORINA
}

The main objective of this thesis is the development of a smoothed particle hydrodynamics (SPH) model to study preferential flow dynamics in partially saturated porous-fractured media at core- and micro scale. The research is focused on the numerical investigation of preferential flow in the vadose zone, including the estimation of geometrical and hydraulic properties of the subsurface, such as wettability, surface roughness, hydraulic conductivity, and infiltration rate, which are influencing the infiltration dynamics under saturated, unsaturated and partially saturated conditions. In the first part of the thesis the simulation results of sessile and transient droplets on hydrophobic and hydrophilic structured rough surfaces are presented. The results show that the effective static contact angles of Cassie and Wenzel droplets on a rough surface are greater than the corresponding micro scale static contact angles. As a result, micro scale hydrophobic rough surfaces can also exhibit effective hydrophobic behavior. This study considers, as well, the impact of the roughness orientation (i.e., an anisotropic roughness) and surface inclination on droplet flow velocities. The results show that droplet flow velocities are lower if the surface roughness is oriented perpendicular to the flow direction. The second part deals with the investigation of infiltration instabilities in smooth and rough fractures, focusing on the influence of roughness and injection rate on fluid flow modes and flow velocity. Both the rough and smooth fractures exhibit flow instabilities, fingering, and intermittent flow regimes for low infiltration rates. A flat fluid front is achieved when the flux supplied to a fracture is larger than the gravitationally driven saturated flux. An increase in roughness decreases the flow velocity and increases the standard deviation of velocity. This is caused by a higher likelihood of flow discontinuities in the form of fingering and/or snapping rivulets. The scaling of specific discharge with normalized finger velocity and the relationship between fingertip length and scaled finger velocity are in a good agreement with experimental results. The final part is devoted to a newly developed fully-coupled multi-scale SPH model, which considers flow through a porous matrix governed by the volume-effective Richards equation, and discrete free-surface flows governed by the Navier-Stokes equation. Inflow dynamics from the fracture into the porous matrix are realized by an efficient particle removal algorithm and a virtual water redistribution formulation in order to enforce mass and momentum conservation. The model validation is carried out via comparison to a FEM model (COMSOL) for the Richards based flow dynamics and laboratory experiments to cover more complex cases of free-surface flow dynamics and matrix infiltration. The developed model is employed to investigate preferential flow dynamics at a fracture-matrix interface. Simulation results show that preferential flow occurs in most cases simultaneously with the diffuse flow. Depending on the infiltration rate, the ponding effect can be dominant until the matrix saturation is high enough to activate fracture flow. For extremely high infiltration rates, fracture flow is dominant and ponding occurs when the fracture space is fully saturated. Next, the model is employed to simulate infiltration dynamics in rough fractures embedded in impermeable and permeable porous walls for different infiltration rates. The simulation results indicate a delay in arrival times for fractures with permeable walls in comparison to impermeable fractures, especially for small infiltration rates. Here, for higher infiltration rates, water flows rapidly to the bottom of the fracture without any significant delay in arrival time. 



\section{Acknowledgements}

I would like to express my gratitude to everyone who has been supporting me financially, intellectually or emotionally throughout my doctoral research at University of Göttingen. I was lucky enough to receive full support from my supervisor Dr. Jannes Kordilla. I am thankful not only for his intellectual support and great scientific ideas, but also for his understanding and extension of my working contract during my parental leave. Without his help it would be impossible to complete this research.

I would like to thank all committee members, especially, Prof. Dr. Martin Sauter, Prof. Dr. Ptak, Prof. Dr. Neuweiler, Prof. Dr. Tilgner, Prof. Dr. Gu for reading my thesis and for taking part in my defense.

My particular thanks go to Prof. Dr. Alexandre Tartakovsky for his supervision during my visit at the Pacific Northwest National Laboratory (PNNL). His optimistic and friendly personality helped me not only to do my research but also to enjoy my stay in Richland.

I am grateful for the financial support by the German Research Foundation (DFG) for my work in Göttingen and by German Academic Exchange Service (DAAD) for my visit abroad.

I am grateful for the help of my friends and colleagues at the Department of Applied Geology. Beka is thanked for her administrative support and the preparation of documents and contracts. I appreciate the kindness of my colleagues whom I met close to the end of my doctoral research. Special thanks to Hongbiao for his interest in my research, to Miriam for her help in taking care of my son, and to Florian for his laboratory experiments.

Last but not least, my deepest thanks go to my Family, especially to Lars and Alessandro for always believing in me, for their continuous love and support in my decisions, without whom I could not have made it here. I also thank my parents for raising me and giving me a chance to get an excellent school education. Special thanks go to my elder brother Alexander for helping with my homework at school and for taking care of me when I was little, and to my elder sisters Galina and Lubov for being an example for me in life. I also thank my aunt Katharina for keeping me in optimistic mood no matter what. 



\section{Contents}

Declaration of Authorship iii

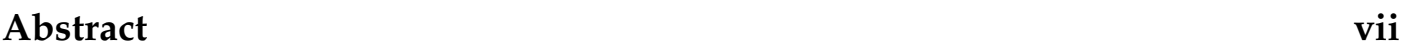

$\begin{array}{ll}\text { Acknowledgements } & \text { ix }\end{array}$

1 Introduction 1

1.1 Motivation . . . . . . . . . . . . . . . . 1

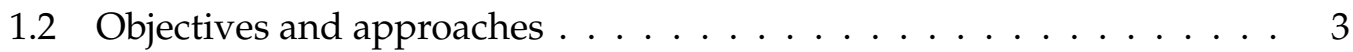

1.3 Format of the thesis . . . . . . . . . . . . . . 3

2 General overview $\quad 7$

2.1 Fractured porous media . . . . . . . . . . . . . 7

2.2 Characterization of flow in unsaturated fractured porous media . . . . 7

2.2.1 Experimental approaches . . . . . . . . . . . . 8

2.2 .2 Theoretical approaches . . . . . . . . . . . . . . . 9

2.2.3 Numerical approaches . . . . . . . . . . . . . . . . . . . . 10

3 Multiscale PF-SPH model 19

3.1 PF-SPH method and governing equations . . . . . . . . . . . . . . . 19

3.2 Simulation algorithm . . . . . . . . . . . . . . . 22

$\begin{array}{lll}4 & \text { SPH study of the roughness effect on contact angle and droplet flow } & 27\end{array}$

4.1 Introduction . . . . . . . . . . . . . . . 27

4.2 Governing equations and PF-SPH method . . . . . . . . . . . . . . . 29

4.3 Model parametrization and verification . . . . . . . . . . . . . 31

4.3 .1 Surface tension . . . . . . . . . . . . . . . . . . 31

4.3.2 Static contact angles on smooth surface . . . . . . . . . . . 32

4.3.3 Dynamic contact angles on smooth surface . . . . . . . . . . . 33

4.4 Wenzel and Cassie droplets on rough solid surfaces . . . . . . . . . . . 34

4.5 Effective contact angles of droplets on rough microscopically hydrophobic surfaces . . . . . . . . . . . . . . . . . . . . . 35

4.5.1 Rectangular and dual-rectangular surfaces . . . . . . . . . 36

4.5.2 Sinusoidal and dual-sinusoidal surfaces . . . . . . . . . . . . 37

4.6 Effective contact angles of droplets on rough microscopically hydrophilic surfaces . . . . . . . . . . . . . . . . . . . 38

4.7 Dimensionless analysis of effective static contact angles . . . . . . . . . 39

4.8 The effect of resolution on effective static contact angle . . . . . . . . . 40

4.9 Transitions between Cassie and Wenzel states . . . . . . . . . . . . . . . 41

4.10 Droplet flow on rough surfaces . . . . . . . . . . . . . . . . 46

4.11 Conclusion . . . . . . . . . . . . . . . . . . 48 
5 Investigation of gravity-driven infiltration instabilities in smooth and rough fractures 53

5.1 Introduction . . . . . . . . . . . . . . . . . 54

5.2 Governing equations and the PF-SPH method . . . . . . . . . . . 56

5.3 Gravity-driven flow instability in initially dry fractures . . . . . . . . . 59

5.3 .1 Flow in smooth vertical fractures . . . . . . . . . . . . . 60

5.3 .2 Flow in rough vertical fractures . . . . . . . . . . . . . . 62

5.3.3 Comparison with analog experiments . . . . . . . . . 63

5.4 Discussion . . . . . . . . . . . . . . . . . 67

5.5 Conclusion . . . . . . . . . . . . . . . . . . . . . 69

6 Multiscale SPH model development for simulating preferential flow dy$\begin{array}{ll}\text { namics in fractured porous media } & 75\end{array}$

6.1 Introduction . . . . . . . . . . . . . . . 76

6.2 Governing Equations and the PF-SPH method . . . . . . . . . . . 77

6.3 Model Validation . . . . . . . . . . . . . . . . . . . . . . . . 81

6.3 .1 Constant pressure head boundary . . . . . . . . . . 81

6.3 .2 Drop imbibition . . . . . . . . . . . . . . . . . 82

Experimental and simulation setup . . . . . . . . 82

Parameter estimation . . . . . . . . . . . . . . . . . 84

Results . . . . . . . . . . . . . . . . . . 8 85

6.3.3 Water infiltration into a sandstone . . . . . . . . . . . . . 85

6.3.4 Flow on the fracture wall and in the adjacent matrix in sandstone 86

6.4 Preferential flow dynamics at a fracture-matrix interface . . . . . . 88

6.5 Unsaturated fractured porous medium flow . . . . . . . . . . . . . . . 89

6.6 Discussion . . . . . . . . . . . . . . . . 93

6.7 Conclusion . . . . . . . . . . . . . . . . 93

$\begin{array}{llr}7 & \text { Summary and conclusion } & 99\end{array}$

7.1 Main results . . . . . . . . . . . . . . . . . . . . . . 999

7.1.1 The effect of roughness on contact angle and droplet flow . . . 99

7.1.2 Infiltration instabilities in smooth and rough fractures . . . . 100

7.1.3 The development of multiscale SPH model for flow in fracturedporous media . . . . . . . . . . . . . . 100

7.2 General conclusion . . . . . . . . . . . . . . . . . 100

7.3 Suggestions for further research . . . . . . . . . . . . . . . 101

7.3.1 Adaptive resolution . . . . . . . . . . . . . . . . . 101

7.3.2 Implementation of an evapotranspiration equation $\ldots . . .102$

7.3.3 Particle addition algorithm . . . . . . . . . . . . 102

7.3.4 Multiphase flow simulations . . . . . . . . . . . . . . 102

7.3.5 Reactive transport . . . . . . . . . . . . . . . . . 102 


\section{List of Figures}

1.1 Geometrical and hydraulic subsurface properties influencing infiltration dynamics. . . . . . . . . . . . . . . . .

2.1 Scale hierarchy of fractured rocks in the unsaturated zone. Modified from Faybishenko et al. (2003) . . . . . . . . . . . . . . . . . . . . 8

2.2 Scale hierarchy of numerical techniques used in hydrogeology. MD: molecular dynamics; DPD: dissipative particle dynamics; SDPD: smoothed dissipative particle dynamics; SPH: moothed particle hydrodynamics. Information compiled from Dzwinel et al. $(1999,2006)$; Kulkarni et al. (2013) . . . . . . . . . . . . . . . . . . . . . . 11

3.1 Kernel $W$ with a circular support domain of length $h$ and value $W\left(r_{i j}, h\right)$ between particles $i$ and $j$ at a distance $r_{i j} \ldots \ldots \ldots 20 \ldots \ldots$

3.2 Simulation algorithm within the LAMMPS class hierarchy. . . . . . . . 23

4.1 Pressure for various droplet sizes. . . . . . . . . . . . . . . . . . 32

4.2 Static contact angle measurements. Here, only the droplet cross-section in the $x y$-plane is shown. . . . . . . . . . . . . . . . 33

4.3 Static contact angles for different solid-fluid interaction strengths $s_{\mathrm{sf}}$. . 33

4.4 Model verification with respect to Tanner's law: height of the droplet as a function of time. The inset shows the droplet spreading on a horizontal surface. . . . . . . . . . . . . . . . . . .

4.5 Cox-Voinov relationship for receding contact angles. The inset shows the simulation results of a plate withdrawal from a pool of liquid $(x=$ $25 \mathrm{~mm}, y=10 \mathrm{~mm}) . \ldots \ldots \ldots \ldots \ldots \ldots$

4.6 Different states of droplets depending on wetting conditions (left to right): Cassie state, Wenzel state, and Cassie-Wenzel state. . . . . . . . 35

4.7 Surface parameters (four types, from left to right): (1) for rectangular surface - height $H$ and width $l$ of a bar; $d$ - distance between bars; (2) for dual-rectangular surface - height $H$ and $l$ width of a block, $d$ - distance between blocks; (3) for sinusoidal surface - period $T$ and magnitude $A$ of a sinusoidal function in the $x$ direction; (4) for dualsinusoidal surface - period $T$ and magnitude $A$ of a sinusoidal function in the $x$ and $z$ directions. . . . . . . . . . . . . .

4.8 Static contact angles of droplets on hydrophobic rectangular $(\mathrm{a}-\mathrm{c})$ and dual-rectangular $(\mathrm{d}-\mathrm{f})$ surfaces. Surface parameters are: $d=0.2 \mathrm{~mm}$, $l=0.15 \mathrm{~mm}(\mathrm{a}, \mathrm{d}) ; d=0.25 \mathrm{~mm}, l=0.2 \mathrm{~mm}(\mathrm{~b}, \mathrm{e}) ; d=0.25 \mathrm{~mm}$, $l=0.25 \mathrm{~mm}(\mathrm{c}, \mathrm{f}) ; H=0.2 \mathrm{~mm}$ for all types of surfaces. . . . . . . . 36

4.9 Static contact angles of droplets on hydrophobic sinusoidal $(\mathrm{a}-\mathrm{c})$ and dual-sinusoidal (d - f) surfaces. Surface parameters are: $A=0.2 \mathrm{~mm}$, $\mathrm{T}=0.2 \mathrm{~mm}(\mathrm{a}, \mathrm{d}) ; A=0.2 \mathrm{~mm}, T=0.25 \mathrm{~mm}(\mathrm{~b}, \mathrm{e}) ; A=0.2 \mathrm{~mm}$, $T=0.3 \mathrm{~mm}(\mathrm{c}, \mathrm{f}) \ldots \ldots \ldots \ldots \ldots \ldots \ldots \ldots$ 
4.10 Static contact angles of droplets on hydrophilic rectangular $(\mathrm{a}-\mathrm{c})$ and dual-rectangular $(\mathrm{d}-\mathrm{f})$ surfaces. Surface parameters are: $d=0.2 \mathrm{~mm}$, $l=0.15 \mathrm{~mm}(\mathrm{a}, \mathrm{d}) ; d=0.25 \mathrm{~mm}, l=0.2 \mathrm{~mm}(\mathrm{~b}, \mathrm{e}) ; d=0.25 \mathrm{~mm}$, $l=0.25 \mathrm{~mm}(\mathrm{c}, \mathrm{f}) ; H=0.2 \mathrm{~mm}$ for all types of surfaces. . . . . . . 38

4.11 Static contact angles of droplets on hydrophilic sinusoidal $(\mathrm{a}-\mathrm{c})$ and dual-sinusoidal ( $\mathrm{d}-\mathrm{f})$ surfaces. Surface parameters are: $A=0.2 \mathrm{~mm}$, $\mathrm{T}=0.2 \mathrm{~mm}(\mathrm{a}, \mathrm{d}) ; A=0.2 \mathrm{~mm}, T=0.25 \mathrm{~mm}(\mathrm{~b}, \mathrm{e}) ; A=0.2 \mathrm{~mm}$,

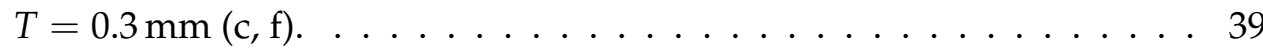

4.12 Effective static contact angles $\theta_{\text {eff }}^{x}$ and $\theta_{\text {eff }}^{z}$ for scaling ratio $\lambda$ between $3.5 \times 10^{-3}$ and $8.5 \times 10^{-3}$ for hydrophobic rectangular and dual-rectangular (a), hydrophilic rectangular and dual-rectangular (b), hydrophobic sinusoidal and dual-sinusoidal (c), and hydrophilic sinusoidal and dual-sinusoidal (d) surfaces. Solid symbols represent droplets in a Cassie state, and open symbols represent droplets in a Wenzel state. Symbols "plus" and "cross" represent a droplet in a Cassie-Wenzel state. 41

4.13 The effective static contact angle difference of droplets on rough hydrophobic and hydrophilic surfaces. Red: hydrophilic; blue: hydrophobic; square: rectangular surface; circle: sinusoidal surface; solid: dual surface; empty: non-dual surface. . . . . . . . . . . . . . . . . . 42

4.14 Comparison of effective contact angles on a fine-roughness dual-rectangular surface obtained from a high-resolution (particle spacing $2.5 \times 10^{-5} \mathrm{~mm}$; $\theta_{\mathrm{eff}}^{x}=149.36^{\circ} ; \theta_{\mathrm{eff}}^{z}=150.28^{\circ}$ ) and a low-resolution (particle spacing $5 \times 10^{-5} \mathrm{~mm} ; \theta_{\text {eff }}^{x}=151.52^{\circ} ; \theta_{\text {eff }}^{z}=150.84^{\circ}$ ) simulation. Green particles: solid surface; red particles: low resolution; blue particles: high resolution. . . . . . . . . . . . . . . . . . . 42

4.15 Droplets on a fine-roughness dual-rectangular surface. Equilibrated radii of droplets are $1.5,1.3,1.11,0.92,0.54$, and $0.44 \mathrm{~mm}$. . . . . . . 43

4.16 Pinning effect of droplets on a fine-roughness dual-rectangular surface. Equilibrated radii of droplets are 1.11, 1.3, and $1.5 \mathrm{~mm}$. . . . . . . 44

4.17 Cassie-to-Wenzel transition based on critical capillary pressure and internal pressures of droplets with $R_{\text {eq }}$ ranging from $0.44 \mathrm{~mm}$ to $1.5 \mathrm{~mm}$ for a fine-roughness dual-rectangular surface. . . . . . . . . . . . . 44

4.18 Cassie-to-Wenzel transition based on critical capillary pressures and internal pressures of droplets ranging from $97.00 \mathrm{~Pa}$ to $330.68 \mathrm{~Pa}$ (corresponding $R_{\text {eq }}$ are ranging from $1.5 \mathrm{~mm}$ to $0.44 \mathrm{~mm}$ ) for fine-, medium, and coarse-roughness dual-rectangular surfaces. Open symbols: Wenzel regime; filled symbols: Cassie regime. . . . . . . . . . . . . .

4.19 Droplet states depending on initial conditions: (a) droplets with $R_{\mathrm{eq}}=$ $0.64 \mathrm{~mm}$ are brought into contact with a rough surface; (b) droplets with $R_{\text {eq }}=0.64 \mathrm{~mm}$ are dropped from $1.3 \mathrm{~mm}$ height; (c) droplets with $R_{\mathrm{eq}}=1.3 \mathrm{~mm}$ are brought into contact with a rough surface; (d) droplets with $R_{\mathrm{eq}}=1.3 \mathrm{~mm}$ are dropped from $1.75 \mathrm{~mm}$ height. . . . . . 45

4.20 Hydrophobic and hydrophilic droplets flowing on a rough rectangular surface with rectangular bars oriented parallel to the flow direction (a) and rectangular bars oriented perpendicular to the flow direction (b) at the time step $t=50000(46.296 \mathrm{~ms})$. Surface inclination angle is $\alpha=90^{\circ} \ldots \ldots \ldots \ldots \ldots \ldots \ldots \ldots$ 
4.21 Dimensionless scaling for smooth and rough hydrophobic and hydrophilic surfaces with different orientations of roughness relative to the droplet flow direction. (par.) - flow parallel to the orientation of the bars, (perp.) - flow perpendicular to the orientation of bars. The surface inclination angles $\alpha$ are $10^{\circ}, 20^{\circ}, 30^{\circ}, 40^{\circ}, 50^{\circ}, 60^{\circ}, 70^{\circ}, 80^{\circ}$ and $90^{\circ}$.

5.1 Fracture configurations: smooth (left); rough with $\zeta=0.75$ and $\Delta=$ $10 \mathrm{~mm}$ (middle); rough with $\zeta=0.75$ and $\Delta=20 \mathrm{~mm}$ (right). . . . . . 60

5.2 Flow inside smooth fractures (six flow rates, from left to right): (a) $Q=4 \times 10^{-6} \mathrm{~m}^{3} / \mathrm{s}, t=1.44 \mathrm{~s}$; (b) $Q=8 \times 10^{-6} \mathrm{~m}^{3} / \mathrm{s}, t=1.07 \mathrm{~s}$; (c) $Q=2 \times 10^{-5} \mathrm{~m}^{3} / \mathrm{s}, t=0.74 \mathrm{~s}$; (d) $Q=6 \times 10^{-5} \mathrm{~m}^{3} / \mathrm{s}, t=0.46 \mathrm{~s}$; (e) $Q=1 \times 10^{-4} \mathrm{~m}^{3} / \mathrm{s}, t=0.40 \mathrm{~s}$; (f) $Q=5.06 \times 10^{-4} \mathrm{~m}^{3} / \mathrm{s}, t=0.11 \mathrm{~s} .$.

5.3 Flow inside rough fractures with $\Delta=10.0 \mathrm{~mm}$ (six flow rates, from left to right): (a) $Q=4 \times 10^{-6} \mathrm{~m}^{3} / \mathrm{s}, t=1.65 \mathrm{~s}$; (b) $Q=8 \times 10^{-6} \mathrm{~m}^{3} / \mathrm{s}$, $t=1.19 \mathrm{~s}$; (c) $Q=2 \times 10^{-5} \mathrm{~m}^{3} / \mathrm{s}, t=0.74 \mathrm{~s}$; (d) $Q=6 \times 10^{-5} \mathrm{~m}^{3} / \mathrm{s}$, $t=0.46 \mathrm{~s}$; (e) $Q=1 \times 10^{-4} \mathrm{~m}^{3} / \mathrm{s}, t=0.40 \mathrm{~s}$; (f) $Q=5.06 \times 10^{-4} \mathrm{~m}^{3} / \mathrm{s}$,

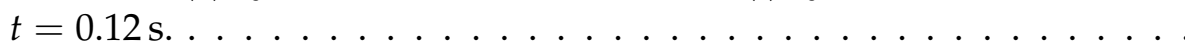

5.4 Flow inside rough fractures with $\Delta=20.0 \mathrm{~mm}$ (six flow rates, from left to right): (a) $Q=4 \times 10^{-6} \mathrm{~m}^{3} / \mathrm{s}, t=1.65 \mathrm{~s}$; (b) $Q=8 \times 10^{-6} \mathrm{~m}^{3} / \mathrm{s}$, $t=1.19 \mathrm{~s}$; (c) $Q=2 \times 10^{-5} \mathrm{~m}^{3} / \mathrm{s}, t=0.74 \mathrm{~s}$; (d) $Q=6 \times 10^{-5} \mathrm{~m}^{3} / \mathrm{s}$, $t=0.46 \mathrm{~s}$; (e) $Q=1 \times 10^{-4} \mathrm{~m}^{3} / \mathrm{s}, t=0.40 \mathrm{~s}$; (f) $Q=5.06 \times 10^{-4} \mathrm{~m}^{3} / \mathrm{s}$,

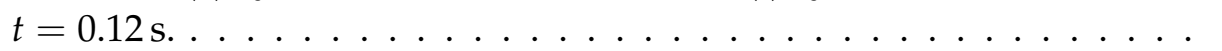

5.5 Average flow velocities in smooth and rough fractures for different infiltration rates. . . . . . . . . . . . . . . . . . . . . . 64

5.6 Standard deviations of flow velocities in smooth and rough fractures for different infiltration rates. . . . . . . . . . . . . . . . . 65

5.7 Comparison of the normalized averaged flow velocity from PF-SPH simulations with the normalized averaged velocity $\bar{v}_{\mathrm{f}}$ of individual fingers from experiments (Nicholl and Glass; 2005, Fig. 27). (black line) $p=0.5$, (grey line) $p=0.53 \ldots \ldots \ldots \ldots \ldots$

5.8 Comparison of the scaled fingertip length $\left(L_{\text {tip }} \cos \varphi\right)$ as a function of scaled finger velocity $\hat{v}=v / K_{s} k_{r} \cos \varphi$ for simulations and experiment from Nicholl and Glass (2005). For the analytical solutions $\bar{L}_{t i p}=2.85 \mathrm{~cm}$ and $\epsilon=0.1$ (experiments) and $\bar{L}_{t i p}=1.0 \mathrm{~cm}$ and $\epsilon=0.1$ (simulations). . . . . . . . . . . . . .

6.1 Particle removal algorithm.

6.2 Pressure head distributions for a vertical column with constant pressure head boundary at different times: (a) $t_{0}=0 \mathrm{~h}$, (b) $t_{1}=1 \mathrm{~h}$, (c) $t_{2}=6 \mathrm{~h}$, and $(\mathrm{d}) t_{3}=16 \mathrm{~h} \ldots \ldots \ldots \ldots \ldots$

6.3 Comparison of pressure heads at different times for SPH and COMSOL models. . . . . . . . . . . . . . . . . . .

6.4 The experimental (top) and simulation (bottom) results of droplet imbibition at different times: (a) $t_{0}=-0.004 \mathrm{~s}$, (b) $t_{1}=0.396 \mathrm{~s}$, (c) $t_{2}=1.836 \mathrm{~s}$, and $(\mathrm{d}) t_{3}=2.676 \mathrm{~s} . \ldots \ldots \ldots . \ldots \ldots$

6.5 Porosimetry of Seeberger Sandstone (Sustrate; 2017). . . . . . . . . . . . 84

6.6 The dependence of interaction forces between solid and fluid particles $s_{s f}$ on time $t$, the stabilization time $t_{e q}=0.4 s \ldots \ldots \ldots \ldots$

6.7 Comparison of the experimental (top) and simulation (bottom) results of $4.0 \mathrm{~mL}$ water infiltrating into a sandstone at different time intervals: (a) $t_{1}=3 \mathrm{~s}$; (b) $t_{2}=16 \mathrm{~s}$; (c) $t_{3}=30 \mathrm{~s}$; (d) $t_{4}=50 \mathrm{~s}$; (e) $t_{5}=100 \mathrm{~s} \ldots \ldots$ 
6.8 Experimental and simulation measurements of water level above the sandstone during infiltration.

6.9 Comparison of experimental (top) and simulation (bottom) results of free-surface flows on a porous sandstone at different time intervals: (a) $t_{1}=5 \mathrm{~s} ;$ (b) $t_{2}=22 \mathrm{~s}$; (c) $t_{3}=44 \mathrm{~s}$; (d) $t_{4}=66 \mathrm{~s}$; (e) $t_{5}=110 \mathrm{~s} \ldots \ldots 88$

6.10 Experimental measurements and simulation results of water outflow mass. . . . . . . . . . . . . . . . . . 88

6.11 Infiltration dynamics in fractures with impermeable (top) and permeable (bottom) walls: (a) $Q=2 \times 10^{-8} \mathrm{~m}^{3} \mathrm{~s}^{-1}, t=4.560 \mathrm{~s}$; (b) $Q=$ $8 \times 10^{-8} \mathrm{~m}^{3} \mathrm{~s}^{-1}, t=0.912 \mathrm{~s}$; (c) $Q=6 \times 10^{-7} \mathrm{~m}^{3} \mathrm{~s}^{-1}, t=0.251 \mathrm{~s}$; (d) $Q=1 \times 10^{-6} \mathrm{~m}^{3} \mathrm{~s}^{-1}, t=0.228 \mathrm{~s} \ldots \ldots \ldots \ldots . \ldots . \ldots . \ldots 89$

6.12 Four scenarios of infiltration dynamics in fractures with impermeable and permeable walls: (1) for $Q<6 \times 10^{-8} \mathrm{~m}^{3} \mathrm{~s}^{-1}$ preferential flow and ponding occur simultaneously; (2) for $Q$ in the range between $6 \times 10^{-8}$ and $1 \times 10^{-7} \mathrm{~m}^{3} \mathrm{~s}^{-1}$ ponding is dominating; (3) for $Q$ in the range between $1 \times 10^{-7}$ and $8 \times 10^{-7} \mathrm{~m}^{3} \mathrm{~s}^{-1}$ preferential flow and ponding occur simultaneously; (4) for $Q<8 \times 10^{-7} \mathrm{~m}^{3} \mathrm{~s}^{-1}$ preferential flow is dominating. . . . . . . . . . . . . . . . . . 90

6.13 Flow mode distributions inside a rough fracture with impermeable (top) and permeable (bottom) walls for different fluxes at arrival times for permeable fracture: (a) $Q=3 \times 10^{-6} \mathrm{~m}^{3} \mathrm{~s}^{-1}, t=1.026 \mathrm{~s}$; (b) $Q=$ $9 \times 10^{-6} \mathrm{~m}^{3} \mathrm{~s}^{-1}, t=0.570 \mathrm{~s}$; (c) $Q=2 \times 10^{5} \mathrm{~m}^{3} \mathrm{~s}^{-1}, t=0.388 \mathrm{~s} \ldots 91$

6.14 Dependence of fluid arrival time on infiltration rate for an impermeable and permeable matrix. . . . . . . . . . . . . . . . . . 92

6.15 The outflow ratio over time for different infiltration rates: (a) $Q=$ $3 \times 10^{-6} \mathrm{~m}^{3} \mathrm{~s}^{-1}$; (b) $Q=9 \times 10^{-6} \mathrm{~m}^{3} \mathrm{~s}^{-1}$; (c) $Q=2 \times 10^{5} \mathrm{~m}^{3} \mathrm{~s}^{-1} \ldots .92$ 


\section{List of Tables}

4.1 Static contact angles of droplets for different solid-fluid interaction

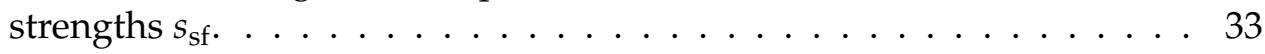

4.2 Effective static contact angles of droplets on rough hydrophobic and hydrophilic surfaces. fr: fine roughness; mr: medium roughness; cr: coarse roughness. . . . . . . . . . . . . . . . . . 4 40

4.3 Effective static contact angles of droplets with $R_{0}$ ranging from 0.5 to $1.6 \mathrm{~mm}$ on fine-, medium-, and coarse-roughness dual-rectangular hydrophobic surfaces. . . . . . . . . . . . . . . . . . 43

4.4 Effective static contact angles of droplets with $R_{\mathrm{eq}}=0.64 \mathrm{~mm}$ and $1.3 \mathrm{~mm}$ on a fine-roughness dual-rectangular hydrophobic surface depending on initial conditions. . . . . . . . . . . . . . . 46

5.1 Statistical properties for various fluxes in smooth and rough fractures. 63

5.2 Comparison of experimental (Nicholl and Glass; 2005, Fig. 27) and simulation data . . . . . . . . . . . . . . . . . . . 64

6.1 Standard deviations and standard errors of pressure heads at $t=1,6$, and 16 hours. . . . . . . . . . . . . . . . 83

6.2 Statistical properties for different fluxes in rough fractures. . . . . . . . 92 



\title{
List of Abbreviations
}

\author{
DFN Discrete Fracture Network \\ DPD Dissipative Particle Dynamics \\ EOS Equation Of State \\ LAMMPS Large-Scale Atomic/Molecular Massively Parallel Simulator \\ MD Molecular Dynamics \\ PF-SPH Pairwise-Force Smoothed Particle Hydrodynamics \\ REV Representative Elementary Volume \\ SDPD Smoothed Dissipative Particle Dynamics
}





\section{List of Symbols}

\begin{tabular}{|c|c|c|}
\hline$A_{\sim}$ & magnitude & $\mathrm{m}$ \\
\hline$\tilde{A}$ & interaction forces coefficient & - \\
\hline a & acceleration & $\mathrm{ms}^{-2}$ \\
\hline$b$ & fracture aperture & $\mathrm{m}$ \\
\hline$\tilde{B}$ & interaction forces coefficient & - \\
\hline Bo & Bond number & - \\
\hline$c$ & speed of sound & $\mathrm{ms}^{-1}$ \\
\hline $\mathrm{Ca}$ & capillary number & - \\
\hline$C_{m}$ & specific moisture capacity & $\mathrm{m}^{-1}$ \\
\hline$D$ & fractal dimension & - \\
\hline$d$ & distance & $\mathrm{m}$ \\
\hline$d_{m}$ & representative grain size & $\mathrm{m}$ \\
\hline$d_{\max }$ & upper grain size limit & $\mathrm{m}$ \\
\hline $\mathbf{F}$ & force & $\mathrm{N}$ \\
\hline$\Delta F_{g}$ & gravity force & $\mathrm{N}$ \\
\hline$\Delta F_{c}^{\circ}$ & capillary force & $\mathrm{N}$ \\
\hline$\Delta F_{v}$ & viscous force & $\mathrm{N}$ \\
\hline g & gravity & $\mathrm{ms}^{-2}$ \\
\hline H & height & $\mathrm{m}$ \\
\hline$h$ & kernel length & $\mathrm{m}$ \\
\hline$h_{g, v}$ & characteristic length scale & $\mathrm{m}$ \\
\hline$i^{\prime \prime \prime}$ & particle index & - \\
\hline$j$ & particle index & - \\
\hline$k$ & intrinsic fracture permeability & $\mathrm{m}^{2}$ \\
\hline$k_{r}$ & relative hydraulic conductivity & - \\
\hline $\mathbf{K}_{s}$ & saturated hydraulic conductivity & $\mathrm{ms}^{-1}$ \\
\hline$l$ & length & $\mathrm{m}$ \\
\hline$L_{t i p}$ & finger tip length & $\mathrm{m}$ \\
\hline $\bar{L}_{\text {tip }}$ & average length of stagnant finger & $\mathrm{m}$ \\
\hline$m$ & van Genuchten parameter & - \\
\hline$m_{0}$ & particle mass & $\mathrm{kg}$ \\
\hline$M\left(d<d_{m}\right)$ & percentage of mass less than $d_{m}$ & $\mathrm{~kg}$ \\
\hline$M_{\text {out }}$ & outflow mass & $\mathrm{kg}$ \\
\hline$M_{t}$ & total mass & $\mathrm{kg}$ \\
\hline$N$ & total number of particles in the domain & - \\
\hline$n$ & van Genuchten parameter & - \\
\hline $\mathbf{n}$ & normal vector & - \\
\hline$n_{d}$ & number density & - \\
\hline$n_{i}^{s}$ & phase-specific number density & $\mathrm{m}^{-3}$ \\
\hline$n_{i}$ & total particle density & $\mathrm{m}^{-3}$ \\
\hline$P$ & pressure & $\mathrm{Pa}$ \\
\hline$p_{c}$ & capillary pressure & $\mathrm{Pa}$ \\
\hline
\end{tabular}




\begin{tabular}{|c|c|c|}
\hline$q$ & specific flux & $\mathrm{m} \mathrm{s}^{-1}$ \\
\hline$Q$ & volumetric flux & $\mathrm{m} \mathrm{s}^{-3}$ \\
\hline$\widetilde{Q}_{\text {in }}$ & inflow volumetric flux & $\mathrm{m} \mathrm{s}^{-3}$ \\
\hline$Q_{\text {out }}$ & outflow volumetric flux & $\mathrm{m} \mathrm{s}^{-3}$ \\
\hline $\mathbf{r}$ & distance & $\mathrm{m}$ \\
\hline$r_{1}$ & first principal radius spanning both walls of the fracture & $\mathrm{m}$ \\
\hline$r_{2}$ & in-plane curvature & $\mathrm{m}$ \\
\hline$R_{0}$ & initial droplet radius & $\mathrm{m}$ \\
\hline $\operatorname{Re}$ & Reynolds number & \\
\hline$R_{\mathrm{eq}}$ & equilibrated droplet radius & $\mathrm{m}$ \\
\hline$r_{\max }$ & maximum pore radius & $\mathrm{m}$ \\
\hline$S$ & water-air-solid contact line & $\mathrm{m}^{-1}$ \\
\hline$S$ & standard deviation & - \\
\hline$s^{s}$ & standard velocity deviation in smooth fracture & - \\
\hline$s r$ & standard velocity deviation in rough fracture & - \\
\hline$S_{e}$ & effective saturation & - \\
\hline$s_{\mathrm{ff}}$ & fluid-fluid interaction coefficient & - \\
\hline$s_{\text {sf }}$ & solid-fluid interaction coefficient & - \\
\hline$S_{S}$ & specific storage coefficient & $\mathrm{Pa}^{-1}$ \\
\hline$S E$ & standard error & - \\
\hline$T$ & period & $\mathrm{m}$ \\
\hline$t$ & time & $\mathrm{s}$ \\
\hline$t^{*}$ & ratio between arrival times & - \\
\hline$t_{i m}$ & arrival time for impermeable fracture & $\mathrm{s}$ \\
\hline$t_{p}$ & arrival time for permeable fracture & s \\
\hline $\mathbf{v}$ & fluid velocity & $\mathrm{m} \mathrm{s}^{-1}$ \\
\hline$v$ & fingertip velocity & $\mathrm{m} \mathrm{s}^{-1}$ \\
\hline $\bar{v}$ & mean flow velocity & $\mathrm{ms}^{-1}$ \\
\hline$\hat{v}$ & scaled fingertip velocity & $\mathrm{m} \mathrm{s}^{-1}$ \\
\hline $\mathbf{v}_{b}$ & boundary velocity & $\mathrm{m} \mathrm{s}^{-1}$ \\
\hline$v_{s e c}^{i}$ & flow velocity of one fracture segment & $\mathrm{ms}^{-1}$ \\
\hline$v^{s, *}$ & normalized flow velocity in smooth fracture & $\mathrm{s}^{-1}$ \\
\hline$v_{\Delta=10,20}^{r, *}$ & normalized flow velocity in rough fracture & $\mathrm{s}^{-1}$ \\
\hline$v_{\mathrm{f}}^{*}$ & normalized velocity of individual fingers & $\mathrm{s}^{-1}$ \\
\hline$v_{s e c}^{\frac{1}{i}}$ & flow velocity of one segment & $\mathrm{m} \mathrm{s}^{-1}$ \\
\hline$\alpha$ & van Genuchten parameter & $\mathrm{m}^{-1}$ \\
\hline$\alpha_{k}$ & Wendland function coefficient & $\mathrm{m}^{-3}$ \\
\hline$\gamma$ & EOS coefficient & - \\
\hline$\Delta$ & Hurst exponent random variance & $\mathrm{m}$ \\
\hline$\Delta t$ & time step & $\mathrm{s}$ \\
\hline$\Delta x$ & particle spacing & $\mathrm{m}$ \\
\hline$\varepsilon_{0}$ & difference in static contact angles & $\circ$ \\
\hline$\varepsilon_{\text {eff }}$ & difference in effective contact angles & $\circ$ \\
\hline$\epsilon_{p}$ & porosity & - \\
\hline$\lambda$ & scaling ratio & - \\
\hline$\mu$ & viscosity & Pas \\
\hline$\eta$ & outflow ratio & - \\
\hline$\Delta \eta_{t=3}$ & difference in outflow ratios at $t=3 \mathrm{~s}$ & - \\
\hline$\phi$ & proximity ratio & - \\
\hline$\varphi$ & surface inclination angle & $\circ$ \\
\hline
\end{tabular}




$\begin{array}{lll}\psi & \text { hydraulic head } & \mathrm{m} \\ \psi_{b} & \text { hydraulic head of boundary particles } & \mathrm{m} \\ \psi_{f} & \text { hydraulic head of fluid particles } & \mathrm{m} \\ \psi_{d} & \text { draining pressure head } & \mathrm{m} \\ \psi_{w} & \text { wetting pressure head } & \mathrm{m} \\ \rho & \text { density } & \mathrm{kg} \mathrm{m}^{-3} \\ \sigma & \text { surface tension } & \mathrm{N} \mathrm{m}^{-1} \\ \tau_{w} & \text { viscous stress tensor } & \mathrm{Pa} \\ \Theta & \text { water content } & - \\ \Theta_{b} & \text { water content of boundary particles } & - \\ \Theta_{f} & \text { water content of fluid particles } & - \\ \Theta_{r} & \text { residual water content } & - \\ \Theta_{s} & \text { saturated water content } & - \\ \theta_{0} & \text { static contact angle } & \circ \\ \theta_{\mathrm{eff}} & \text { effective contact angle } & \circ \\ \chi_{f} & \text { fluid compressibility } & \mathrm{Pa}^{-1} \\ \chi_{p} & \text { porous matrix compressibility } & \mathrm{Pa}^{-1} \\ \zeta & \text { Hurst exponent } & - \\ \Omega_{f} & \text { domain with fluid particles } & - \\ \Omega_{s} & \text { domain with solid particles } & - \\ \Omega_{w a} & \text { water-air interface domain } & -\end{array}$





\section{Chapter 1}

\section{Introduction}

\subsection{Motivation}

The drinking water demand is increasing due to population growth, while the available drinking water resources are globally decreasing because of overexploitation and pollution (Hoekstra and Chapagain; 2011). Groundwater resources supply the largest amount of freshwater, and strongly depend on rainfall/infiltration events and recharge amounts (McWhorter et al.; 1977).

The infiltration dynamics involve all aspects of flow through a variably saturated porous medium, ranging in complexity from steady-state, saturated flow in a homogeneous, isotropic medium to transient, unstable, unsaturated flow through an anisotropic, heterogeneous medium (Bear; 2012). Understanding the nature of water movement in the vadose zone and its quantification is essential for solving a variety of problems, such as water resources management and vulnerability assessment (Council et al.; 1996), waste repositories (Evans and Rasmussen; 1991; Follin et al.; 2014), geothermal reservoir exploitation and heat storage (Fox et al.; 2013; Hofmann et al.; 2014), $\mathrm{CO}_{2}$ storage (Bond et al.; 2013), oil and gas recovery, fracking (Myers; 2012), and geotechnical applications (Berkowitz; 2002; Blaise Koffi et al.; 2013). All these applications require a deep understanding of the unsaturated flow processes, which are influenced by the subsurface properties, such as fracture/pore connectivity, fracture surface area and porous matrix/fracture interaction (Ebel and Nimmo; 2013; Flint et al.; 2001).

Fractured-porous media consist of porous permeable or impermeable matrix and fractures, which provide major conduits or barriers for fluid flows. Unsaturated or partially saturated flow through fractured-porous media is a complex process, which depends on geometrical fracture and porous-medium parameters (e.g., grain size distribution, structure, fracture thickness, fracture-wall geometry/roughness), physical (density, soil mineralogy, anisotropy) and hydraulic properties (wettability, permeability, saturation), see Fig. 1.1. Due to their complexity, flow processes though through the vadose zone are still poorly understood (Faybishenko et al.; 2015). Partially saturated hydrodynamics can be classified into two types: (1) diffuse/porousmedium flow, where water slowly homogeneously saturates the porous medium, and (2) preferential/fracture flow, where water follow path of less resistance and bypass parts of the pore structure (Nimmo; 2006). Both of these two flow types can also happen at the same time.

The mathematical description of diffuse flow relies on the Richards (1931) equation, which involves Brooks and Corey (1964) or Van Genuchten (1980) type of pressure-saturation relationships. Many models apply this approach not only to porousmedium flow, but also to porous-fractured media (Heilweil et al.; 2015; Kordilla et al.; 2012; Therrien and Sudicky; 1996). Such models have little or no resemblance to the original physical processes and/or they don't take into account important 


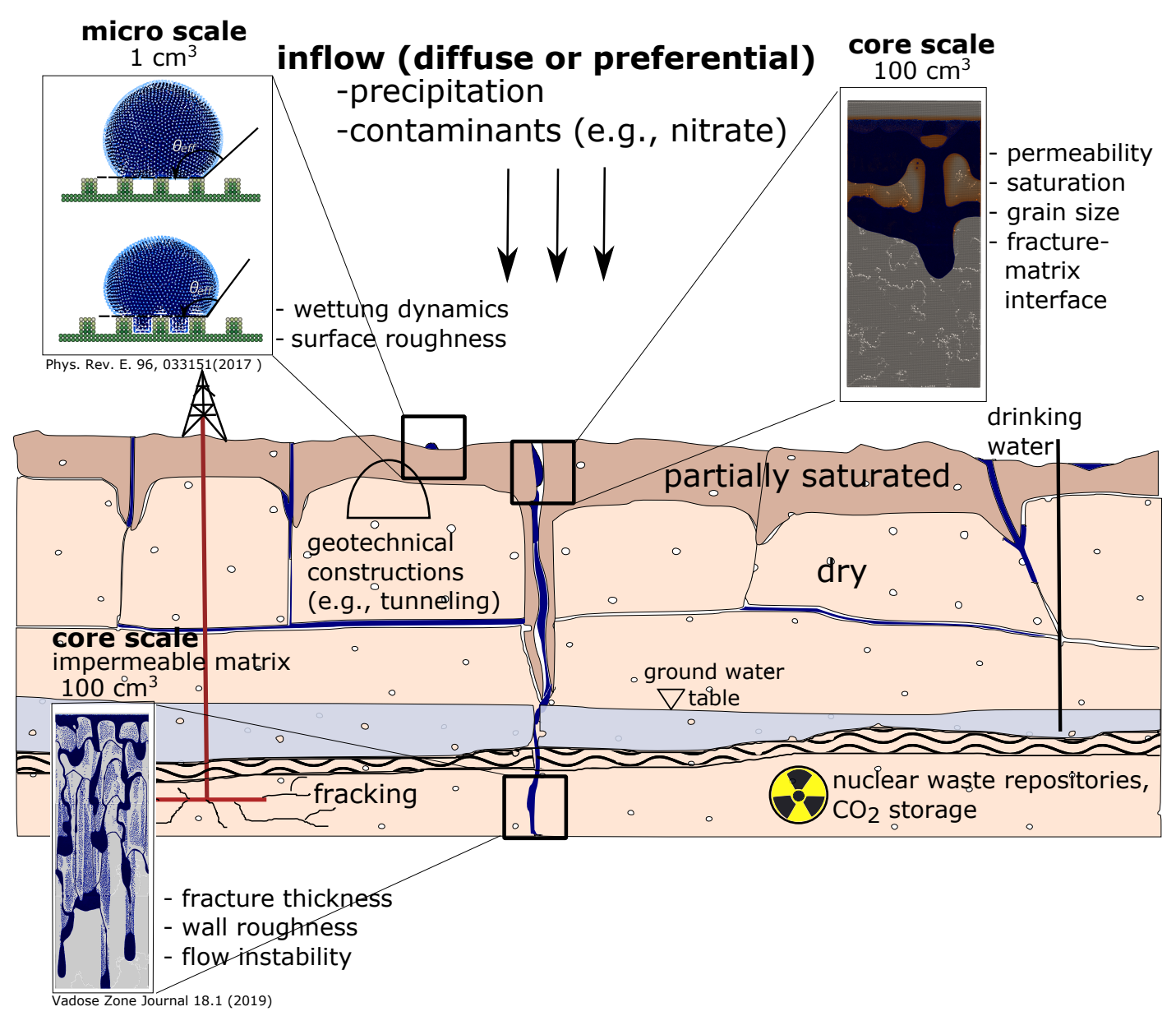

FIGURE 1.1: Geometrical and hydraulic subsurface properties influencing infiltration dynamics.

flow processes, e.g., fingering, preferential flow pathways (Nicholl and Glass; 2005), flow meandering, and erratic flow dynamics (Dragila and Weisbrod; 2004; Wood and Huang; 2015).

The mathematical description of fracture flow relies on the Navier-Stokes equation. In this case, flow through a single fracture can be approximated as a flow between two smooth parallel plates (Dragila and Weisbrod; 2003; Keller et al.; 1995). Many efforts have been made to demonstrate experimentally and numerically the influence of natural wall roughness on fracture flow (Poon et al.; 1992; Schmittbuhl et al.; 1995). These efforts can successfully reproduce flow instabilities in fractured media. However, they consider fracture walls being impermeable, and do not take into account fluid losses due to infiltration into the permeable fracture walls. Another challenge in characterizing flow in the unsaturated zone of fractured-porous media is the spatial scale contrast between pore-space and fracture void space, which makes it difficult to model flow processes with a single numerical approach.

In this work, both (diffuse and preferential flow) approaches are taken into account. This doctoral research is focused on the development of a numerical Smoothed Particle Hydrodynamics (SPH) model to study the infiltration dynamics in the vadose zone at core scales (Fig. 1.1), and to investigate the influence of geometrical (structure, wall roughness), and hydraulic (conductivity, wettability, saturation) parameters on preferential flow and rapid aquifer recharge. The first part of the thesis provides simulation results of sessile and transient droplets on hydrophobic and 
hydrophilic surfaces. The second part deals with infiltration instabilities in smooth and rough fractures. The third part considers flow through porous media, and at a fracture-matrix interface. The simulation results demonstrate the influence of the fracture wall permeability on arrival time and on the volume of water which can be stored in porous fracture walls.

\subsection{Objectives and approaches}

The results of this thesis are intended to provide a better understanding of infiltration dynamics in unsaturated fractured porous media, including the flow instability in smooth and rough fractures, and between matrix and fracture.

The main objectives this thesis are:

- Objective 1: Understand the effect of microscopic wetting dynamics due to (oriented) surface roughness on the macroscopic contact angles and flow behavior of droplets. Approach: SPH study of static and dynamic droplets on structured rough surfaces covering hydrophobic and hydrophilic conditions. The impact of the roughness orientation is considered via anisotropic fractal roughness of the solid surfaces.

- Objective 2: Identification of the connection between fracture roughness and flow modes on the onset of preferential flow dynamics, i.e. formation of instabilities in variably-saturated fractures. Approach: SPH modeling of infiltration dynamics under varying injection rates and fracture roughness as well as the identification of velocity distributions and dimensionless slug(droplet) flow characteristics under highly complex conditions.

- Objective 3: Determine the impact of fracture-matrix interaction during infiltration events on the formation of preferential flow dynamics and hence the partitioning into a diffuse (porous matrix) and rapid (fracture) domain. Approach: Development of a mechanistic multiscale SPH code to simulate gravity-driven free-surface and fracture flows coupled to a porous medium flow described by the Richards (1931) equation. The code enables the study of the wetting process caused by various rapid surface flow modes as well as the transport process across the matrix-fracture interface via an efficient exchange algorithm.

\subsection{Format of the thesis}

This doctoral thesis consists of 7 chapters, which contain the introduction, literature overview, model description, results of the main research, and conclusion.

Chapter 2 describes the complexity of flow in fractured-porous media, gives a brief introduction to the scale concept, and introduces various experimental, analytical and numerical methods to characterize fractured porous medium flow.

Chapter 3 gives an overview of the employed SPH code and its key elements, which were used, modified and implemented within this doctoral research. In particular, this includes the most important algorithms, governing SPH equations, and time stepping procedure. This chapter can be read in conjunction with the Chapters 4,5 , and 6 . 
Chapters 4 to 6 represent the main research work, which was done for this thesis. Chapters 4 and 5 have been published in peer-reviewed journals, and Chapter 6 has been submitted to a peer-reviewed journal.

Chapter 4 describes the effect of surface geometry/roughness and its orientation on contact angle and droplet flow, as well as, on hydrophobic or hydrophilic behavior of water droplets, which has an impact on flow velocity, and, as a result, on aquifer recharge.

Chapter 5 presents SPH simulations of unsaturated flow in smooth and rough fractures and explains the effects of fracture wall roughness on forming preferential flow pathways, flow mode distributions, and on average flow velocity.

Chapter 6 provides a coupled PF-SPH model for the Richards and Navier-Stockes equations to simulate flow and transport in fractured porous media. This chapter includes a model validation via comparison to a COMSOL model and small scale laboratory experiments. The simulation results demonstrate the effect of matrix imbibition on preferential flow in the fracture domain and at a fracture-matrix interface.

Chapter 7 gives a comprehensive conclusion with regard to the objectives of the thesis and provides suggestions for future research.

\section{Bibliography}

Bear, J. (2012). Hydraulics of groundwater, Courier Corporation.

Berkowitz, B. (2002). Characterizing flow and transport in fractured geological media: A review, Advances in Water Resources 25: 861-884.

Blaise Koffi, Y., Ahoussi, K. E., Kouadio, K. E., Kouassi, A. M., Kouassi, O., Kpangui, L. C. and Biemi, J. (2013). Study of the Vulnerability of Fractured Aquifers in the Context of an Implementation of a Modern Landfill: Application of Remote Sensing and the Geotechnical Method on the Site of Kossihouen (Côte d'Ivoire, West Africa), International Journal of Innovation and Applied Studies 2(4): 556-573.

Bond, C. E., Wightman, R. and Ringrose, P. S. (2013). The influence of fracture anisotropy on $\mathrm{CO}_{2}$ flow, Geophysical Research Letters 40(7): 1284-1289.

Brooks, R. and Corey, T. (1964). Hydraulic properties of porous media, Hydrology Papers, Colorado State University 24: 37.

Council, N. R. et al. (1996). Rock fractures and fluid flow: contemporary understanding and applications, National Academies Press.

Dragila, M. I. and Weisbrod, N. (2003). Parameters affecting maximum fluid transport in large aperture fractures, Advances in Water Resources 26(12): 1219-1228.

Dragila, M. I. and Weisbrod, N. (2004). Flow in menisci corners of capillary rivulets, Vadose Zone Journal 3(4): 1439-1442.

Ebel, B. A. and Nimmo, J. R. (2013). An alternative process model of preferential contaminant travel times in the unsaturated zone: Application to rainier mesa and shoshone mountain, nevada, Environmental Modeling $\mathcal{E}$ Assessment 18(3): 345-363.

Evans, D. D. and Rasmussen, T. C. (1991). Unsaturated flow and transport through fractured rock related to high-level waste repositories, Technical report, Nuclear Regulatory Commission. 
Faybishenko, B., Benson, S. M. and Gale, J. E. (2015). Fluid dynamics in complex fractured-porous systems, Vol. 210, John Wiley \& Sons.

Flint, A. L., Flint, L. E., Bodvarsson, G. S., Kwicklis, E. M. and Fabryka-Martin, J. (2001). Evolution of the conceptual model of unsaturated zone hydrology at Yucca Mountain, Nevada, Journal of Hydrology 247(1-2): 1-30.

Follin, S., Hartley, L., Rhén, I., Jackson, P., Joyce, S., Roberts, D. and Swift, B. (2014). A methodology to constrain the parameters of a hydrogeological discrete fracture network model for sparsely fractured crystalline rock, exemplified by data from the proposed high-level nuclear waste repository site at forsmark, sweden, Hydrogeology Journal 22(2): 313-331.

Fox, D. B., Sutter, D., Beckers, K. F., Lukawski, M. Z., Koch, D. L., Anderson, B. J. and Tester, J. W. (2013). Sustainable heat farming: modeling extraction and recovery in discretely fractured geothermal reservoirs, Geothermics 46: 42-54.

Heilweil, V. M., Benoit, J. and Healy, R. W. (2015). Variably saturated groundwater modelling for optimizing managed aquifer recharge using trench infiltration, Hydrological Processes 29(13): 3010-3019.

Hoekstra, A. Y. and Chapagain, A. K. (2011). Globalization of water: Sharing the planet's freshwater resources, John Wiley \& Sons.

Hofmann, H., Blöcher, G., Börsing, N., Maronde, N., Pastrik, N. and Zimmermann, G. (2014). Potential for enhanced geothermal systems in low permeability limestones-stimulation strategies for the western malm karst (bavaria), Geothermics 51: 351-367.

Keller, A. A., Roberts, P. V. and Kitanidis, P. K. (1995). Prediction of single phase transport parameters in a variable aperture fracture, Geophysical Research Letters 22(11): 1425-1428.

Kordilla, J., Sauter, M., Reimann, T. and Geyer, T. (2012). Simulation of saturated and unsaturated flow in karst systems at catchment scale using a double continuum approach, Hydrology and Earth System Sciences 16(10): 3909-3923.

McWhorter, D. B., Sunada, D. K. and Sunada, D. K. (1977). Ground-water hydrology and hydraulics, Water Resources Publication.

Myers, T. (2012). Potential contaminant pathways from hydraulically fractured shale to aquifers, Groundwater 50(6): 872-882.

Nicholl, M. J. and Glass, R. J. (2005). Infiltration into an analog fracture: Experimental observations of gravity-driven fingering, Vadose Zone Journal 4: 1123-1151.

Nimmo, J. R. (2006). Unsaturated zone flow processes, Encyclopedia of hydrological sciences.

Poon, C., Sayles, R. and Jones, T. (1992). Surface measurement and fractal characterization of naturally fractured rocks, Journal of Physics D: Applied Physics 25(8): 1269.

Richards, L. A. (1931). Capillary conduction of liquids through porous mediums, Physics 1(5): 318-333.

Schmittbuhl, J., Schmitt, F. and Scholz, C. (1995). Scaling invariance of crack surfaces, Journal of Geophysical Research: Solid Earth 100(B4): 5953-5973. 
Therrien, R. and Sudicky, E. (1996). Three-dimensional analysis of variably-saturated flow and solute transport in discretely-fractured porous media, Journal of Contaminant Hydrology 23(1-2): 1-44.

Van Genuchten, M. T. (1980). A closed-form equation for predicting the hydraulic conductivity of unsaturated soils 1, Soil Science Society of America Journal 44(5): 892898.

Wood, T. and Huang, H. (2015). Experimental and modeling studies of episodic airwater two-phase flow in fractures and fracture networks, Fluid dynamics in complex fracturedporous systems. Wiley, Chichester, UK pp. 209-228. 


\section{Chapter 2}

\section{General overview}

\subsection{Fractured porous media}

Most consolidated porous rocks have undergone metamorphic processes or tectonically induced stress fields, which lead to discontinuities within the porous matrix (Nelson; 2001). Naturally fractured porous media consist of pore networks and interconnected fractures. Large pores and crevices are associated with fractures and have dimensions of $1 \times 10^{-4} \mathrm{~m}$ to $1 \times 10^{-2} \mathrm{~m}$ (Fischer et al.; 1998; Tsang and Tsang; 1987), while pore throats of the matrix have dimensions of $1 \times 10^{-7} \mathrm{~m}$ to $1 \times 10^{-5} \mathrm{~m}$ (Thoma et al.; 1992). However, the overall porosity of fractured-porous rocks is still dominated by the porous matrix (Singhal and Gupta; 2010). Nevertheless, fractures are considered fast transport pathways (Zimmerman and Bodvarsson; 1996), but under partial saturation conditions they can also impede flow (Wang and Narasimhan; 1985). Individual fractures can be characterized by their location, orientation, length, aperture, and roughness (Fossen; 2016), while fracture networks require also the characterization of fracture density and connectivity (Berkowitz; 2002).

\subsection{Characterization of flow in unsaturated fractured porous media}

The complexity of flow in fractured porous media is coarsed by the scale concept, where the fracture thickness is associated with a small scale, while the fracture length can extend over a large scale. The term "scale" describes a dimensional spectrum of the system, e.g., characteristic length and time (Faybishenko et al.; 2003, Fig. 2.1). The scale concept is based on the assumption that components of the system behave differently on different scales. The large scale allows to study flow in fracture networks and regional hydrogeological processes. Intermediate scales can be used to describe volume-averaged flow in fracture networks and fracture-matrix interactions. The small scale allows to describe in detail flow processes in one or few intersecting fractures. The core scale allows specific experiments for detailed investigations of flow in individual fractures and the adjacent porous matrix.

The strong contrasts in spatial scales between the fracture aperture and the extension of the fracture length and the scale contrast in the pores of the matrix make the characterization of infiltration dynamics in fractured porous media difficult with a single approach. There are several methodological approaches to describe infiltration dynamics in fractured-porous media in the unsaturated zone, which can be divided into three categories: experimental, theoretical and numerical. 


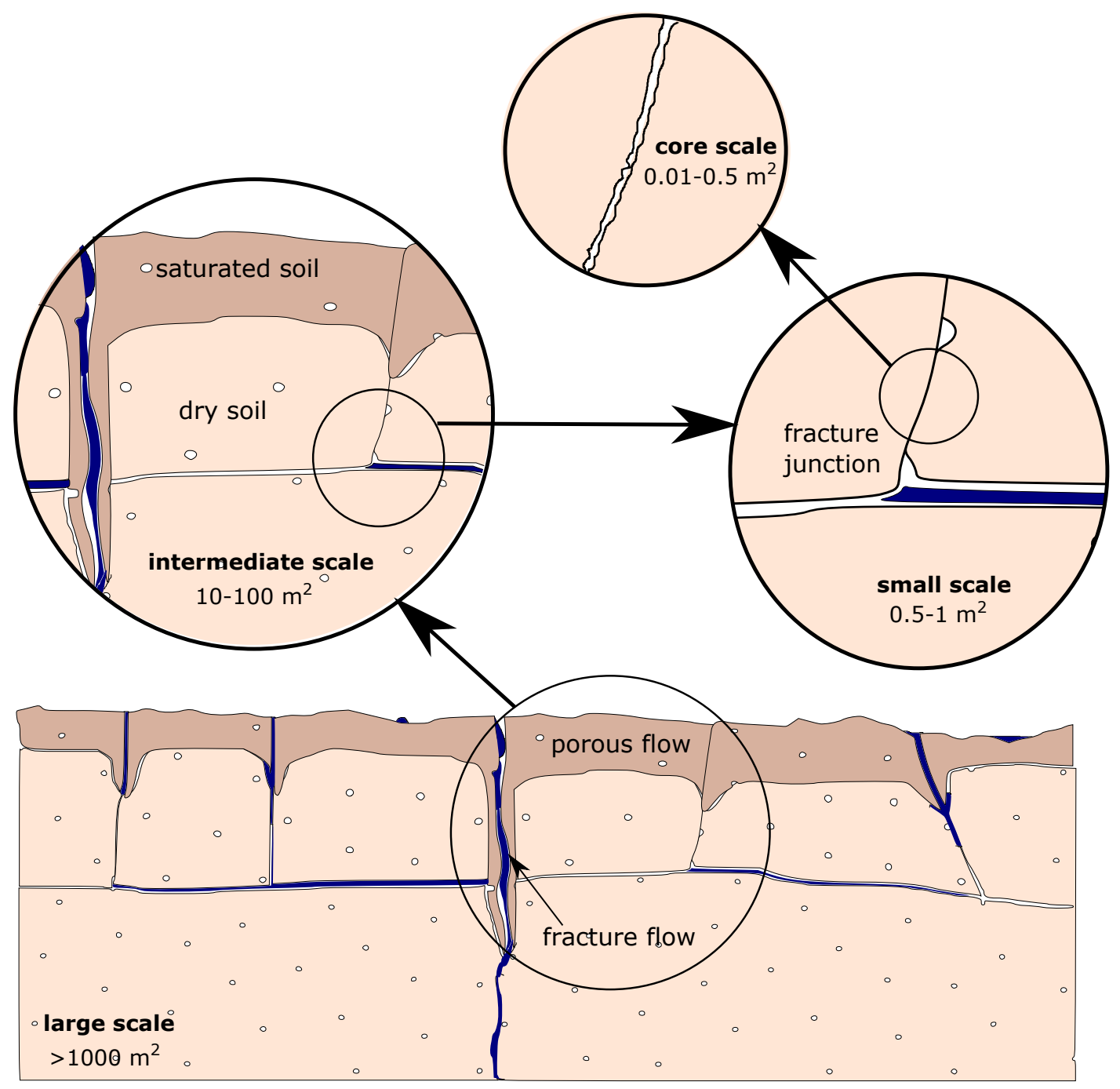

FIGURE 2.1: Scale hierarchy of fractured rocks in the unsaturated zone. Modified from Faybishenko et al. (2003)

\subsubsection{Experimental approaches}

Most of the laboratory experiments characterizing flow in fractures are limited to small scale and simple configuration setups. A common experimental setup in this case consists of two parallel (textured) glass plates (Hele-Shaw cell) with a small aperture, representing a fracture (Dragila and Weisbrod; 2003; Jones et al.; 2018; Nicholl et al.; 1994; Nicholl and Glass; 2005; Su et al.; 2001). These experimental setups successfully demonstrate fingering effects, and the dependence of flow velocity on infiltration rate, as well as free surface flow (Benson; 2001; Tokunaga and Wan; 1997; Tokunaga et al.; 2000). Tokunaga and Wan (1997), for example, investigated the effects of capillary pressure on free-surface film flows, which shows the importance of the porous matrix system on the effectiveness of rapid fracture discharge. Kordilla et al. (2017) showed experimentally that flow modes (droplet, rivulet, or transition between them) heavily influence the partioning behavior of water flowing along a fracture junction. Some laboratory studies take natural rough fracture surfaces into account to observe water infiltration in initially dry fractures (Brown et al.; 1998; Tokunaga and Wan; 2001). Brown et al. (1998) found that fracture channeling caused by fracture roughness can lead to breakthrough velocities or exceed the mean flow 
velocity.

Field investigations of unsaturated flow often deal with preferential flow pathways, - the phenomenon of irregular soil profile wetting, and, as a result, some parts of the soil with fast moving water (Hendrickx and Flury; 2001). Several authors (Berkowitz; 2002; Nimmo; 2012) have reviewed the observations and investigations of preferential flow under unsaturated conditions during field experiments. Simple field experimental setups are drip detectors and collection vessels installed below a fractured unsaturated rock mass, which allow to collect hydrogeological data, such as discharge, temperature, electrical conductivity, and turbidity in the unsaturated zone (Faybishenko et al.; 2000; Pronk et al.; 2009; Wood and Huang; 2015). These findings are useful for quantification of water storage and contaminant transport. However, such datasets commonly describe the hydraulic response of the bulk system and are not able to characterize fractures and associated properties in most cases. Thus, one of the fundamental problems is the determination of a fracture location, thickness, and orientation, as well as the formation of preferential flow paths based on measured data (Berkowitz; 2002).

\subsubsection{Theoretical approaches}

Theoretical approaches are based on an analytical solutions of flow equations, and can be used to validate numerical models. Analytical solutions can be subdivided into volume-effective (e.g., Richards equation) and discrete solutions, e.g. timedependent free-surface flow.

Volume-effective solutions often neglect the effects of preferential flow paths and are commonly applied for large-scale characterization. Most of volume-effective approaches are based on dual-domain concepts (Germann et al.; 2007; Nimmo; 2010), which assume that the porous medium consists of two interacting regions, one of them is associated with the fracture system, another one with the rock matrix. Dualdomain models can have a dual-porosity (Van Genuchten and Wierenga; 1976) or dual-permeability (Gerke and Köhne; 2004; Lassabatere et al.; 2014) description of the flow processes. The main difference between them is that dual-porosity models assume no water flow in the rock matrix, while dual-permeability models allow water flow in the rock matrix as well (Šimunek et al.; 2003). The study of Nimmo (2010) presents a dual-domain model, where the diffuse-flow domain (rock matrix) is always active, while the source-responsive domain (micropores or fractures) is only sometimes active, depending on the water source, i.e. rainfall event, which gives a better prediction of the height of water table at different times.

Discrete solutions take into consideration a single specific flow mode (droplet, rivulet, film). The main flow regimes are adsorbed film flow with average flow velocities of $3 \times 10^{-7} \mathrm{~ms}^{-1}$, droplet flow, rivulet flow if flow rates are high enough to prevent a breakup into droplets, and, at even higher flow rates free-surface films may develop (Ghezzehei; 2004). Tokunaga and Wan (1997) demonstrated that adsorbed films are an important mechanism for unsaturated flow in fractures, and a fast flow process in contrast to diffuse flow in the porous matrix.

Numerous studies have been carried out to characterize the droplet flow regime, including solutions for stationary shape (Gomba and Homsy; 2009), maximum height (De Gennes et al.; 2013), and contact angle (Yuan and Lee; 2013) of sessile droplets. Depending on wetting dynamics (hydrophobic or hydrophilic type of surfaces) and on surface geometry, contact angles of sessile droplets can vary over large ranges. 
On a rough surface, droplets can be in a Cassie state (Cassie and Baxter; 1944) if they touch only exposed parts of the rough surface, or in a Wenzel state (Wenzel; 1936) if they fill all depressions of the surface. Transient dynamics of droplets including breakup mechanisms have been investigated by Voinov (1976), Ghezzehei and Or (2005), and Lunati and Or (2009). A general linear relationship between Bond and Capillary number for a sliding droplet was found by Podgorski et al. (2001).

At a certain flow rate rivulets start to form. Rivulets are the main flow modes that persist during the preferential flow (Germann et al.; 2007). Ghezzehei (2004) derived an analytical solution for total flow rate of rivulets on smooth inclined surfaces. Dragila and Weisbrod (2003) and Dragila and Weisbrod (2004) provide an analytical solution for rivulet flow between two parallel plates. The recent study of McCreery et al. (2005) provides an analytical prediction of rivulet velocity and Reynolds number based on hydraulic diameter of the rivulet for any given aperture spacing. This study demonstrates, as well, a linear dependence of rivulet width on the flow rate.

At extremely high flow rates falling films start to form. Mikielewicz and Moszynskl (1976) provide an analytical solution for minimum thickness of a liquid film flowing on a vertical solid surface. Ghezzehei (2004) derives expressions for the critical flow rate at which a stable film breaks into rivulets. A steady state film flow with low Reynolds numbers is described by the laminar film theory of Nusselt (1916). However, for high Reynolds numbers $(R e>20)$ film flow may contain ripples and waves, which travel at much higher velocities and provide rapid fluid infiltration (Dragila and Wheatcraft; 2001).

\subsubsection{Numerical approaches}

Several classifications of numerical models exist. They can, for example, be based on the specification of the flow field (Eulerian and Lagrangian) (Batchelor and Batchelor; 2000), spatial discretization (grid-based or meshless models), spatial dimensions $(1,2$, or 3D), scale concept (micro, intermediate, or large scale), geometrical properties (e.g., single fracture or fracture network), or number of phases and components (single-, two-, multi-phase). Here, we give some examples of numerical models and their classifications which are used for characterizing the flow in fractured porous media.

In hydrogeology, both Eulerian and Lagrangian approaches are used to model fluid flow (Huyakorn; 2012), where the Eulerian grid is used for recording the properties of the fluid elements passing through a particular point or volume in space and Lagrangian markers are used to visualize their motion in space and time (Batchelor and Batchelor; 2000).

Figure 2.2 demonstrates the scale hierarchy of numerical techniques, which are commonly used for solving hydrogeological problems. The grid-based models are suitable for large spatial and temporal scales, while meshless models are used for detailed investigations of fluid motion at small or even micro scale.

Grid-based methods are often based on the Eulerian approach. Common numerical grid-based methods include finite element, finite difference, and finite volume methods, which are used to approximate the solution of governing differential equations by dissecting the domain into meshes, and applying simpler equations to individual elements or nodes in the mesh (Ismail-Zadeh and Tackley; 2010).

For fracture flow the grid based models can be subdivided into (Berre et al.; 2018):

- Single-continuum models, where fractures are represented by adapting the permeability of the porous medium. They require a representative elementary 


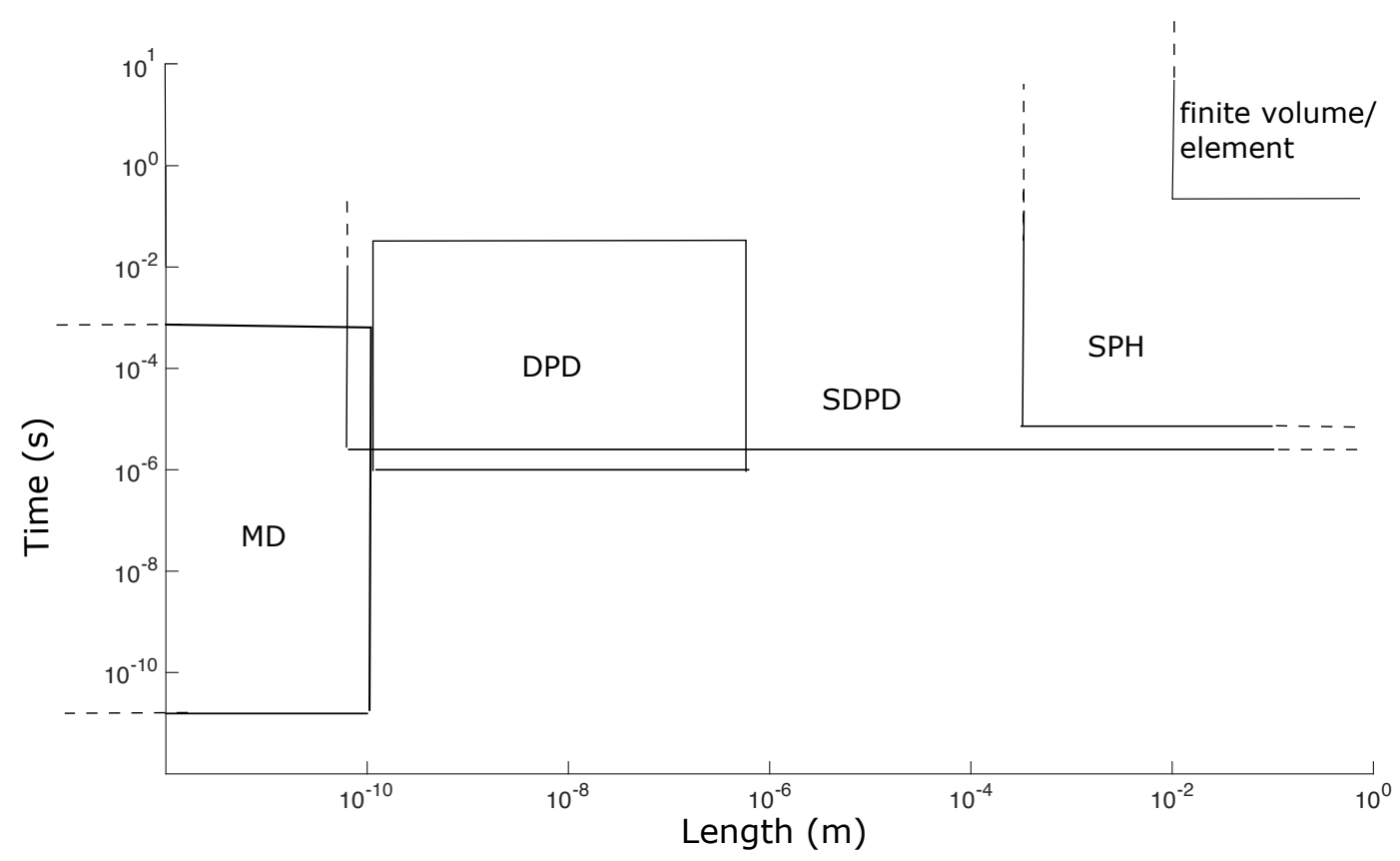

FIGURE 2.2: Scale hierarchy of numerical techniques used in hydrogeology. MD: molecular dynamics; DPD: dissipative particle dynamics; SDPD: smoothed dissipative particle dynamics; SPH: moothed particle hydrodynamics. Information compiled from Dzwinel et al. (1999, 2006); Kulkarni et al. (2013)

volume (REV) (Bear and Braester; 1972) to be defined and are often modeled with the Richards (1931) equation. Originally, this approach has been derived for porous media, but with some approximations can be applied to fractured media as well. However, depending on the type of heterogeneity they may not be able to capture effects of preferential flow.

- Multi-continuum approaches represent the fractured porous medium by several superimposed media with their own conservation equations. As in case of single-continuum models, they rely on the REV concept.

- Discrete fracture network (DFN) models can model flow focusing and the combined effect of diffuse matrix and rapid fracture flow (Hyman et al.; 2015; Mak; 2014; Zhang et al.; 2004).

Meshless methods are commonly based on Lagrangian approaches, which do not require a connection between nodes of the simulation domain, but are rather based on interaction of each node with all its neighbors. In hydrology such methods are commonly applied to study small-scale flow and transport physics. The most common meshless methods are the following:

- Smoothed particle hydrodynamics (SPH) models (Gingold and Monaghan; 1977; Lucy; 1977) commonly discretize the Navier-Stokes equation, where particle accelerations are computed from a pressure gradient (Kordilla et al.; 2017; Kordilla J.; 2013; Meakin and Tartakovsky; 2009; Shigorina et al.; 2017, 2019; Tartakovsky and Panchenko; 2016; Tartakovsky and Meakin; 2005).

- Dissipative particle dynamics (DPD) models (Hoogerbrugge and Koelman; 1992; Koelman and Hoogerbrugge; 1993) are used for simulating the dynamic 
and rheological properties of simple and complex fluids, where particle represents a cluster of atoms or molecules (Bian et al.; 2012; Huang and Meakin; 2008).

- Smoothed dissipative particle dynamics (SDPD) is a mesoscale particle-based model. The governing equations show close resemblance with the DPD model. SDPD has been used to study the dynamics of complex fluids such as particle suspensions and dilute DNA solutions (Bian et al.; 2012; Litvinov et al.; 2011).

- Molecular dynamics (MD) models (Alder and Wainwright; 1959) allow the closest approximation of the atomistic properties of a system. They have been employed to study capillary flow dynamics (Wei et al.; 2014), contact line dynamics (Wang and Zhao; 2013), and biological flow phenomena (Fedosov et al.; 2014).

The research work of this thesis is carried out with a SPH model. In comparison with grid-based and other meshless methods, SPH has several advantages:

- The main advantage of SPH method in comparison to other meshless methods is that SPH allows to simulate flow on a larger spatial and temporal scale (Fig. 2.2), than other particle techniques.

- The SPH method allows to investigate infiltration dynamics based on wetting conditions (e.g., hydrophobic or hydrophilic surfaces), and natural fracture roughness can be easily implemented, which can be difficult with grid-based methods.

- The SPH method is mass conservative, and no additional discretization of the mass-conservation equation is required.

- The SPH model allows to simulate transient flow dynamics over a wide range of wetting conditions without any need of resolving the air-phase, which provides a computational advantage, especially under low saturation conditions, i.e. large amount of continuous air-phase.

\section{Bibliography}

Alder, B. J. and Wainwright, T. E. (1959). Studies in molecular dynamics. i. general method, The Journal of Chemical Physics 31(2): 459-466.

Batchelor, C. K. and Batchelor, G. (2000). An introduction to fluid dynamics, Cambridge University Press.

Bear, J. and Braester, C. (1972). On the flow of two immscible fluids in fractured porous media, Developments in Soil Science, Vol. 2, Elsevier, pp. 177-202.

Benson, D. A. (2001). A model of water streaking down a wall, Water Resources Research 37(2): 427-430.

Berkowitz, B. (2002). Characterizing flow and transport in fractured geological media: A review, Advances in Water Resources 25: 861-884.

Berre, I., Doster, F. and Keilegavlen, E. (2018). Flow in fractured porous media: A review of conceptual models and discretization approaches, Transport in Porous Media pp. 1-22. 
Bian, X., Litvinov, S., Qian, R., Ellero, M. and Adams, N. A. (2012). Multiscale modeling of particle in suspension with smoothed dissipative particle dynamics, Physics of Fluids 24(1): 012002.

Brown, S., Caprihan, A. and Hardy, R. (1998). Experimental observation of fluid flow channels in a single fracture, Journal of Geophysical Research: Solid Earth 103(B3): 5125-5132.

Cassie, A. and Baxter, S. (1944). Wettability of porous surfaces, Transactions of the Faraday Society 40: 546-551.

De Gennes, P.-G., Brochard-Wyart, F. and Quéré, D. (2013). Capillarity and wetting phenomena: drops, bubbles, pearls, waves, Springer Science \& Business Media.

Dragila, M. I. and Weisbrod, N. (2003). Parameters affecting maximum fluid transport in large aperture fractures, Advances in Water Resources 26(12): 1219-1228.

Dragila, M. I. and Weisbrod, N. (2004). Flow in menisci corners of capillary rivulets, Vadose Zone Journal 3(4): 1439-1442.

Dragila, M. I. and Wheatcraft, S. W. (2001). Free-surface films, Conceptual models of flow and transport in the fractured vadose zone pp. 217-241.

Dzwinel, W., Alda, W. and Yuen, D. A. (1999). Cross-scale numerical simulations using discrete particle models, Molecular Simulation 22(6): 397-418.

Dzwinel, W., Yuen, D. A. and Boryczko, K. (2006). Bridging diverse physical scales with the discrete-particle paradigm in modeling colloidal dynamics with mesoscopic features, Chemical Engineering Science 61(7): 2169-2185.

Faybishenko, B., Bodvarsson, G. and Salve, R. (2003). On the physics of unstable infiltration, seepage, and gravity drainage in partially saturated tuffs, Journal of Contaminant Hydrology 62: 63-87.

Faybishenko, B., Witherspoon, P. A. and Benson, S. M. (2000). Dynamics of fluids in fractured rock, Washington DC American Geophysical Union Geophysical Monograph Series 122.

Fedosov, D. A., Noguchi, H. and Gompper, G. (2014). Multiscale modeling of blood flow: from single cells to blood rheology, Biomechanics and modeling in mechanobiology 13(2): 239-258.

Fischer, U., Kulli, B. and Flühler, H. (1998). Constitutive relationships and pore structure of undisturbed fracture zone samples with cohesionless fault gouge layers, Water Resources Research 34(7): 1695-1701.

Fossen, H. (2016). Structural geology, Cambridge University Press.

Gerke, H. H. and Köhne, J. M. (2004). Dual-permeability modeling of preferential bromide leaching from a tile-drained glacial till agricultural field, Journal of Hydrology 289(1-4): 239-257.

Germann, P., Helbling, A. and Vadilonga, T. (2007). Rivulet approach to rates of preferential infiltration, Vadose Zone Journal 6(2): 207-220.

Ghezzehei, T. (2004). Constraints for flow regimes on smooth fracture surfaces, Water Resources Research 40(11). 
Ghezzehei, T. A. and Or, D. (2005). Liquid fragmentation and intermittent flow regimes in unsaturated fractured media, Water resources research 41(12).

Gingold, R. A. and Monaghan, J. J. (1977). Smoothed particle hydrodynamics: theory and application to non-spherical stars, Monthly notices of the royal astronomical society 181(3): 375-389.

Gomba, J. M. and Homsy, G. M. (2009). Analytical solutions for partially wetting two-dimensional droplets, Langmuir 25(10): 5684-5691.

Hendrickx, J. M. and Flury, M. (2001). Uniform and preferential flow mechanisms in the vadose zone, Conceptual models of flow and transport in the fractured vadose zone pp. 149-187.

Hoogerbrugge, P. and Koelman, J. (1992). Simulating microscopic hydrodynamic phenomena with dissipative particle dynamics, EPL (Europhysics Letters) 19(3): 155.

Huang, H. and Meakin, P. (2008). Three-dimensional simulation of liquid drop dynamics within unsaturated vertical hele-shaw cells, Water Resources Research 44(3).

Huyakorn, P. S. (2012). Computational methods in subsurface flow, Academic Press.

Hyman, J. D., Karra, S., Makedonska, N., Gable, C. W., Painter, S. L. and Viswanathan, H. S. (2015). dfnworks: A discrete fracture network framework for modeling subsurface flow and transport, Computers \& Geosciences 84: 10-19.

Ismail-Zadeh, A. and Tackley, P. (2010). Computational methods for geodynamics, Cambridge University Press.

Jones, B. R., Brouwers, L. B. and Dippenaar, M. A. (2018). Partially to fully saturated flow through smooth, clean, open fractures: qualitative experimental studies, $\mathrm{Hy}$ drogeology Journal 26(3): 945-961.

Koelman, J. and Hoogerbrugge, P. (1993). Dynamic simulations of hard-sphere suspensions under steady shear, EPL (Europhysics Letters) 21(3): 363.

Kordilla, J., Noffz, T., Dentz, M., Geyer, T. and Tartakovsky, A. M. (2017). Effect of unsaturated flow modes on partitioning dynamics of gravity-driven flow at a simple fracture intersection: laboratory study and three-dimensional smoothed particle hydrodynamics simulations., Water Resources Research 53(11): 9496-9518.

Kordilla J., Tartakovsky A.M., G. T. (2013). A smoothed particle hydrodynamics model for droplet and film flow on smooth and rough fracture surfaces, Advances in Water Resources 59: 1-14.

Kulkarni, P. M., Fu, C.-C., Shell, M. S. and Gary Leal, L. (2013). Multiscale modeling with smoothed dissipative particle dynamics, The Journal of Chemical Physics 138(23): 234105.

Lassabatere, L., Yilmaz, D., Peyrard, X., Peyneau, P. E., Lenoir, T., Šimunek, J. and Angulo-Jaramillo, R. (2014). New analytical model for cumulative infiltration into dual-permeability soils, Vadose Zone Journal 13(12).

Litvinov, S., Hu, X. and Adams, N. (2011). Numerical simulation of tethered dna in shear flow, Journal of Physics: Condensed Matter 23(18): 184118. 
Lucy, L. B. (1977). A numerical approach to the testing of the fission hypothesis, The Astronomical Journal 82: 1013-1024.

Lunati, I. and Or, D. (2009). Gravity-driven slug motion in capillary tubes, Physics of fluids 21(5): 052003.

Mak, S. W. (2014). Assessing fracture network connectivity of prefeasibility-level high temperature geothermal projects using discrete fracture network modelling, $\mathrm{PhD}$ thesis, University of British Columbia.

McCreery, G., Meakin, P., McEligot, D. et al. (2005). Rivulet flow in vertical parallelwall channels, Technical report, Tech. report INL/EXT-05-00489, Idaho National Laboratory.

Meakin, P. and Tartakovsky, A. M. (2009). Modeling and simulation of pore-scale multiphase fluid flow and reactive transport in fractured and porous media, $R e-$ views of Geophysics 47(3).

Mikielewicz, J. and Moszynskl, J. (1976). Minimum thickness of a liquid film flowing vertically down a solid surface, International Journal of Heat and Mass Transfer 19(7): 771-776.

Nelson, R. (2001). Geologic analysis of naturally fractured reservoirs, Elsevier.

Nicholl, M., Glass, R. and Wheatcraft, S. (1994). Gravity-driven infiltration instability in initially dry nonhorizontal fractures, Water Resources Research 30(9): 2533-2546.

Nicholl, M. J. and Glass, R. J. (2005). Infiltration into an analog fracture: Experimental observations of gravity-driven fingering, Vadose Zone Journal 4: 1123-1151.

Nimmo, J. R. (2010). Theory for source-responsive and free-surface film modeling of unsaturated flow, Vadose Zone Journal 9: 295-306.

Nimmo, J. R. (2012). Preferential flow occurs in unsaturated conditions, Hydrological Processes 26(5): 786-789.

Nusselt, W. (1916). Die Oberflächenkondensation des Wasserdampfes, vdizeitschrift 60: 542-575.

Podgorski, T., Flesselles, J.-M. and Limat, L. (2001). Corners, cusps, and pearls in running drops, Physical Review Letters 87(3): 036102.

Pronk, M., Goldscheider, N., Zopfi, J. and Zwahlen, F. (2009). Percolation and particle transport in the unsaturated zone of a karst aquifer, Groundwater 47(3): 361-369.

Richards, L. A. (1931). Capillary conduction of liquids through porous mediums, Physics 1(5): 318-333.

Shigorina, E., Kordilla, J. and Tartakovsky, A. M. (2017). Smoothed particle hydrodynamics study of the roughness effect on contact angle and droplet flow, Physical Review E 96(3): 033115.

Shigorina, E., Tartakovsky, A. M. and Kordilla, J. (2019). Investigation of gravitydriven infiltration instabilities in smooth and rough fractures using a pairwiseforce smoothed particle hydrodynamics model, Vadose Zone Journal 18(1). 
Singhal, B. B. S. and Gupta, R. P. (2010). Applied hydrogeology of fractured rocks, Springer Science \& Business Media.

Su, G. W., Geller, J. T., Pruess, K. and Hunt, J. R. (2001). Solute transport along preferential flow paths in unsaturated fractures, Water Resources Research 37(10): 24812491.

Tartakovsky, A. M. and Panchenko, A. (2016). Pairwise force smoothed particle hydrodynamics model for multiphase flow: surface tension and contact line dynamics, Journal of Computational Physics 305: 1119-1146.

Tartakovsky, A. and Meakin, P. (2005). Modeling of surface tension and contact angles with smoothed particle hydrodynamics, Physical Review E 72(2).

Thoma, S. G., Gallegos, D. P. and Smith, D. M. (1992). Impact of fracture coatings on fracture/matrix flow interactions in unsaturated, porous media, Water Resources Research 28(5): 1357-1367.

Tokunaga, T. K. and Wan, J. (1997). Water film flow along fracture surfaces of porous rock, Water Resources Research 33.

Tokunaga, T. K. and Wan, J. (2001). Surface-zone flow along unsaturated rock fractures, Water Resources Research 37(2): 287-296.

Tokunaga, T. K., Wan, J. and Sutton, S. R. (2000). Transient film flow on rough fracture surfaces, Water Resources Research 36(7): 1737-1746.

Tsang, Y. W. and Tsang, C. (1987). Channel model of flow through fractured media, Water Resources Research 23(3): 467-479.

Van Genuchten, M. T. and Wierenga, P. (1976). Mass transfer studies in sorbing porous media i. analytical solutions 1, Soil Science Society of America Journal 40(4): 473-480.

Voinov, O. (1976). Hydrodynamics of wetting, Fluid Dynamics 11(5): 714-721.

Šimunek, J., Jarvis, N. J., Van Genuchten, M. T. and Gärdenäs, A. (2003). Review and comparison of models for describing non-equilibrium and preferential flow and transport in the vadose zone, Journal of Hydrology 272(1-4): 14-35.

Wang, F.-C. and Zhao, Y.-P. (2013). Contact angle hysteresis at the nanoscale: a molecular dynamics simulation study, Colloid and Polymer Science 291(2): 307-315.

Wang, J. and Narasimhan, T. N. (1985). Hydrologic mechanisms governing fluid flow in a partially saturated, fractured, porous medium, Water Resources Research 21(12): 1861-1874.

Wei, N., Lv, C. and Xu, Z. (2014). Wetting of graphene oxide: A molecular dynamics study, Langmuir 30(12): 3572-3578.

Wenzel, R. N. (1936). Resistance of solid surfaces to wetting by water, Industrial $\mathcal{E}$ Engineering Chemistry 28(8): 988-994.

Wood, T. and Huang, H. (2015). Experimental and modeling studies of episodic airwater two-phase flow in fractures and fracture networks, Fluid dynamics in complex fracturedporous systems. Wiley, Chichester, UK pp. 209-228. 
Yuan, Y. and Lee, T. R. (2013). Contact angle and wetting properties, Surface Science Techniques, Springer, pp. 3-34.

Zhang, K., Wu, Y.-S., Bodvarsson, G. and Liu, H.-H. (2004). Flow focusing in unsaturated fracture networksa numerical investigation, Vadose Zone Journal 3(2): 624633.

Zimmerman, R. W. and Bodvarsson, G. S. (1996). Hydraulic conductivity of rock fractures, Transport in Porous Media 23(1): 1-30. 



\section{Chapter 3}

\section{Multiscale pairwise force Smoothed Particle Hydrodynamics model}

The intention of this chapter is to give an overview of the SPH method and the simulation algorithm used in the studies described in Chapters 4, 5, and 6 .

\subsection{PF-SPH method and governing equations}

SPH is a mesh-free Lagrangian method where fluids are discretized with a set of $N$ points, commonly referred to as particles. Each particle is defined by its position $\mathbf{r}_{i}$, mass $m_{i}$, density $\rho_{i}$, and velocity $\mathbf{v}_{i}, i=1, \ldots, N$. SPH is based on the approximation of a continuous function and its derivative:

$$
\begin{gathered}
f(\mathbf{r})=\sum_{j}^{N} \frac{m_{j}}{\rho_{j}} f\left(\mathbf{r}_{j}\right) W\left(\left|\mathbf{r}-\mathbf{r}_{j}\right|, h\right), \\
\nabla f(\mathbf{r})=\sum_{j}^{N} \frac{m_{j}}{\rho_{j}} f\left(\mathbf{r}_{j}\right) \nabla W\left(\left|\mathbf{r}-\mathbf{r}_{j}\right|, h\right),
\end{gathered}
$$

where the kernel $W\left(\left|\mathbf{r}-\mathbf{r}_{j}\right|, h\right)$ (Fig. 3.1) satisfies the normalization condition,

$$
\int W\left(\left|\mathbf{r}-\mathbf{r}_{j}\right|, h\right) d \mathbf{r}=1,
$$

and has compact support $h$. In the limit of $h \rightarrow 0, W$ approaches the Dirac delta function $\delta\left(\left|\mathbf{r}-\mathbf{r}_{j}\right|\right)$ :

$$
\lim _{h \rightarrow 0} W\left(\left|\mathbf{r}-\mathbf{r}_{j}\right|, h\right)=\delta\left(\left|\mathbf{r}-\mathbf{r}_{j}\right|\right) .
$$

A number of functional forms of $W$ have been used in the literature. In this study, we use $W$ in the form of a so-called "Wendland" kernel (Wendland; 1995):

$$
W\left(r_{i j}, h\right)=\alpha_{k}\left\{\begin{array}{ll}
\left(1-\frac{\mathbf{r}_{i j}}{h}\right)^{4}\left(4 \frac{\mathbf{r}_{i j}}{h}+1\right) & \text { if } 0 \leq r_{i j}<h \\
0 & \text { if } r_{i j} \geq h
\end{array},\right.
$$

where $\alpha_{k}=21 /\left(2 \pi h^{3}\right)$. 


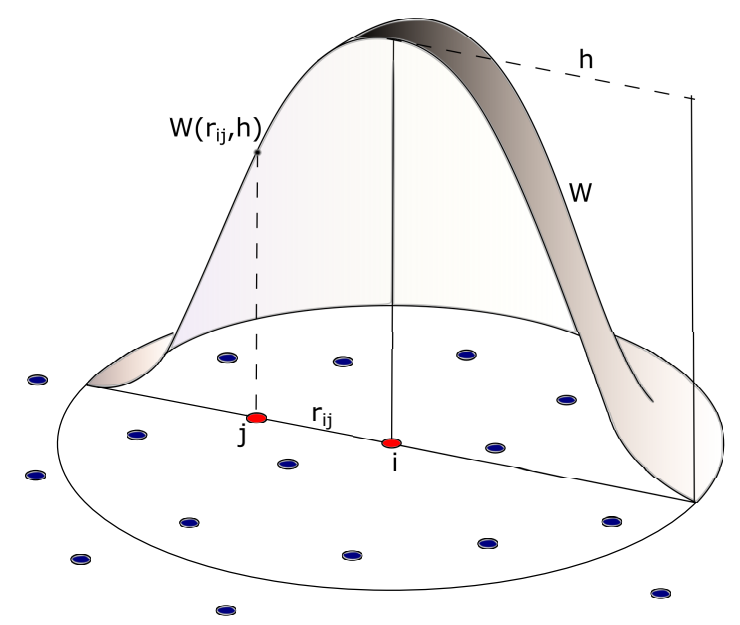

FIGURE 3.1: Kernel $W$ with a circular support domain of length $h$ and value $W\left(r_{i j}, h\right)$ between particles $i$ and $j$ at a distance $r_{i j}$.

The main equations, which we discretize with the PF-SPH method are the continuity equation (Chapters 4,5 , and 6 ),

$$
\frac{d \rho}{d t}=-\rho \nabla \cdot \mathbf{v}
$$

the momentum conservation equation (Chapters 4, 5, and 6),

$$
\frac{d \mathbf{v}}{d t}=-\frac{1}{\rho} \nabla P+\frac{\mu}{\rho} \nabla^{2} \mathbf{v}+\mathbf{g}
$$

and the Richards equation (Chapter 6),

$$
\frac{\partial \Theta(\psi)}{\partial t}=\left(C_{m}+\rho \mathbf{g S e} S_{s}\right) \frac{\partial \psi}{\partial t}=\nabla \cdot \mathbf{K}_{s} k_{r}(\psi) \nabla \psi+\frac{\partial K(\psi)}{\partial z} .
$$

The parameters are the pressure $P$, viscosity $\mu$, gravity $\mathbf{g}$, water content $\Theta$, hydraulic head $\psi$, specific storage coefficient $S_{s}, C_{m}$ specific moisture capacity, effective saturation $S_{e}$, relative hydraulic conductivity $k_{r}$, and saturated hydraulic conductivity $\mathbf{K}_{s}$.

In this framework, we consider two types of particles: (1) solid/boundary particles, which represent a solid surface, fracture walls and/or porous media, and (2) fluid/water particles. The solid particles are immobile, and placed on a uniform cubic lattice with a lattice size $\Delta x$. The spacing $\Delta x$ may vary depending on the simulation setup and on the required resolution. The fluid particles are initially placed on a uniform cubic lattice with the same $\Delta x$ as solid particles, or they can be randomly added to the simulation domain within a defined region during the simulation. Changes in positions of fluid particles are found via an SPH discretization of Eqs.(3.6)-(3.7):

$$
\frac{d \mathbf{r}_{i}}{d t}=\mathbf{v}_{i}
$$




$$
\begin{aligned}
\frac{d \mathbf{v}_{i}}{d t}=-\sum_{j=1}^{N} m_{j}\left(\frac{P_{j}}{\rho_{j}^{2}}+\frac{P_{i}}{\rho_{i}^{2}}\right) \frac{\mathbf{r}_{i j}}{r_{i j}} \cdot \frac{d W\left(r_{i j}, h\right)}{d r_{i j}}+2 \mu \sum_{j=1}^{N} m_{j} \frac{\mathbf{v}_{i j}}{\rho_{i} \rho_{j} r_{i j}} \cdot & \frac{d W\left(r_{i j}, h\right)}{d r_{i j}} \\
& +\mathbf{g}+\frac{1}{m_{i}} \sum_{j=1}^{N} \mathbf{F}_{i j},
\end{aligned}
$$

where the density $\rho_{i}$ is obtained from kernel summation as

$$
\rho_{i}=\sum_{j=1}^{N} m_{j} W\left(\mathbf{r}_{i j}, h\right) .
$$

This expression conserves mass exactly and, therefore, can be used instead of the mass conservation (continuity) Eq.(3.6).

The particle-particle interaction force $\mathbf{F}_{i j}$ in Eq. (3.10) is used to generate surface tension and the fluid wetting behavior. Here, we use $\mathbf{F}_{i j}$ in the form (Kordilla et al.; 2017; Kordilla J.; 2013):

$$
\mathbf{F}_{i j}=s_{i j}\left[\tilde{A}_{i j} \tilde{W}\left(r_{i j}, \frac{h}{2}\right) \frac{\mathbf{r}_{i j}}{r_{i j}}-\tilde{W}\left(r_{i j}, h\right) \frac{\mathbf{r}_{i j}}{r_{i j}}\right],
$$

where $\tilde{W}$ is a cubic spline function:

$$
\tilde{W}\left(r_{i j}, h\right)= \begin{cases}1-\frac{3}{2}\left(\frac{\mathbf{r}}{h}\right)^{2}+\frac{3}{4}\left(\frac{\mathbf{r}}{h}\right)^{3} & \text { if } 0 \leq \frac{\mathbf{r}}{h}<0.5 \\ \frac{1}{4}\left(2-\frac{\mathbf{r}}{h}\right)^{3} & \text { if } 0.5 \leq \frac{\mathbf{r}}{h}<1 \\ 0 & \text { if } \frac{\mathbf{r}}{h} \geq 1\end{cases}
$$

and $s_{i j}$ and $\tilde{A}_{i j}$ are parameters determining the magnitude of surface tension and the microscopic static contact angle. The parameter $s_{i j}$ is set to $s_{\mathrm{ff}}$ when particle $j$ is a fluid particle and $s_{\text {sf }}$ when particle $j$ is a solid particle (particle $i$ in Eq.(3.10) is always a fluid particle). For a liquid to wet a surface, $s_{\mathrm{ff}}$ should be set greater than $s_{\mathrm{sf}}$ and vice versa.

To evaluate pressure at each time step, we employ an equation of state (EOS) following Batchelor (1967) and Monaghan (2005):

$$
P=P_{0}\left\{\left(\frac{\rho}{\rho_{0}}\right)^{\gamma}-1\right\}
$$

where

$$
P_{0}=\frac{c^{2} \rho_{0}}{\gamma}
$$

Here, $\gamma=3$ (Chapter 4), and $\gamma=7$ (Chapters 5, 6), and the speed of sound $c$ is chosen such way, that the relative density fluctuation $|\delta \rho| / \rho$ is small enough (less than 3\%) to approximate an incompressible fluid (Morris et al.; 1997).

Chapters 4 and 5 deal with open surface and fracture flow without water exchange between fracture and matrix. Chapter 6 considers flow through a porous medium and at a fracture-matrix interface. The water exchange between solid and fluid particles is done via redistribution of initial water contents of particles and a particle removal algorithm. The fluid particles are initially fully saturated and have 
$\Theta_{f}=1.0$ and $\psi_{f}=0.0 \mathrm{~m}$. Depending on the type of problem, the solid particles are assigned $\Theta_{b}=0.0$ or $\Theta_{b}$ equal to a residual water content. Once fluid particles come into a contact with solid particles the exchange of fluid is governed by the Richards equation, i.e. a pressure-head dependent transfer is established. The changes in water content and pressure head for solid and fluid particles are found from Eq. (3.16):

$$
\frac{d \Theta_{i}}{d t}=\left(C_{m_{i}}+\rho_{i} \mathbf{g} S e_{i} S_{s}\right) \frac{d \psi_{i}}{d t}=\sum_{j=1}^{N} 2 \frac{m_{i} m_{j}}{m_{i}+m_{j}} \frac{\rho_{i}+\rho_{j}}{\rho_{i} \rho_{j}} \cdot \mathbf{K}_{s} k_{r_{i}}\left(d \psi_{i j}+d z_{i j}\right) \cdot \frac{d W\left(r_{i j}, h\right)}{d r_{i j}} .
$$

The maximum $\Theta_{b}$ of solid particles is equal to the saturated water content of the porous matrix based on the user defined porosity. If the water content $\Theta_{f}$ of fluid particles falls below a critical threshold $\Theta_{f}<0.99$, we redistribute the total water content of all particles below the threshold such that most particles are fully saturated again with $\Theta_{f}=1.0$. Fluid particles that are still below the critical threshold after the redistribution are marked and removed at the end of the time step. The residual water content (commonly less than the water content of one single particle) is stored and taken into account during the next time step. This procedure is carried out over all particles within a single MPI domain.

To properly conserve the water balance in the system, we rely on the mass conservation equation:

$$
\frac{\partial \Theta}{\partial t}=\nabla \cdot\left(\sum q_{\text {in }}-\sum q_{\text {out }}\right)=0,
$$

where $q$ is the specific flux. Every time step we calculate the sum of $\Theta_{f}$ and $\Theta_{b}$ of all fluid and boundary particles based on Eq. (3.18). To control the water balance in the system, the total $\Theta$ must stay constant:

$$
\Theta=\sum \Theta_{f}+\sum \Theta_{b}=\text { const. }
$$

\subsection{Simulation algorithm}

The three-dimensional SPH code is entirely written in Large-Scale Atomic/Molecular Massively Parallel Simulator (LAMMPS) (Ganzenmüller et al.; 2011). LAMMPS consists of various source files and classes, written in C/C++ (Plimpton; 1995). The classes, which are used, modified, and implemented in this study are shown in Fig. 3.2 within a class hierarchy and time stepping algorithm. Each boxed name refers to a class and has a pair of associated source files in lammps/src:

- The Memory class allocates all large vectors and arrays.

- The Error class checks for errors and prints warning messages.

- The Universe class sets up processors so that multiple simulations can be run, each on a subset of the processors allocated for a run.

- The Input class reads an input script, stores variables, and invokes standalone commands. The Read restart command reads all atom (particle) information (e.g., position, velocity, density, pressure head) from a previous simulation, allows to continue the simulation, and can be useful for long runs. The Read data command imports list of particles (e.g., coordinates) from other files, which allows to create complex rough fracture geometries separately from the main input script. The main input Variables are $\Delta x, h$, particle mass $m_{0}$ and initial 


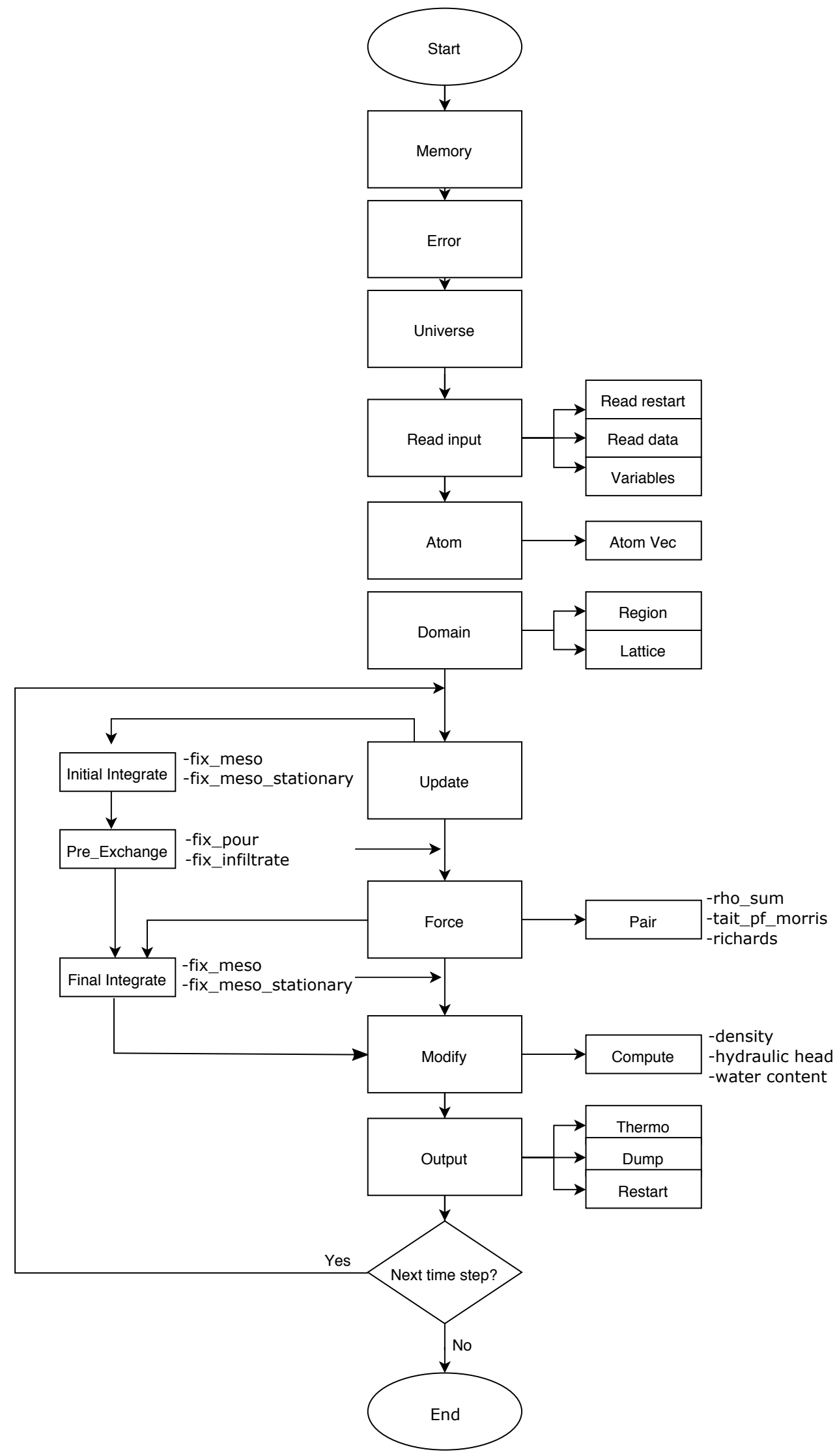

FIGURE 3.2: Simulation algorithm within the LAMMPS class hierarchy. 
density $\rho_{0}$ (Eqs. 3.10, 3.16), $\mu$, g (Eq. 3.10), $s_{s f}$ and $s_{f f}$ (Eq. 3.12), $c$ (Eq. 3.15), $\Theta_{0}, \psi_{0}, S_{s}, \mathbf{K}_{s}$ (Eq. 3.16), and a time step $\Delta t$.

- The Atom class stores all per-atom arrays. More precisely, they are allocated and stored by the AtomVec class, and the Atom class simply stores a pointer to them.

- The Domain class stores the simulation box geometry, as well as geometric Regions and any user definition of a Lattice (resolution/particle spacing $\Delta x$ ).

- The Update class takes care of the time stepping algorithm. We employ a modified Velocity Verlet time stepping scheme, where Initial Integrate calculates all variables at $1 / 2 \Delta t$, and Final Integrate at the end of $\Delta t$ :

$$
\begin{aligned}
\mathbf{v}_{i}\left(t+\frac{1}{2} \Delta t\right) & =\mathbf{v}_{i}+\frac{1}{2} \mathbf{a}_{i}(t) \\
\overline{\mathbf{v}}_{i}(t+\Delta t) & =\mathbf{v}_{i}(t)+\Delta t \mathbf{a}_{i} \\
\mathbf{r}_{i}(t+\Delta t) & =\mathbf{r}_{i}(t)+\Delta t \mathbf{v}_{i}\left(t+\frac{1}{2} \Delta t\right) \\
\text { calculation of } & \mathbf{a}_{i}(t+\Delta t) \text { using extrapolated velocity } \overline{\mathbf{v}}_{i} \\
\mathbf{v}_{i}(t+\Delta t) & =\mathbf{v}_{i}\left(t+\frac{1}{2} \Delta t\right)+\frac{1}{2} \mathbf{a}_{i}(t+\Delta t)
\end{aligned}
$$

where $\mathbf{a}_{i}=\frac{\mathbf{f}_{i}}{m_{i}}$ is the acceleration. Time step constraints are given by Tartakovsky and Meakin (2005):

$$
\begin{aligned}
& \Delta t \leq 0.25 h / 3 c \\
& \Delta t \leq 0.25 \min \left(h / 3\left|\mathbf{a}_{i}\right|\right)^{1 / 2} \\
& \Delta t \leq \min \left(\rho_{i} h^{2} / 9 \mu_{i}\right),
\end{aligned}
$$

where $\left|\mathbf{a}_{i}\right|$ is the magnitude of acceleration $\mathbf{a}_{i}$.

The information about fluid particles (e.g., positions, velocities) is calculated in $f i x \_m e s o$ (Initial Integrate) for $1 / 2 \Delta t$, and in $f i x \_m e s o$ (Final Integrate) for $\Delta t$. The information about solid particles (e.g., hydraulic heads, water con-

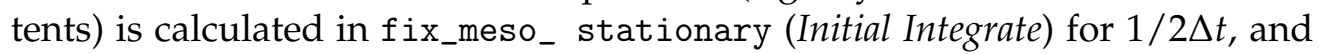
in fix_meso_stationary (Final Integrate) for $\Delta t$. The Pre_Exchange class inserts or deletes particles from the system; fix_pour inserts new fluid particles with a user defined volumetric flux; fix_infiltrate removes fluid particles if they are desaturated (Eqs. 3.17 and 3.18).

- The Force class computes various forces between atoms. The Pair parent class is for non-bonded or pair-wise forces; rho_sum computes densities (Eq. 3.11), tait_pf_morris computes forces in Eq. (3.7), and richards computes changes in water contents (Eq. 3.8).

- The Modify class stores lists of Fix and Compute classes, both of which are parent styles. The Compute class allows to compute all additional variables, which user would like to output.

- The Output class is used to generate 3 kinds of output from a LAMMPS simulation: information printed to the screen and log file, dump file snapshots, which can be opened with a visualization tool (e.g., Visit, Paraview), and restart files, which can be used to continue the simulation. These correspond to the Thermo, Dump, and Restart classes respectively. 
- The Timer class holds MPI timing information.

\section{Bibliography}

Batchelor, G. K. (1967). An introduction to fluid dynamics, Cambridge University Press, Cambridge, UK.

Ganzenmüller, G. C., Steinhauser, M. O., Van Liedekerke, P. and Leuven, K. U. (2011). The implementation of smooth particle hydrodynamics in LAMMPS.

Kordilla, J., Noffz, T., Dentz, M., Geyer, T. and Tartakovsky, A. M. (2017). Effect of unsaturated flow modes on partitioning dynamics of gravity-driven flow at a simple fracture intersection: laboratory study and three-dimensional smoothed particle hydrodynamics simulations., Water Resources Research 53(11): 9496-9518.

Kordilla J., Tartakovsky A.M., G. T. (2013). A smoothed particle hydrodynamics model for droplet and film flow on smooth and rough fracture surfaces, Advances in Water Resources 59: 1-14.

Monaghan, J. J. (2005). Smoothed particle hydrodynamics, Reports on progress in physics 68(8).

Morris, J. P., Fox, P. J. and Zhu, Y. (1997). Modeling low Reynolds number incompressible flows using SPH, Journal of Computational Physics 136(1): 214-226.

Plimpton, S. (1995). Fast parallel algorithms for short-range molecular dynamics, Computational Physics 117(1): 1-19.

Tartakovsky, A. and Meakin, P. (2005). Modeling of surface tension and contact angles with smoothed particle hydrodynamics, Physical Review E 72(2).

Wendland, H. (1995). Piecewise polynomial, positive definite and compactly supported radial functions of minimal degree., Advances in Computational Mathematics 4(1): 389-396. 



\title{
Chapter 4
}

\section{Smoothed particle hydrodynamics study of the roughness effect on contact angle and droplet flow ${ }^{1}$}

\begin{abstract}
.
We employ a pairwise force Smoothed particle hydrodynamics (PF-SPH) model to simulate sessile and transient droplets on rough hydrophobic and hydrophilic surfaces. PF-SPH allows modeling of free surface flows without discretizing the air phase, which is achieved by imposing the surface tension and dynamic contact angles with pairwise interaction forces.

We use the PF-SPH model to study the effect of surface roughness and microscopic contact angle on the effective contact angle and droplet dynamics. In the first part of this work, we investigate static contact angles of sessile droplets on different types of rough surfaces. We find that the effective static contact angles of Cassie and Wenzel droplets on a rough surface are greater than the corresponding microscale static contact angles. As a result, microscale hydrophobic rough surfaces also show effective hydrophobic behavior. On the other hand, microscale hydrophilic surfaces may be macroscopically hydrophilic or hydrophobic, depending on the type of roughness. We study the dependence of the transition between Cassie and Wenzel states on roughness and droplet size, which can be linked to the critical pressure for the given fluid-substrate combination. We observe good agreement between simulations and theoretical predictions.

Finally, we study the impact of the roughness orientation (i.e., an anisotropic roughness) and surface inclination on droplet flow velocities. Simulations show that droplet flow velocities are lower if the surface roughness is oriented perpendicular to the flow direction. If the predominant elements of surface roughness are in alignment with the flow direction, the flow velocities increase compared to smooth surfaces, which can be attributed to the decrease in fluid-solid contact area similar to the lotus effect. We demonstrate that classical linear scaling relationships between Bond and capillary number for droplet flow on flat surfaces also hold for flow on rough surfaces.
\end{abstract}

\subsection{Introduction}

Surface roughness and fluid-surface interactions control wettability and flow dynamics of droplets. Droplets are likely to spread on hydrophilic smooth surfaces to

\footnotetext{
${ }^{1}$ Shigorina, E., Kordilla, J., and Tartakovsky, A. M. (2017): Smoothed particle hydrodynamics study of the roughness effect on contact angle and droplet flow. Physical Review E, 96(3):033115.
} 
form a thin film or puddle and commonly form a spherical shape on fully hydrophobic smooth surfaces when droplet sizes are comparable to the capillary length of water (Quéré et al.; 2004). A surface is considered hydrophobic if the static contact angle is larger than $90^{\circ}$ and hydrophilic, otherwise. On smooth surfaces, the static contact angle $\theta_{0}$ only depends on the fluid-solid molecular interactions. Therefore, in this paper, we will refer to this as the microscopic contact angle $\theta_{0}$. On rough surfaces, the static contact angle, which we call the effective contact angle $\theta_{\text {eff }}$, depends on both the fluid-solid molecular interactions (and $\theta_{0}$ ) and surface roughness. Various authors have experimentally investigated the dependence of the contact angles on the chemical composition and roughness of solid surfaces (e.g., Genzer and Efimenko; 2006; Voigt and Gorb; 2009; Zhao et al.; 2007). Recently, molecular dynamics (MD) simulations have been used to study the effect of nanoscale roughness on static contact angles of droplets (Daub et al.; 2010; Yang et al.; 2008). It was shown that smooth hydrophilic surfaces can become less hydrophilic if certain types of roughness are added. In some cases, a superhydrophobic rough surface with a contact angle of $180^{\circ}$ can be created.

Droplet flow on rough surfaces has been investigated experimentally and numerically using MD simulations by Huang et al. (2009), Byun et al. (2008), Zhang et al. (2014), and Stamatopoulos et al. (2016). For example, Zhang et al. (2014) experimentally studied the droplet velocities on grooved surfaces with various inclination angles and different orientations of grooves relative to the flow direction. Results indicated that droplets experience less resistance to flow if grooves are oriented parallel to the flow direction, and they move significantly faster. On the other hand, water droplets barely moved when the grooves were oriented perpendicular to the flow direction.

In this work, we investigate contact angle dynamics of sessile and transient droplets on rough hydrophobic and hydrophilic surfaces using the pairwise force Smoothed particle hydrodynamics (PF-SPH) method implemented in LAMMPS (Plimpton; 1995), a massively parallel library for particle simulations. In PF-SPH, the boundary conditions at the fluid-fluid and fluid-fluid-solid interfaces are modeled by pairwise forces (Tartakovsky and Panchenko; 2016). In contrast to other numerical methods for multiphase flows (e.g., Huang et al.; 2009), PF-SPH allows for discretizing only the liquid phase in liquid-gas flows, which significantly reduces the computational cost for modeling water droplet flows where most of the domain usually is occupied by air.

A validation of the PF-SPH method for fluid-fluid systems (where both fluids are explicitly modeled) for modeling fluid-fluid and fluid-fluid-solid interfaces, including dynamic contact angles, with respect to Young-Laplace Voinov (1976) and Tanner (1979) laws was presented in Tartakovsky and Panchenko (2016). Similarly, here, we demonstrate the accuracy of PF-SPH for liquid-gas systems where only the liquid phase is explicitly modeled. Furthermore, the model is shown to reproduce the Cassie-to-Wenzel transition based on critical capillary pressure and internal droplet pressure. Next, we use the PF-SPH model to simulate highly intermittent, gravity-driven free surface flows for a diverse range of wetting conditions on time and length scales that are inaccessible to MD. We also use the PF-SPH method to study the effect of roughness on the effective static contact angle. We construct four surface geometries to investigate the changes of static contact angles of sessile droplets: rectangular, dual-rectangular, sinusoidal, and dual-sinusoidal surfaces.

Similar to the experimental work of Zhang et al. (2014), we study the effect of surface roughness orientation relative to the flow direction on the motion of water 
droplets and observe good qualitative agreement with our simulations. Grooves oriented parallel to the flow direction result in higher droplet velocities, while they impede movement when oriented perpendicular to the flow direction. We cast our results in a dimensionless form to investigate the relationship between Bond (Bo) and capillary $(\mathrm{Ca})$ numbers for different surface inclination angles and types of roughness. Simulations show that linear scaling relationships between Bo and Ca numbers for droplet flow on smooth surfaces (Podgorski et al.; 2001) also hold on rough surfaces.

\subsection{Governing equations and PF-SPH method}

We consider flow of water and air phases, where the air phase is continuous. Under this condition, it is common to disregard the effect of the air phase on water flow and model the latter by a combination of the continuity equation,

$$
\frac{d \rho}{d t}=-\rho \nabla \cdot \mathbf{v},
$$

and the momentum conservation equation,

$$
\frac{d \mathbf{v}}{d t}=-\frac{1}{\rho} \nabla P+\frac{\mu}{\rho} \nabla^{2} \mathbf{v}+\mathbf{g},
$$

subject to the free surface boundary condition at the fluid-air interface,

$$
-P \mathbf{n}=-\boldsymbol{\tau}_{w} \cdot \mathbf{n}+S \sigma \mathbf{n},
$$

and a no-slip boundary condition at the fluid-solid boundary. Here, $\boldsymbol{\tau}_{w}=[\mu(\nabla \mathbf{v}+$ $\left.\nabla \mathbf{v}^{\mathrm{T}}\right)$ ] is the viscous stress tensor, $\mathbf{v}$ is the velocity, $P$ is the pressure, $\mu$ is the viscosity, $\mathbf{g}$ is the gravitational acceleration, $S$ is the interface curvature, $\sigma$ is the surface tension, and the normal vector $\mathbf{n}$ points away from the non-wetting phase. In addition, the microscopic contact angle needs to be specified at the water-air-solid contact line.

In this work, we use the weakly compressible PF-SPH method (Kordilla J.; 2013; Tartakovsky and Meakin; 2005) to solve Eqs.(4.1)-(4.3). The PF-SPH discretization of Eqs. (4.2) and (4.3) is

$$
\begin{aligned}
\frac{d \mathbf{v}_{i}}{d t}=-\sum_{j=1}^{N} m_{j}\left(\frac{P_{j}}{\rho_{j}^{2}}+\frac{P_{i}}{\rho_{i}^{2}}\right) \frac{\mathbf{r}_{i j}}{r_{i j}} \cdot \frac{d W\left(r_{i j}, h\right)}{d r_{i j}}+2 \mu \sum_{j=1}^{N} m_{j} \frac{\mathbf{v}_{i j}}{\rho_{i} \rho_{j} r_{i j}} \cdot \frac{d W\left(r_{i j}, h\right)}{d r_{i j}} & \\
& +\mathbf{g}+\frac{1}{m_{i}} \sum_{j=1}^{N} \mathbf{F}_{i j},
\end{aligned}
$$

where $m_{i}$ and $m_{j}$ is a mass of particle $i$ or $j$, respectively, $W\left(r_{i j}, h\right)$ is the kernel function. A number of functional forms of $W$ have been used in the literature. Here, we use $W$ in the form of a so-called "Wendland" kernel (Wendland; 1995):

$$
W=\alpha_{k}\left\{\begin{array}{ll}
\left(1-\frac{|\mathbf{r}|}{h}\right)^{3} & \text { if } 0 \leq|\mathbf{r}|<h \\
0 & \text { if }|\mathbf{r}| \geq h
\end{array},\right.
$$

where $\alpha_{k}=168 / 16 \pi h^{3}$. 
The particle positions are advanced according to:

$$
\frac{d \mathbf{r}_{i}}{d t}=\mathbf{v}_{i}
$$

The particle-particle interaction force $\mathbf{F}_{i j}$ in Eq. (4.4) is used to generate surface tension and the fluid wetting behavior. Here, we use $\mathbf{F}_{i j}$ in the form:

$$
\mathbf{F}_{i j}=s_{i j}\left[\tilde{A}_{i j} \tilde{W}\left(r_{i j}, \frac{h}{2}\right) \frac{\mathbf{r}_{i j}}{r_{i j}}-\tilde{W}\left(r_{i j}, h\right) \frac{\mathbf{r}_{i j}}{r_{i j}}\right],
$$

where $\tilde{W}$ is a cubic spline function:

$$
\tilde{W}\left(r_{i j}, h\right)= \begin{cases}1-\frac{3}{2}\left(\frac{\mathbf{r}}{h}\right)^{2}+\frac{3}{4}\left(\frac{\mathbf{r}}{h}\right)^{3} & \text { if } 0 \leq \frac{\mathbf{r}}{h}<0.5 \\ \frac{1}{4}\left(2-\frac{\mathbf{r}}{h}\right)^{3} & \text { if } 0.5 \leq \frac{\mathbf{r}}{h}<1 \\ 0 & \text { if } \frac{\mathbf{r}}{h} \geq 1\end{cases}
$$

and $s_{i j}$ and $\tilde{A}_{i j}$ are parameters determining the magnitude of surface tension and the microscopic static contact angle. To impose the no-slip boundary condition away from the fluid-fluid-solid contact line and the contact angle at the contact line, the solid phase is discretized with a set of static "solid" particles, and summation in Eq. (4.4) is performed over both fluid and solid particles. The parameter $s_{i j}$ is set to $s_{\mathrm{ff}}$ when particle $j$ is a fluid particle and $s_{\text {sf }}$ when particle $j$ is a solid particle (particle $i$ in Eq. (4.4) is always a fluid particle). For a liquid to wet a surface, $s_{\mathrm{ff}}$ should be set greater than $s_{\text {sf }}$ and vice versa. In this work, the parameter $\tilde{A}_{i j}$ is set to $\tilde{A}_{\text {ff }}=8$ for interactions between two fluid particles and to $\tilde{A}_{\text {sf }}=24$ for interactions between fluid and solid particles.

The density is obtained from kernel summation as:

$$
\rho_{i}=\sum_{j=1}^{N} m_{j} W\left(\mathbf{r}_{i j}, h\right) .
$$

To evaluate pressure at each time step, we employ an equation of state (EOS) following Batchelor (1967) and Monaghan (2005):

$$
P=P_{0}\left\{\left(\frac{\rho}{\rho_{0}}\right)^{\gamma}-1\right\},
$$

where

$$
P_{0}=\frac{c^{2} \rho_{0}}{\gamma} .
$$

Here, $\gamma=3$ and the speed of sound $c$ are chosen so that the relative density fluctuation $|\delta \rho| / \rho$ is small enough (less than $3 \%$ ) to approximate an incompressible fluid (Morris et al.; 1997). To integrate Eq. (4.4), we employ a modified Velocity Verlet time 
stepping scheme:

$$
\begin{aligned}
& \mathbf{v}_{i}\left(t+\frac{1}{2} \Delta t\right)=\mathbf{v}_{i}+\frac{1}{2} \mathbf{a}_{i}(t) \\
& \overline{\mathbf{v}}_{i}(t+\Delta t)=\mathbf{v}_{i}(t)+\Delta t \mathbf{a}_{i} ; \\
& \mathbf{r}_{i}(t+\Delta t)=\mathbf{r}_{i}(t)+\Delta t \mathbf{v}_{i}\left(t+\frac{1}{2} \Delta t\right) \\
& \text { calculation of } \mathbf{a}_{i}(t+\Delta t) \text { using extrapolated velocity } \overline{\mathbf{v}}_{i} ; \\
& \mathbf{v}_{i}(t+\Delta t)=\mathbf{v}_{i}\left(t+\frac{1}{2} \Delta t\right)+\frac{1}{2} \mathbf{a}_{i}(t+\Delta t)
\end{aligned}
$$

where $\mathbf{a}_{i}=\frac{\mathbf{f}_{i}}{m_{i}}$ is the acceleration.

Time step constraints are given by Tartakovsky and Meakin (2005):

$$
\begin{aligned}
& \Delta t \leq 0.25 h / 3 c \\
& \Delta t \leq 0.25 \min \left(h / 3\left|\mathbf{a}_{i}\right|\right)^{1 / 2} \\
& \Delta t \leq \min \left(\rho_{i} h^{2} / 9 \mu_{i}\right)
\end{aligned}
$$

where $\left|\mathbf{a}_{i}\right|$ is the magnitude of acceleration $\mathbf{a}_{i}$.

In our simulations, we set the density and viscosity of water to $\rho_{0}=1000 \mathrm{~kg} / \mathrm{m}^{3}$ and $\mu=0.001296 \mathrm{~Pa}$ s, respectively. Initially, the SPH particles are placed on a uniform cubic lattice with the lattice size $0.5 \times 10^{-4} \mathrm{~m}$ (unless mentioned otherwise), which results in a fluid particle mass of $m_{0}=1.25 \times 10^{-10} \mathrm{~kg}$. The mass of solid particles is set to that of the fluid particle. The smoothing length is set to $h=$ $1.71 \times 10^{-4} \mathrm{~m}$, the speed of sound to $c=4.5 \mathrm{~m} \mathrm{~s}^{-1}$, and the gravitational acceleration to $\mathbf{g}=9.81 \mathrm{~m} / \mathrm{s}^{2}$.

\subsection{Model parametrization and verification}

\subsubsection{Surface tension}

The parameter $s_{\mathrm{ff}}$ is calibrated with respect to the surface tension of water by simulating a droplet and using the Young-Laplace law to relate the difference of pressure inside and outside of the bubble, $\Delta P$ and its radius, $R_{\text {eq }}$, to the surface tension $\sigma$ :

$$
\sigma=\frac{R_{\mathrm{eq}}}{2} \Delta P .
$$

Because the pressure outside of the bubble is zero, $\Delta P$ is equal to the pressure inside the bubble. It should be noted that the total pressure in PF-SPH is a sum of the pressure prescribed via the EOS and generated by $\mathbf{F}_{i j}$. As in any particle system, the total pressure generated by SPH particles can be calculated from the virial formula (Allen and Tildesley; 1989; Kordilla J.; 2013; Tartakovsky and Meakin; 2005):

$$
P_{T}=\frac{1}{2 d V_{r}} \sum_{i} \sum_{j} \mathbf{r}_{i j} \mathbf{f}_{i j}=\frac{1}{8 r_{v}^{3}} \sum_{i} \sum_{j} \mathbf{r}_{i j} \mathbf{f}_{i j}
$$

where $d=3$ for a three-dimensional system and $\sum_{j} \mathbf{f}_{i j}=m_{i} d \mathbf{v}_{i} / d t$. The double summation is performed over all particles within the distance $r_{v}$ from the droplet center, where $r_{v}=R_{\text {eq }}-h$, to exclude the boundary deficiency effect. We obtain the surface tension of water with $s_{\mathrm{ff}}=3.5 \times 10^{-6}$. Six liquid droplets with radii ranging from $0.5 \mathrm{~mm}$ to $1 \mathrm{~mm}$ are simulated in the absence of gravity with $s_{\mathrm{ff}}=3.5 \times 10^{-6}$ and the other parameters as described. Figure 4.1 shows the fluid pressure $P_{T}$ in the 
center of the equilibrated liquid droplet versus $1 / R_{\text {eq }}$. The surface tension, found as half of the slope of the straight line fitted through the simulation results, is $\sigma=$ $73.14 \mathrm{mN} \mathrm{m}^{-1}$ (the water surface tension is $72 \mathrm{mN} \mathrm{m}^{-1}$ at $25 \mathrm{C}$ ).

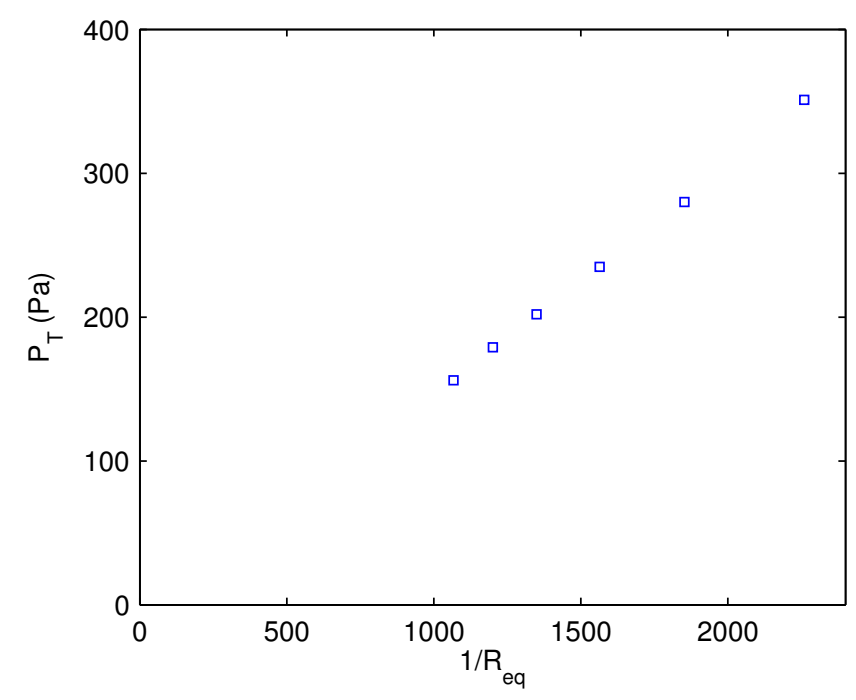

FIGURE 4.1: Pressure for various droplet sizes.

\subsubsection{Static contact angles on smooth surface}

To measure static contact angles, we simulate droplets that are slowly brought into contact with the flat surface. Each droplet has a volume of $V=2.14 \mathrm{~mm}^{3}$. After droplets reach equilibrium and remain static, we select fluid particles at the intersection of the droplet surface with the $x y$ - and $z y$-planes and fit circles with radius $R_{x}$ in the $x y$-plane and $R_{z}$ in the $z y$-plane as shown in Fig. 4.2. The contact angles $\theta_{0}^{x}$ in the $x$ direction and $\theta_{0}^{z}$ in the $z$ direction can be found as

$$
\theta_{0}^{x, z}=90 \pm \arcsin \left(\frac{l_{x, z}}{R_{x, z}}\right)
$$

where $l_{x, z}$ is a distance between circle center and solid surface. In Eq. (4.16), the addition is carried out for static contact angles larger than $90^{\circ}$ and subtraction otherwise. The static contact angle $\theta_{0}$ is equal to the arithmetic mean of $\theta_{0}^{x}$ and $\theta_{0}^{z}$.

For the parameter set described above, the (microscopic) static contact angle $\theta_{0}$ on a smooth surface depends on the interaction forces $s_{\text {sf }}$ between solid and fluid particles (Table 4.1). Figure 4.3 shows that $\theta_{0}$ decreases with increasing $s_{\text {sf }}$. All static contact angles $\theta_{0}$ are measured with a standard error $S E_{\bar{\theta}_{0}} \approx \pm 0.2^{\circ}$, which is computed as

$$
S E_{\overline{\theta_{0}}}=\frac{S}{\sqrt{5}}
$$

where $s$ is the standard deviation of the mean $\overline{\theta_{0}}$ of 5 droplets. Droplets are brought into contact with the solid surface from five different distances, in order to randomize the dynamic contact line movement until a static contact angle is achieved.

To investigate the pinning effect due to the discrete nature of the solid surface we compute the difference $\varepsilon_{0}$ in contact angles in the $x$ and $z$ directions:

$$
\varepsilon_{0}=\left|\theta_{0}^{x}-\theta_{0}^{z}\right|
$$




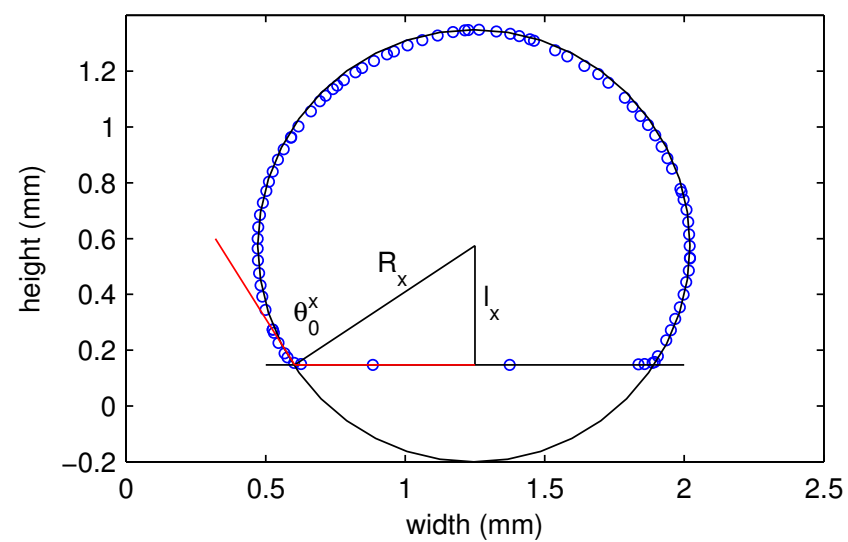

FIGURE 4.2: Static contact angle measurements. Here, only the droplet cross-section in the $x y$-plane is shown.

TABLE 4.1: Static contact angles of droplets for different solid-fluid interaction strengths $s_{\mathrm{sf}}$.

\begin{tabular}{lllllll}
\hline$s_{\text {sf }}$ & 0 & $1 \times 10^{-6}$ & $1.3 \times 10^{-6}$ & $1.8 \times 10^{-6}$ & $2.2 \times 10^{-6}$ & $2.8 \times 10^{-6}$ \\
\hline$\theta_{0}^{x}$ & $122.4^{\circ}$ & $100.8^{\circ}$ & $85.2^{\circ}$ & $81.3^{\circ}$ & $77.2^{\circ}$ & $0.1^{\circ}$ \\
$\theta_{0}^{z}$ & $122.9^{\circ}$ & $101.2^{\circ}$ & $84.3^{\circ}$ & $81.7^{\circ}$ & $77.7^{\circ}$ & $72.2^{\circ}$ \\
$\theta_{0}$ & $122.7^{\circ}$ & $100.9^{\circ}$ & $84.7^{\circ}$ & $81.5^{\circ}$ & $77.5^{\circ}$ & $72.8^{\circ}$ \\
$\varepsilon_{0}$ & $0.5^{\circ}$ & $0.4^{\circ}$ & $0.9^{\circ}$ & $0.4^{\circ}$ & $0.5^{\circ}$ & $0.8^{\circ}$ \\
\hline
\end{tabular}

The values of $\varepsilon_{0}$ are reported in Table 4.1. In these simulations $\varepsilon_{0}$ is less than $1^{\circ}$, and we assume that pinning effects are negligible for the chosen resolution.

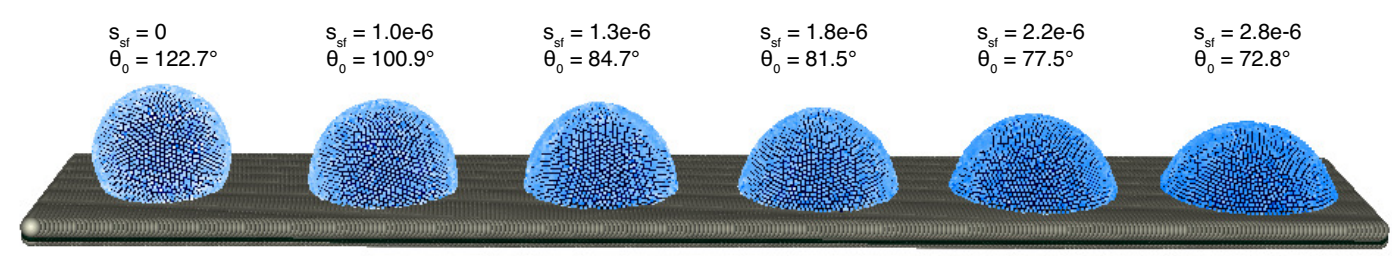

FIGURE 4.3: Static contact angles for different solid-fluid interaction strengths $s_{\text {sf }}$.

To validate our model, we simulate droplet spreading on a horizontal surface (Fig. 4.4, inset) and compare the time-dependent height of the droplet, $H$, with the Tanner law: $H \sim t^{-2 n / 3}$, where $n=0.3$ in three spatial dimensions (Tanner; 1979). The simulation is initialized by placing a droplet with an initial radius $R_{0}=1.2 \mathrm{~mm}$ on the horizontal surface. After equilibration of the droplet on the solid surface in the presence of gravity, we prescribe a solid-fluid interaction force of $s_{\mathrm{sf}}=3 \times 10^{-6}$ and measure the height changes of the droplet over time.

Figure 4.4 shows $H$ as a function of time obtained from the simulation with the exponent $n=0.274$, which is close to the theoretical value of $n=0.3$.

\subsubsection{Dynamic contact angles on smooth surface}

Here we demonstrate that the PF-SPH model predicts dynamic contact angles in accordance with the theoretical Cox-Voinov relationship (Bonn et al.; 2009; Voinov; 


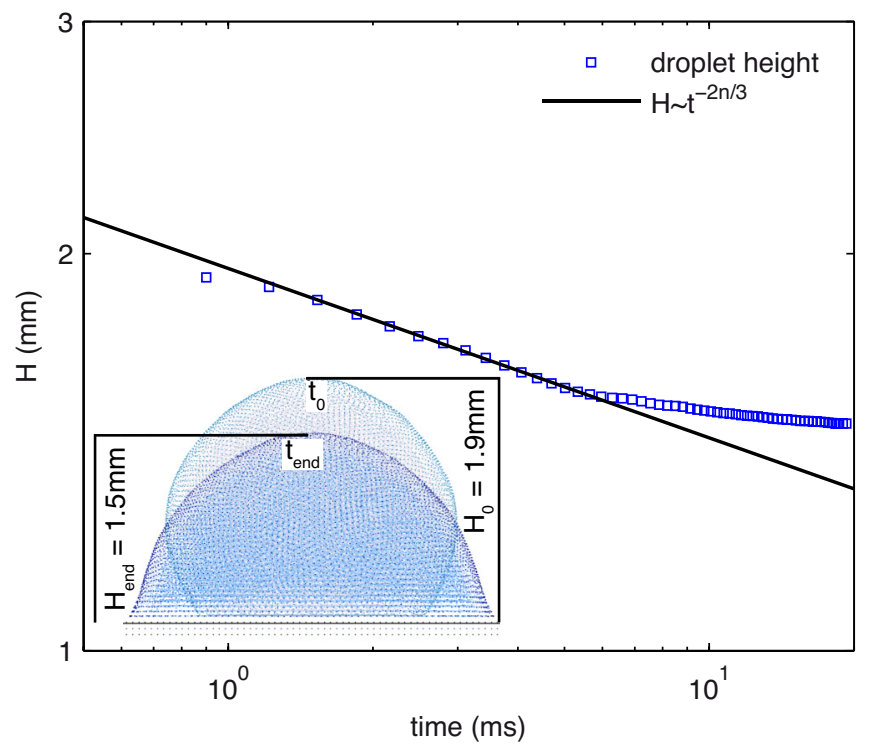

FIGURE 4.4: Model verification with respect to Tanner's law: height of the droplet as a function of time. The inset shows the droplet spreading on a horizontal surface.

1976). The dynamic contact angle as a function of the contact line velocity is measured by simulating a plate withdrawal from a pool of liquid. According to the CoxVoinov relationship, the receding contact angle scales with the capillary number, $\mathrm{Ca}$, as

$$
\theta_{0}^{3}-\theta_{r}^{3} \sim \mathrm{Ca},
$$

where $\mathrm{Ca}$ is defined as

$$
\mathrm{Ca}=\mu \frac{v}{\sigma},
$$

and $v$ is the velocity of the moving plate.

The simulation setup is shown in Fig. 4.5(inset). The receding angle is computed as the angle formed by a circle, fitted to the interface, and the solid boundary. From Fig. 4.5 we find that $\theta_{0}^{3}-\theta_{r}^{3} \sim \mathrm{Ca}^{\alpha}$ with $\alpha=0.9469$, which is close to the theoretical value $\alpha=1$.

Physically, $\theta_{0}$ depends on the chemical composition of fluids and the solid surface, and, numerically (in the PF-SPH model), $\theta_{0}$ is a function of the interaction parameters $s_{\mathrm{sf}}$ and $s_{\mathrm{ff}}$. Therefore, we refer to $\theta_{0}$ as a microscopic static contact angle. In the following, we study droplet behavior on rough surfaces obtained by "carving" a flat surface and characterize macroscopic wetting properties of these rough surfaces in terms of the effective contact angle formed by a droplet and a plane fitted to the rough surface.

\subsection{Wenzel and Cassie droplets on rough solid surfaces}

Depending on $\theta_{0}$ and surface roughness, a droplet on a rough surface can be in one of the three regimes: the Wenzel regime (Wenzel; 1936), the Cassie regime (Cassie and Baxter; 1944), or the mixed Cassie-Wenzel regime. Figure 4.6 shows the PFSPH simulations of a droplet in all three regimes. On "microscopically" hydrophilic rough surfaces (i.e., surfaces with $\theta_{0}>\pi / 2$ ), Wenzel drops are formed by the fluid 


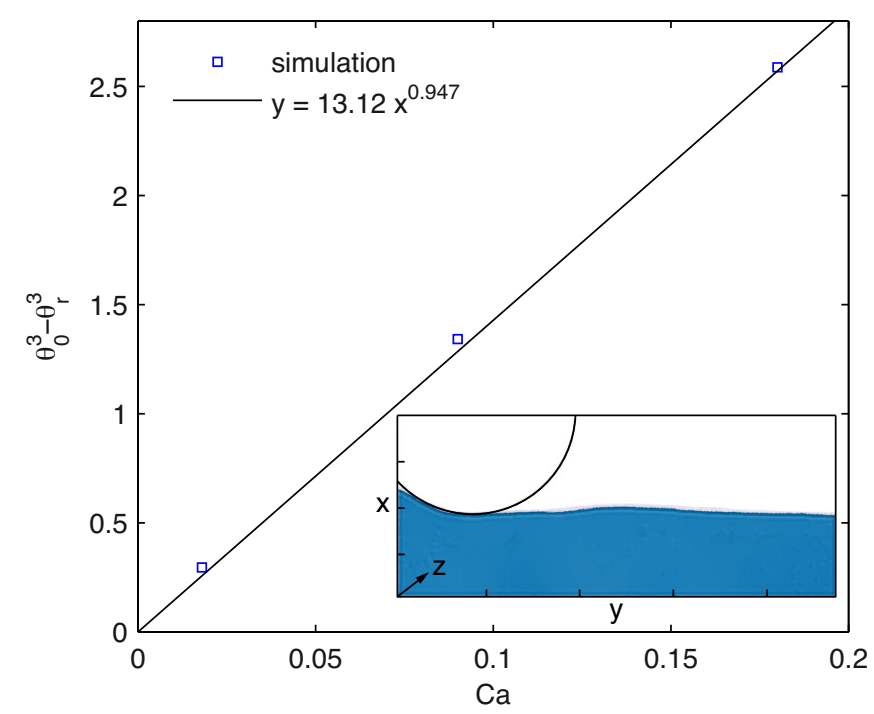

FIGURE 4.5: Cox-Voinov relationship for receding contact angles. The inset shows the simulation results of a plate withdrawal from a pool of liquid ( $x=25 \mathrm{~mm}, y=10 \mathrm{~mm})$.

filling surface indentations (Fig. 4.6, middle). On microscopically hydrophobic surfaces, depending on the ratio of roughness to the size and mass of the droplet, Cassie (Fig. 4.6, left) or Cassie-Wenzel regime (Fig. 4.6, right) droplets can form. In the Cassie regime, a droplet "rests" on the surface spikes, while a droplet partially filling the pits and depressions of a rough surface is considered to be in the Cassie-Wenzel regime. In general, the effective contact angle $\theta_{\text {eff }}$, formed by a droplet on a rough surface, differs from the microscopic static contact angle $\theta_{0}$.

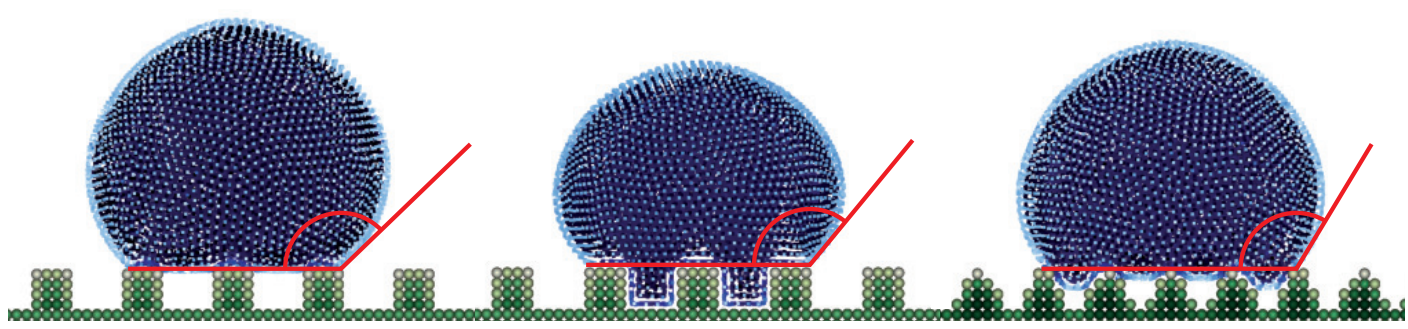

FIGURE 4.6: Different states of droplets depending on wetting conditions (left to right): Cassie state, Wenzel state, and Cassie-Wenzel state.

In the following, we simulate droplets in all three regimes and study the relationship between the roughness geometry, $\theta_{0}$ (or the parameter $s_{\mathrm{sf}}$ ), and $\theta_{\mathrm{eff}}$. We investigate both hydrophobic surfaces $\left(\theta_{0}>90^{\circ}\right)$ and hydrophilic $\left(\theta_{0}<90^{\circ}\right)$ surfaces.

\subsection{Effective contact angles of droplets on rough microscopi- cally hydrophobic surfaces}

Microscopically hydrophobic rough surfaces are modeled by setting $s_{\mathrm{sf}}=0$, which yields $\theta_{0}=122.7^{\circ}$. We consider four types of rough surfaces with rectangular, dualrectangular, sinusoidal, and dual-sinusoidal patterns (see Fig. 4.7). 

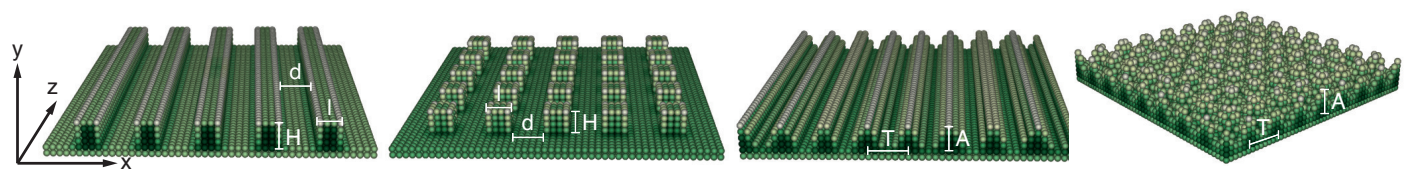

FIGURE 4.7: Surface parameters (four types, from left to right): (1) for rectangular surface - height $H$ and width $l$ of a bar; $d$ - distance between bars; (2) for dual-rectangular surface - height $H$ and $l$ width of a block, $d$ - distance between blocks; (3) for sinusoidal surface - period $T$ and magnitude $A$ of a sinusoidal function in the $x$ direction; (4) for dual-sinusoidal surface - period $T$ and magnitude $A$ of a sinusoidal function in the $x$ and $z$ directions.

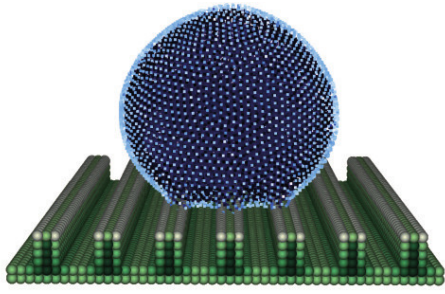

(a)

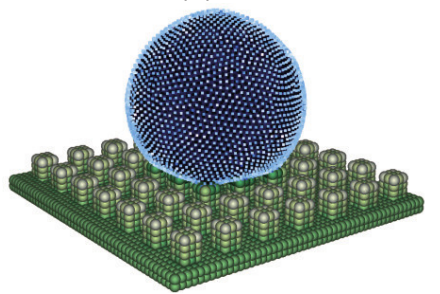

(d)

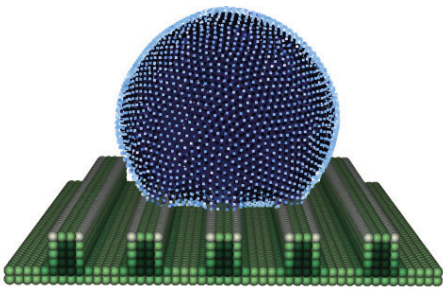

(b)

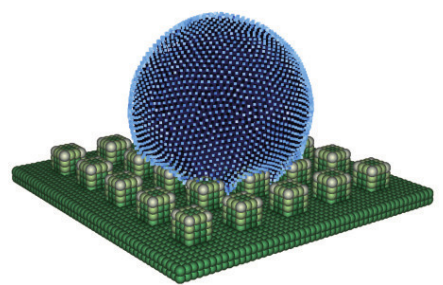

(e)

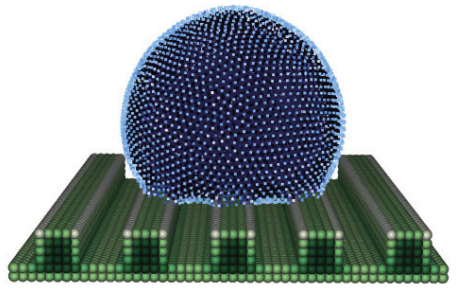

(c)

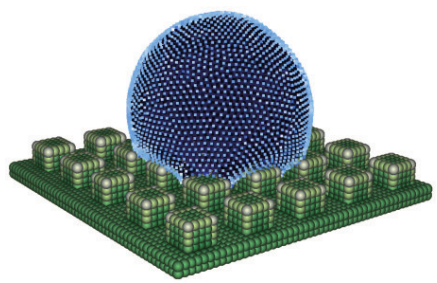

(f)

FIGURE 4.8: Static contact angles of droplets on hydrophobic rectangular $(\mathrm{a}-\mathrm{c})$ and dual-rectangular $(\mathrm{d}-\mathrm{f})$ surfaces. Surface parameters are: $d=0.2 \mathrm{~mm}, l=0.15 \mathrm{~mm}(\mathrm{a}, \mathrm{d}) ; d=0.25 \mathrm{~mm}, l=0.2 \mathrm{~mm}(\mathrm{~b}, \mathrm{e})$; $d=0.25 \mathrm{~mm}, l=0.25 \mathrm{~mm}(\mathrm{c}, \mathrm{f}) ; H=0.2 \mathrm{~mm}$ for all types of surfaces.

We model droplets with an initial radius $R=0.8 \mathrm{~mm}$, which are slowly brought into contact with a rough surface. After equilibration of a droplet on the rough surface, we measure the effective contact angle $\theta_{\text {eff }}$ in the $x$ - and $z$-directions as shown in Fig. 4.2. For Wenzel and Cassie-Wenzel droplets, which penetrate depressions of the rough surface, we measure $\theta_{\text {eff }}$ relative to the nominal smooth surface on top of the blocks, as indicated in Figs. 4.6(middle) and 4.6(right) by the solid line. Depending on their geometry, solid surfaces are discretized with approximately 20000 boundary particles and droplets with 17075 fluid particles. Simulations are run on eight processors.

\subsubsection{Rectangular and dual-rectangular surfaces}

Figure 4.7 (first from left) shows the rectangular-patterned surface. This surface is parametrized by the distance $d$ between "bars," the height $H$, and the width $l$ of the bars. We study three rectangular-patterned surfaces with different parameters $l$ and $d$, and $H=0.2 \mathrm{~mm}$ : a fine-roughness surface with small $d$ and $l$ (Fig. 4.8a), a medium-roughness surface (Fig. 4.8b), and a coarse-roughness surface with large $d$ and $l$ (Fig. $4.8 \mathrm{c}$ ). 
Fig. 4.7 (second from left) depicts the dual-rectangular surface. It is constructed of blocks of height $H$, length $l$, and the distance $d$ between the blocks. Figures $4.8 \mathrm{~d}-$ $4.8 \mathrm{f}$ show three types of dual-rectangular surfaces: a fine-roughness surface (Fig. $4.8 \mathrm{~d}$ ), medium-roughness surface (Fig. 4.8e), and a coarse-roughness dual-rectangular surface (Fig. 4.8f).

Figure 4.8 also shows the equilibrated droplets on rectangular-patterned surfaces. Table 4.2 provides the corresponding effective contact angle values. The effective static contact angle $\theta_{\mathrm{eff}}^{x}$ measured in the $x$ direction perpendicular to the bars increases with decreasing $l$ and/or increasing $d$. All droplets on hydrophobic rectangular surfaces are in a Cassie state. The effective static contact angle $\theta_{\text {eff }}^{z}$ of a droplet measured in the $z$ direction parallel to the ripples varies between $123.3^{\circ}$ and $125.5^{\circ}$, which is close to the corresponding $\theta_{0}=122.7^{\circ}$.

Due to the isotropic geometry of the dual-rectangular-patterned surfaces, $\theta_{\mathrm{eff}}^{x}$ and $\theta_{\text {eff }}^{z}$ are the same in both directions (Figs. 4.8d-4.8f). The largest contact angle $\theta_{\text {eff }}^{x} \approx$ $\theta_{\text {eff }}^{z} \approx 151^{\circ}$ is measured on a fine dual-rectangular surface (Fig. 4.8d). In contrast, the droplets on medium- and coarse-roughness surfaces are in the Wenzel state, even though not all small surface depressions are completely filled with fluid because of microscale surface hydrophobicity. The effective contact angles of "Wenzel" droplets are larger than the microscopic static contact angle, and the microscale hydrophobic rough surfaces also show macroscale hydrophobic behavior.

\subsubsection{Sinusoidal and dual-sinusoidal surfaces}

Here, we study the contact angles of droplets on sinusoidal surfaces with longitudinal ripples in the $z$ direction and a sinusoidal cross section in the $x$ direction (Fig. 4.7, third from left). The sinusoidal surfaces are parametrized as

$$
S(x, z)=\frac{A}{2} \cos \left(x \frac{2 \pi}{T}\right)+0.00015
$$

and the solid boundary in simulations is constructed by filling the region $y<S(x)$ with solid particles. The parameters of this surface are the period of the sinusoidal function $T$ and the magnitude $A$ in the $y$-direction, which is equal for all types of sinusoidal surfaces $A=0.2 \mathrm{~mm}$. We employ three types of rough sinusoidal surfaces: a fine-roughness surface with $T=0.2 \mathrm{~mm}$ (Fig. 4.9a), a medium-roughness surface with $T=0.25 \mathrm{~mm}$ (Fig. $4.9 \mathrm{~b}$ ), and a coarse-roughness sinusoidal surface with $T=0.3 \mathrm{~mm}$ (Fig. 4.9c).

The dual-sinusoidal surface is created as a surface with sinusoidal cross sections in the $x$ and $z$ directions (Fig. 4.7, forth from left), described by the equation:

$$
S(x, z)=\frac{A}{2} \cos \left(x \frac{2 \pi}{T}\right)+\frac{A}{2} \cos \left(z \frac{2 \pi}{T}\right)+0.00015 .
$$

The parameter $T$ is varied to create three surfaces: a fine-roughness surface with $T=0.2 \mathrm{~mm}$ (Fig. 4.9d), a medium-roughness surface with $T=0.25 \mathrm{~mm}$ (Fig. 4.9e), and a coarse-roughness dual-sinusoidal surface with $T=0.3 \mathrm{~mm}$ (Fig. 4.9f). The magnitude $A$ is equal to $0.2 \mathrm{~mm}$ for all three surfaces. In the simulations, the region $y<S(x, z)$ is filled with solid particles.

Figures 4.9 depict droplets on the sinusoidal and dual-sinusoidal surfaces and Table 4.2 shows the effective static contact angles. Here, the droplet on the fine sinusoidal surface is in the Cassie state, the droplet on the medium sinusoidal surface is in the Cassie-Wenzel state, and droplets on the coarse sinusoidal and dual-sinusoidal 


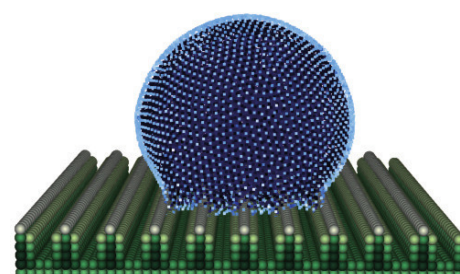

(a)

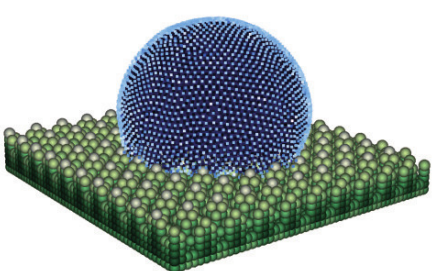

(d)

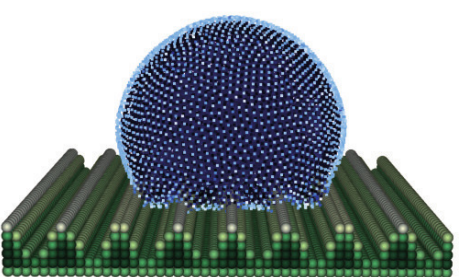

(b)

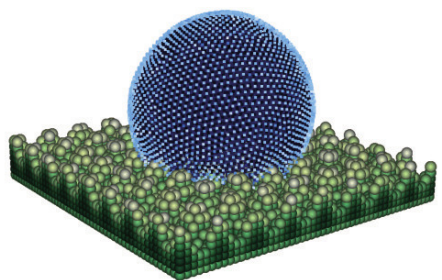

(e)

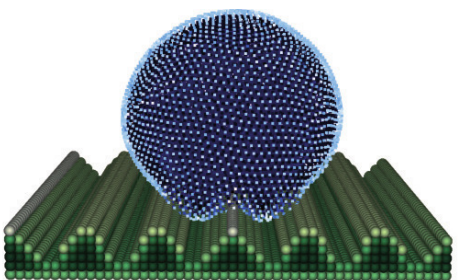

(c)

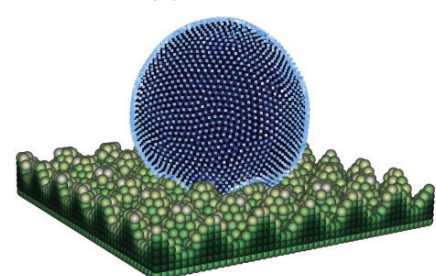

(f)

FIGURE 4.9: Static contact angles of droplets on hydrophobic sinusoidal $(\mathrm{a}-\mathrm{c})$ and dual-sinusoidal $(\mathrm{d}-\mathrm{f})$ surfaces. Surface parameters are: $A=0.2 \mathrm{~mm}, T=0.2 \mathrm{~mm}(\mathrm{a}, \mathrm{d}) ; A=0.2 \mathrm{~mm}, T=0.25 \mathrm{~mm}(\mathrm{~b}, \mathrm{e})$; $A=0.2 \mathrm{~mm}, T=0.3 \mathrm{~mm}(\mathrm{c}, \mathrm{f})$.

surfaces are macroscopically in the Wenzel state. For all considered microscopically hydrophobic rough surfaces, the effective static contact angle is greater than $90^{\circ}$, i.e., these surfaces are macroscopically hydrophobic.

\subsection{Effective contact angles of droplets on rough microscopi- cally hydrophilic surfaces}

The microscopic hydrophilic behavior of droplets on a solid surface is achieved by setting the solid-fluid interaction strength to $s_{\mathrm{sf}}=1.3 \times 10^{-6}$, which yields $\theta_{0}=$ 84.7 ${ }^{\circ}$. The surface geometries are the same as in the preceding section (see Fig. 4.7). We find that Wenzel droplets form on all considered microscopically hydrophilic surfaces (see Figs. 4.10 and 4.11).

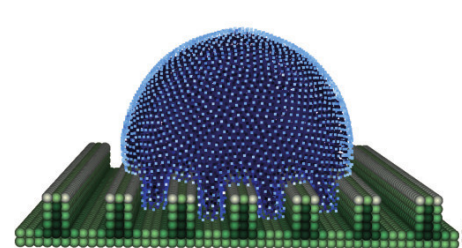

(a)

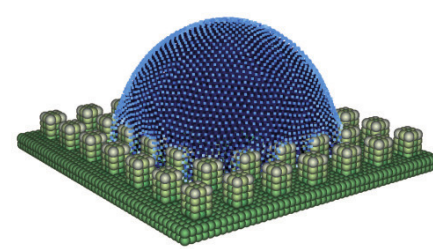

(d)

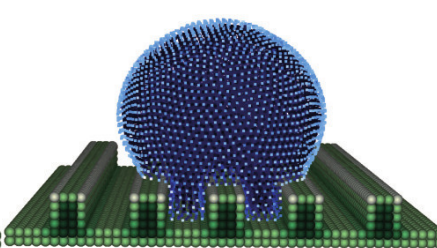

(b)

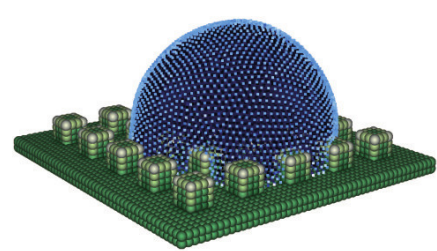

(e)

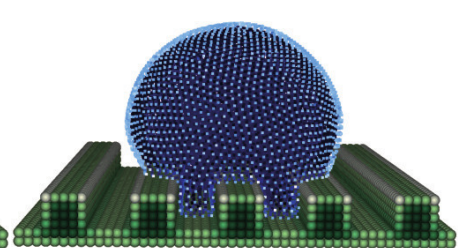

(c)

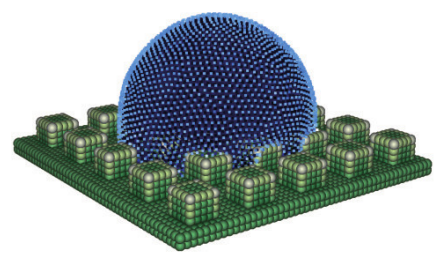

(f)

FIGURE 4.10: Static contact angles of droplets on hydrophilic rectangular $(\mathrm{a}-\mathrm{c})$ and dual-rectangular $(\mathrm{d}-\mathrm{f})$ surfaces. Surface parameters are: $d=0.2 \mathrm{~mm}, l=0.15 \mathrm{~mm}(\mathrm{a}, \mathrm{d}) ; d=0.25 \mathrm{~mm}, l=0.2 \mathrm{~mm}(\mathrm{~b}, \mathrm{e})$; $d=0.25 \mathrm{~mm}, l=0.25 \mathrm{~mm}(\mathrm{c}, \mathrm{f}) ; H=0.2 \mathrm{~mm}$ for all types of surfaces. 
Table 4.2 lists the resulting effective contact angles. The effective static contact angle of Wenzel droplets on the dual-rectangular and dual-sinusoidal microscopically hydrophilic surfaces are larger than $90^{\circ}$. This means that the dual-rectangular and dual-sinusoidal roughnesses considered in this work make microscopically hydrophilic surfaces macroscopically hydrophobic. For the rectangular-rough and sinusoidal-rough surfaces, the effective contact angles in the $x$ direction are greater than $90^{\circ}$, but the effective contact angles in the $z$ direction are smaller than the corresponding microscopic contact angle. These types of surfaces have mixed effective wettability, i.e., they are macroscopically hydrophilic in the $z$ direction and hydrophobic in the $x$ direction.

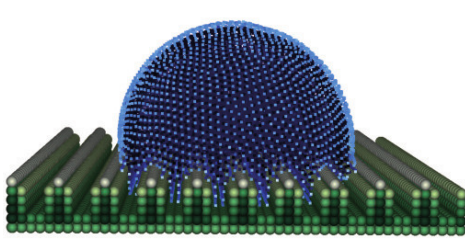

(a)

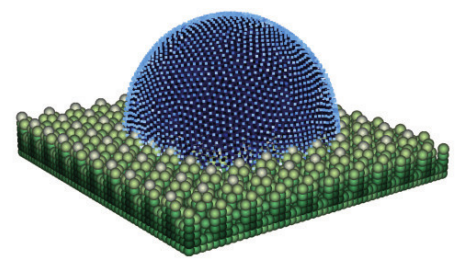

(d)

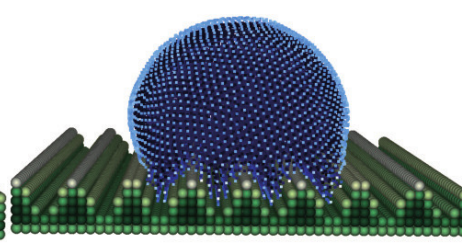

(b)

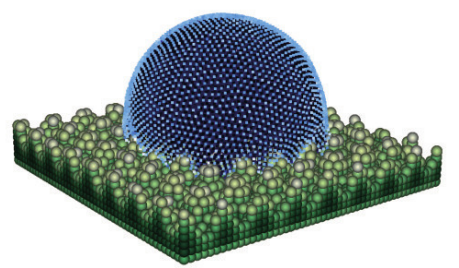

(e)

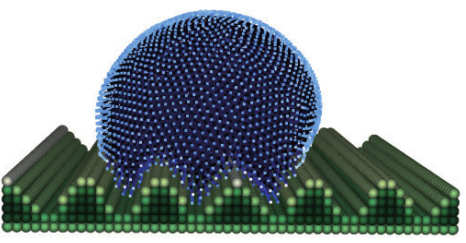

(c)

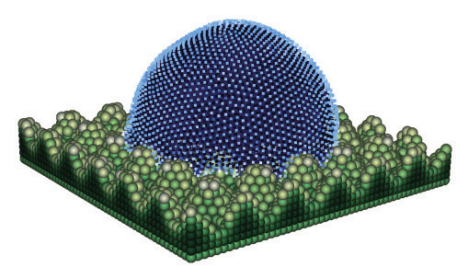

(f)

FIGURE 4.11: Static contact angles of droplets on hydrophilic sinusoidal $(\mathrm{a}-\mathrm{c})$ and dual-sinusoidal $(\mathrm{d}-\mathrm{f})$ surfaces. Surface parameters are: $A=0.2 \mathrm{~mm}, T=0.2 \mathrm{~mm}(\mathrm{a}, \mathrm{d}) ; A=0.2 \mathrm{~mm}, T=0.25 \mathrm{~mm}(\mathrm{~b}, \mathrm{e})$;

$$
A=0.2 \mathrm{~mm}, T=0.3 \mathrm{~mm}(\mathrm{c}, \mathrm{f}) \text {. }
$$

\subsection{Dimensionless analysis of effective static contact angles}

The influence of surface geometry on the effective contact angles of droplets can be described by the (dimensionless) scaling ratio $\lambda$. For rectangular and dual-rectangular surfaces, the scaling ratio $\lambda$ is defined as

$$
\lambda=\frac{l d}{R_{0}^{2}}
$$

and for sinusoidal and dual-sinusoidal surfaces as

$$
\lambda=\frac{A T}{R_{0}^{2}}
$$

where $l, d, A$, and $T$ are the surface parameters and $R_{0}$ is an initial droplet radius. Table 4.2 lists the effective static contact angles of droplets, modeled in previous sections, with respect to $\lambda$. All angles are measured with $S E_{\bar{\theta}_{0}} \approx \pm 0.2^{\circ}$.

Figure 4.12 shows the relationship between $\lambda$ and effective static contact angles, $\theta_{\text {eff }}^{x}$ and $\theta_{\text {eff }}^{z}$ of droplets on hydrophobic and hydrophilic rough surfaces. The dependence of $\theta_{\mathrm{eff}}^{x}$ and $\theta_{\mathrm{eff}}^{z}$ on $\lambda$ is different for Wenzel and Cassie droplets. The effective contact angles of Cassie droplets decrease with increasing $\lambda$. The effective contact 
TABLE 4.2: Effective static contact angles of droplets on rough hydrophobic and hydrophilic surfaces. fr: fine roughness; mr: medium roughness; cr: coarse roughness.

\begin{tabular}{|c|c|c|c|c|c|c|c|c|c|c|c|c|}
\hline & \multicolumn{3}{|c|}{ Rectangular } & \multicolumn{3}{|c|}{$\begin{array}{c}\text { Dual- } \\
\text { rectangular }\end{array}$} & \multicolumn{3}{|c|}{ Sinusoidal } & \multicolumn{3}{|c|}{$\begin{array}{c}\text { Dual- } \\
\text { sinusoidal }\end{array}$} \\
\hline$\lambda$ & $\begin{array}{l}0.0469 \\
\text { (fr) }\end{array}$ & $\begin{array}{l}0.0781 \\
(\mathrm{mr})\end{array}$ & $\begin{array}{l}0.0977 \\
(\mathrm{cr})\end{array}$ & $\begin{array}{l}0.0469 \\
\text { (fr) }\end{array}$ & $\begin{array}{l}0.0781 \\
(\mathrm{mr})\end{array}$ & $\begin{array}{l}0.0977 \\
(\mathrm{cr})\end{array}$ & $\begin{array}{l}0.0625 \\
(\mathrm{fr})\end{array}$ & $\begin{array}{l}0.0781 \\
(\mathrm{mr})\end{array}$ & $\begin{array}{l}0.0938 \\
(\mathrm{cr})\end{array}$ & $\begin{array}{l}0.0625 \\
\text { (fr) }\end{array}$ & $\begin{array}{l}0.0781 \\
(\mathrm{mr})\end{array}$ & $\begin{array}{l}0.0938 \\
(\mathrm{cr})\end{array}$ \\
\hline \multicolumn{13}{|c|}{ Hydrophobic surfaces } \\
\hline$\theta_{\mathrm{eff}}^{x}$ & $152.9^{\circ}$ & $142.7^{\circ}$ & $130.3^{\circ}$ & $151.5^{\circ}$ & $135.5^{\circ}$ & $125.9^{\circ}$ & $144.6^{\circ}$ & $128.3^{\circ}$ & $145.3^{\circ}$ & $111.8^{\circ}$ & $121.2^{\circ}$ & $135.7^{\circ}$ \\
\hline$\theta_{\text {eff }}^{z}$ & $123.3^{\circ}$ & $124.6^{\circ}$ & $125.5^{\circ}$ & $150.6^{\circ}$ & $136.6^{\circ}$ & $128.1^{\circ}$ & $106.7^{\circ}$ & $110.5^{\circ}$ & $118.4^{\circ}$ & $110.5^{\circ}$ & $122.9^{\circ}$ & $135.7^{\circ}$ \\
\hline$\varepsilon_{\text {eff }}$ & $29.6^{\circ}$ & $18.1^{\circ}$ & $4.8^{\circ}$ & $0.9^{\circ}$ & $1.1^{\circ}$ & $2.2^{\circ}$ & $37.9^{\circ}$ & $17.8^{\circ}$ & $26.9^{\circ}$ & $1.3^{\circ}$ & $1.7^{\circ}$ & $0^{\circ}$ \\
\hline \multicolumn{13}{|c|}{ Hydrophilic surfaces } \\
\hline$\theta_{\text {eff }}^{x}$ & $103.7^{\circ}$ & $129.2^{\circ}$ & $122.2^{\circ}$ & $98.1^{\circ}$ & $94.5^{\circ}$ & $96.5^{\circ}$ & $99.8^{\circ}$ & $117.6^{\circ}$ & $123.2^{\circ}$ & $105.4^{\circ}$ & $113.7^{\circ}$ & $95.7^{\circ}$ \\
\hline$\theta_{\text {eff }}^{z}$ & $80.9^{\circ}$ & $81.1^{\circ}$ & $84.7^{\circ}$ & $95.7^{\circ}$ & $94.5^{\circ}$ & $100.6^{\circ}$ & $74.6^{\circ}$ & $72.9^{\circ}$ & $79.7^{\circ}$ & $103^{\circ}$ & $114.5^{\circ}$ & $96.5^{\circ}$ \\
\hline$\varepsilon_{\text {eff }}$ & $22.8^{\circ}$ & $48.1^{\circ}$ & $37.5^{\circ}$ & $2.4^{\circ}$ & $0^{\circ}$ & $4.1^{\circ}$ & $25.2^{\circ}$ & $44.7^{\circ}$ & $43.5^{\circ}$ & $2.4^{\circ}$ & $1.2^{\circ}$ & $0.8^{\circ}$ \\
\hline
\end{tabular}

angles of Wenzel droplets may increase or decrease with increasing $\lambda$, depending on the type of surface geometry. For example, for hydrophilic dual-rectangular surfaces, the effective contact angles do not change significantly with $\lambda$ (Fig. 4.12c), while for other types of surfaces, $\theta_{\text {eff }}$ may increase or decrease with increasing $\lambda$.

The largest effective contact angles are achieved by Cassie droplets on hydrophobic dual-rectangular surfaces, and the smallest effective contact angles are reached by Wenzel droplets on hydrophilic sinusoidal and dual-sinusoidal surfaces. The angle $\theta_{\text {eff }}^{z}$ on the rectangular hydrophobic and hydrophilic surfaces is close to the corresponding $\theta_{0}$, while $\theta_{\text {eff }}^{z}$ of sinusoidal hydrophobic and hydrophilic surfaces is smaller than $\theta_{0}$. For all other considered surfaces, $\theta_{\text {eff }}^{x}$ and $\theta_{\text {eff }}^{z}$ are larger than the corresponding $\theta_{0}$.

We quantify the directional dependence of the effective static contact angle by $\varepsilon_{\text {eff }}$, the difference between $\theta_{\text {eff }}^{x}$ and $\theta_{\text {eff }}^{z}$ of each droplet:

$$
\varepsilon_{\text {eff }}=\left|\theta_{\text {eff }}^{x}-\theta_{\text {eff }}^{z}\right| \text {. }
$$

We report $\varepsilon_{\text {eff }}$ in Table 4.2 and Fig. 4.13 for all studied values of $\lambda$. For dual-rectangular and dual-sinusoidal hydrophobic and hydrophilic surfaces $\varepsilon_{\text {eff }}$ is less than $5^{\circ}$, while for rectangular and sinusoidal hydrophobic and hydrophilic surfaces $\varepsilon_{\text {eff }}$ varies in the range from $5^{\circ}$ to $50^{\circ}$. Droplets on rectangular and sinusoidal rough surfaces are extended in the $z$ direction parallel to groves, and pinned at sharp groove edges only in the $x$ direction, so their $\theta_{\text {eff }}^{x}$ are larger than $\theta_{\text {eff }}^{z}$ and $\varepsilon_{\text {eff }}$ may achieve $50^{\circ}$. Elevated blocks on dual-rectangular and dual-sinusoidal surfaces pose an energy barrier (Gibbs; 1961; Tsoumpas et al.; 2014) hindering the extension of droplets in both directions, so that droplets are pinned in the $x$ and $z$ directions, and $\theta_{\text {eff }}^{x, z}$ is much larger than the corresponding $\theta_{0}$ on a smooth surface, while $\varepsilon_{\text {eff }}$ stays less than $5^{\circ}$.

\subsection{The effect of resolution on effective static contact angle}

To study the effect of resolution on PF-SPH solutions, we compare static contact angles of droplets on a fine dual-rectangular-type surface obtained from PF-SPH simulations with two different resolutions. In the high-resolution simulation, the 

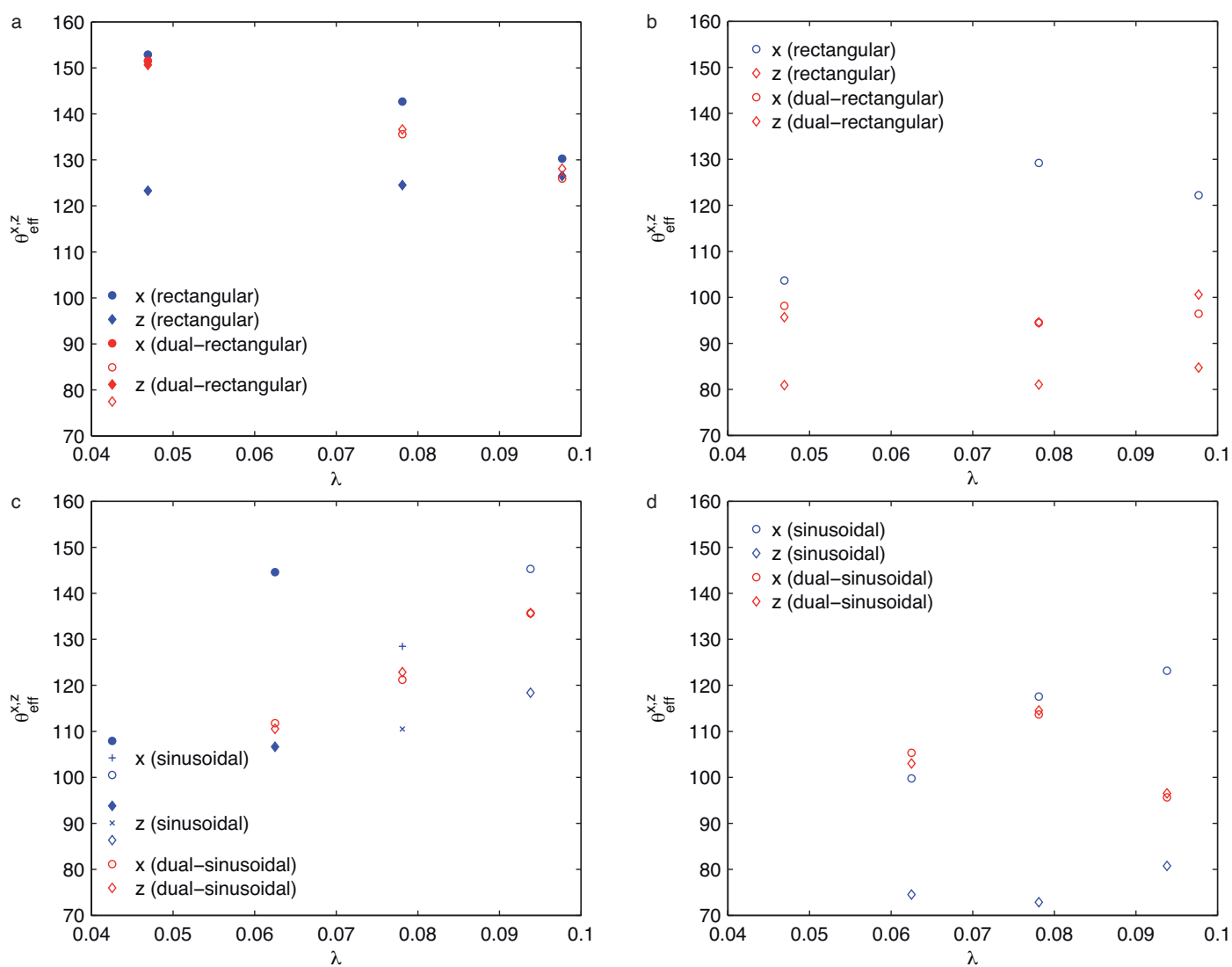

FIGURE 4.12: Effective static contact angles $\theta_{\text {eff }}^{x}$ and $\theta_{\text {eff }}^{z}$ for scaling ratio $\lambda$ between $3.5 \times 10^{-3}$ and $8.5 \times 10^{-3}$ for hydrophobic rectangular and dual-rectangular (a), hydrophilic rectangular and dualrectangular (b), hydrophobic sinusoidal and dual-sinusoidal (c), and hydrophilic sinusoidal and dual-sinusoidal (d) surfaces. Solid symbols represent droplets in a Cassie state, and open symbols represent droplets in a Wenzel state. Symbols "plus" and "cross" represent a droplet in a Cassie-Wenzel state.

number of particles is eight times higher than the number of particles in the lowresolution simulation.

The particle spacing in the high-resolution simulation is $2.5 \times 10^{-5} \mathrm{~m}$, the smoothing length is $h=8.55 \times 10^{-5} \mathrm{~m}$, the mass $m_{i}$ of each particle is $1.56 \times 10^{-11} \mathrm{~kg}$, the speed of sound $c$ is $4.5 \mathrm{~m} / \mathrm{s}$, and the surface tension of water is achieved with a fluid-fluid interaction strength $s_{\mathrm{ff}}=1.9 \times 10^{-6}$. The low-resolution simulation has the same parameters as the simulations in the preceding sections.

Figure 4.14 shows the static contact angles obtained from the simulations with these two resolutions. The angles $\theta_{\text {eff }}^{x}$ and $\theta_{\text {eff }}^{z}$ are nearly the same in both simulations.

\subsection{Transitions between Cassie and Wenzel states}

Here, we simulate ten liquid droplets with initial radii ranging from 0.5 to $1.6 \mathrm{~mm}$, which are brought into contact with fine-roughness $(d=0.2 \mathrm{~mm} ; l=0.15 \mathrm{~mm})$, medium-roughness $(d=0.25 \mathrm{~mm} ; l=0.2 \mathrm{~mm})$, and coarse-roughness $(d=0.25 \mathrm{~mm}$; $l=0.25 \mathrm{~mm}$ ) dual-rectangular hydrophobic surfaces. The effective contact angles of 


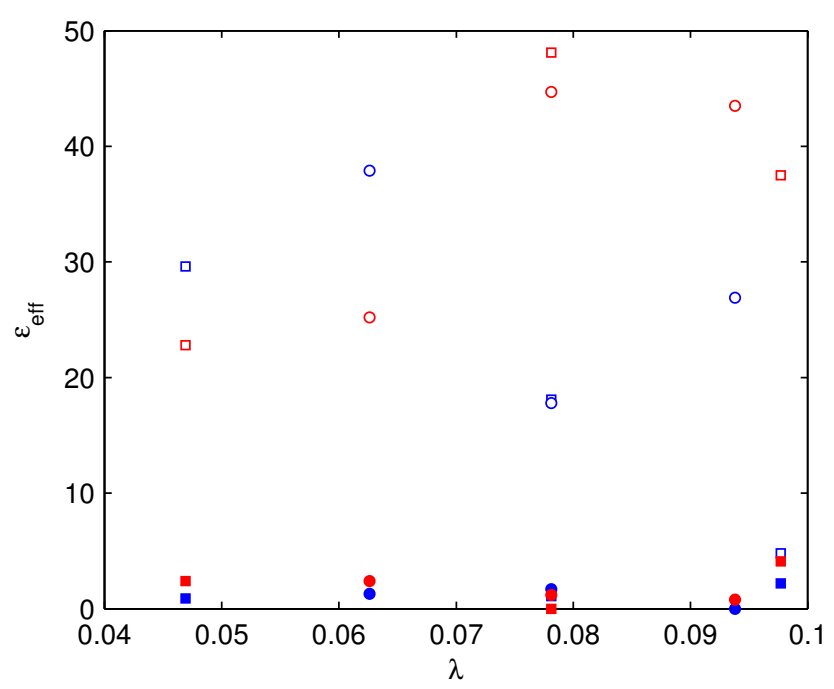

FIGURE 4.13: The effective static contact angle difference of droplets on rough hydrophobic and hydrophilic surfaces. Red: hydrophilic; blue: hydrophobic; square: rectangular surface; circle: sinusoidal surface; solid: dual surface; empty: non-dual surface.

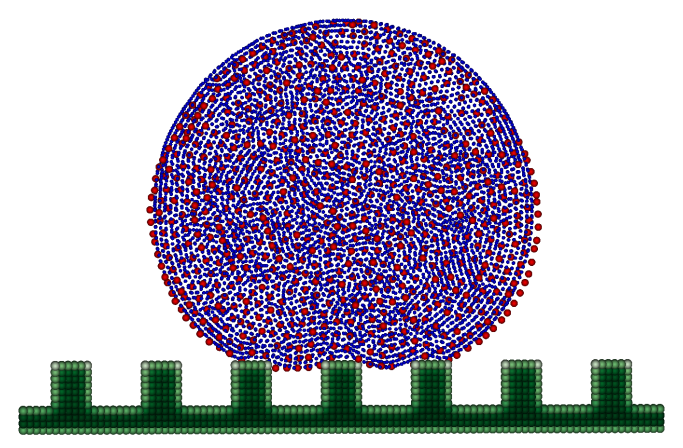

FIGURE 4.14: Comparison of effective contact angles on a fineroughness dual-rectangular surface obtained from a high-resolution (particle spacing $2.5 \times 10^{-5} \mathrm{~mm} ; \theta_{\mathrm{eff}}^{x}=149.36^{\circ} ; \theta_{\mathrm{eff}}^{z}=150.28^{\circ}$ ) and a low-resolution (particle spacing $5 \times 10^{-5} \mathrm{~mm}$; $\theta_{\mathrm{eff}}^{x}=151.52^{\circ}$; $\theta_{\text {eff }}^{z}=150.84^{\circ}$ ) simulation. Green particles: solid surface; red particles: low resolution; blue particles: high resolution.

these droplets are listed in Table 4.3. Figure 4.15 demonstrates six liquid droplets on a fine-roughness dual-rectangular surface.

All droplets on rough surfaces create unique shapes depending on the number of blocks they touch, so their $\theta_{\text {eff }}^{x, z}$ values vary in the range from $120.6^{\circ}$ to $151.5^{\circ}$, and $\varepsilon_{\text {eff }}$ varies in the range from $0.1^{\circ}$ to $14^{\circ}$. The variation in $\theta_{\text {eff }}^{x, z}$ for different droplet sizes can be explained by the Gibbs criterion (Gibbs; 1961), which attributes the pinning effects of the liquid-air interface to sharp edges of the solid surface. In our simulations, for two different-size droplets placed on equal number of blocks (droplets with $R_{\text {eq }}=1.11 \mathrm{~mm}$ and $1.3 \mathrm{~mm}$ in Fig. 4.16), the larger droplet creates larger $\theta_{\text {eff, }}^{x, z}$ because it is pinned by the edge of the block. A further increase of the droplet size (the droplet with $R_{\mathrm{eq}}=1.5 \mathrm{~mm}$ in Fig. 4.16) leads to an immediate jump to the next block and decrease of $\theta_{\text {eff }}^{x, z}$.

We observe a transition between Wenzel and Cassie regimes based on droplet 


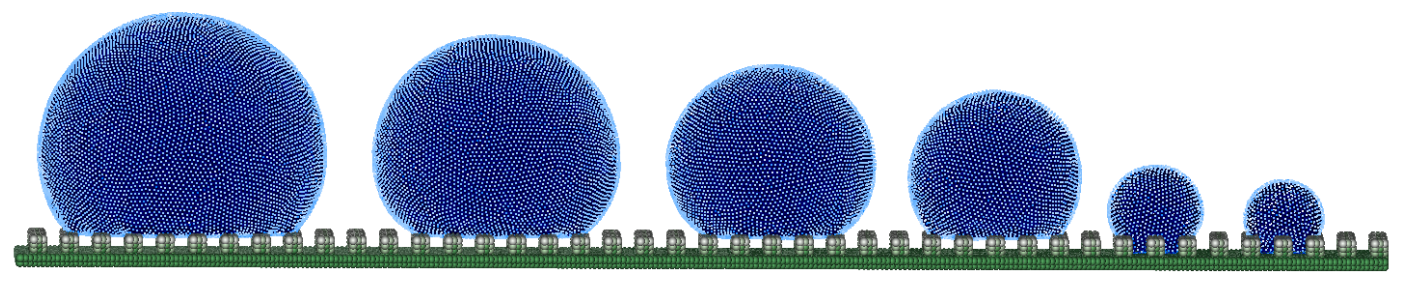

FIGURE 4.15: Droplets on a fine-roughness dual-rectangular surface. Equilibrated radii of droplets are $1.5,1.3,1.11,0.92,0.54$, and $0.44 \mathrm{~mm}$.

TABLE 4.3: Effective static contact angles of droplets with $R_{0}$ ranging from 0.5 to $1.6 \mathrm{~mm}$ on fine-, medium-, and coarse-roughness dualrectangular hydrophobic surfaces.

\begin{tabular}{|c|c|c|c|c|c|c|c|c|c|c|}
\hline$R_{0}(\mathrm{~mm})$ & 0.5 & 0.6 & 0.7 & 0.8 & 0.9 & 1.0 & 1.1 & 1.2 & 1.4 & 1.6 \\
\hline$R_{\text {eq }}(\mathrm{mm})$ & 0.44 & 0.54 & 0.64 & 0.73 & 0.83 & 0.92 & 1.02 & 1.11 & 1.3 & 1.5 \\
\hline$\Delta P(\mathrm{~Pa})$ & 330.68 & 269.33 & 227.34 & 199.32 & 175.3 & 158.15 & 142.65 & 131.08 & 111.92 & 97.00 \\
\hline \multicolumn{11}{|c|}{ Fine roughness } \\
\hline$p_{c}(\mathrm{~Pa})$ & \multicolumn{10}{|c|}{235.81} \\
\hline$\theta_{\mathrm{eff}}^{x}$ & $122.8^{\circ}$ & $135.7^{\circ}$ & $123.7^{\circ}$ & $151.5^{\circ}$ & $133.6^{\circ}$ & $139.3^{\circ}$ & $142.1^{\circ}$ & $129.8^{\circ}$ & $136.2^{\circ}$ & $126.1^{\circ}$ \\
\hline$\theta_{\text {eff }}^{\mathrm{eff}}$ & $124.1^{\circ}$ & $137.9^{\circ}$ & $122.7^{\circ}$ & $150.6^{\circ}$ & $135.2^{\circ}$ & $140.7^{\circ}$ & $143.4^{\circ}$ & $136.8^{\circ}$ & $136.7^{\circ}$ & $126.2^{\circ}$ \\
\hline$\varepsilon_{\text {eff }}$ & $1.3^{\circ}$ & $2.2^{\circ}$ & $1.0^{\circ}$ & $0.1^{\circ}$ & $1.6^{\circ}$ & $1.4^{\circ}$ & $1.3^{\circ}$ & $7.0^{\circ}$ & $0.5^{\circ}$ & $0.1^{\circ}$ \\
\hline \multicolumn{11}{|c|}{ Medium roughness } \\
\hline$p_{c}(\mathrm{~Pa})$ & \multicolumn{10}{|c|}{193.49} \\
\hline$\theta_{\mathrm{eff}}^{x}$ & $123.4^{\circ}$ & $135.2^{\circ}$ & $142.7^{\circ}$ & $135.5^{\circ}$ & $139.5^{\circ}$ & $129.5^{\circ}$ & $129.1^{\circ}$ & $137.4^{\circ}$ & $135.6^{\circ}$ & $132.8^{\circ}$ \\
\hline$\theta_{\text {eff }}^{z}$ & $124.1^{\circ}$ & $124.3^{\circ}$ & $138.4^{\circ}$ & $136.6^{\circ}$ & $131.7^{\circ}$ & $129.4^{\circ}$ & $131.2^{\circ}$ & $126.6^{\circ}$ & $137.7^{\circ}$ & $137.2^{\circ}$ \\
\hline$\varepsilon_{\mathrm{eff}}$ & $0.7^{\circ}$ & $10.9^{\circ}$ & $4.3^{\circ}$ & $1.1^{\circ}$ & $7.8^{\circ}$ & $0.1^{\circ}$ & $2.1^{\circ}$ & $0.8^{\circ}$ & $2.2^{\circ}$ & $4.4^{\circ}$ \\
\hline \multicolumn{11}{|c|}{ Coarse roughness } \\
\hline$p_{c}(\mathrm{~Pa})$ & \multicolumn{10}{|c|}{209.61} \\
\hline$\theta_{\mathrm{eff}}^{x}$ & $120.6^{\circ}$ & $125.6^{\circ}$ & $136.6^{\circ}$ & $125.9^{\circ}$ & $130.8^{\circ}$ & $140.7^{\circ}$ & $146.4^{\circ}$ & $135.2^{\circ}$ & $128.9^{\circ}$ & $135.8^{\circ}$ \\
\hline$\theta_{\mathrm{eff}}^{z}$ & $135.3^{\circ}$ & $125.0^{\circ}$ & $139.0^{\circ}$ & $128.1^{\circ}$ & $133.5^{\circ}$ & $126.7^{\circ}$ & $141.1^{\circ}$ & $130.4^{\circ}$ & $141.7^{\circ}$ & $126.6^{\circ}$ \\
\hline$\varepsilon_{\text {eff }}$ & $14.7^{\circ}$ & $0.6^{\circ}$ & $3.4^{\circ}$ & $2.2^{\circ}$ & $2.7^{\circ}$ & $14.0^{\circ}$ & $5.3^{\circ}$ & $4.8^{\circ}$ & $12.8^{\circ}$ & $9.2^{\circ}$ \\
\hline
\end{tabular}

size (Figs. 4.15 and 4.17). Droplets with $R_{\text {eq }}>0.64 \mathrm{~mm}$ on a fine-roughness dualrectangular surface remain in the Cassie regime, droplets with $R_{\mathrm{eq}} \leq 0.64 \mathrm{~mm}$ are in a Wenzel state. Various authors (e.g., Dorrer and Rühe; 2007, 2009; Zheng et al.; 2005) have investigated this transition phenomenon in terms of critical capillary pressure $p_{c}$ :

$$
p_{c}=-\frac{\sigma f \cos \left(\theta_{0}\right)}{(1-f) L}
$$

where $\sigma$ is the water surface tension, $\theta_{0}$ is the corresponding static contact angle of a droplet on a flat hydrophobic surface, and $f$ is a fraction of the wetted projection area, where $L=l / 4$ and $f=l^{2} /(l+d)^{2}$. Here $l$ and $d$ are the surface parameters as described in the previous chapters.

A Cassie-to-Wenzel transition occurs if the pressure inside the droplet, $\Delta P$, becomes larger than $p_{c}$, where $\Delta P$ can be found from the Young-Laplace law:

$$
\Delta P=\frac{2 \sigma}{R_{\mathrm{eq}}}
$$

For a fine-roughness dual-rectangular surface and the given fluid configuration, 


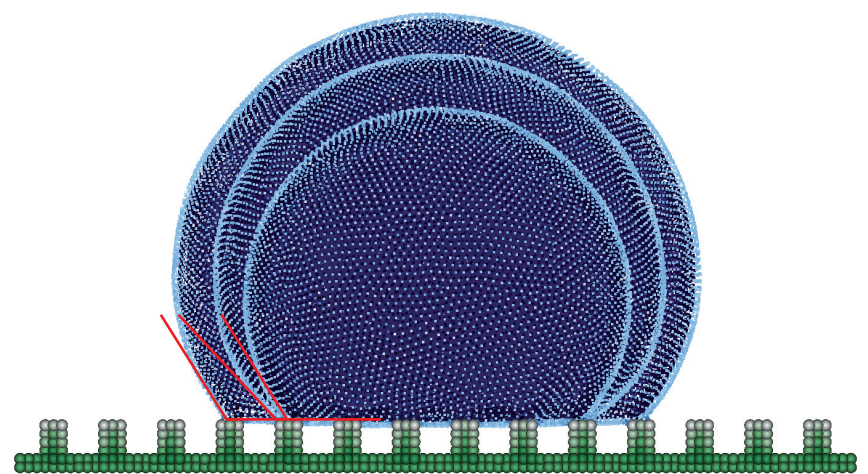

FIGURE 4.16: Pinning effect of droplets on a fine-roughness dualrectangular surface. Equilibrated radii of droplets are 1.11, 1.3, and $1.5 \mathrm{~mm}$.

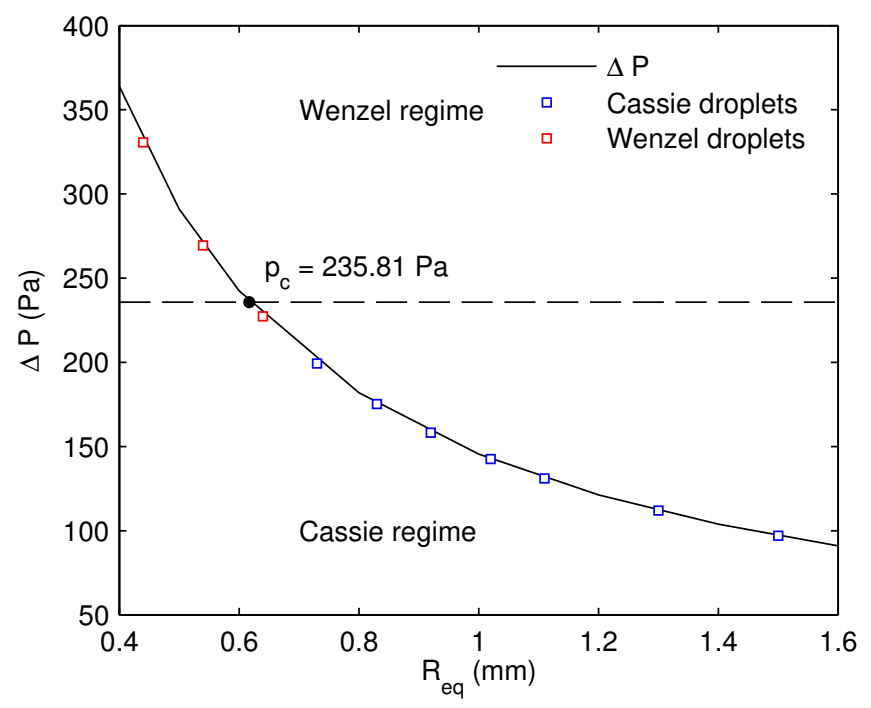

FIGURE 4.17: Cassie-to-Wenzel transition based on critical capillary pressure and internal pressures of droplets with $R_{\text {eq }}$ ranging from $0.44 \mathrm{~mm}$ to $1.5 \mathrm{~mm}$ for a fine-roughness dual-rectangular surface.

droplets switch from a Cassie to a Wenzel state when $\Delta P>p_{c}\left(p_{c}=235.81 \mathrm{~Pa}\right)$ at a radius $R_{\text {eq }}<0.62 \mathrm{~mm}$ (Fig. 4.17).

Some droplets with a value $\Delta P$ close to the theoretical value $p_{c}$ (like a droplet with $R_{\mathrm{eq}}=0.64 \mathrm{~mm}$ in Fig. 4.17) can be in both Cassie and Wenzel states, such that no clear transition point can be detected. Instead we define a transition region for droplets with $\Delta P=p_{c} \pm 30 \mathrm{~Pa}$ which can be in both states (Fig. 4.18) based on our simulation results. A region between two dashed lines in Fig. 4.18 represents the region at which the Cassie-to-Wenzel transition occurs for all types of dual rectangular surfaces. All large droplets with $R_{\mathrm{eq}} \geq 0.92 \mathrm{~mm}(\Delta P \leq 158.15 \mathrm{~Pa})$ are in a Cassie state, while small droplets with $R_{\text {eq }} \leq 0.64 \mathrm{~mm}(\Delta P \geq 227.34 \mathrm{~Pa})$ are in a Wenzel state. The width of the transition region may depend on resolution effects or pressure fluctuations during the equilibration of droplets on the surface.

Next, we investigate the dependence of droplet state on initial conditions. We simulate droplets with $R_{\mathrm{eq}}=0.64 \mathrm{~mm}$ and $1.3 \mathrm{~mm}$, which are brought into contact with a fine-roughness dual-rectangular surface. In the first case, the droplet center is located between two rectangular blocks (left droplets in Figs. 4.19a and 4.19c), for the second case, the droplet center is located above the center of a rectangular block 


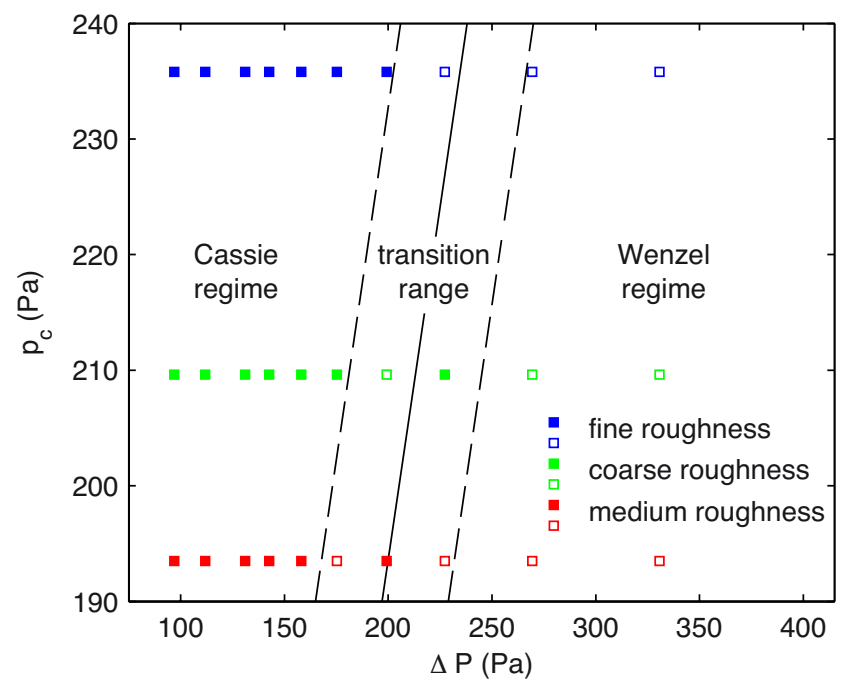

FIGURE 4.18: Cassie-to-Wenzel transition based on critical capillary pressures and internal pressures of droplets ranging from 97.00 $\mathrm{Pa}$ to $330.68 \mathrm{~Pa}$ (corresponding $R_{\mathrm{eq}}$ are ranging from $1.5 \mathrm{~mm}$ to $0.44 \mathrm{~mm}$ ) for fine-, medium-, and coarse-roughness dual-rectangular surfaces.

Open symbols: Wenzel regime; filled symbols: Cassie regime.

(right droplets in Figs. 4.19a and 4.19c). For both cases the droplet state remains the same, independent on initial placement relative to the surface roughness. The small droplet stays in a Wenzel state, and the large one in a Cassie state. This difference is caused by the initial placement of droplets relative to the roughness, which influences the effective static contact angle due to pinning effects (Table 4.4). A small droplet with a center located above a block has a larger effective contact angle. For the large droplet the effective contact angle is larger if the droplet center is located between blocks.

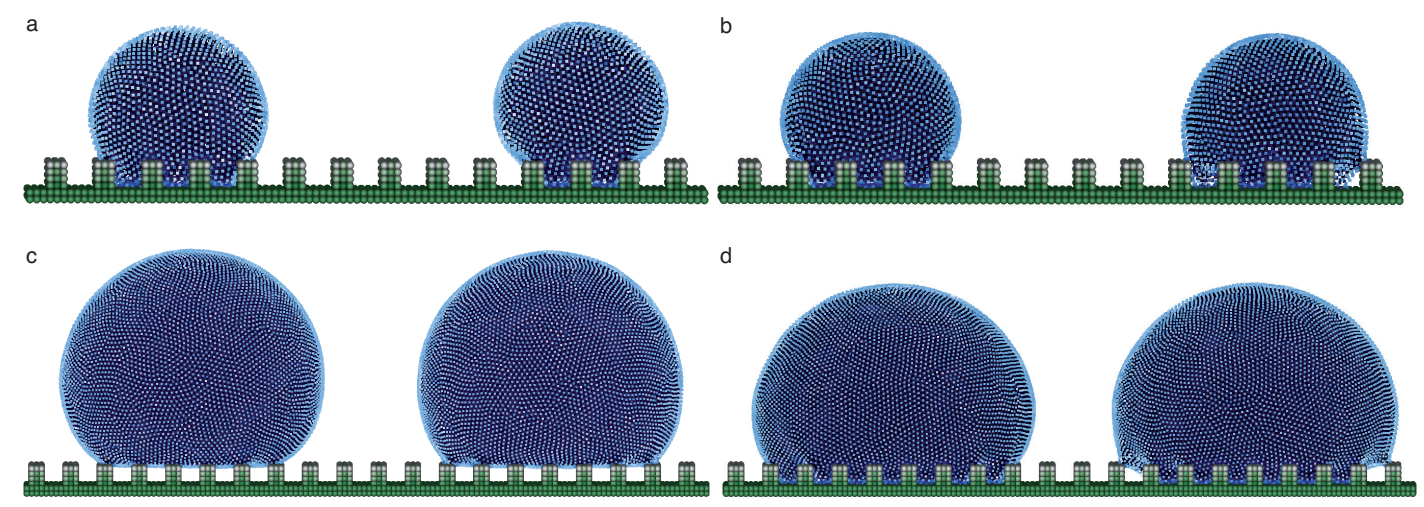

FIGURE 4.19: Droplet states depending on initial conditions: (a) droplets with $R_{\mathrm{eq}}=0.64 \mathrm{~mm}$ are brought into contact with a rough surface; (b) droplets with $R_{\text {eq }}=0.64 \mathrm{~mm}$ are dropped from $1.3 \mathrm{~mm}$ height; (c) droplets with $R_{\mathrm{eq}}=1.3 \mathrm{~mm}$ are brought into contact with a rough surface; (d) droplets with $R_{\mathrm{eq}}=1.3 \mathrm{~mm}$ are dropped from $1.75 \mathrm{~mm}$ height.

Figures $4.19 \mathrm{~b}$ and $4.19 \mathrm{~d}$ show droplets with $R_{\text {eq }}=0.64 \mathrm{~mm}$ and $1.3 \mathrm{~mm}$, which are dropped from $1.75 \mathrm{~mm}$ height (measured between surface and droplet center). Small droplets with $R_{\mathrm{eq}}=0.64 \mathrm{~mm}$ stay in a Wenzel state (Fig. 4.19b), while large droplets with $R_{\mathrm{eq}}=1.3 \mathrm{~mm}$ turn from a Cassie to Wenzel state (Figure $4.19 \mathrm{~d}$ ) due 
TABLE 4.4: Effective static contact angles of droplets with $R_{\mathrm{eq}}=$ $0.64 \mathrm{~mm}$ and $1.3 \mathrm{~mm}$ on a fine-roughness dual-rectangular hydrophobic surface depending on initial conditions.

\begin{tabular}{|c|c|c|c|c|c|c|c|c|}
\hline & \multicolumn{4}{|c|}{$R_{\mathrm{eq}}=0.64 \mathrm{~mm}$} & \multicolumn{4}{|c|}{$R_{\mathrm{eq}}=1.3 \mathrm{~mm}$} \\
\hline \multirow{2}{*}{$\begin{array}{l}\text { Mode of } \\
\text { placement }\end{array}$} & \multicolumn{2}{|c|}{ Immediate contact $^{2}$} & \multicolumn{2}{|c|}{ Dropped $^{3}$} & \multicolumn{2}{|c|}{ Immediate contact } & \multicolumn{2}{|c|}{ Dropped } \\
\hline & $\begin{array}{l}\text { Pit- } \\
\text { centered }\end{array}$ & $\begin{array}{l}\text { Block- } \\
\text { centered }\end{array}$ & $\begin{array}{l}\text { Pit- } \\
\text { centered }\end{array}$ & $\begin{array}{l}\text { Block- } \\
\text { centered }\end{array}$ & $\begin{array}{l}\text { Pit- } \\
\text { centered }\end{array}$ & $\begin{array}{l}\text { Block- } \\
\text { centered }\end{array}$ & $\begin{array}{l}\text { Pit- } \\
\text { centered }\end{array}$ & $\begin{array}{l}\text { Block- } \\
\text { centered }\end{array}$ \\
\hline$\theta_{\mathrm{eff}}^{x}$ & $121.7^{\circ}$ & $138.4^{\circ}$ & $118.7^{\circ}$ & $113.3^{\circ}$ & $137.9^{\circ}$ & $129.5^{\circ}$ & $118.8^{\circ}$ & $113.3^{\circ}$ \\
\hline$\theta_{\text {eff }}^{\text {eff }}$ & $122.1^{\circ}$ & $137.9^{\circ}$ & $119.1^{\circ}$ & $113.5^{\circ}$ & $138.2^{\circ}$ & $130.4^{\circ}$ & $118.4^{\circ}$ & $113.8^{\circ}$ \\
\hline$\varepsilon_{\text {eff }}$ & $0.4^{\circ}$ & $0.5^{\circ}$ & $0.4^{\circ}$ & $0.2^{\circ}$ & $0.3^{\circ}$ & $0.9^{\circ}$ & $0.4^{\circ}$ & $0.5^{\circ}$ \\
\hline
\end{tabular}

to additional pressure caused by gravitational impact. Therefore, the initial height from which droplets are dropped influences the effective contact angle hysteresis. Both, small and large droplets dropped from $1.75 \mathrm{~mm}$ height have smaller effective contact angles than droplets placed directly above a surface.

\subsection{Droplet flow on rough surfaces}

In this section, we study droplet flow on rough surfaces with a surface inclination angle $\alpha$ ranging from $10^{\circ}$ to $90^{\circ}$. We create two types of rough surfaces: a surface with rectangular bars oriented parallel to the flow direction and one with rectangular bars oriented perpendicular to the flow direction. For comparison, we also simulate flow on smooth surfaces. We simulate flow of two different states: flow of Cassie droplets on microscopically hydrophobic surfaces (with $\theta_{0}=122.7^{\circ}$ corresponding to $s_{\text {sf }}=0$ ), and flow of Wenzel droplets on microscopically hydrophilic surfaces (with $\theta_{0}=84.7^{\circ}$ corresponding to $s_{\mathrm{sf}}=1.3 \times 10^{-6}$ ). In these simulations, a droplet is discretized with 195216 fluid particles and the surface with approximately 235000 solid particles. The simulations are run on 32 cores.

Figure 4.20 shows simulation results for Cassie and Wenzel droplet flows on these rough surfaces with $\alpha=90^{\circ}$ after 50000 time steps $(t=46.296 \mathrm{~ms})$. Cassie and Wenzel droplets can easily slide along the rough surface with inclination angles $\alpha$ ranging from $10^{\circ}$ to $90^{\circ}$ if rectangular bars are oriented parallel to the flow direction. However, if rectangular bars are oriented perpendicular to the flow direction, a Cassie droplet barely moves and a Wenzel droplet remains stationary for all surface inclination angles $\alpha$. These results show a good qualitative agreement with experimental results of Zhang et al. (2014).

Next, we investigate the relationship between Bo and Ca numbers observed in our simulations. It was shown by Podgorski et al. (2001) that droplet dynamics on smooth surfaces follows the linear scaling law:

$$
\mathrm{Ca}=\gamma \operatorname{Bosin}(\alpha)-\Delta_{\theta},
$$

where the Ca number is defined as

$$
\mathrm{Ca}=\mu v / \sigma,
$$

and the Bo number as

$$
\mathrm{Bo}=\frac{\rho g V^{2 / 3}}{\sigma} \text {. }
$$




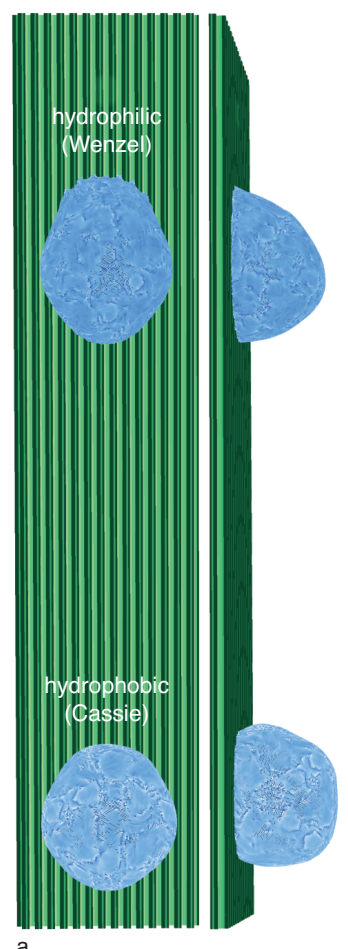

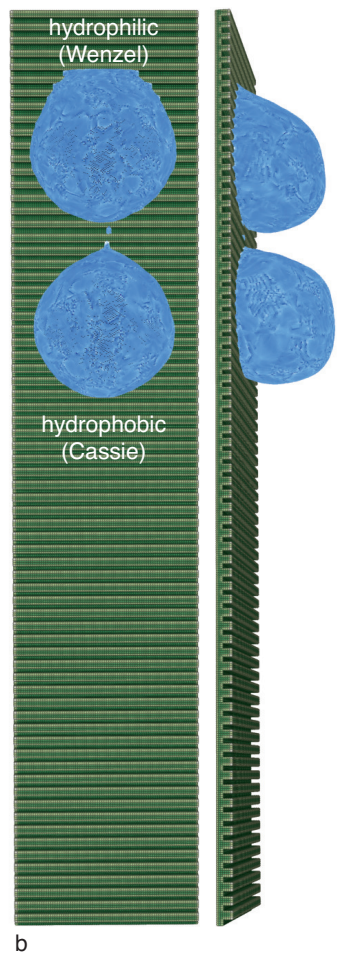

FIGURE 4.20: Hydrophobic and hydrophilic droplets flowing on a rough rectangular surface with rectangular bars oriented parallel to the flow direction (a) and rectangular bars oriented perpendicular to the flow direction (b) at the time step $t=50000$ (46.296 ms). Surface inclination angle is $\alpha=90^{\circ}$.

Here, $v$ is the droplet velocity, $V$ the equilibrium droplet volume, $\alpha$ the surface inclination angle measured from the horizontal, $\Delta_{\theta}$ is a perimeter-averaged projection factor of the surface tension, and $\gamma$ a constant related to the specific fluid-solid combination. The linear dependence between $\mathrm{Ca}$ and Bo for droplet flow on smooth surfaces was numerically confirmed by Kordilla J. (2013) via PF-SPH simulations for a range of wetting conditions; however, it has not been shown to hold for rough surfaces.

The results of our simulations, plotted in Fig. 4.21, demonstrate an existing linear relationship between $\mathrm{Ca}$ and Bo numbers for Bo $\sin \alpha<1$. For higher values of Bo $\sin \alpha$, the relationship becomes non-linear. A similar transition from linear to non-linear behavior for droplets on smooth surfaces has been reported in Kordilla J. (2013) and Podgorski et al. (2001), which is mainly caused by the deviation of droplet shapes from the spherical cap form.

Our results show that Cassie droplets on a rough surface with parallel orientation of bars to the flow direction move approximately 1.2 times faster than droplets on a smooth surface with the same $\theta_{0}$ and $\alpha$. On the other hand, Wenzel droplets on a surface with the same roughness move 1.8 times slower than a droplet on a smooth surface with the same $\theta_{0}$ and $\alpha$. Cassie droplets on the rough surface with bars perpendicular to flow do not start moving until Bo $\sin \alpha \approx 0.6$. For larger Bo $\sin \alpha$, Cassie droplets accelerate faster than droplets on a smooth surfaces with the same $\theta_{0}$. Wenzel droplets on the rough surface with "perpendicular bars" remain immobile for all considered Bo $\sin \alpha$. 


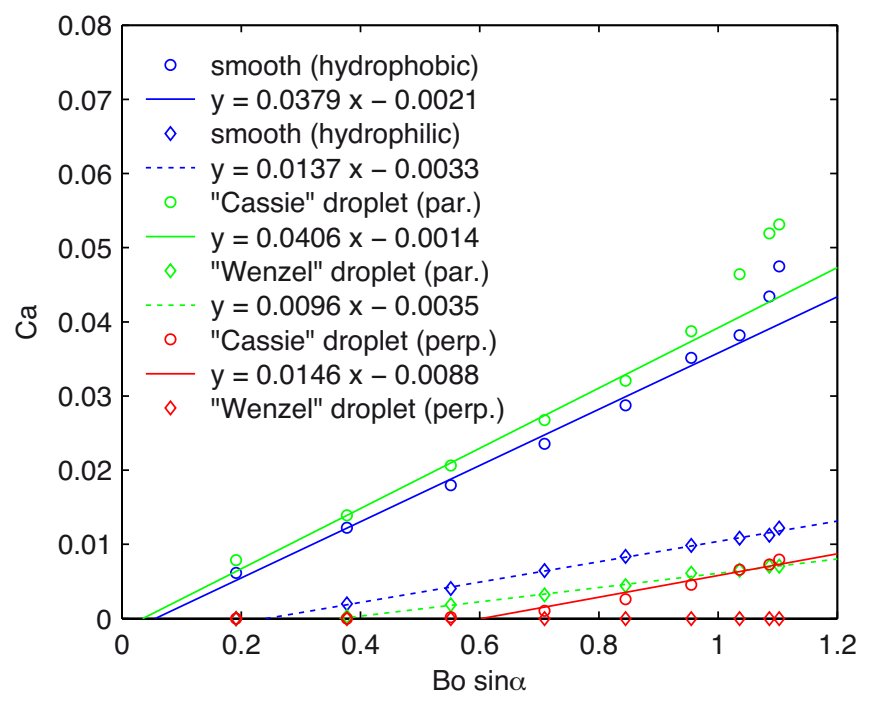

FIGURE 4.21: Dimensionless scaling for smooth and rough hydrophobic and hydrophilic surfaces with different orientations of roughness relative to the droplet flow direction. (par.) - flow parallel to the orientation of the bars, (perp.) - flow perpendicular to the orientation of bars. The surface inclination angles $\alpha$ are $10^{\circ}$, $20^{\circ}, 30^{\circ}, 40^{\circ}, 50^{\circ}, 60^{\circ}, 70^{\circ}, 80^{\circ}$ and $90^{\circ}$.

\subsection{Conclusion}

We employed a three-dimensional PF-SPH model to simulate static and dynamic droplets on rough hydrophobic and hydrophilic surfaces. We demonstrate that PF$\mathrm{SPH}$ can model ow under various wetting conditions. We also validated the model against several analytical solutions and performed a convergence study.

In PF-SPH, the surface tension and microscopic static contact angle $\theta_{0}$ result from pairwise forces added into the PF-SPH momentum conservation equation. In this work, we chose the pairwise forces to cover a wide range of microscopic static contact angles. We simulated droplets and measured effective static contact angles $\theta_{\text {eff }}$ on surfaces with different types of surface roughness, including rectangular, dualrectangular, sinusoidal, and dual-sinusoidal. For each type of surface geometry, we considered microscale hydrophobic and hydrophilic surfaces with different degrees of roughness, including fine, medium, and coarse.

We observed that Cassie droplets form on microscopically hydrophobic surfaces, and Wenzel droplets form on hydrophilic surfaces. We studied the dependence of $\theta_{\text {eff }}$ on the degree of roughness, characterized by a dimensionless ratio $\lambda$, with larger $\lambda$ corresponding to coarser surface roughness relative to the droplet size. The effective static contact angle of Cassie droplets decreases with increasing $\lambda$. The effective contact angle of Wenzel droplets may increase with increasing $\lambda$, which is attributed to the existence of pinning effects. For most studied rough surfaces, we found $\theta_{\text {eff }}$ to be greater than $\theta_{0}$. Our results showed that roughness can cause microscopically hydrophilic surfaces to behave as macroscopically hydrophobic. Moreover, microscopically hydrophilic surfaces showed even stronger macroscopic hydrophobic behavior.

In order to investigate the transition between the Cassie and Wenzel regimes we simulated liquid droplets with initial radii ranging from 0.5 to $1.6 \mathrm{~mm}$ on dualrectangular hydrophobic surfaces and compare our results to theoretical predictions. 
Good agreement is found between the analytical solution and SPH simulations. However, depending on size and internal pressure, a transition region exists where droplets may stay in a Cassie or Wenzel state. This behavior is shown to depend on initial conditions, in terms of lateral droplet position relative to the roughness and initial height. Both parameters control the hysteresis of dynamic contact angles due to pinning effects and are also present in our simulations, due to the rather coarse roughness of the surfaces relative to droplet size.

Finally, we studied droplet flow on inclined rough and smooth surfaces. We demonstrated that the type and degree of roughness, as well as the orientation of surface features relative to flow, significantly affect droplet dynamics. If rectangular bars are oriented parallel to the flow direction, water droplets can easily slide on rough hydrophobic and hydrophilic surfaces. On the other hand, if rectangular bars are oriented perpendicular to the flow direction, droplets barely move on hydrophobic surfaces and remain stationary on hydrophilic surfaces for all surface inclination angles. We demonstrated numerically that the linear scaling between the Bo and Ca numbers described in Podgorski et al. (2001) is valid not only for sliding droplets on smooth surfaces, but also for sliding droplets on rough hydrophobic and hydrophilic surfaces. The presented simulations covered a wide spectrum of wetting conditions and types of surface roughness. The influence of surface roughness and orientation on flow dynamics in the case of more complex flow regimes, such as rivulets and stable and unstable films, is part of future work.

\section{Acknowledgments.}

This work was funded by the Deutsche Forschungsgemeinschaft (DFG; German Research Foundation) under Grants No. SA 501/26-1 and No. KO 5359/1-1. E. S. was partially supported by the DAAD (German Academic Exchange Service). A.M.T. was supported by the Department of Energy (DOE)'s Office of Advanced Scientific Computing Research and Pacific Northwest National Laboratory (PNNL). PNNL is operated by Battelle for the DOE under Contract No. DE-AC05-76RL01830.

\section{Bibliography}

Allen, M. P. and Tildesley, D. J. (1989). Computer simulation of liquids, Oxford university press.

Batchelor, G. K. (1967). An introduction to fluid dynamics, Cambridge University Press, Cambridge, UK.

Bonn, D., Eggers, J., Indekeu, J., Meunier, J. and Rolley, E. (2009). Wetting and spreading, Reviews of Modern Physics 81(2): 739.

Byun, D., Kim, J., Ko, H. S. and Park, H. C. (2008). Direct measurement of slip flows in superhydrophobic microchannels with transverse grooves, Physics of Fluids (1994-present) 20(11): 113601.

Cassie, A. and Baxter, S. (1944). Wettability of porous surfaces, Transactions of the Faraday Society 40: 546-551.

Daub, C. D., Wang, J., Kudesia, S., Bratko, D. and Luzar, A. (2010). The influence of molecular-scale roughness on the surface spreading of an aqueous nanodrop, Faraday Discussions 146: 67-77.

Dorrer, C. and Rühe, J. (2007). Condensation and wetting transitions on microstructured ultrahydrophobic surfaces, Langmuir 23(7): 3820-3824. 
Dorrer, C. and Rühe, J. (2009). Some thoughts on superhydrophobic wetting, Soft Matter 5(1): 51-61.

Genzer, J. and Efimenko, K. (2006). Recent developments in superhydrophobic surfaces and their relevance to marine fouling: a review, Biofouling 22(5): 339-360.

Gibbs, J. W. (1961). The scientific papers of j. william gibbs, vol. i, III (Longmas, Green and Company, London, 1906) .

Huang, J. J., Shu, C. and Chew, Y. T. (2009). Lattice boltzmann study of droplet motion inside a grooved channel, Physics of Fluids (1994-present) 21(2): 022103.

Kordilla J., Tartakovsky A.M., G. T. (2013). A smoothed particle hydrodynamics model for droplet and film flow on smooth and rough fracture surfaces, Advances in Water Resources 59: 1-14.

Monaghan, J. J. (2005). Smoothed particle hydrodynamics, Reports on Progress in Physics 68(8).

Morris, J. P., Fox, P. J. and Zhu, Y. (1997). Modeling low reynolds number incompressible flows using sph, Journal of Computational Physics 136(1): 214-226.

Plimpton, S. (1995). Fast parallel algorithms for short-range molecular dynamics, Computational Physics 117(1): 1-19.

Podgorski, T., Flesselles, J.-M. and Limat, L. (2001). Corners, cusps, and pearls in running drops, Physical Review Letters 87(3): 036102.

Quéré, D., de Gennes, P., Brochard-Wyart, F. and Reisinger, A. (2004). Capillarity and wetting phenomena: Drops, bubbles, pearls, waves.

Stamatopoulos, C., Schutzius, T. M., Köppl, C. J., El Hayek, N., Maitra, T., Hemrle, J. and Poulikakos, D. (2016). On the shedding of impaled droplets: The role of transient intervening layers, Scientific Reports 6, 18875.

Tanner, L. (1979). The spreading of silicone oil drops on horizontal surfaces, Journal of Physics D: Applied Physics 12(9): 1473.

Tartakovsky, A. M. and Panchenko, A. (2016). Pairwise force smoothed particle hydrodynamics model for multiphase flow: surface tension and contact line dynamics, Journal of Computational Physics 305: 1119-1146.

Tartakovsky, A. and Meakin, P. (2005). Modeling of surface tension and contact angles with smoothed particle hydrodynamics, Physical Review E 72(2).

Tsoumpas, Y., Dehaeck, S., Galvagno, M., Rednikov, A., Ottevaere, H., Thiele, U. and Colinet, P. (2014). Nonequilibrium gibbs' criterion for completely wetting volatile liquids, Langmuir 30(40): 11847-11852.

Voigt, D. and Gorb, S. (2009). Egg attachment of the asparagus beetle crioceris asparagi to the crystalline waxy surface of asparagus officinalis, Proceedings of the Royal Society of London B: Biological Sciences p. rspb20091706.

Voinov, O. (1976). Hydrodynamics of wetting, Fluid Dynamics 11(5): 714-721.

Wendland, H. (1995). Piecewise polynomial, positive definite and compactly supported radial functions of minimal degree, Advances in Computational Mathematics 4(1): 389-396. 
Wenzel, R. N. (1936). Resistance of solid surfaces to wetting by water, Industrial $\mathcal{E}$ Engineering Chemistry 28(8): 988-994.

Yang, C., Tartaglino, U. and Persson, B. (2008). Nanodroplets on rough hydrophilic and hydrophobic surfaces, The European Physical Journal E 25(2): 139-152.

Zhang, P., Liu, H., Meng, J., Yang, G., Liu, X., Wang, S. and Jiang, L. (2014). Grooved organogel surfaces towards anisotropic sliding of water droplets, Advanced Materials 26(19): 3131-3135.

Zhao, Y., Lu, Q., Li, M. and Li, X. (2007). Anisotropic wetting characteristics on submicrometer-scale periodic grooved surface, Langmuir 23(11): 6212-6217.

Zheng, Q.-S., Yu, Y. and Zhao, Z.-H. (2005). Effects of hydraulic pressure on the stability and transition of wetting modes of superhydrophobic surfaces, Langmuir 21(26): 12207-12212. 



\title{
Chapter 5
}

\section{Investigation of gravity-driven infiltration instabilities in smooth and rough fractures using a pairwise-force smoothed particle hydrodynamics model ${ }^{2}$}

\begin{abstract}
.
This work investigates small-scale infiltration dynamics in smooth and rough single fractures using a three-dimensional multiphase pairwise-force smoothed particle hydrodynamics (PF-SPH) model. Gravity-driven infiltration instabilities in fractures under unsaturated conditions can significantly influence the arrival time of tracers or contaminants, and the rapid and localized recharge dynamics in fracturedporous aquifer systems. Here, we study the influence of roughness and injection rate on fluid flow modes and flow velocity. Three types of fractures are considered with different degrees of roughness, including a smooth fracture. Both the rough and smooth fractures exhibit flow instabilities, fingering, and intermittent flow regimes for low infiltration rates. In agreement with theoretical predictions, a flat fluid front is achieved when the flux $q$ supplied to a fracture is larger than the gravitationally driven saturated flux $(q>k \rho g / \mu \cos \varphi)$.

To characterize the flow instability, we calculate standard deviations of velocity along the fracture width. For the considered infiltration rates, we find that an increase in roughness decreases the flow velocity and increases the standard deviation of velocity. This is caused by a higher likelihood of flow discontinuities in the form of fingering and/or snapping rivulets. To validate our unsaturated flow simulations in fractures, we estimate the scaling of specific discharge with normalized finger velocity and compute the relationship between fingertip length and scaled finger velocity, and find a good agreement with experimental results.

- We study infiltration dynamics in smooth and rough fractures using a 3D SPH model

- Roughness induces the formation of flow fingering and preferential flow paths

- Fractal roughness leads to a deceleration of average fingertip velocities
\end{abstract}

\footnotetext{
${ }^{2}$ Shigorina, E., Kordilla, J., and Tartakovsky, A. M. (2019): Investigation of gravity-driven infiltration instabilities in smooth and rough fractures using a pairwise-force smoothed particle hydrodynamics model. Vadoze Zone Journal, 18:180159. doi:10.2136/vzj2018.08.0159.
} 
- The velocity variance of fingers positively correlates with the degree of roughness

- Normalized finger velocity and length scale in accordance with analytical predictions

\subsection{Introduction}

Preferential flows within the unsaturated zone have a significant influence on groundwater recharge, infiltration, and contaminant transport (Nimmo; 2010, 2012). In contrast to diffuse Richards-type flows commonly encountered in soils and porous media, preferential flows are usually (but not exclusively) observed in fractured-porous media and are characterized by a non-uniform water distribution within individual fractures (Cueto-Felgueroso and Juanes; 2008; Dippenaar and Van Rooy; 2016; Kordilla et al.; 2017; Nicholl and Glass; 2005; Wang et al.; 2003).

Preferential flows affect various subsurface flow processes, such as water supply and nuclear waste storage (Evans and Rasmussen; 1991), infiltration of water in karst aquifers (Geyer et al.; 2008), and can be relevant on different scales, ranging from macropores to catchment-scale fault zones (Hendrickx and Flury; 2001). On large scales hydraulic input signals are often dominated by percolating fracture networks (DiCarlo et al.; 1999; Khamforoush et al.; 2008; Mourzenko et al.; 2004; Patriarche et al.; 2007), which may provide rapid transmission through several hundreds of meters within the vadose zone.

Despite ongoing research, unsaturated flows in fractures are not well understood due to various rate-dependent fracture-specific flow regimes, scale effects, characterization of process parameters across scales, and the assessment of their relevance in the prediction of large-scale problems, e.g., the regional hydraulics of fault zones (Eker and Akin; 2006; Hendrickx and Flury; 2001).

Most numerical large-scale studies employ the Richards' equation (Richards; 1931) to describe partially saturated flow in fractured media, which is treated as a porous continuum (Heilweil et al.; 2015; Therrien and Sudicky; 1996a). In the presence of a porous matrix, multi-continuum approaches can be used to resolve the large ratio of fractures and porous matrix permeabilities (Kordilla et al.; 2012; Wang and Narasimhan; 1985; Wu et al.; 2004).

In fully saturated systems, the parallel plate model (Dershowitz and Einstein; 1988; Snow; 1996) allows for a better discrete representation of internal fracture flow dynamics, which are subject to ongoing modifications to account for roughness effects (e.g. Wang et al.; 2015).

However, in partially saturated fractures, the distribution of water within the fracture depends on matric potential (of the adjacent porous medium), local aperture, and flow rate dependent flow modes (droplets/slugs, rivulets, films), which influence the formation of instabilities (fingering) and hence the travel times. Furthermore, the fracture-specific water distribution to a large degree controls the interfracture partitioning and redistribution dynamics (Jones et al.; 2018; Kordilla et al.; 2017; Noffz et al.; 2018), which is of importance for the application in discrete fracture network models (DFN, Cacas et al.; 1990; Hyman et al.; 2015). The complexity of unsaturated flows and the existence of several highly dynamic flow regimes in fractures has been demonstrated by various authors (Ghezzehei; 2004; Tokunaga and Wan; 1997, 2001). Depending on infiltration rates, flow regimes switch from adsorbed films (with average flow velocities on the order of $10^{-7} \mathrm{~m} \cdot \mathrm{s}^{-1}$ ) to droplet 
flows and finally rivulet flows at high infiltration rates. Finally, at very high flow rates, rivulets merge, and continuous (wavy) films can occur.

Transitions between flow modes and, therefore, the instability of an injected fluid front depend on the complex force balance between gravity, capillary, and viscous forces. Even under idealized conditions, e.g., smooth fracture surfaces and constant infiltration rates, fluid fronts in fractures are prone to develop instabilities, i.e., fracture-specific preferential flow paths, due to the strong impact of gravitational forces and relatively low capillary action (Nicholl et al.; 1994). The number of these discontinuities (rivulets and/or droplet streams) is generally larger in rough fractures (Briggs et al.; 2017; Wang et al.; 2016). Injected fluid follows the paths of least resistance, accumulates in depressions, or flows around elevated parts of the rough surface to form fracture-specific preferential flow pathways.

Laboratory experiments can provide valuable insights into infiltration dynamics in unsaturated fractures. Most laboratory setups investigating unsaturated flows consist of two (textured) parallel glass plates (Hele-Shaw cell), separated by a small aperture, representing a fracture (Jones et al.; 2018; Nicholl and Glass; 2005; Nicholl et al.; 1999). This allows to visually observe the internal transient flow dynamics, i.e., the propagation of the fluid. Laboratory experiments in natural fractures are challenging. For example, flow in a natural fracture was considered by Nicholl et al. (1994), where only the post-infiltration state could be analyzed after the setup was disassembled. Laboratory studies of saturated flow have for example been carried out by Li et al. (2018) who used cement casts to create surfaces based on fractal roughness and obtained information about flow channeling effects by measuring outflow rates along the fracture width. Brown et al. (1998) used rubber molds to manufacture transparent epoxy replicas of natural fractures and measured flow velocities using nuclear magnetic resonance imaging. They found that flow velocities in the fracture plane may vary over several orders of magnitude and maximum velocities may be higher than the mean flow velocity by a factor of 5 .

Of direct relevance to our present work are the studies of Nicholl et al. (1994) and Nicholl and Glass (2005) who experimentally studied the finger formation across a wide range of flow rates and fracture inclinations as a function of the gravitational and viscous pressure differentials along infiltrating fingers.

In this work, we investigate the development of fluid front instability and formation of complex flow modes in smooth and rough individual fractures by employing an efficient three-dimensional parallelized pairwise-force smoothed particle hydrodynamics (PF-SPH) model implemented in LAMMPS (Kordilla et al.; 2017; Plimpton; 1995). The PF-SPH-LAMMPS code has been extensively validated (Kordilla et al.; 2017; Kordilla J.; 2013; Shigorina et al.; 2017) and employed to simulate highly intermittent, gravity-driven free-surface flows under dynamic wetting conditions. Our recent work (Kordilla J.; 2013; Shigorina et al.; 2017) investigated the effect of surface roughness on droplet and rivulet flows. Here, we study the effect of roughness on average flow velocity and fluid flow profile in fractures.

We use the PF-SPH model to characterize fluid front instabilities in smooth and rough fractures for various infiltration rates in terms of finger front velocity. We observe transitions between flow modes and fluid flow profiles for different infiltration rates. Flow in fractures with two types of surface roughness are studied and compared to flow dynamics in a smooth fracture. For each infiltration rate, the average flow velocity is measured. We use the standard deviation of flow velocity to quantify flow instability or degree of dispersion, and compare the standard deviation for different infiltration rates and degrees of roughness. Finally, we study the 
specific discharge scaling with the normalized finger velocity and the relation between fingertip length and scaled finger velocity and find good agreement with the experimental results of Nicholl and Glass (2005).

\subsection{Governing equations and the PF-SPH method}

We assume that free-surface flow of water in a fracture can be described by the continuity equation,

$$
\frac{d \rho}{d t}=-\rho(\nabla \cdot \mathbf{v}), \quad \mathbf{x} \in \Omega_{f}(t),
$$

and the momentum conservation equation,

$$
\frac{d \mathbf{v}}{d t}=-\frac{1}{\rho} \nabla P+\frac{\mu}{\rho} \nabla^{2} \mathbf{v}+\mathbf{g}, \quad \mathbf{x} \in \Omega_{f}(t)
$$

subject to the Young-Laplace boundary condition

$$
P \mathbf{n}=\boldsymbol{\tau}_{w} \cdot \mathbf{n}+S \sigma \mathbf{n}, \quad \mathbf{x} \in \partial \Omega_{w a},
$$

and the continuity condition at the water-air interface $\partial \Omega_{w a}$,

$$
\left(\mathbf{v}-\mathbf{v}_{b}\right) \cdot \mathbf{n}=0, \quad \mathbf{x} \in \partial \Omega_{w a} .
$$

The contact angle is prescribed at the water-air-solid contact line $S$ and the noslip boundary condition at the boundary between water and solid phases. Here, $\boldsymbol{\tau}_{w}=\left[\mu\left(\nabla \mathbf{v}+\nabla \mathbf{v}^{\mathrm{T}}\right)\right]$ is the viscous stress tensor, $\mathbf{v}$ the fluid velocity, $\mathbf{v}_{b}$ is the boundary velocity, $P$ is the pressure, $\mu$ the viscosity, $\mathbf{n}$ is the normal vector pointing away from the non-wetting phase, and $\mathbf{g}$ the gravitational acceleration.

To numerically solve the governing equations, we extend the domain $\Omega_{f}(t)$ occupied by water to include the solid phase (walls of the fractures) as $\Omega(t)=\Omega_{f}(t) \cup$ $\Omega_{s}$, where $\Omega_{s}$ is the extension of $\Omega_{f}(t)$. Next, we discretize $\Omega_{f}(t)$ with "fluid" particles with positions denoted by $\mathbf{r}_{i} \in \Omega_{f}(t)$ and $\Omega_{s}$ with solid particles with positions $\mathbf{r}_{i} \in \Omega_{s}$. The positions of solid particles are fixed and their velocities are set to zero. The positions and velocities of fluid particles are found from the momentum conservation equation discretized with the weakly compressible pairwise SPH scheme (Kordilla et al.; 2017; Kordilla J.; 2013; Morris et al.; 1997; Tartakovsky and Meakin; 2005a):

$$
\begin{aligned}
& \frac{d \mathbf{v}_{i}}{d t}=-\sum_{j=1}^{N} m_{j}\left(\frac{P_{j}}{\rho_{j}^{2}}+\frac{P_{i}}{\rho_{i}^{2}}\right) \frac{\mathbf{r}_{i j}}{r_{i j}} \cdot \frac{d W\left(r_{i j}, h\right)}{d r_{i j}}+ \\
& 2 \mu \sum_{j=1}^{N} m_{j} \frac{\mathbf{v}_{i j}}{\rho_{i} \rho_{j} r_{i j}} \cdot \frac{d W\left(r_{i j}, h\right)}{d r_{i j}}+\mathbf{g}+\frac{1}{m_{i}} \sum_{j=1}^{N} \mathbf{F}_{i j}, \quad \mathbf{r}_{i} \in \Omega_{f}(t),
\end{aligned}
$$

and

$$
\frac{d \mathbf{r}_{i}}{d t}=\mathbf{v}_{i}, \quad \mathbf{r}_{i} \in \Omega_{f}(t),
$$

where the summation is performed over all particles including fluid and solid particles. Here, $\mathbf{r}_{i j}=\mathbf{r}_{i}-\mathbf{r}_{j}$ and $r_{i j}=\left|\mathbf{r}_{i}-\mathbf{r}_{j}\right|, m_{j}=m_{0}$ is the (constant) mass of particle $j, \rho_{j}$ and $P_{j}$ are the density and pressure of fluid carried by particle $j$, and $h$ is the support range of the kernel $W$. Fluid and solid particles are assumed to have the same mass and $\rho_{i}$ is computed for both fluid and solid particles as (Morris et al.; 
1997; Tartakovsky and Meakin; 2005a)

$$
\rho_{i}=\sum_{j=1}^{N} m_{j} W\left(\mathbf{r}_{i j}, h\right), \quad \mathbf{r}_{i} \in \Omega_{f}(t) \cup \Omega_{s}
$$

This expression conserves mass exactly and, therefore, can be used instead of the mass conservation (continuity) equation (6.3). The pressure of both fluid and solid particles is computed from the equations of state Batchelor (1967):

$$
P_{i}=P_{0}\left\{\left(\frac{\rho_{i}}{\rho_{0}}\right)^{\gamma}-1\right\}, \quad \mathbf{r}_{i} \in \Omega_{f}(t) \cup \Omega_{s} .
$$

where

$$
P_{0}=\frac{c^{2} \rho_{0}}{\gamma}
$$

$\gamma=7, \rho_{0}$ is the equilibrium particle density, and the speed of sound $c$ is chosen such that the relative density fluctuation $|\delta \rho| / \rho$ is small (less than $3 \%$ ) to approximate an incompressible fluid.

The weighting function $W$ is modeled with the third-order Wendland function (Wendland; 1995):

$$
W\left(r_{i j}, h\right)=\alpha_{k}\left\{\begin{array}{ll}
\left(1-\frac{\mathbf{r}_{i j}}{h}\right)^{4}\left(4 \frac{\mathbf{r}_{i j}}{h}+1\right) & \text { if } 0 \leq r_{i j}<h \\
0 & \text { if } r_{i j} \geq h
\end{array},\right.
$$

where $\alpha_{k}=21 /\left(2 \pi h^{3}\right)$.

The force $\mathbf{F}_{i j}$ in Eq. (6.7) is used to impose the Young-Laplace boundary condition (Tartakovsky and Panchenko; 2016). Following Tartakovsky and Meakin (2005a), Tartakovsky and Panchenko (2016), Kordilla J. (2013), we employ a combination of kernel functions to generate a continuous function with short-range repulsive and long-range attractive components:

$$
\mathbf{F}_{i j}=s_{i j} \begin{cases}\left(\tilde{A} \tilde{W}\left(r_{i j}, h_{1}\right) \frac{\mathbf{r}_{i j}}{r_{i j}}+\tilde{B} \tilde{W}\left(r_{i j}, h_{2}\right) \frac{\mathbf{r}_{i j}}{r_{i j}}\right) & \text { if } \mathbf{r}_{i j} \leq h \\ 0 & \text { if } \mathbf{r}_{i j}>h,\end{cases}
$$

where $\tilde{W}$ is the cubic spline function

$$
\tilde{W}\left(r_{i j}, h\right)= \begin{cases}1-\frac{3}{2}\left(\frac{\mathbf{r}_{i j}}{h}\right)^{2}+\frac{3}{4}\left(\frac{\mathbf{r}_{i j}}{h}\right)^{3} & \text { if } 0 \leq \frac{\mathbf{r}}{h}<0.5 \\ \frac{1}{4}\left(2-\frac{\mathbf{r}_{i j}}{h}\right)^{3} & \text { if } 0.5 \leq \frac{\mathbf{r}_{i j}}{h}<1 \\ 0 & \text { if } \frac{\mathbf{r}_{i j}}{h} \leq 1 .\end{cases}
$$

Here, $\tilde{A}, \tilde{B}, h_{1}$, and $h_{2}$ determine the shape of $\mathbf{F}_{i j}$. We set $\tilde{A}=8, \tilde{B}=-1$, and $h_{1}=0.5$, $h_{2}=1$. For a given $\mathbf{F}_{i j}$ shape, $s_{i j}$ determines the magnitude of surface tension and static contact angle.

The parameter $s_{i j}$ is equal to $s_{f f}$ for the interaction between two fluid particles and $s_{s f}$ for the interaction between fluid and solid particles. The ratio of $s_{f f}$ and $s_{s f}$ controls the static and dynamic contact angles. For a liquid to wet the surface, $s_{f f}$ should be set greater than $s_{s f}$ and vice versa. 
In SPH, the no-slip boundary condition at the fluid-solid boundary can be imposed by using ghost particles that mirror fluid particles in the direction normal to the nominal solid interface (Libersky et al.; 1993) or uniformly distributed particles in the solid phase (i.e., in the fracture wall, Morris et al.; 1997; Zhu et al.; 1999). These methods require determining the ratio of normal distances from fluid and mirror particles to the nominal solid boundary (the proximity ratio), which becomes challenging for highly irregular surfaces. Moreover, the computational costs of these methods are high compared to simpler bounce-back conditions, as demonstrated by Tartakovsky and Meakin (2006), Tartakovsky et al. (2009), and Kordilla J. (2013), and Kordilla et al. (2017).

To enforce the no-slip boundary condition at the nominal fluid-solid interface, we use the proximity ratio (Holmes et al.; 2011). We first define the state of a particle, i.e., fluid or solid with $\kappa$ and $\eta$. Next, a phase-specific number density $n_{i}^{s}$ is calculated to easily determine the solid-fluid boundary even for highly irregular (rough) surfaces,

$$
n_{i, \kappa}^{s}=\sum_{j=1}^{N} \delta_{\kappa \eta} W\left(\mathbf{r}_{i, \kappa}-\mathbf{r}_{j, \eta}, h\right),
$$

where the Kronecker delta is

$$
\delta_{\kappa \eta}= \begin{cases}1 & \kappa=\eta \\ 0 & \kappa \neq \eta\end{cases}
$$

The proximity ratio for a fluid particle can then be obtained as

$$
\phi_{i}=\frac{n_{i}^{s}}{n_{i}}
$$

where $n_{i}$ is the (total) particle density $n_{i}=\rho_{i} / m_{i}$ defined as:

$$
n_{i}=\sum_{j=1}^{N} W\left(\mathbf{r}_{i}-\mathbf{r}_{j}, h\right) .
$$

The value of $\phi$ varies from 1 for particles in the fluid phase at the distance greater than $h$ from the fluid-solid interface to 0.5 at the interface to zero for particles in the solid phase at the normal distance greater than $h$. Note that for $m_{i}=m_{0}$, the total particle number density can be found as $n_{i}=\rho_{i} / m_{i}$. To enforce a no-slip boundary condition, we return fluid particles along the normal back into the flow domain once they penetrate the boundary, i.e., for $\phi_{i}<0.5$. A smoothed color function $c_{i}$ is employed to obtain the normals:

$$
c_{i}=\sum_{j \in \Omega_{f}} \frac{m_{j}}{\rho_{j}} W\left(\mathbf{r}_{i j}, h\right),
$$

where the above summation is only over fluid particles. The surface normals can then be calculated from the gradient:

$$
\hat{\mathbf{s}}_{i}=\nabla c_{i}
$$


Penetrating particles have their velocities inverted and are returned along the normal direction by a distance $d$ proportional to the proximity ratio:

$$
d=\omega \Delta x\left(1-\frac{\phi_{i}}{0.5}\right)
$$

In this work, we set $\omega=1$, which we found to prevent particle penetration even for very complex surface geometries. In most cases, the pressure gradient and interaction force are sufficient to prevent the penetration of fluid particles in the solid phase.

Eq. (6.7) is integrated with a modified velocity-Verlet time-stepping scheme (Ganzenmüller et al.; 2011):

$$
\begin{aligned}
\mathbf{v}_{i}\left(t+\frac{1}{2} \Delta t\right) & =\mathbf{v}_{i}+\frac{1}{2} \mathbf{a}_{i}(t), \\
\overline{\mathbf{v}}_{i}(t+\Delta t) & =\mathbf{v}_{i}(t)+\Delta t \mathbf{a}_{i}, \\
\mathbf{r}_{i}(t+\Delta t) & =\mathbf{r}_{i}(t)+\Delta t \mathbf{v}_{i}\left(t+\frac{1}{2} \Delta t\right) \\
\mathbf{v}_{i}(t+\Delta t) & =\mathbf{v}_{i}\left(t+\frac{1}{2} \Delta t\right)+\frac{1}{2} \mathbf{a}_{i}(t+\Delta t) .
\end{aligned}
$$

Once new positions $\mathbf{r}_{i}(t+\Delta t)$ are calculated, the new particle acceleration $\mathbf{a}_{i}(t+$ $\Delta t)$ can be obtained using an extrapolated velocity $\overline{\mathbf{v}}_{\mathbf{i}}$. To ensure stability of the numerical solution, the following time step constraints are satisfied (Tartakovsky and Meakin; 2005a):

$$
\begin{aligned}
& \Delta t \leq 0.25 h / 3 c \\
& \Delta t \leq 0.25 \min \left(h / 3\left|\mathbf{a}_{i}\right|\right)^{1 / 2} \\
& \Delta t \leq \min \left(\rho_{i} h^{2} / 9 \mu_{i}\right),
\end{aligned}
$$

with $\left|\mathbf{a}_{i}\right|$ representing the magnitude of acceleration $\mathbf{a}_{i}$.

\subsection{Gravity-driven flow instability in initially dry fractures}

Infiltration into a fracture is controlled by gravity, viscous, and capillary forces. When gravitational forces dominate, the flow becomes unstable, and fingering occurs. Instability of the gravity-driven fluid front occurs when the flux $q$ supplied to a fracture is less than the gravitationally driven saturated flux $K_{s} \cos \varphi$ (Nicholl et al.; 1994):

$$
q<K_{s} \cos \varphi,
$$

where $K_{s}$ is the saturated hydraulic conductivity

$$
K_{s}=k \rho g / \mu \text {, }
$$

and $k$ represents the intrinsic permeability of the fracture and $\varphi$ is the fracture inclination angle measured from the vertical direction.

In this work, we numerically study the propagation of a fluid front in smooth and rough fracture planes along with the dependence on the supplied flux. Specifically, we investigate the dynamic switching of flow regimes between droplets, rivulets, and films and how it controls the formation of instabilities and average arrival times. 


\subsubsection{Flow in smooth vertical fractures}

In the following, we model flow between two smooth parallel vertical surfaces with dimensions $10 \times 30 \mathrm{~cm}$ separated by a $2.0 \mathrm{~mm}$ gap (Fig. 5.1, left). Fluid particles are assigned a density and viscosity of water $\rho_{0}=1000 \mathrm{~kg} / \mathrm{m}^{3}$ and $\mu=1.296 \times 10^{-3} \mathrm{~Pa} \mathrm{~s}$, respectively. The equilibrium density of solid and fluid particles is set to $1000 \mathrm{~kg} / \mathrm{m}^{3}$. Initially, the SPH particles (solid and fluid) are placed on a uniform cubic lattice with spacing $\Delta x=2 \times 10^{-4} \mathrm{~m}$, which results in a fluid particle mass of $m_{0}=\rho_{0} \Delta x^{3}=$ $8 \times 10^{-9} \mathrm{~kg}$. The mass of individual solid particles is set to $m_{0}$. The speed of sound is $c=2.5 \mathrm{~m} / \mathrm{s}$, the gravitational acceleration $\mathbf{g}=9.81 \mathrm{~m} / \mathrm{s}^{2}$, and the smoothing length is set to $h=n_{d}^{1 / 3} \Delta x=6.84 \times 10^{-4} \mathrm{~m}$. Here, $n_{d}=40$ is the number of particles within a volume $h^{3}$, which was found to be sufficient for three-dimensional simulations including the effect of surface tension. The fluid is injected at the fracture top with constant volumetric flux $Q$. According to Eq. (5.22), for a stable fluid front to develop, the flux must be $Q \geq 5.06 \times 10^{-4} \mathrm{~m}^{3} / \mathrm{s}(q \geq 2.52 \mathrm{~m} / \mathrm{s})$. To investigate the fluid front instability, we perform simulations with $Q$ varying from $Q=$ $4 \times 10^{-6} \mathrm{~m}^{3} / \mathrm{s}$ to $8 \times 10^{-4} \mathrm{~m}^{3} / \mathrm{s}$ (Fig. 5.2 ). For this simulation setup, the fracture is discretized with 2250000 solid particles, and the fluid is discretized with 670000 to 14900000 particles, depending on the infiltration rate and duration. Simulations are run on 64 and 128 processors.
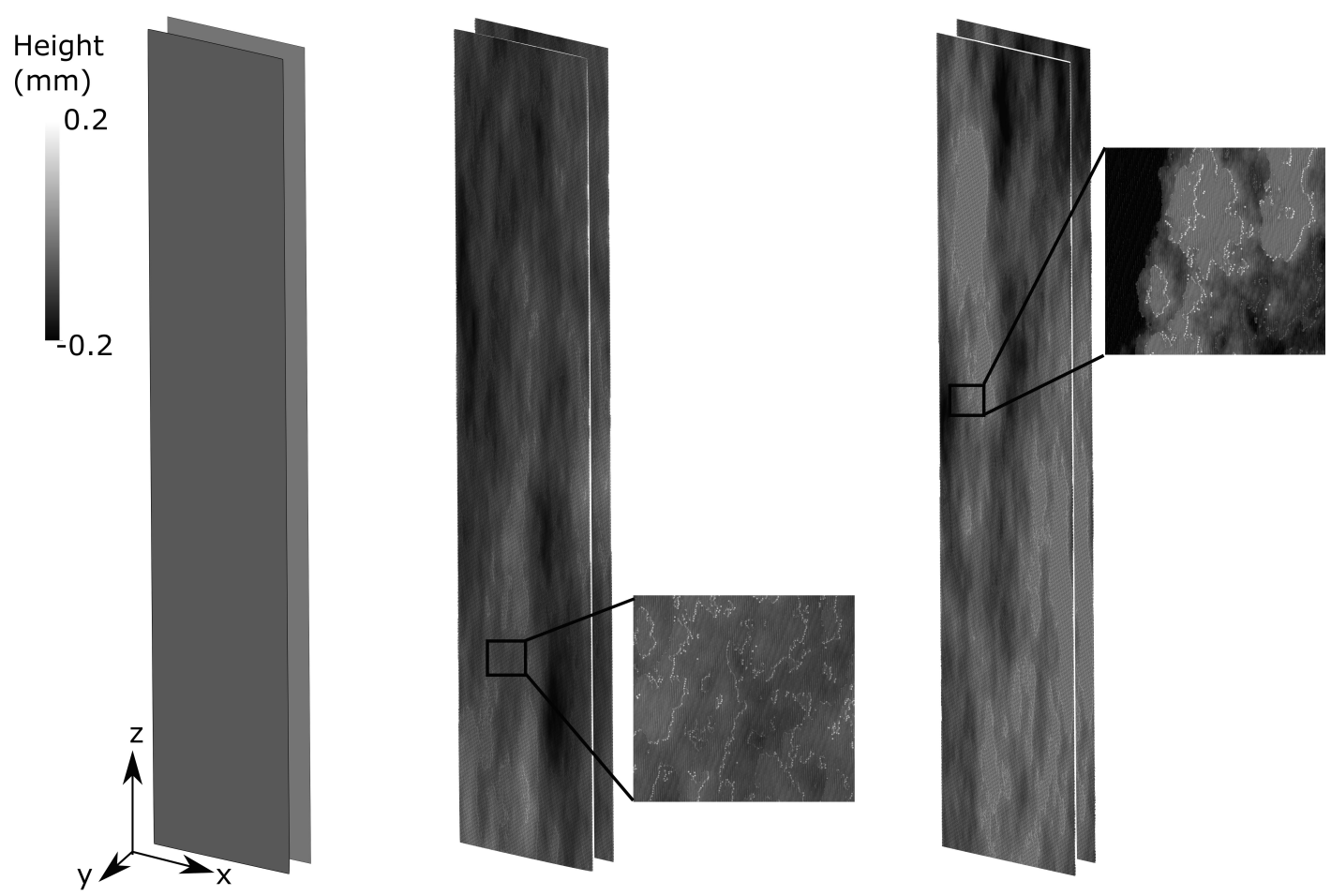

FIGURE 5.1: Fracture configurations: smooth (left); rough with $\zeta=$ 0.75 and $\Delta=10 \mathrm{~mm}$ (middle); rough with $\zeta=0.75$ and $\Delta=20 \mathrm{~mm}$ (right).

To measure the average flow velocity, we divide the fracture surface into $n=20$ longitudinal sections of equal width. Each section has a width of $0.5 \mathrm{~cm}$ and length of $30 \mathrm{~cm}$. At every time step, we measure the fluid front propagation within each 
section. The fluid velocity for each section $v_{s e c}^{i}$ is found as

$$
v_{s e c}^{i}=\frac{z(t+\Delta t)-z(t)}{\Delta t},
$$

where $z(t+\Delta t)$ and $z(t)$ are the maximum fluid front positions into the direction of flow at time step $(t+\Delta t)$ and $t$, respectively. The average flow velocity $\bar{v}$ for the whole fracture is then found as an arithmetic mean of flow velocities within each section:

$$
\bar{v}=\frac{1}{20} \sum_{i=1}^{20} v_{s e c}^{i}
$$

The velocities $\bar{v}$ for different $Q$ are listed in Table 5.1. The average velocity $\bar{v}^{s}$ increases with increasing $Q$ (Fig. 5.5, squares).

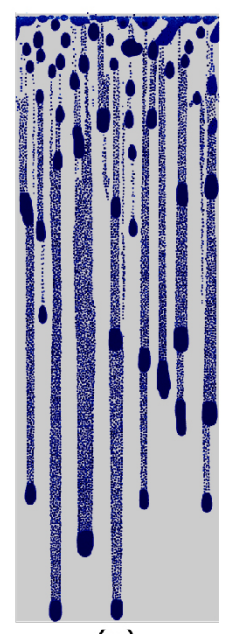

(a)

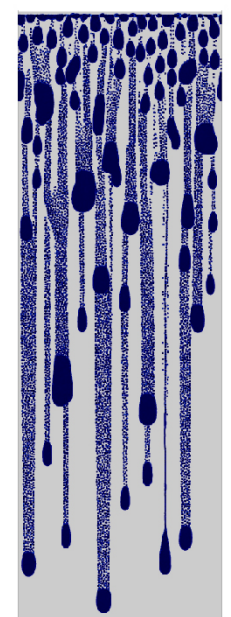

(b)

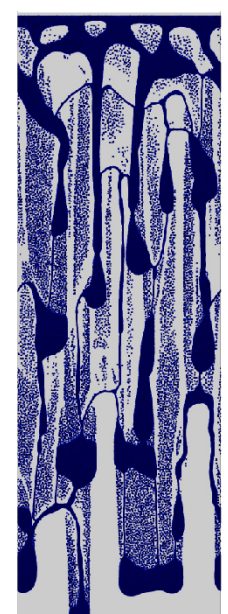

(c)

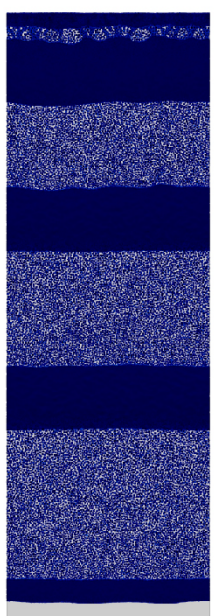

(d)

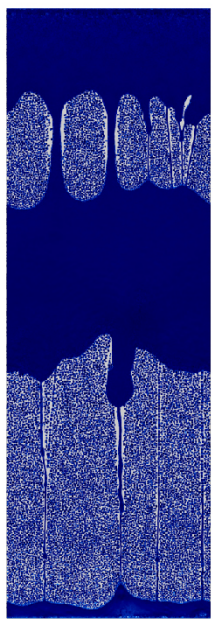

(e)

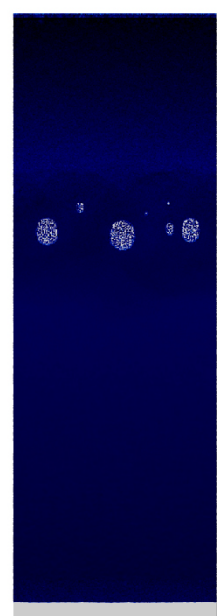

(f)

FIGURE 5.2: Flow inside smooth fractures (six flow rates, from left to right): (a) $Q=4 \times 10^{-6} \mathrm{~m}^{3} / \mathrm{s}, t=1.44 \mathrm{~s}$; (b) $Q=8 \times 10^{-6} \mathrm{~m}^{3} / \mathrm{s}, t=$ $1.07 \mathrm{~s}$; (c) $Q=2 \times 10^{-5} \mathrm{~m}^{3} / \mathrm{s}, t=0.74 \mathrm{~s}$; (d) $Q=6 \times 10^{-5} \mathrm{~m}^{3} / \mathrm{s}, t=$ $0.46 \mathrm{~s}$; (e) $Q=1 \times 10^{-4} \mathrm{~m}^{3} / \mathrm{s}, t=0.40 \mathrm{~s}$; (f) $Q=5.06 \times 10^{-4} \mathrm{~m}^{3} / \mathrm{s}$, $t=0.11 \mathrm{~s}$.

Figure 5.2 shows the fluid distributions inside a smooth fracture for different infiltration rates and the transition between flow regimes. For fluxes up to $Q>$ $1 \times 10^{-5} \mathrm{~m}^{3} / \mathrm{s}$, the flow mainly consists of droplets, partially leaving behind trailing films. At fluxes $Q>1 \times 10^{-5} \mathrm{~m}^{3} / \mathrm{s}$, the flow transitions into a rivulet-dominated regime with occasional occurrence of snapping droplets and/or rivulets. Similar flow regimes were observed in our previous SPH studies (Kordilla et al.; 2017; Kordilla J.; 2013). In this work, we extend our studies to observe a transition between rivulets and film flow. For higher flow rates, $Q>4 \times 10^{-5} \mathrm{~m}^{3} / \mathrm{s}$, we observe the formation of snapping films, which extend throughout the periodic boundaries on the lateral sides of the domain. Finally, a stable fluid front develops for $Q=$ $5.06 \times 10^{-4} \mathrm{~m}^{3} / \mathrm{s}$ (Fig. 5.2f) in accordance with the theoretical condition (Eq. (5.22)).

To quantify the instability of the fluid front, we calculate the standard deviation $s$ of velocity within each section $v_{\text {sec }}^{i}$ with respect to the mean flow velocity $\bar{v}$ (Table 5.1). Squares in Figure 5.6 show the standard deviations for each infiltration rate. The largest $s^{s}=0.0813$ is observed for $Q=8 \times 10^{-6} \mathrm{~m}^{3} / \mathrm{s}$. For $Q \geq 8 \times 10^{-6} \mathrm{~m}^{3} / \mathrm{s}$, the standard deviation decreases and reaches its minimum value $s^{s}=0.002$ when a 
stable fluid front is developed at $Q=5.06 \times 10^{-4} \mathrm{~m}^{3} / \mathrm{s}$. For $Q>5.06 \times 10^{-4} \mathrm{~m}^{3} / \mathrm{s}$, the standard deviation remains equal to its minimum value.

\subsubsection{Flow in rough vertical fractures}

Fractures encountered in geological environments have rough surfaces, despite the fact that they are often approximated as smooth in, for example, discrete fracture network modeling (Hyman et al.; 2015; Therrien and Sudicky; 1996b). In our previous work employing the PF-SPH model (Kordilla et al.; 2017; Kordilla J.; 2013; Shigorina et al.; 2017), we investigated various effects of surface roughness on droplet flow and wetting dynamics, including capillary-Bond number scaling functions, onset of trailing film formation, relation of microscale and macroscopic hydropho$\mathrm{bic} /$ hydrophilic behavior for Cassie and Wenzel droplets, and the effect of roughness anisotropy on droplet flows. In this work, we extend these studies to investigate the effect of roughness on the behavior of highly complex flows with flow regimes ranging from droplet to droplet-to-rivulet and rivulet-to-film flows.

Following Tartakovsky and Meakin (2005b) and Kordilla J. (2013), we create rough fractures characterized by the Hurst exponent $\zeta$ (Bouchaud et al.; 1990) and an initial maximum value $\Delta$ for the random displacement from a planar surface. It was shown that $\zeta$ often assumes values of $0.80(5)$ for consolidated impermeable rocks (Bouchaud; 1997; Ponson et al.; 2006), however, wider ranges of $0<\zeta<0.9$ have been measured as well (Boffa et al.; 1998; Sahimi; 2011).

For our simulations, we create two rough fractures with dimensions $10 \times 30 \mathrm{~cm}$, $\zeta=0.75$, and $\Delta=10.0$ and $20.0 \mathrm{~mm}$. The fracture aperture is $b=2.0 \mathrm{~mm}$. Figure 5.1 (middle and right) shows the employed fractures, where light colors represent elevated parts of the height field and dark colors are associated with depressions.

Figures 5.3 and 5.4 show the fluid distributions inside the rough fractures with $\Delta=10.0$ and $20.0 \mathrm{~mm}$ for different infiltration rates. Compared to the flow in a smooth fracture from section 5.3.1, a stable fluid front is established for a flux of $Q=5.06 \times 10^{-4} \mathrm{~m}^{3} / \mathrm{s}$ (Figs. $5.3 \mathrm{f}$ and $5.4 \mathrm{f}$ ).

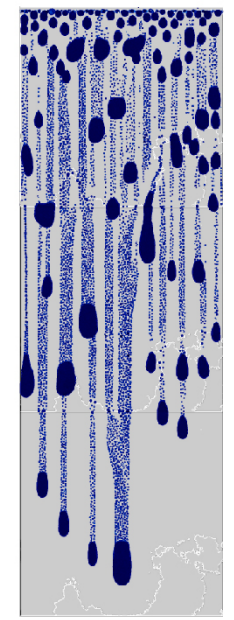

(a)

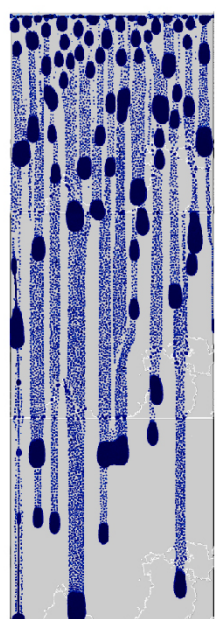

(b)

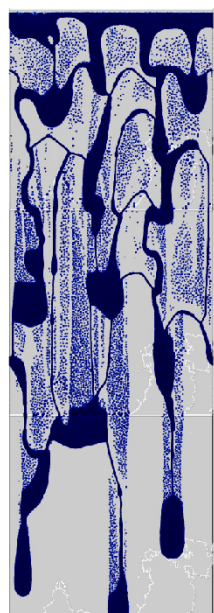

(c)

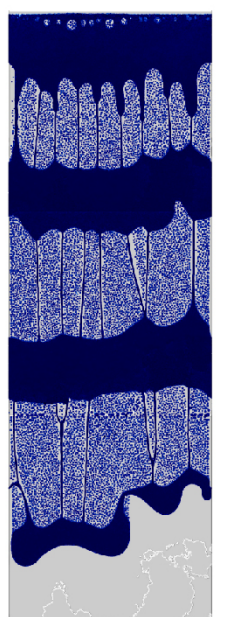

(d)

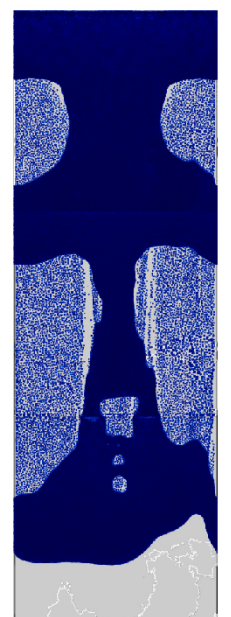

(e)

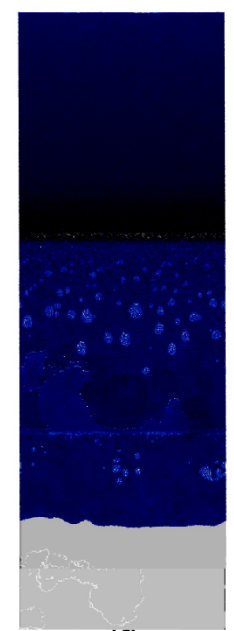

(f)

FIGURE 5.3: Flow inside rough fractures with $\Delta=10.0 \mathrm{~mm}$ (six flow rates, from left to right): (a) $Q=4 \times 10^{-6} \mathrm{~m}^{3} / \mathrm{s}, t=1.65 \mathrm{~s}$; (b) $Q=$ $8 \times 10^{-6} \mathrm{~m}^{3} / \mathrm{s}, t=1.19 \mathrm{~s}$; (c) $Q=2 \times 10^{-5} \mathrm{~m}^{3} / \mathrm{s}, t=0.74 \mathrm{~s}$; (d) $Q=$ $6 \times 10^{-5} \mathrm{~m}^{3} / \mathrm{s}, t=0.46 \mathrm{~s}$; (e) $Q=1 \times 10^{-4} \mathrm{~m}^{3} / \mathrm{s}, t=0.40 \mathrm{~s}$; (f) $Q=$ $5.06 \times 10^{-4} \mathrm{~m}^{3} / \mathrm{s}, t=0.12 \mathrm{~s}$. 


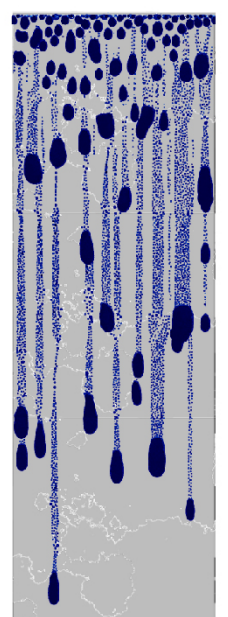

(a)

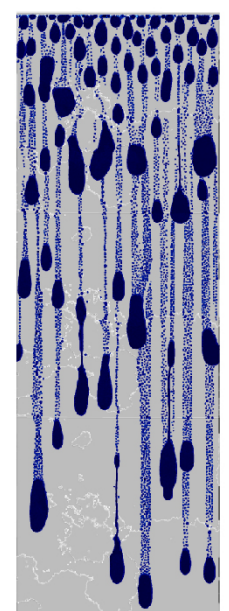

(b)

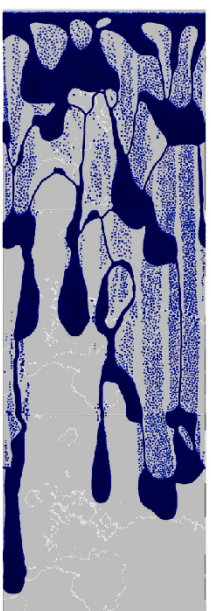

(c)

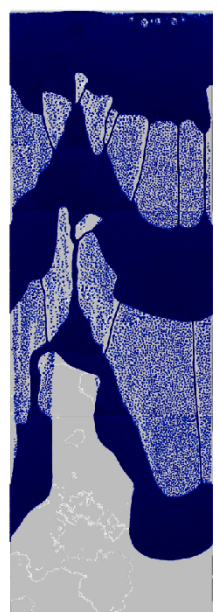

(d)

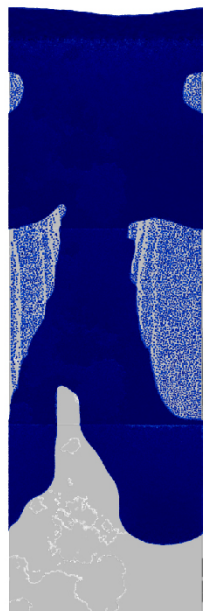

(e)

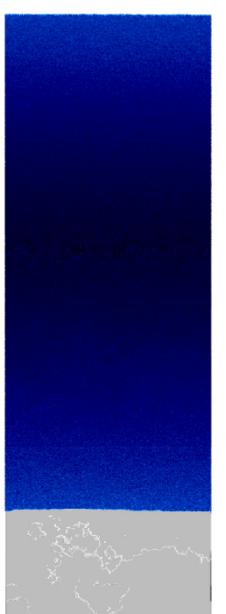

(f)

FIGURE 5.4: Flow inside rough fractures with $\Delta=20.0 \mathrm{~mm}$ (six flow rates, from left to right): (a) $Q=4 \times 10^{-6} \mathrm{~m}^{3} / \mathrm{s}, t=1.65 \mathrm{~s}$; (b) $Q=$ $8 \times 10^{-6} \mathrm{~m}^{3} / \mathrm{s}, t=1.19 \mathrm{~s}$; (c) $Q=2 \times 10^{-5} \mathrm{~m}^{3} / \mathrm{s}, t=0.74 \mathrm{~s}$; (d) $Q=$ $6 \times 10^{-5} \mathrm{~m}^{3} / \mathrm{s}, t=0.46 \mathrm{~s}$; (e) $Q=1 \times 10^{-4} \mathrm{~m}^{3} / \mathrm{s}, t=0.40 \mathrm{~s}$; (f) $Q=$ $5.06 \times 10^{-4} \mathrm{~m}^{3} / \mathrm{s}, t=0.12 \mathrm{~s}$.

TABLE 5.1: Statistical properties for various fluxes in smooth and rough fractures.

\begin{tabular}{lccccccc}
\hline & \multicolumn{2}{c}{ smooth } & & \multicolumn{4}{c}{ rough } \\
\cline { 2 - 3 } $\mathrm{Q}\left(\mathrm{m}^{3} / \mathrm{s}\right)$ & $\bar{v}^{s}$ & $s^{s}$ & & $\bar{v}_{\Delta=10}^{r}$ & $s_{\Delta=10}^{r}$ & $\bar{v}_{\Delta=20}^{r}$ & $s_{\Delta=20}^{r}$ \\
\hline $4 \times 10^{-6}$ & 0.15 & 0.0346 & & 0.13 & 0.0368 & 0.12 & 0.0387 \\
$8 \times 10^{-6}$ & 0.25 & 0.0813 & & 0.21 & 0.0927 & 0.23 & 0.0956 \\
$2 \times 10^{-5}$ & 0.41 & 0.0291 & & 0.36 & 0.0433 & 0.32 & 0.0477 \\
$4 \times 10^{-5}$ & 0.55 & 0.0154 & & 0.50 & 0.0251 & 0.43 & 0.0339 \\
$6 \times 10^{-5}$ & 0.69 & 0.0035 & & 0.61 & 0.0047 & 0.53 & 0.0075 \\
$1 \times 10^{-4}$ & 0.89 & 0.0023 & & 0.71 & 0.0036 & 0.65 & 0.0061 \\
$3 \times 10^{-4}$ & 1.63 & 0.0021 & & 1.55 & 0.0033 & 1.45 & 0.0039 \\
$5 \times 10^{-4}$ & 2.53 & 0.0020 & & 2.50 & 0.0030 & 2.41 & 0.0032 \\
$8 \times 10^{-4}$ & 3.69 & 0.0020 & & 3.60 & 0.0030 & 3.50 & 0.0031 \\
\hline
\end{tabular}

Figures 5.5 and 5.6 report the average flow velocities and standard deviations in the rough fractures as a function of $\Delta$ and $Q$, and Table 5.1 summarizes the velocities and standard deviation values in the smooth and rough fractures for all studies of $Q$. It is evident that velocities in rough fractures are smaller than the corresponding velocities in the smooth fracture. The standard deviation of velocity is larger for rough fractures than for smooth fractures due to enhanced flow focusing (fingering) in roughness-induced channel structures.

\subsubsection{Comparison with analog experiments}

In this section we consider the experimental results reported by Nicholl and Glass (2005), who studied the fluid infiltration in initially dry fractures. For the experiment a textured glass fracture with dimensions $30 \times 60 \mathrm{~cm}$ was used. The aperture of the fracture is $b=0.2255 \mathrm{~mm}$. In the experiment an averaged velocity $\bar{v}_{\mathrm{f}}$ of individual fingers was investigated as a function of a supply rate $Q$ (Nicholl and Glass; 2005, 


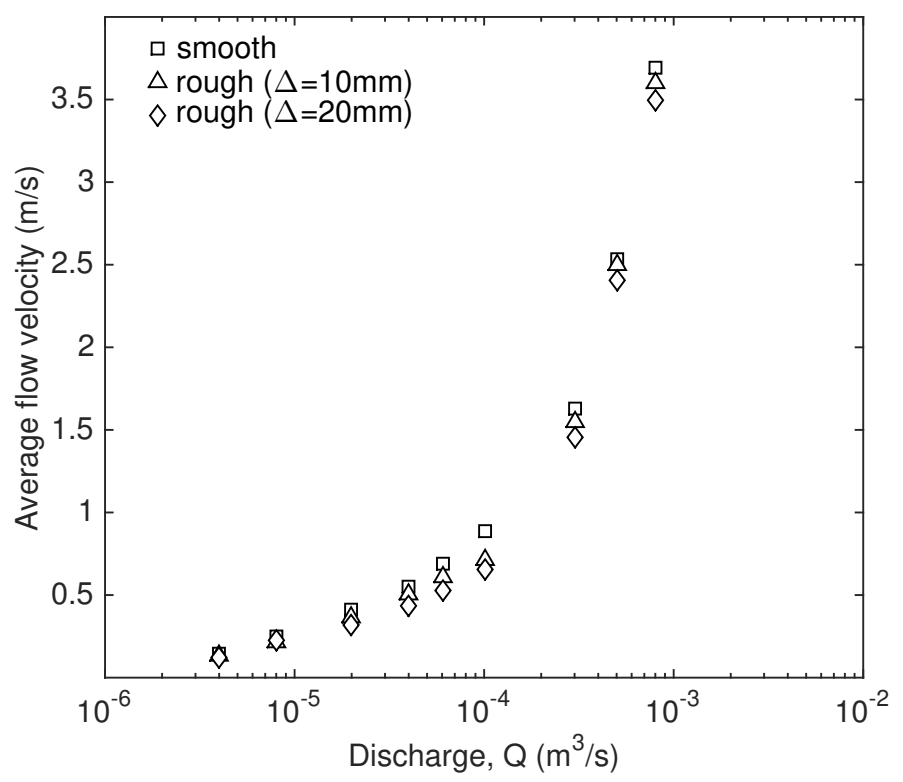

FIGURE 5.5: Average flow velocities in smooth and rough fractures for different infiltration rates.

Fig. 27). To compare this experiment with our simulations, we calculate the specific discharge $q$ and the normalized velocity $v_{\Delta=10,20}^{s, r, *}, v_{\mathrm{f}}^{*}$ :

$$
\begin{gathered}
q=\frac{Q}{b d}, \\
v_{\Delta=10,20}^{s, r, *}, v_{\mathrm{f}}^{*}=\frac{\bar{v}, \bar{v}_{\mathrm{f}}}{b},
\end{gathered}
$$

where $d$ is the fracture width, $v_{\Delta=10,20}^{s, r, *}$ is the normalized averaged flow velocity from simulations, the superscripts $s$ and $r$ represent smooth and rough fractures respectively, $v_{\mathrm{f}}^{*}$ is the normalized velocity of individual fingers from experiments. The experimental and simulation data are listed in Table 5.2.

TABLE 5.2: Comparison of experimental (Nicholl and Glass; 2005,

\begin{tabular}{|c|c|c|c|c|c|}
\hline \multicolumn{2}{|c|}{ Experiment } & \multicolumn{4}{|c|}{ Simulation } \\
\hline$q$ & $v_{\mathrm{f}}^{*}$ & $q$ & $v^{s, *}$ & $v_{\Delta=10}^{r, *}$ & $v_{\Delta=20}^{r, *}$ \\
\hline $1.7 \times 10^{-5}$ & 7.39 & 0.02 & $7.6 \times 10^{1}$ & $6.4 \times 10^{1}$ & $6.0 \times 10^{1}$ \\
\hline $3.7 \times 10^{-5}$ & 9.24 & 0.04 & $1.2 \times 10^{2}$ & $1.2 \times 10^{2}$ & $1.1 \times 10^{2}$ \\
\hline $7.4 \times 10^{-5}$ & 13.3 & 0.10 & $2.4 \times 10^{2}$ & $1.8 \times 10^{2}$ & $1.6 \times 10^{2}$ \\
\hline $1.7 \times 10^{-4}$ & 20.7 & 0.20 & $2.7 \times 10^{2}$ & $2.5 \times 10^{2}$ & $2.1 \times 10^{2}$ \\
\hline $3.7 \times 10^{-4}$ & 29.6 & 0.30 & $3.4 \times 10^{2}$ & $3.0 \times 10^{2}$ & $2.8 \times 10^{2}$ \\
\hline $7.4 \times 10^{-4}$ & 37.0 & 0.50 & $4.5 \times 10^{2}$ & $3.6 \times 10^{2}$ & $3.3 \times 10^{2}$ \\
\hline $9.9 \times 10^{-4}$ & 48.0 & 1.50 & $8.2 \times 10^{2}$ & $7.8 \times 10^{2}$ & $7.3 \times 10^{2}$ \\
\hline $1.48 \times 10^{-3}$ & 59.1 & 2.53 & $1.3 \times 10^{3}$ & $1.2 \times 10^{3}$ & $1.2 \times 10^{3}$ \\
\hline $2.46 \times 10^{-3}$ & 81.3 & 4.00 & $1.9 \times 10^{3}$ & $1.8 \times 10^{3}$ & $1.8 \times 10^{3}$ \\
\hline
\end{tabular}

Fig. 27) and simulation data.

Figure 5.7 shows $v_{\mathrm{f}}^{*}$ as a function of $q$. On the logarithmic plot, $\log v_{\mathrm{f}}^{*}$ shows 


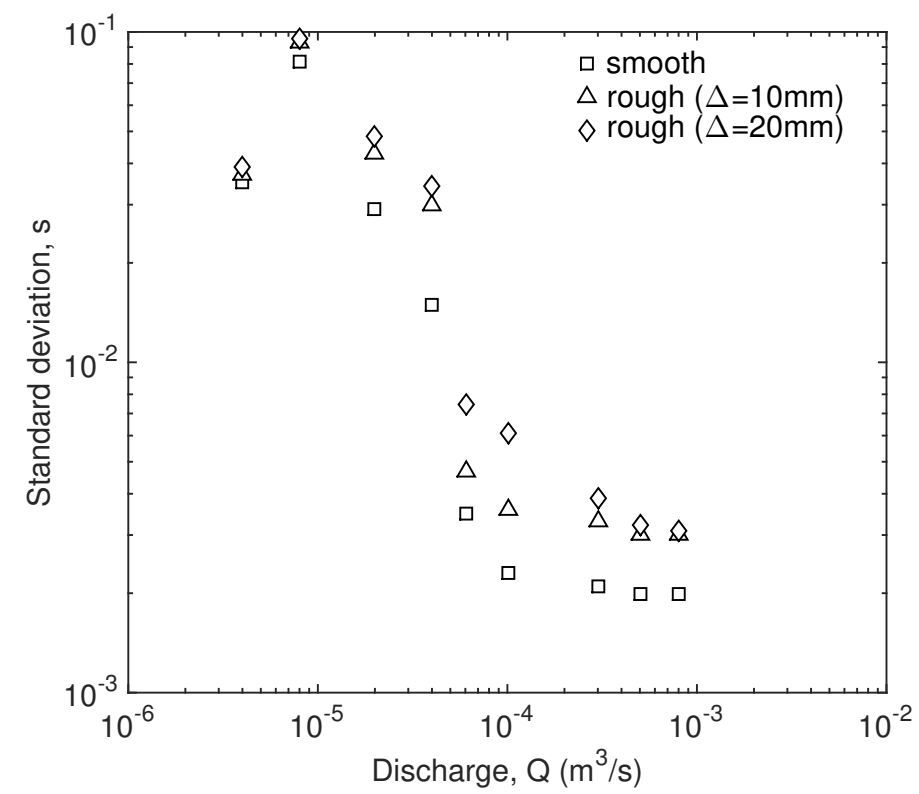

FIGURE 5.6: Standard deviations of flow velocities in smooth and rough fractures for different infiltration rates.

linear dependence on $\log q$ that assumes the power law dependence $v^{*}=q^{p}$. A similar power law dependence was observed in the experiments with $p=0.48$. In our simulations, the exponent $p$ lies in the range between 0.50 and 0.55 for smooth and rough fractures, which is close to the experimental value of $p$ (Fig. 5.7).

Next, we investigate finger tip length $L_{\text {tip }}$ as a function of finger tip velocity and compare our simulation results with an analytical solution and experimental data from (Nicholl and Glass; 2005, Fig. 30). Here the term finger tip is often also referred to as elongated droplets or slugs. For the sake of completeness the following is an excerpt from Nicholl and Glass (2005). For a stagnant finger tip the viscous forces vanish and capillary forces balance the gravitational component, i.e.,

$$
\Delta F_{g}-\Delta F_{c}=0,
$$

where gravitational forces are approximated as

$$
\Delta F_{g} \approx \Delta \rho g \cos (\varphi) h_{g}
$$

and act over a characteristic length scale $h_{g}$. The pressure differential within the water phase of a fingertip is approximated as

$$
\Delta F_{c} \approx \sigma\left(\left(\frac{1}{r_{1}}+\frac{1}{r_{2}}\right)_{\text {front }}-\left(\frac{1}{r_{1}}+\frac{1}{r_{2}}\right)_{\text {trail }}\right),
$$

where the radius of curvature $r_{1}$ (first principal radius spanning both walls of the fracture) and the in-plane curvature $r_{2}$ at each point could not be determined in the laboratory experiments. In order to estimate the fingertip length $L_{t i p}$ from the equality Eq. (5.28) together with Eq. (5.29) and (5.30) Nicholl and Glass (2005) assume that the pressure along the leading and trailing edge can be approximated by the wetting and draining pressure head $\left(\psi_{w}\right.$ and $\left.\psi_{d}\right)$ such that

$$
L_{t i p}=\left(\psi_{w}-\psi_{d}\right) / \cos (\varphi) .
$$




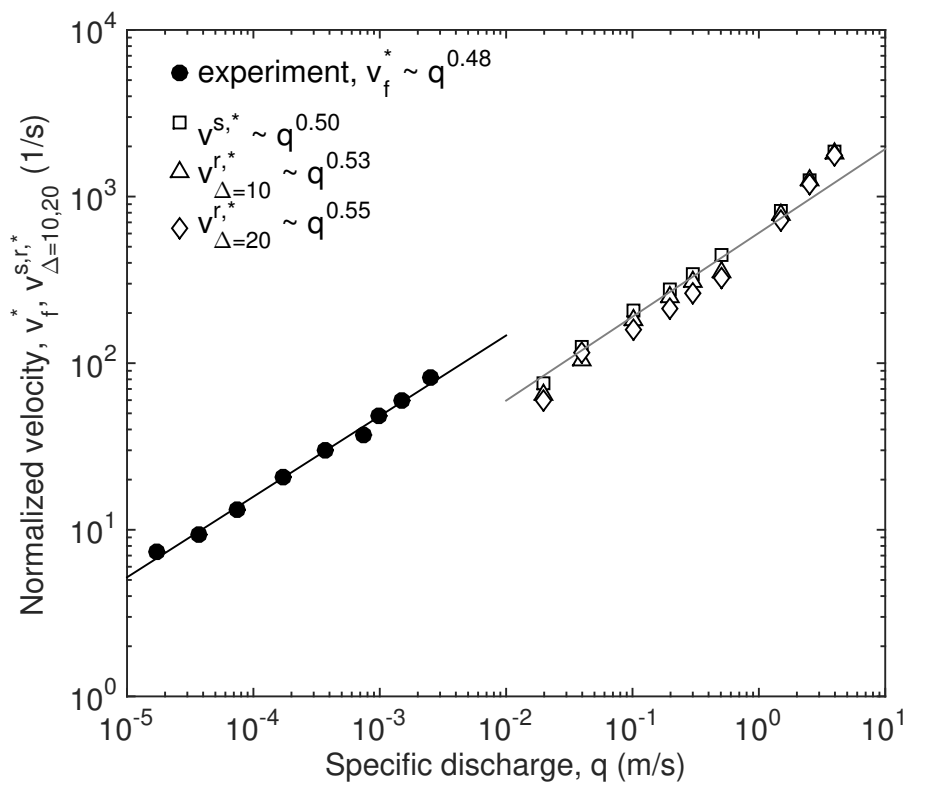

FIGURE 5.7: Comparison of the normalized averaged flow velocity from PF-SPH simulations with the normalized averaged velocity $\bar{v}_{\mathrm{f}}$ of individual fingers from experiments (Nicholl and Glass; 2005, Fig.

27). (black line) $p=0.5$, (grey line) $p=0.53$.

Further they found that for a vertical fracture $(\cos (\varphi)=1)$ the measured stagnant fingertip lengths are very close to the values obtained from Eq. (5.31). Hence, for our simulations we define

$$
\psi_{w}-\psi_{d}=\bar{L}_{t i p},
$$

where $\bar{L}_{t i p}$ is the average length of stagnant finger tips found to be $1.0 \mathrm{~cm}$ in our simulations.

Under dynamic conditions viscous forces are introduced into the force balance which now becomes

$$
\Delta F_{g}-\Delta F_{c}-\Delta F_{v}=0 .
$$

Viscous forces act over a characteristic length scale $h_{v}$ and are approximated by

$$
\Delta F_{v} \approx v \mu h_{v}\left(k k_{r}\right)^{-1}
$$

where $k_{r}=1$ under the assumption that fingertips are fully saturated and $v$ is the fingertip velocity. Insertion of Eq. (5.29), (5.30) and (5.34) into Eq. (5.33) and assuming the characteristic length scales correspond to the fingertip length, i.e., $L_{t i p}=h_{g}=h_{v}$, the following expression for the fingertip length is obtained:

$$
L_{\text {tip }}=\frac{\psi_{w}-\psi_{d}}{\cos (\varphi)}\left(1-\frac{v}{K_{s} k_{r} \cos (\varphi)}\right)^{-1}=\bar{L}_{\text {tip }}\left(1-\frac{v}{K_{s}}\right)^{-1} .
$$

As the wetting pressure is dependent on the fingertip velocity the above equation can only serve as a first-order approximation. Nicholl and Glass (2005) found a better fit to their data using the following expression

$$
L_{t i p}=\frac{\psi_{w}\left(1-\hat{v}^{\epsilon}\right)-\psi_{d}}{(1-\hat{v}) \cos (\varphi)}
$$


which takes into account the dependence of the leading contact angle (related to the dynamic wetting pressure $\psi_{w}$ ) on velocity, where $\epsilon=0.1$ is an empirical coefficient.

Figure 5.8 shows the simulation data and experimental results of Nicholl and Glass (2005) (Fig. 30 therein), as well as the analytical solutions Eq. (5.35) and (5.36) for the scaled fingertip length $\left(L_{\text {tip }} \cos \varphi\right)$ as a function of scaled fingertip velocity $\hat{v}=v / K_{s} k_{r} \cos \varphi$. The analytical solution Eq. (5.35) shows a good fit with simulation data for $\bar{L}_{t i p}=\left(\psi_{w}-\psi_{d}\right)=1.0 \mathrm{~cm}$ and $\epsilon=0.1$. Similar to Nicholl and Glass (2005), we also observe higher discrepancies between the analytical solution and the simulations results for intermediate values of the scaled velocity $\hat{v}$.

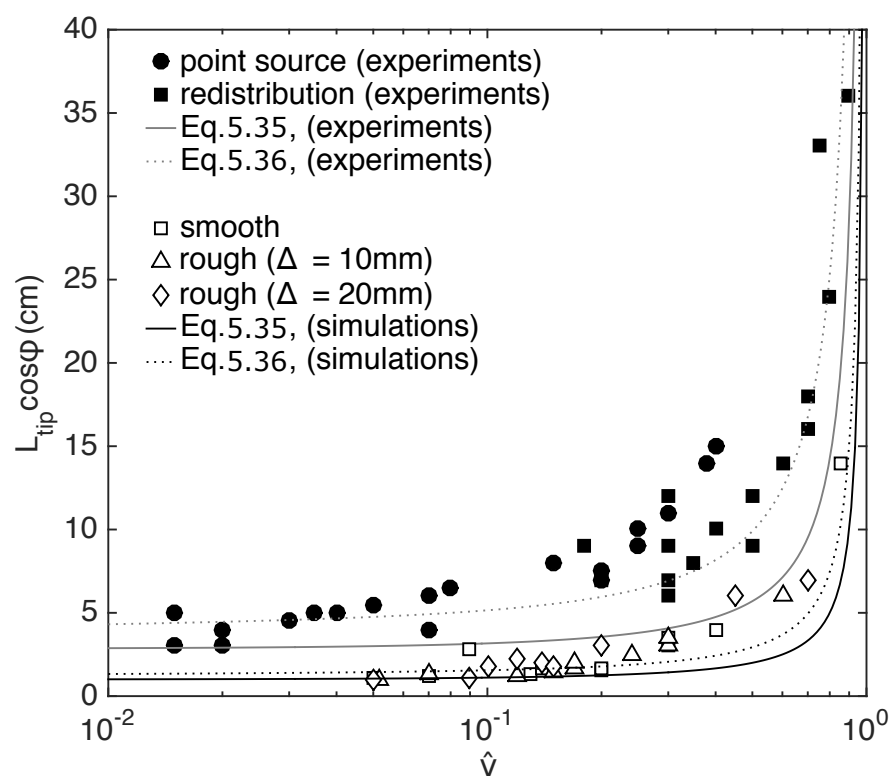

FIGURE 5.8: Comparison of the scaled fingertip length $\left(L_{\text {tip }} \cos \varphi\right)$ as a function of scaled finger velocity $\hat{v}=v / K_{s} k_{r} \cos \varphi$ for simulations and experiment from Nicholl and Glass (2005). For the analytical solutions $\bar{L}_{t i p}=2.85 \mathrm{~cm}$ and $\epsilon=0.1$ (experiments) and $\bar{L}_{t i p}=1.0 \mathrm{~cm}$ and $\epsilon=0.1$ (simulations).

\subsection{Discussion}

In our previous work (Shigorina et al.; 2017), we observed that, depending on its geometry and orientation, a "structured" surface roughness can accelerate or decelerate flow velocity. In this work, we create random rough fracture surfaces with $\zeta=0.75$ and $\Delta=10.0$ and $20.0 \mathrm{~mm}$, to approximate natural rough fracture surfaces. Our simulation results show that with increasing $\Delta$, the velocity $\bar{v}$ decreases for all infiltration rates (Fig. 5.5, Table 5.1). For a given infiltration rate, the average velocity $\bar{v}$ in rough fractures can be 1.4 times lower than $\bar{v}$ in the smooth fracture.

Here, we demonstrate the influence of fracture roughness and viscous and capillary forces on flow instability. Our simulations show that even in a smooth fracture, fluid flow is unstable for $Q<5 \times 10^{-5} \mathrm{~m}^{3} / \mathrm{s}$ (Fig. 5.2a-c). For $Q$ between $5 \times 10^{-5}$ and $5 \times 10^{-4} \mathrm{~m}^{3} / \mathrm{s}$, the evolving fluid front appears stable (straight) (Fig. $5.2 \mathrm{~d}, \mathrm{e}$ ). However, because of fluid snapping and merging, the fluid flow velocities may significantly fluctuate. For the rough fractures, the fluid front is unstable for $Q<$ $5 \times 10^{-4} \mathrm{~m}^{3} / \mathrm{s}$ (Figs. 5.3a-e and 5.4a-e). For $Q>5 \times 10^{-4} \mathrm{~m}^{3} / \mathrm{s}$, the fluid front is 
stable for both rough and smooth fractures, and the velocity $\bar{v}$ is constant (Figs. 5.2f, 5.3f, and 5.4f).

To characterize the instability, we calculate standard deviations of flow velocity $s$ (Fig. 5.6, Table 5.1). Unstable flows are characterized by rivulets (or fingering flow) and/or droplet streams in parts of the fracture, while the remaining fracture is dry with flow velocities close to zero. We observe a peak of standard deviation for a discharge $Q=8 \times 10^{-6} \mathrm{~m}^{3} / \mathrm{s}$ (Fig. 5.6). At a discharge $Q=8 \times 10^{-6} \mathrm{~m}^{3} / \mathrm{s}$ approximately $50 \%$ of the fracture is saturated (exhibiting high flow velocities), and $50 \%$ of the fracture is dry with very low velocity or no flow occurring. Hence, the distribution of flow velocities gives rise to the highest standard velocity deviation relative to the mean flow velocity. According to Eq. (5.22) and in accordance with our results for fluxes approaching $Q=5.04 \times 10^{-4} \mathrm{~m}^{3} / \mathrm{s}$ the standard deviation of velocity decreases, as the number of instabilities (i.e. rivulets or droplet pathways) decreases and hence the likelihood of localized preferential flow paths declines. For flows $Q<8 \times 10^{-6} \mathrm{~m}^{3} / \mathrm{s}$ the standard deviation is decreasing again as the number of individual streams is approaching a maximum (this has for example been studied by Ghezzehei (2005) using an energy-minimization principle for free-surface systems) and therefore the (bulk) system exhibits a more diffuse behavior again. It should be noted that for even lower injection rates (that we haven't studied in this work) the flow may even be bounded by only one side of the fracture such that slugs (i.e. droplets bounded by both fracture walls) may be transformed into "true" droplets and for even lower flow rates thin films bounded by only one side of the fracture wall may occur. This transition has been studied for example by Ghezzehei (2005) and is most likely characterized by a different behavior of the velocity standard deviation. Our results show that with increasing $\Delta$, the standard deviation $s$ increases for all infiltration rates due to the increasing likelihood of finger and droplet stream formation. Fluid front instabilities create preferential flow pathways, which impacts the fracture-matrix interaction area, wetting dynamics, infiltration processes, and arrival times of tracers or contaminants in the vadose zone.

However, in fully saturated smooth and rough fractures, the standard deviation $s$ reaches its minimum value. As expected, in the fully saturated fractures, the mean flow velocity $\bar{v}$ is equal to the infiltration rate.

The comparison of our simulation to the experiments of Nicholl and Glass (2005) show good agreement for two of the main characteristics of the experimental setup. Despite the differences in geometry (our fractures are smaller but with larger aperture) the scaling of normalized velocities with specific discharge show similar trends and the scaling of fingertip length with scaled velocity agree with the analytical predictions.

In our simulations, the scaling of normalized velocities $v_{\Delta=10,20}^{s, r, *}$ and specific discharge, $v^{*} \sim q^{p}$, yields exponents on the order of 0.5 to 0.55 (the exponent is increasing with increasing roughness) compared to $p=0.48$ in the laboratory experiments. The simulations cover a slightly higher range of normalized velocities, however, extrapolated normalized velocities for the given scaling exponents (Fig. 5.7) and lower velocity ranges are expected to be below the experimental ones. The main reason for this is that flow velocities from the simulations are averaged along the whole fracture, while in the experiment only velocities of individual rapid fingers were measured.

The comparison of fingertip length and scaled fingertip velocities between experiments and simulations proves that the model is able to represent the complex case of a gravity-driven instability. The values of $L_{\text {tip }}$ for the experiment are larger than the simulated ones. This is mainly caused by the wider aperture employed in the 
simulations, which triggers faster snapping of rivulets due to the increase of gravitational force compared to the capillary component, hence, the resulting fingers are smaller.

\subsection{Conclusion}

In this work, we employed a three-dimensional PF-SPH model to simulate gravitydriven flow in smooth and rough fractures. Two types of rough fractures with $\zeta=$ 0.75 , and $\Delta=10.0$ and $20.0 \mathrm{~mm}$ were considered.

To study the effect of fracture roughness on the front instability and flow regimes, the fluid was injected in fractures with a constant flux, and the average flow velocity and standard deviation were computed for different infiltration rates.

Our results indicated that the flow velocity in smooth and rough fractures increases with an increasing infiltration rate. We observe the transition between flow regimes, which has been validated in our earlier studies via laboratory and numerical experiments (Kordilla et al.; 2017). For both smooth and rough fractures, the rivulet flow regime is dominant for $Q \leq 4 \times 10^{-5} \mathrm{~m}^{3} / \mathrm{s}$, while, for higher fluxes, a snapping or continuous film was observed. For the smooth fracture, the fluid front remained stable for $Q>4 \times 10^{-5} \mathrm{~m}^{3} / \mathrm{s}$, while, for rough fractures, the fluid film front formed some preferential pathways. For the fluxes $Q>5 \times 10^{-4} \mathrm{~m}^{3} / \mathrm{s}$, the whole fracture filled with water, and the fluid profile was stable for rough and smooth fractures. Furthermore, we observed deceleration of flow velocity due to roughness. Average velocities $\bar{v}^{r}$ in rough fractures were smaller than the corresponding velocities $\bar{v}^{s}$ in smooth fractures.

Our results show that the largest velocity variance $s$ (a measure of front instability) occurs for $Q=8 \times 10^{-6} \mathrm{~m}^{3} / \mathrm{s}$ in both the smooth and the rough fractures. For $Q>8 \times 10^{-6} \mathrm{~m}^{3} / \mathrm{s}$, the standard deviation decreases and reaches its minimum value when a stable fluid front developed. Because of the roughness inducing flow channeling effects, for a given $Q$, the standard deviation $s^{r}$ in rough fractures was larger than $s^{s}$ in smooth fractures.

The analysis of flux-dependent fluid flow velocity and instability in smooth and rough vertical fractures is required to gain a better understanding of the formation of preferential flow paths. Because of their flow-focusing properties, such fractures not only contribute to the fracture-specific flow path, but are important to understand large-scale hydro(geo)logical problems, i.e., infiltration/percolation through whole fracture networks and associated processes, such as aquifer recharge or contaminant transport (vulnerability). Large-scale fracture networks and connections between fractures induce a variety of additional partitioning and dispersion processes. Preferential flow paths along fracture networks exhibit depth-dependent reshaping of the flow and input signal due to flow rate specific partitioning dynamics at fracture intersections (e.g., Kordilla et al.; 2017), and hence, understanding the distribution of flow within each fracture is an important factor in defining the partitioning dynamics for fracture cascades/networks (Noffz et al.; 2018).

For saturated systems promising extension of the classical cubic law are for example applied to create two-dimensional depth-averaged representations of complex three-dimensional flows via dimension reduction in individual fractures (Modified Local Cubic Law, Wang et al.; 2015). Unsaturated flows in fractures exhibit an even higher degree of internal complexity and in order to bridge the gap between large-scale applications (for example DFN models) and small-scale process oriented 
models of unsaturated flows in fractures further developments and studies are required to enhance existing solutions (Dippenaar and Van Rooy; 2016).

Convergence of large-scale applications and process oriented models may also be facilitated by an increase in numerical efficiency and/or computing power. Nonuniform resolution (or adaptivity) in SPH is not yet very well-established and has been defined as one of the most important goals within the SPH community. Adaptive resolutions in principle only require particle-dependent sizes of $h$ (and modifications of the kernel computation when particles of different size interact) in order to account for variable particle number densities (volumes). This is rather straightforward for static grids (e.g. mesh-based approaches) and solid SPH boundaries but becomes challenging once highly dynamic fluid interfaces in the Lagrangian framework are considered. In order to apply non-uniform resolution to such flows, adaptive splitting and coalescence schemes are required and consequently a set of splitting/coalescence conditions, which are challenging to define for free-surface flows in the presence of surface tension.

Despite methodological advances (e.g. dynamic non-uniform resolution) and increase in computing power the simulation of unsaturated flows through whole fracture networks (field scales with millions or billions of fractures in 3D) is not possible with any numerical method that discretizes the Navier-Stokes equation in the near future. On fracture scales, computational costs are largely affected by the highly anisotropic properties with respect to the resolved length scales. In-plane dimensions of fractures are generally orders of magnitude larger (centimeters to meters) than aperture widths (micrometers to millimeters) which consequently control the resolution required to discretize the flow field along the fracture normals as well as the height-dependent roughness field. Vertically depth-integrated approaches such as the modified local cubic law model (Wang et al.; 2015) are computationally more efficient, however, they fail to resolve saturated and unsaturated flow processes in the presence of preferential flow paths which may induce strong hydraulic contrasts, especially for wide aperture fractures. Here free-surface flows, that is flows bounded by only one fracture surface, may occur and hence invalidate or limit the application of vertically depth-integrated modeling approaches.

For now the outcomes of such discrete simulations presented in this paper can only serve as a tool to identify the importance of individual fracture-scale processes and inter-fracture partitioning dynamics in order to enhance DFN models or even upscaled lumped parameter models (Liu et al.; 1998, 2003).

\section{Acknowledgments.}

This work was funded by the Deutsche Forschungsgemeinschaft (DFG; German Research Foundation) under grant KO 5359/1-1 and SA 501/26-1. A.M. Tartakovsky was supported by the Department of Energy (DOE)'s Office of Advanced Scientific Computing Research and Pacific Northwest National Laboratory (PNNL). PNNL is operated by Battelle for the DOE under Contract DE-AC05-76RL0183

\section{Bibliography}

Batchelor, G. K. (1967). An introduction to fluid dynamics, Cambridge University Press, Cambridge, UK.

Boffa, J. M., Allain, C. and J.P Hulin (1998). Experimental analysis of fracture rugosity in granular and compact rocks, The European Physical Journal Applied Physics 2(3): 281-289.

URL: $h t t p: / / w w w . e p j a p . o r g / 10.1051 / e p j a p: 1998194$ 
Bouchaud, E. (1997). Scaling properties of cracks, Journal of Physics: Condensed Matter 9(21): 4319.

Bouchaud, E., Lapasset, G. and Planes, J. (1990). Fractal dimension of fractured surfaces: a universal value?, EPL (Europhysics Letters) 13(1): 73.

Briggs, S., Karney, B. W. and Sleep, B. E. (2017). Numerical modeling of the effects of roughness on flow and eddy formation in fractures, Journal of Rock Mechanics and Geotechnical Engineering 9(1): 105-115.

Brown, S., Caprihan, A. and Hardy, R. (1998). Experimental observation of fluid flow channels in a single fracture, Journal of Geophysical Research: Solid Earth 103(B3): 5125-5132.

Cacas, M. C., Ledoux, E., de Marsily, G., Tillie, B., Barbreau, A., Durand, E., Feuga, B. and Peaudecerf, P. (1990). Modeling fracture flow with a stochastic discrete fracture network: calibration and validation: 1 . The flow model, Water Resources Research 26(3): 479-489.

URL: $h$ ttp://doi.wiley.com/10.1029/WR026i003p00479

Cueto-Felgueroso, L. and Juanes, R. (2008). Nonlocal interface dynamics and pattern formation in gravity-driven unsaturated flow through porous media, Physical Review Letters 101(24): 244504.

Dershowitz, W. S. and Einstein, H. H. (1988). Characterizing rock joint geometry with joint system models, Rock Mechanics and Rock Engineering 21(1): 21-51.

DiCarlo, D. A., Bauters, T. W., Darnault, C. J., Steenhuis, T. S., Parlange, J. et al. (1999). Lateral expansion of preferential flow paths in sands, Water Resources Research 35(2): 427-434.

Dippenaar, M. A. and Van Rooy, J. L. (2016). On the cubic law and variably saturated flow through discrete open rough-walled discontinuities, International Journal of Rock Mechanics and Mining Sciences 89: 200-211.

Eker, E. and Akin, S. (2006). Lattice boltzmann simulation of fluid flow in synthetic fractures, Transport in Porous Media 65(3): 363-384.

Evans, D. D. and Rasmussen, T. C. (1991). Unsaturated flow and transport through fractured rock related to high-level waste repositories, Technical report, Nuclear Regulatory Commission.

Ganzenmüller, G. C., Steinhauser, M. O., Van Liedekerke, P. and Leuven, K. U. (2011). The implementation of smooth particle hydrodynamics in LAMMPS.

Geyer, T., Birk, S., Liedl, R. and Sauter, M. (2008). Quantification of temporal distribution of recharge in karst systems from spring hydrographs, Journal of Hydrology 348(3-4): 452-463.

Ghezzehei, T. (2004). Constraints for flow regimes on smooth fracture surfaces, Water Resources Research 40(11).

Ghezzehei, T. A. (2005). Flow diversion around cavities in fractured media, Water resources research $\mathbf{4 1}(11)$. 
Heilweil, V. M., Benoit, J. and Healy, R. W. (2015). Variably saturated groundwater modelling for optimizing managed aquifer recharge using trench infiltration, Hydrological Processes 29(13): 3010-3019.

Hendrickx, J. M. and Flury, M. (2001). Uniform and preferential flow mechanisms in the vadose zone, Conceptual models of flow and transport in the fractured vadose zone pp. $149-187$.

Holmes, D. W., Williams, J. R. and Tilke, P. (2011). Smooth particle hydrodynamics simulations of low reynolds number flows through porous media, International Journal for Numerical and Analytical Methods in Geomechanics 35(4): 419-437.

Hyman, J. D., Karra, S., Makedonska, N., Gable, C. W., Painter, S. L. and Viswanathan, H. S. (2015). DfnWorks: A discrete fracture network framework for modeling subsurface flow and transport, Computers and Geosciences 84: 10-19. URL: $h t t p: / / d x$.doi.org/10.1016/j.cageo.2015.08.001

Jones, B. R., Brouwers, L. B. and Dippenaar, M. A. (2018). Partially to fully saturated flow through smooth, clean, open fractures: qualitative experimental studies, $\mathrm{Hy}$ drogeology Journal 26(3): 945-961.

Khamforoush, M., Shams, K., Thovert, J. F. and Adler, P. M. (2008). Permeability and percolation of anisotropic three-dimensional fracture networks, Physical Review E - Statistical, Nonlinear, and Soft Matter Physics 77(5): 1-10.

Kordilla, J., Noffz, T., Dentz, M., Geyer, T. and Tartakovsky, A. M. (2017). Effect of unsaturated flow modes on partitioning dynamics of gravity-driven flow at a simple fracture intersection: laboratory study and three-dimensional smoothed particle hydrodynamics simulations., Water Resources Research 53(11): 9496-9518.

Kordilla, J., Sauter, M., Reimann, T. and Geyer, T. (2012). Simulation of saturated and unsaturated flow in karst systems at catchment scale using a double continuum approach, Hydrology and Earth System Sciences 16(10): 3909-3923.

Kordilla J., Tartakovsky A.M., G. T. (2013). A smoothed particle hydrodynamics model for droplet and film flow on smooth and rough fracture surfaces, Advances in Water Resources 59: 1-14.

Li, J., Cherubini, C., Galindo Torres, S. A., Li, Z., Pastore, N. and Li, L. (2018). Laboratory investigation of flow paths in $3 \mathrm{~d}$ self-affine fractures with lattice boltzmann simulations, Energies 11(1): 168.

Libersky, L. D., Petschek, A. G., Carney, T. C., Hipp, J. R. and Allahdadi, F. A. (1993). High Strain Lagrangian Hydrodynamics.

Liu, H. H., Doughty, C. and Bodvarsson, G. S. (1998). An active fracture model for unsaturated flow and transport in fractured rocks, Water Resources Research 34(10): 2633-2646.

Liu, H. H., Zhang, G. and Bodvarsson, G. S. (2003). The Active Fracture Model: Its Relation to Fractal Flow Patterns and an Evaluation Using Field Observations, Vadose Zone Journal 2: 259-269.

Morris, J. P., Fox, P. J. and Zhu, Y. (1997). Modeling low Reynolds number incompressible flows using SPH, Journal of Computational Physics 136(1): 214-226. 
Mourzenko, V. V., Thovert, J. F. and Adler, P. M. (2004). Macroscopic permeability of three-dimensional fracture networks with power-law size distribution, Physical Review E - Statistical Physics, Plasmas, Fluids, and Related Interdisciplinary Topics 69(6): 13.

Nicholl, M., Glass, R. and Wheatcraft, S. (1994). Gravity-driven infiltration instability in initially dry nonhorizontal fractures, Water Resources Research 30(9): 2533-2546.

Nicholl, M. J. and Glass, R. J. (2005). Infiltration into an analog fracture: Experimental observations of gravity-driven fingering, Vadose Zone Journal 4: 1123-1151.

Nicholl, M. J., Rajaram, H., Glass, R. J. and Detwiler, R. (1999). Saturated flow in a single fracture: Evaluation of the Reynolds equation in measured aperture fields, Water Resources Research 35(11): 3361-3373.

Nimmo, J. R. (2010). Theory for source-responsive and free-surface film modeling of unsaturated flow, Vadose Zone Journal 9: 295-306.

Nimmo, J. R. (2012). Preferential flow occurs in unsaturated conditions, Hydrological Processes 26(5): 786-789.

Noffz, T., Dentz, M. and Kordilla, J. (2018). Analogue fracture experiments and analytical modeling of unsaturated percolation dynamics in fracture cascades, Vadose Zone Journal .

Patriarche, D., Pili, E., Adler, P. M. and Thovert, J. F. (2007). Stereological analysis of fractures in the Roselend tunnel and permeability determination, Water Resources Research 43(9): 1-13.

Plimpton, S. (1995). Fast parallel algorithms for short-range molecular dynamics, Computational Physics 117(1): 1-19.

Ponson, L., Bonamy, D. and Bouchaud, E. (2006). Two-dimensional scaling properties of experimental fracture surfaces, Physical Review Letters 96(3): 035506.

Richards, L. A. (1931). Capillary conduction of liquids through porous mediums, Physics 1(5): 318-333.

Sahimi, M. (2011). Flow and transport in porous media and fractured rock: from classical methods to modern approaches, John Wiley $\backslash \&$ Sons.

Shigorina, E., Kordilla, J. and Tartakovsky, A. M. (2017). Smoothed particle hydrodynamics study of the roughness effect on contact angle and droplet flow, Physical Review E 96(3): 033115.

Snow, D. (1996). Rock fracture spacings, openings and porosities, J. Soil Mech. Found. Div. 95(SM3): 880-883.

Tartakovsky, A., Ferris, K. F. and Meakin, P. (2009). Lagrangian particle model for multiphase flows, Computer Physics Communications 180(10): 1874-1881.

Tartakovsky, A. M. and Panchenko, A. (2016). Pairwise force smoothed particle hydrodynamics model for multiphase flow: surface tension and contact line dynamics, Journal of Computational Physics 305: 1119-1146.

Tartakovsky, A. and Meakin, P. (2005a). Modeling of surface tension and contact angles with smoothed particle hydrodynamics, Physical Review E 72(2). 
Tartakovsky, A. and Meakin, P. (2005b). Simulation of unsaturated flow in complex fractures using smoothed particle hydrodynamics, Vadose Zone Journal 4(3): 848855.

Tartakovsky, A. and Meakin, P. (2006). Pore scale modeling of immiscible and miscible fluid flows using smoothed particle hydrodynamics, Advances in Water Resources 29(10): 1464-1478.

Therrien, R. and Sudicky, E. (1996a). Three-dimensional analysis of variablysaturated flow and solute transport in discretely-fractured porous media, Journal of Contaminant Hydrology 23(1-2): 1-44.

Therrien, R. and Sudicky, E. (1996b). Three-dimensional analysis of variablysaturated flow and solute transport in discretely-fractured porous media, Journal of Contaminant Hydrology 23(1-2): 1-44.

Tokunaga, T. K. and Wan, J. (1997). Water film flow along fracture surfaces of porous rock, Water Resources Research 33.

Tokunaga, T. K. and Wan, J. (2001). Surface-zone flow along unsaturated rock fractures, Water Resources Research 37(2): 287-296.

Wang, J. S. Y. and Narasimhan, T. N. (1985). Hydrologic Mechanisms Governing Fluid Flow in a Partially Saturated, Fractured, Porous Medium, Water Resources Research 21(12): 1861-1874.

URL: $h$ ttp://doi.wiley.com/10.1029/WR021i012p01861

Wang, L., Cardenas, M. B., Slottke, D. T., Ketcham, R. A. and Sharp, J. M. (2015). Modification of the Local Cubic Law of fracture flow for weak inertia, tortuosity, and roughness, Water Resources Research 51(4): 2064-2080.

URL: $h t t p: / /$ doi.wiley.com/10.1002/2014WR015815

Wang, M., Chen, Y.-F., Ma, G.-W., Zhou, J.-Q. and Zhou, C.-B. (2016). Influence of surface roughness on nonlinear flow behaviors in $3 \mathrm{~d}$ self-affine rough fractures: Lattice boltzmann simulations, Advances in Water Resources 96: 373-388.

Wang, Z., Tuli, A. and Jury, W. A. (2003). Unstable flow during redistribution in homogeneous soil, Vadose Zone Journal 2(1): 52-60.

Wendland, H. (1995). Piecewise polynomial, positive definite and compactly supported radial functions of minimal degree., Advances in Computational Mathematics 4(1): 389-396.

Wu, Y.-S., Liu, H. H. and Bodvarsson, G. S. (2004). A triple-continuum approach for modeling flow and transport processes in fractured rock, Journal of Contaminant Hydrology 73(1-4): 145-179.

Zhu, Y. I., Fox, P. J. and Morris, J. P. (1999). A pore-scale numerical model for flow through porous media, International Journal for Numerical and Analytical Methods in Geomechanics 23: 881-904. 


\title{
Chapter 6
}

\section{Multiscale Smoothed Particle Hydrodynamics model development for simulating preferential flow dynamics in fractured porous media ${ }^{3}$}

\begin{abstract}
.
Here, we present our newly developed multiscale pairwise-force smoothed particle hydrodynamics (PF-SPH) model for the characterization of flow in fracturedporous media. The fully-coupled multiscale PF-SPH model is able to simulate flow dynamics within a porous and permeable matrix governed by the volume-effective Richards equation coupled to the discretely resolved Lagrangian flow dynamics within adjacent fractures based on the Navier-Stokes equation. Flow from fracture to the the porous matrix is modeled by an efficient particle removal algorithm and a virtual water redistribution formulation in order to enforce mass and momentum conservation. The model is validated via (1) comparison to a FEM model (COMSOL) for the Richards-based flow dynamics in a partially saturated medium and (2) laboratory experiments to cover more complex cases of free-surface flow dynamics and imbibition into the porous matrix. For the laboratory experiments, a Seeberger sandstone is used due to its well known homogeneous pore space properties. The saturated hydraulic conductivity of the permeable matrix is estimated from a pore size and grain size distribution analysis. The developed PF-SPH model shows a good correlation with a COMSOL model and all types of laboratory experiments.

We employ the proposed model to study preferential flow dynamics for different infiltration rates. Under the term "preferential flow" we consider a vertical fracture flow, which provides a rapid water transmission in contrast to a diffuse/ porousmedium flow. Depending on an infiltration rate and water inlet location, preferential/fracture flow can occur immediately or with a delay. In case of a delay, water accumulates on the surface first (ponding), then a fracture rapidly transmits water. For the immediate fracture flow, ponding occurs only when a fracture space is fully saturated with water. For all these cases, a preferential flow is much faster than a diffuse flow even under unsaturated conditions.

Finally, we study the infiltration dynamics in rough fractures adjacent to an impermeable or permeable matrix for different infiltration rates. The simulation results

\footnotetext{
${ }^{3}$ Shigorina, E., Rüdiger, F., Tartakovsky, A. M., Sauter, M., and Kordilla, J. (2020): Multiscale smoothed particle hydrodynamics model development for simulating preferential flow dynamics in fractured porous media. Water Resources Research, 2020WR027323, under revision
} 
show a significant delay in arrival times for small infiltration rates when a permeable porous matrix is employed, as opposed to an impermeable one. In contrast, for higher infiltration rates, water rapidly flows through the fracture to the system outlet without any significant delay in arrival time even in the presence of the permeable matrix. The analysis of the amount of water stored in permeable fracture walls and in a fracture void space shows that for the small infiltration rate, most of the injected water is sucked into the porous matrix. For the large infiltration rate, the flow velocity is higher and most of the water flows rapidly to the bottom of the fracture without an intensive matrix saturation.

\subsection{Introduction}

Flow in partially saturated porous media is commonly described by the volumeaveraged Richards (1931) equation. While it was originally developed for soil systems, the Richards equation is often applied to model flow in fractured systems (Heilweil et al.; 2015; Therrien and Sudicky; 1996), when the fracture density is sufficiently high (or fracture apertures are rather small) and an REV can be defined. Given the complexity of gravity-driven flows, many discrete flow and transport processes, including fingering, preferential flow pathway formation, meandering, and erratic flow mode dynamics (droplets, rivulets), cannot be described properly described by the Richards equation.

Preferential flow within the unsaturated (vadose) zone are known to strongly influence groundwater recharge, infiltration and contaminant transport (Nimmo; 2010). There is no clear answer in the literature under which condition preferential flow occurs and what are the main parameters influencing the preferential flow dynamics. Some authors (Buscheck et al.; 1991; Nitao; 1991) provide a criteria for a critical flux under which preferential flow occurs. They assert, that for infiltration rates smaller than the critical flux, a diffuse/porous-medium flow dominates in the system. For fluxes larger than the critical flux, fracture flow dominates.

Another authors (Germann et al.; 2007; Nimmo; 2010, 2012) provide examples demonstrating, that preferential flow occurs under various unsaturated and partially saturated conditions, without surface ponding or full matrix saturation, and in the absence of hydraulic equilibrium. Preferential flow also creates a nonuniform water distribution on a surface, which affects an infiltration event (DiCarlo et al.; 1999).

Hence, unsaturated flow in fractures is not well understood due to uncertainty in generalizing flow processes, scale effects, characterization of process parameters across scales, and the assessment of their relevance in the prediction of large scale problems, e.g. the regional hydraulics of fault zones.

We use the proposed multiscale model to study conditions leading to preferential flow. The pairwise-force smoothed particle hydrodynamics (PF-SPH) component of the multiscale model is implemented in LAMMPS (Kordilla et al.; 2017; Plimpton; 1995). It is based on PF-SPH discretization of the Navier-Stokes equations and can efficiently model flow through fractures or fracture networks and adequately recover all relevant flow dynamics including the effects of free surfaces and surface tension (Kordilla et al.; 2017; Kordilla J.; 2013; Shigorina et al.; 2017, 2019). However, in porous-fractured systems, the porous and/or permeable matrix represents an important storage compartment and influences flow dynamics within the highly permeable fractures. 
In this work, we present a fully-coupled multiscale PF-SPH model, which is able to simulate free-surface flow within fractures using a discretization of the NavierStokes (NS) equation coupled to a SPH discretization of the Richards equation (Richards; 1931). The PF-SPH-LAMMPS code has been extensively validated (Kordilla et al.; 2017; Kordilla J.; 2013; Shigorina et al.; 2017) for simulating gravity-driven freesurface and fracture flows under dynamic wetting conditions. The newly developed code for simulating flow in porous media and at a fracture-matrix interface is validated against a finite-element COMSOL model and small-scale laboratory experiments.

In order to study the preferential flow dynamics, we investigate at which infiltration rates a fracture flow dominates, and at which rates a fracture acts as a flow barrier and ponding occurs, and, as a result, a diffuse flow dominates in the system. Finally, we study the influence of fracture wall permeability and storage properties of the porous matrix on the arrival times for different infiltration rates. We consider two types of rough fractures: (1) with permeable and (2) impermeable adjacent matrix. Each fracture has two surfaces with a width of $5.0 \mathrm{~cm}$, a length of $10.0 \mathrm{~cm}$, and a thickness of $1.0 \mathrm{~cm}$, separated by $2.0 \mathrm{~mm}$ aperture. The fracture roughness is characterized by the Hurst exponent $\zeta$ (Bouchaud et al.; 1990; Shigorina et al.; 2019) and an initial maximum value $\Delta$ for the random displacement from a planar surface.

\subsection{Governing Equations and the PF-SPH method}

In the following, we introduce the governing partial differential equations (PDEs) for the studied system and give an overview of the employed SPH model, including SPH discretization of the PDEs and boundary conditions, as well as the coupling procedure between the NS and Richards domains.

Partially saturated flow in porous media is commonly modeled using the Richards equation and suitable pressure-saturation relationships:

$$
\frac{\partial \Theta(\psi)}{\partial t}=\left(C_{m}+\rho \mathbf{g} S_{e} S_{s}\right) \frac{\partial \psi}{\partial t}=\nabla \cdot \mathbf{K}_{s} k_{r}(\psi) \nabla \psi+\frac{\partial K(\psi)}{\partial z},
$$

where $\Theta$ is the water content, $\psi$ is the hydraulic head, $\mathbf{K}_{s}$ is the saturated hydraulic conductivity, $S_{s}$ is the specific storage coefficient, $\rho$ is the water density, and $\mathbf{g}$ is the gravitational acceleration. The parameters $C_{m}$ (specific moisture capacity), $S_{e}$ (effective saturation), and $k_{r}$ (relative hydraulic conductivity) are found from the van Genuchten relationships (Van Genuchten; 1980):

$$
\begin{aligned}
& S e=\left\{\begin{array}{ll}
\frac{1}{\left[1+|\alpha \psi|^{n}\right]^{m}} & \text { if } \psi<0 \\
1 & \text { if } \psi \geq 0
\end{array},\right. \\
& k_{r}=\left\{\begin{array}{ll}
S e^{0.5}\left[1-\left(1-S e^{\frac{1}{m}}\right)^{m}\right]^{2} & \text { if } \psi<0 \\
1 & \text { if } \psi \geq 0
\end{array},\right. \\
& C_{m}=\left\{\begin{array}{ll}
\frac{\alpha m}{1-m}\left(\Theta_{S}-\Theta_{r}\right) S e^{\frac{1}{m}}\left(1-S e^{\frac{1}{m}}\right)^{m} & \text { if } \psi<0 \\
0 & \text { if } \psi \geq 0
\end{array} .\right.
\end{aligned}
$$


Here, $\alpha$ and $n$ are the van Genuchten parameters, $m=1-1 / n$, and $\Theta_{s}$ and $\Theta_{r}$ are the saturated and residual liquid volume fractions respectively.

The free-surface fracture flow is governed by the continuity equation,

$$
\frac{d \rho}{d t}=-\rho(\nabla \cdot \mathbf{v})
$$

and the momentum conservation equation,

$$
\frac{d \mathbf{v}}{d t}=-\frac{1}{\rho} \nabla P+\frac{\mu}{\rho} \nabla^{2} \mathbf{v}+\mathbf{g} .
$$

At the water-air interface the Young-Laplace boundary condition

$$
P \mathbf{n}=\boldsymbol{\tau}_{w} \cdot \mathbf{n}+S \sigma \mathbf{n},
$$

and the continuity condition

$$
\left(\mathbf{v}-\mathbf{v}_{b}\right) \cdot \mathbf{n}=0
$$

are enforced.

The contact angle is prescribed at the water-air-solid contact line and the no-slip boundary condition at the boundary between water and solid phases. Here, $\boldsymbol{\tau}_{w}=$ $\left[\mu\left(\nabla \mathbf{v}+\nabla \mathbf{v}^{\mathrm{T}}\right)\right]$ is the viscous stress tensor, $\mathbf{v}$ the fluid velocity, $\mathbf{v}_{b}$ is the boundary velocity, $P$ is the pressure, $\mu$ the viscosity, $\mathbf{n}$ is the normal vector pointing away from the non-wetting phase, and $\mathbf{g}$ the gravitational acceleration.

To numerically solve these equations with the SPH method, we discretize the porous matrix with a set of solid particles and fluid in the fracture with a set of fluid particles. The positions of solid particles are fixed and their velocities are set to zero. The positions and velocities of fluid particles are found from the momentum conservation equation discretized with the weakly compressible pairwise SPH scheme (Kordilla et al.; 2017; Kordilla J.; 2013; Morris et al.; 1997; Tartakovsky and Meakin; 2005b):

$$
\begin{aligned}
& \frac{d \mathbf{v}_{i}}{d t}=-\sum_{j=1}^{N} m_{j}\left(\frac{P_{j}}{\rho_{j}^{2}}+\frac{P_{i}}{\rho_{i}^{2}}\right) \frac{\mathbf{r}_{i j}}{r_{i j}} \cdot \frac{d W\left(r_{i j}, h\right)}{d r_{i j}}+ \\
& 2 \mu \sum_{j=1}^{N} m_{j} \frac{\mathbf{v}_{i j}}{\rho_{i} \rho_{j} r_{i j}} \cdot \frac{d W\left(r_{i j}, h\right)}{d r_{i j}}+\mathbf{g}+\frac{1}{m_{i}} \sum_{j=1}^{N} \mathbf{F}_{i j},
\end{aligned}
$$

and

$$
\frac{d \mathbf{r}_{i}}{d t}=\mathbf{v}_{i},
$$

where the summation is performed over all particles including fluid and solid particles. In Eqs. (6.15)-(6.8), $\mathbf{r}_{i j}=\mathbf{r}_{i}-\mathbf{r}_{j}$ and $r_{i j}=\left|\mathbf{r}_{i}-\mathbf{r}_{j}\right|, m_{i}=m_{j}=m_{0}$ is the (constant) mass of particle $i$ and $j, \rho_{j}$ and $P_{j}$ are the density and pressure of fluid carried by particle $j$, and $h$ is the support range of the kernel $W$. Fluid and solid particles are assumed to have the same mass and $\rho_{i}$ is computed for both fluid and solid particles as (Morris et al.; 1997; Tartakovsky and Meakin; 2005b)

$$
\rho_{i}=\sum_{j=1}^{N} m_{j} W\left(\mathbf{r}_{i j}, h\right),
$$


The pressure of both fluid and solid particles is computed from the equations of state Batchelor (1967):

$$
P_{i}=P_{0}\left\{\left(\frac{\rho_{i}}{\rho_{0}}\right)^{\gamma}-1\right\}
$$

where

$$
P_{0}=\frac{c^{2} \rho_{0}}{\gamma}
$$

$\gamma=7, \rho_{0}$ is the equilibrium particle density, and the speed of sound $c$ is chosen such that the relative density fluctuation $|\delta \rho| / \rho$ is small (less than $3 \%$ ) to approximate an incompressible fluid.

In Eqs. (6.15), (6.7), and (6.9), we use $W$ in the form of a so-called "Wendland" kernel (Wendland; 1995):

$$
W=\alpha_{k}\left\{\begin{array}{ll}
\left(1-\frac{|\mathbf{r}|}{h}\right)^{3} & \text { if } 0 \leq|\mathbf{r}|<h \\
0 & \text { if }|\mathbf{r}| \geq h
\end{array},\right.
$$

where $\alpha_{k}=168 / 16 \pi h^{3}$.

The force $\mathbf{F}_{i j}$ in Eq. (6.7) is used to impose the Young-Laplace boundary condition. Following Tartakovsky and Meakin (2005b), Tartakovsky and Panchenko (2016), Kordilla J. (2013) and Kordilla et al. (2017) we employ a combination of kernel functions to generate a continuous function with short-range repulsive and longrange attractive components:

$$
\mathbf{F}_{i j}=s_{i j} \begin{cases}\left(\tilde{A} \tilde{W}\left(r_{i j}, h_{1}\right) \frac{\mathbf{r}_{i j}}{r_{i j}}+\tilde{B} \tilde{W}\left(r_{i j}, h_{2}\right) \frac{\mathbf{r}_{i j}}{r_{i j}}\right) & \text { if } \mathbf{r}_{i j} \leq h \\ 0 & \text { if } \mathbf{r}_{i j}>h,\end{cases}
$$

where $\tilde{W}$ is the cubic spline function

$$
\tilde{W}\left(r_{i j}, h\right)= \begin{cases}1-\frac{3}{2}\left(\frac{\mathbf{r}_{i j}}{h}\right)^{2}+\frac{3}{4}\left(\frac{\mathbf{r}_{i j}}{h}\right)^{3} & \text { if } 0 \leq \frac{\mathbf{r}}{h}<0.5 \\ \frac{1}{4}\left(2-\frac{\mathbf{r}_{i j}}{h}\right)^{3} & \text { if } 0.5 \leq \frac{\mathbf{r}_{i j}}{h}<1 \\ 0 & \text { if } \frac{\mathbf{r}_{i j}}{h} \leq 1 .\end{cases}
$$

Here, $\tilde{A}, \tilde{B}, h_{1}$, and $h_{2}$ determine the shape of $\mathbf{F}_{i j}$. We set $\tilde{A}=8, \tilde{B}=-1$, and $h_{1}=0.5$, $h_{2}=1$. For a given $\mathbf{F}_{i j}$ shape, $s_{i j}$ determines the magnitude of surface tension and static contact angle.

The parameter $s_{i j}$ is equal to $s_{f f}$ for the interaction between two fluid particles and $s_{s f}$ for the interaction between fluid and solid particles. The ratio of $s_{f f}$ and $s_{s f}$ controls the static and dynamic contact angles. For a liquid to wet the surface, $s_{f f}$ should be set greater than $s_{s f}$ and vice versa.

The SPH discretization of Eqs. (6.1)-(6.2) is:

$$
\frac{d \Theta_{i}}{d t}=\left(C_{m_{i}}+\rho_{i} \mathbf{g} S e_{i} S_{i}\right) \frac{d \psi_{i}}{d t}=\sum_{j=1}^{N} 2 \frac{m_{i} m_{j}}{m_{i}+m_{j}} \frac{\rho_{i}+\rho_{j}}{\rho_{i} \rho_{j}} \cdot \mathbf{K}_{s} k_{r_{i}}\left(d \psi_{i j}+d z_{i j}\right) \cdot \frac{d W\left(r_{i j}, h\right)}{d r_{i j}} .
$$

Here, each particle (solid and fluid) is assigned an initial water content $\Theta$ and initial hydraulic head $\psi$. The water content of a solid particle is defined as the volume 
of water in the particle divided by the volume of this particle. The water content of fluid particles is defined as the volume of fluid carried by the particle divided by its initial volume.

The fluid particles are initially fully saturated and are assigned $\Theta_{f}=1.0$ and $\psi_{f}=0.0 \mathrm{~m}$. Depending on the type of a problem, the solid particles are assigned $\Theta_{b}=0.0$ or $\Theta_{b}$ equal to a residual water content. Once fluid particles come into a contact with solid particles the exchange of fluid is governed by the Richards equation, i.e. a pressure-head dependent transfer is established. The changes in water content and pressure head for solid and fluid particles are found from Eq. (6.15). The maximum $\Theta_{b}$ of solid particles is equal to the saturated water content of the porous matrix based on the user defined porosity.

If the water content $\Theta_{f}$ of fluid particles falls below a critical threshold $\Theta_{f}<0.99$, we redistribute the total water content of all particles below the threshold such that most particles are fully saturated again with $\Theta_{f}=1$.0. Fluid particles that are still below the critical threshold after the redistribution are marked and removed at the end of the time step (6.1). The residual water content (commonly less than the water content of one single particle) is stored and taken into account during the next time step. This procedure is carried out over all particles within a single MPI domain.

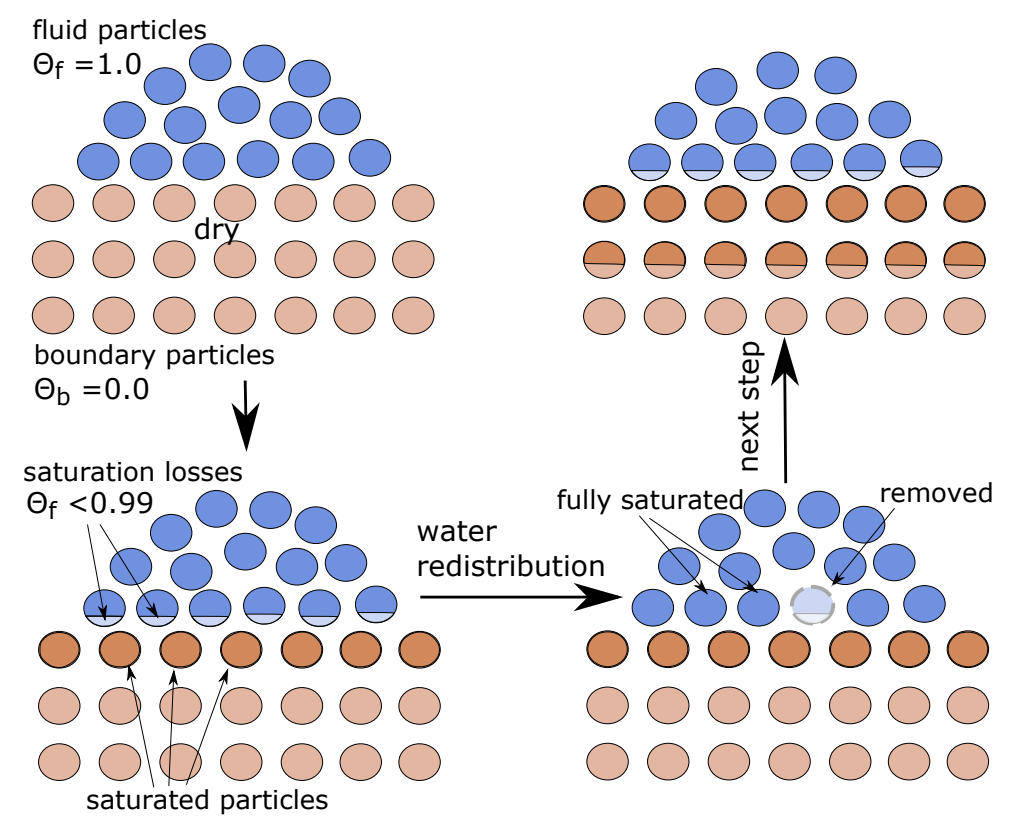

FIGURE 6.1: Particle removal algorithm.

To properly conserve the water balance in the system, we rely on the mass conservation equation:

$$
\frac{\partial \Theta}{\partial t}=\nabla \cdot\left(\sum q_{\text {in }}-\sum q_{\text {out }}\right)=0,
$$

where $q$ is the specific flux. Every time step we calculate the sum of $\Theta_{f}$ and $\Theta_{b}$ of all fluid and boundary particles based on Eq. (6.17). To control the water balance in the system, the total $\Theta$ must stay constant:

$$
\Theta=\sum \Theta_{f}+\sum \Theta_{b}=\text { const. }
$$


We employ a modified Velocity Verlet time stepping scheme (Ganzenmüller et al.; 2011):

$$
\begin{aligned}
\mathbf{v}_{i}\left(t+\frac{1}{2} \Delta t\right) & =\mathbf{v}_{i}+\frac{1}{2} \mathbf{a}_{i}(t) \\
\overline{\mathbf{v}}_{i}(t+\Delta t) & =\mathbf{v}_{i}(t)+\Delta t \mathbf{a}_{i} \\
\mathbf{r}_{i}(t+\Delta t) & =\mathbf{r}_{i}(t)+\Delta t \mathbf{v}_{i}\left(t+\frac{1}{2} \Delta t\right) \\
\mathbf{v}_{i}(t+\Delta t) & =\mathbf{v}_{i}\left(t+\frac{1}{2} \Delta t\right)+\frac{1}{2} \mathbf{a}_{i}(t+\Delta t),
\end{aligned}
$$

where the new particle acceleration $\mathbf{a}_{i}(t+\Delta t)$ can be obtained using an extrapolated velocity $\overline{\mathbf{v}}_{i}$.

Time step constraints are given by Tartakovsky and Meakin (2005b):

$$
\begin{aligned}
& \Delta t \leq 0.25 h / 3 c \\
& \Delta t \leq 0.25 \min \left(h / 3\left|\mathbf{a}_{i}\right|\right)^{1 / 2} \\
& \Delta t \leq \min \left(\rho_{i} h^{2} / 9 \mu_{i}\right)
\end{aligned}
$$

where $\left|\mathbf{a}_{i}\right|$ is the magnitude of acceleration $\mathbf{a}_{i}$.

\subsection{Model Validation}

\subsubsection{Constant pressure head boundary}

Here we provide a validation of the SPH discretization of the Richards equation under static conditions. We model a pressure head distribution inside a vertical porous column with a constant pressure head boundary. The dimensions of the column are $0.5 \times 0.5 \times 2 \mathrm{~m}$. This model setup includes 38720 solid particles, with initial pressure head $\psi_{0}=-2.0 \mathrm{~m}$, isotropic conductivity $\mathbf{K}_{s}=1 \times 10^{-4} \mathrm{~m} \mathrm{~s}^{-1}, S_{s}=$ $7.5 \times 10^{-5} \mathrm{~Pa}^{-1}, \Theta_{s}=0.25, \Theta_{r}=0.0$, and the van Genuchten parameters $n=2$, $m=0.5, \alpha=1$ (Fig. 6.2a). A constant pressure head boundary with $\psi_{b}=-0.5 \mathrm{~m}$ is prescribed at the bottom of the domain. The particles are placed on a uniform cubic lattice with a lattice size of $\Delta x=2.5 \times 10^{-2} \mathrm{~m}$. The mass and density of each particle is $m_{0}=1 \times 10^{-3} \mathrm{~kg}, \rho_{0}=1000 \mathrm{~kg} \mathrm{~m}^{-3}$, respectively. The smoothing length is set to $h=8.55 \times 10^{-2} \mathrm{~m}$. This yields an average number of 40 interacting particles, which was shown to be sufficient to achieve an accurate solution (Kordilla et al.; 2017; Kordilla J.; 2013; Tartakovsky and Meakin; 2005a). Figure 6.2 shows the SPH simulation results for the pressure head inside the vertical column at $0,1,6$, and 16 hours.

The validation is carried out via comparison with a FEM COMSOL model. Figure 6.3 shows the pressure head distributions along the vertical column at 1, 6, and 16 hours for our SPH and the COMSOL model. To quantify the difference in the SPH and COMSOL pressure head solutions, for $t=1,6$, and 16 hours we calculate the standard deviation

$$
s_{t}=\sqrt{\frac{\sum_{i=1}^{N}\left(\psi_{z_{i}}^{s}-\psi_{z_{i}}^{c}\right)^{2}}{N-1}}
$$

and standard error

$$
S E_{t}=\frac{S_{t}}{\sqrt{N}}
$$

where $\psi_{z}^{s}$ and $\psi_{z}^{c}$ are the SPH and COMSOL pressure head solutions at distances $z=0.0,0.25,0.5,0.75,1.0,1.25,1.5,1.75$, and $2.0 \mathrm{~m}$, and the number of measurements 


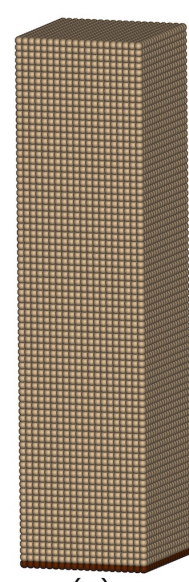

(a)

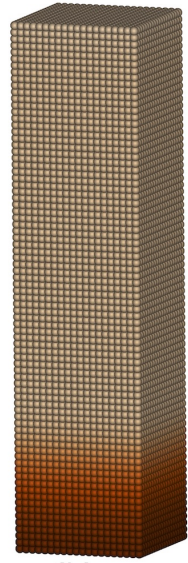

(b)

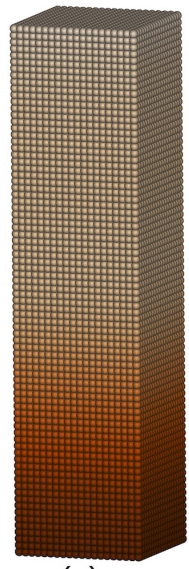

(c)

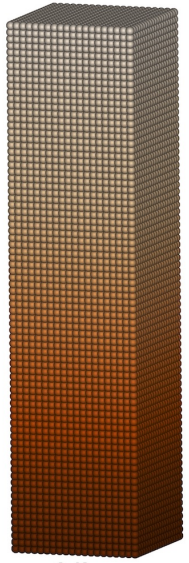

(d)
Pressure head $(\mathrm{m})$

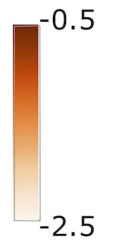

$-2.5$

FIGURE 6.2: Pressure head distributions for a vertical column with constant pressure head boundary at different times: (a) $t_{0}=0 \mathrm{~h}$, (b)

$$
t_{1}=1 \mathrm{~h},(\mathrm{c}) t_{2}=6 \mathrm{~h} \text {, and (d) } t_{3}=16 \mathrm{~h} \text {. }
$$

is $N=9$. Table 6.1 provides standard deviations and standard errors for $t=1,6$, and 16 hours. The average standard deviation is $\tilde{s_{t}}=3.9 \times 10^{-2} \mathrm{~m}$, and the average standard error is $S \tilde{E}_{t}=1.0 \times 10^{-2} \mathrm{~m}$, which is less than the particle spacing $\Delta x$ and indicates excellent numerical accuracy of the SPH model.

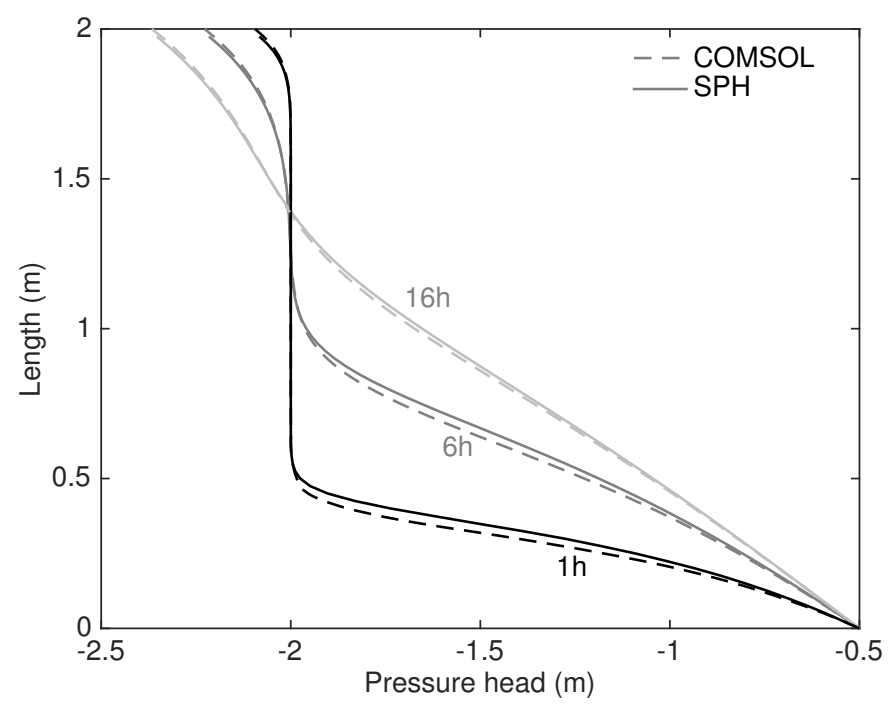

FIGURE 6.3: Comparison of pressure heads at different times for $\mathrm{SPH}$ and COMSOL models.

Next, we compare our model with three laboratory experiments, including fluid resting on a porous permeable surface or flowing over it.

\subsubsection{Drop imbibition}

\section{Experimental and simulation setup}

First, we study the infiltration of a droplet into a sandstone. In the laboratory experiment, a water droplet with radius $1.8 \mathrm{~mm}$ is placed above a slice of sandstone 
TABLE 6.1: Standard deviations and standard errors of pressure heads at $t=1,6$, and 16 hours.

\begin{tabular}{ccccc}
\hline \multicolumn{2}{c}{ Standard deviation $(\mathrm{m})$} & & \multicolumn{2}{c}{ Standard Error $(\mathrm{m})$} \\
\cline { 1 - 3 } \cline { 5 - 6 }$s_{t=1 h}$ & $3.9 \times 10^{-2}$ & & $S E_{t=1 h}$ & $1.3 \times 10^{-2}$ \\
$s_{t=6 h}$ & $3.0 \times 10^{-2}$ & & $S E_{t=6 h}$ & $1.0 \times 10^{-2}$ \\
$s_{t=16 h}$ & $2.1 \times 10^{-2}$ & & $S E_{t=16 h}$ & $0.7 \times 10^{-2}$ \\
$\tilde{s_{t}}$ & $3.0 \times 10^{-2}$ & & $S \tilde{E}_{t}$ & $1.0 \times 10^{-2}$ \\
\hline
\end{tabular}

(type "Seeberger") at a distance of $5.8 \mathrm{~mm}$ between the surface and droplet center (Fig. 6.4a, top). After the droplet is released from the pipette, it comes into contact with the sandstone surface and slowly infiltrates the sandstone slice. During the experiment, the changes in droplet size and shape are observed visually and the infiltration time into the solid matrix is measured.

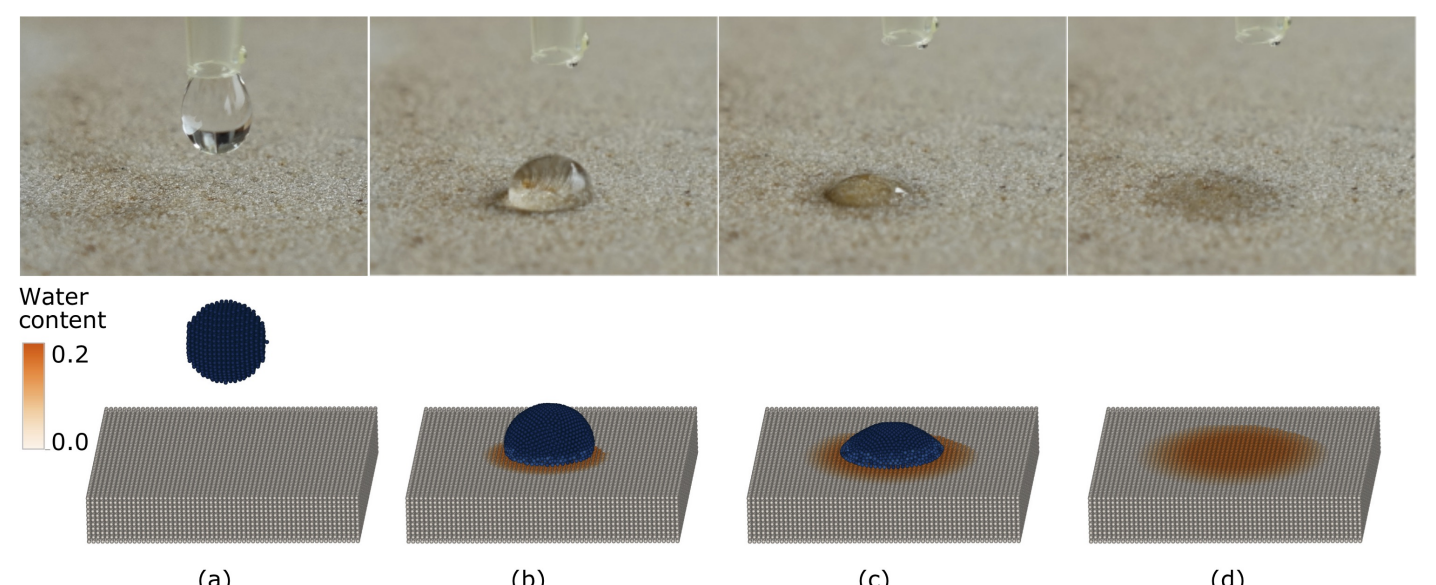

(a)

(b)

(c)

(d)

FIGURE 6.4: The experimental (top) and simulation (bottom) results of droplet imbibition at different times: (a) $t_{0}=-0.004 \mathrm{~s}$, (b) $t_{1}=$ $0.396 \mathrm{~s}$, (c) $t_{2}=1.836 \mathrm{~s}$, and (d) $t_{3}=2.676 \mathrm{~s}$.

To model this laboratory experiment, we create a rectangular block of solid particles, representing the sandstone slice, and a sphere of fluid particles at a height of $5.8 \mathrm{~mm}$ above the solid surface (Fig. $6.4 \mathrm{a}$, bottom). The dimensions of the solid block are $12 \times 12 \times 2 \mathrm{~mm}$, the water droplet has a radius $1.8 \mathrm{~mm}$.

Solid particles are placed on a uniform cubic lattice with a lattice size of $\Delta x=$ $2.0 \times 10^{-4} \mathrm{~m}$. Each particle (solid and fluid) has a density $\rho_{0}=1000 \mathrm{~kg} / \mathrm{m}^{3}$ and a mass $m_{0}=\rho_{0}(\Delta x)^{3}=8 \times 10^{-9} \mathrm{~kg}$. The viscosity is set to $\mu=1.296 \times 10^{-3} \mathrm{Pas}$, the speed of sound to $c=2.5 \mathrm{~m} / \mathrm{s}$, the gravitational acceleration to $g=9.81 \mathrm{~m} / \mathrm{s}^{2}$, and the smoothing length to $h=\sqrt[3]{40}(\Delta x)=6.84 \times 10^{-4} \mathrm{~m}$, where 40 is the particle number density, i.e. the number of interacting particles within the kernel range $h$. The system is resolved with 39600 solid and 3042 fluid particles. The input simulation parameters are: the porosity $\epsilon_{p}$, permeability $\mathbf{K}_{s}$, storage coefficient $S_{s}$, saturated $\Theta_{s}$ and residual $\Theta_{r}$ water content of the sandstone, and the van Genuchten parameters $\alpha, m, n$. These parameters can be estimated from the grain size and pore size distribution analysis of Seeberger Sandstone sample as demonstrated in the following section. 


\section{Parameter estimation}

The effective porosity $\epsilon_{p}=0.186$ of the Sandstone is found from a pore size analysis based on mercury porosimetry (Fig. 6.5, Sustrate (2017)).

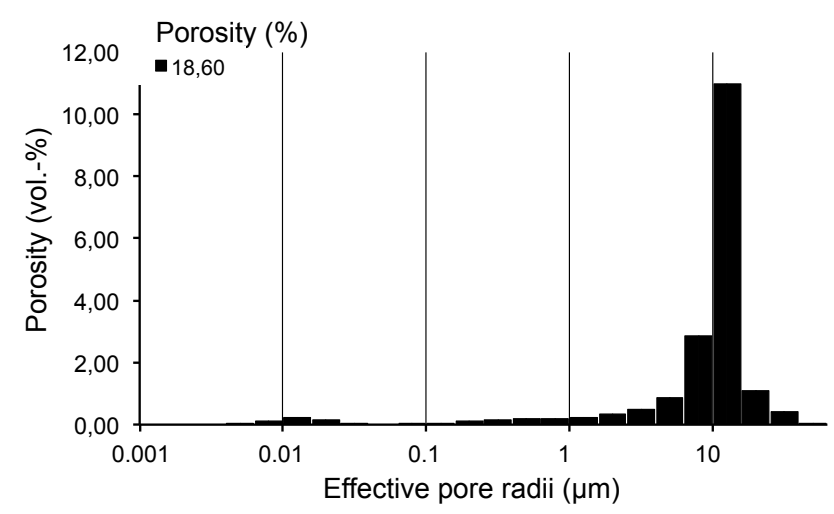

FIGURE 6.5: Porosimetry of Seeberger Sandstone (Sustrate; 2017).

The (isotropic) conductivity $\mathbf{K}_{s}$ is estimated from Kozeny-Carmen model (Carman; 1937; Kozeny; 1927):

$$
\mathbf{K}_{s}=\left(\frac{\rho g}{\mu}\right) \frac{\epsilon_{p}^{3}}{\left(1-\epsilon_{p}\right)^{2}}\left(\frac{d_{m}^{2}}{180}\right) .
$$

Based on the results of a sieve analysis (Sustrate; 2017), the representative grain size was found to be $d_{m} \approx 0.125 \mathrm{~mm}$. Together with $g=9.81 \mathrm{~m} \mathrm{~s}^{-2}$ and $\rho=$ $1000 \mathrm{~kg} \mathrm{~m}^{-3}$ the saturated hydraulic conductivity is determined with Eq. (6.22) as $\mathbf{K}_{s}=6.39 \times 10^{-6} \mathrm{~m} \mathrm{~s}^{-1}$.

The storage coefficient $S_{s}$ is found from Eq. (6.23):

$$
S_{s}=\epsilon_{p} \chi_{f}+\left(1-\epsilon_{p}\right) \chi_{p}
$$

where $\chi_{f}=4.6 \times 10^{-10} \mathrm{~Pa}^{-1}$ is the compressibility of water, $\chi_{p}=3.8 \times 10^{-6} \mathrm{~Pa}^{-1}$ is the estimated compressibility of the porous matrix based on the porosity $\epsilon_{p}=$ 0.186 (Hall et al.; 1953). Using Eq. (6.23) we obtain the storage coefficient $S_{s}=$ $3.09 \times 10^{-6} \mathrm{~Pa}^{-1}$.

The van Genuchten parameter $\alpha$ is found following Guarracino (2007) and Bear (2013):

$$
\alpha=\left(\frac{2 \sigma \cos \theta}{\rho g r_{\max }}\right)^{-1}
$$

where $\sigma=0.0735 \mathrm{~N} \mathrm{~m}^{-1}$ is the surface tension of water at $10^{\circ} \mathrm{C}, \theta=90^{\circ}$ is the static contact angle of the fluid on a solid surface, $r_{\max }=15 \mu \mathrm{m}$ is the maximum pore radius (Fig. 6.5, Sustrate (2017)), $g=9.81 \mathrm{~m} \mathrm{~s}^{-2}$, and $\rho=1000 \mathrm{~kg} \mathrm{~m}^{-3}$. Employing Eq. (6.24), we obtain $\alpha \approx 1.0 \mathrm{~m}^{-1}$. The parameters $m$ and $n=[1-m]^{-1}$ are found based on the fractal dimension $D$ (Ghanbarian-Alavijeh et al.; 2010; Mandelbrot; 1983):

$$
m=\frac{3-D}{4-D}
$$


The parameter $D$ can be found from the mass-based relationship (Boadu; 2000; Tyler and Wheatcraft; 1992):

$$
\frac{M\left(d<d_{m}\right)}{M_{T}}=\left(\frac{d_{m}}{d_{\text {max }}}\right)^{3-D},
$$

where $d_{\max }$ is the upper size limit of the particle sizes from the sieve analysis, $M_{T}$ is the total mass of a sample and $M\left(d<d_{m}\right)$ is the mass of soil with grains smaller than $d_{m}$. From the sieve analysis we obtain $M_{T}=159.0 \mathrm{~g}, M\left(d<d_{m}\right)=17.3 \mathrm{~g}, d_{m}=$ $0.125 \mathrm{~mm}$ and $d_{\max }=1.0 \mathrm{~mm}$. The parameter $D=1.93$ is estimated from Eq. (6.26) by $\log$ transforming both sides of the equation, and Eq. (6.25) yields $m \approx 0.5$, and $n=[1-m]^{-1} \approx 2.0$.

\section{Results}

During imbibition, the contact line between droplet and surface can evolve in two different ways (Lee et al.; 2016; Marmur; 1988; Siregar; 2012): (1) the contact line moves while the static contact angle remains constant, or (2) the contact line is pinned to the surface while the contact angle decreases. According to our laboratory observations, droplet imbibition into the Seeberger sandstone takes place with the pinned contact line (Fig. 6.4a-d, top). The contact angle in this case varies from $\theta=90^{\circ}$ to its minimum value close to $0^{\circ}$, while the contact line diameter stays equal to $3.9 \mathrm{~mm}$ until the droplet is completely absorbed after $2.676 \mathrm{~s}$ (Fig. 6.4, top).

Figure 6.4(bottom) shows the simulation results of the droplet imbibition at different times. Here the fluid particles are initially fully saturated and have $\Theta_{f}=1.0$ and $\psi_{f}=0.0 \mathrm{~m}$, the solid particles are initially set to $\Theta_{b}=0.01$ and $\psi_{b}=-3.8 \mathrm{~m}$ (fitted value for the given van Genuchten parameter set). The subscripts $f$ and $b$ stand for the fluid and boundary particles, respectively.

To keep the contact line pinned to the surface, after the droplet is equilibrated on the surface at $\left(t_{e q}=0.4 \mathrm{~s}\right)$, we linearly increase the interaction force $s_{s f}$ with time $t$ (Eq. 6.13) from $s_{s f}=0.0$ to $1 \times 10^{-5}$ (Fig. 6.6):

$$
s_{s f}=(4.39 t-1.60) \cdot 10^{-6},
$$

where Eq. (6.27) is empirically obtained.

The equilibrated dynamic contact angle in this case decreases from $\Theta=90^{\circ}$ to $18^{\circ}$, while the contact diameter stays equal to $3.9 \mathrm{~mm}$. The absorption time for the simulated droplet is $2.588 \mathrm{~s}$, which is close to the experimental absorption time of $2.676 \mathrm{~s}$.

\subsubsection{Water infiltration into a sandstone}

In the section, we consider the infiltration of $4.0 \mathrm{~mL}$ of water into a rectangular Seeberger sandstone sample. The dimensions of the sandstone block are $47.5 \times 8.0 \times 47.5 \mathrm{~mm}$. The back, front, left, and right sides of the sample are sealed and the water is supplied to the top of the sample. During the experiment, we measure the infiltration depth (Fig. 6.7, top) and the water level above the sandstone surface (Fig. 6.8).

In the simulation, we create a block of 280840 solid particles, which are placed on a uniform cubic lattice with the lattice size $\Delta x=4.0 \times 10^{-4} \mathrm{~m}$. A block of 62500 (equivalent to $4.0 \mathrm{~mL}$ of water) fluid particles is placed above the solid (Fig. 6.7, bottom). To reproduce the no-flow conditions at the back, front, left, and right side, we prescribe periodic boundaries to the direction of length and width of the sample. The mass and density of each solid and fluid particle is $m_{0}=6.4 \times 10^{-8} \mathrm{~kg}, \rho_{0}=$ 


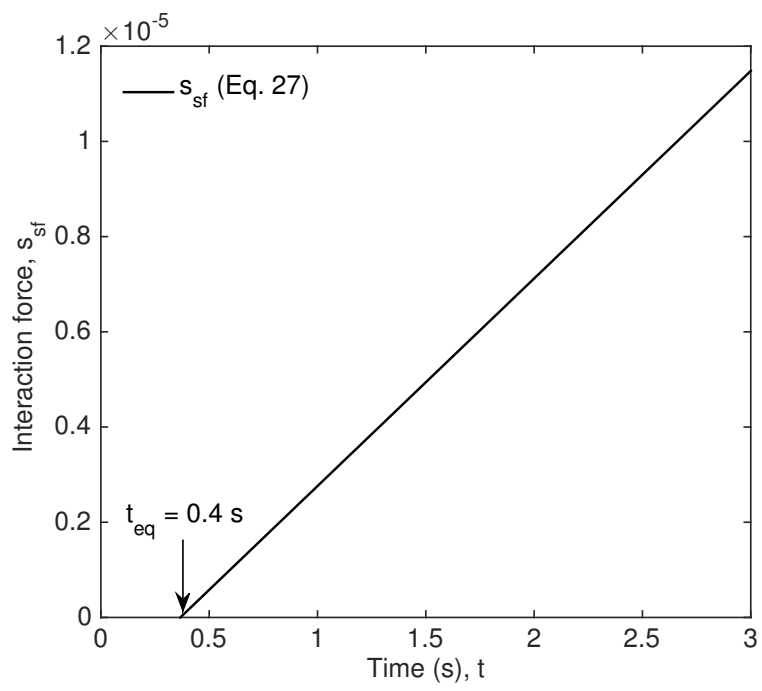

FIGURE 6.6: The dependence of interaction forces between solid and fluid particles $s_{s f}$ on time $t$, the stabilization time $t_{e q}=0.4 s$.

$1000 \mathrm{~kg} / \mathrm{m}^{3}$, respectively, and the smoothing length is set to $h=1.37 \times 10^{-3} \mathrm{~m}$. The viscosity is set to $\mu=1.296 \times 10^{-3} \mathrm{~Pa} \mathrm{~s}$, the speed of sound to $c=2.0 \mathrm{~m} / \mathrm{s}$, and the gravitational acceleration to $\mathrm{g}=9.81 \mathrm{~m} / \mathrm{s}^{2}$.

The parameters $\epsilon_{p}, \mathbf{K}_{s}, S_{s}, \psi_{f}, \psi_{b}, \Theta_{s}$ and $\Theta_{r}$, and the van Genuchten $\alpha, m, n$ are taken from the previous subsection. Figure 6.8 compares the experimental and simulation results of the decreasing water level during the infiltration into the Seeberger sandstone sample. The simulation results are in a good agreement with the laboratory experiment (Fig. 6.7, 6.8). Small deviations between simulation and experimental results are most likely due to small heterogeneities within the sandstone sample and errors in the sandstone parameters estimated in Section 6.3.2.

\subsubsection{Flow on the fracture wall and in the adjacent matrix in sandstone}

In this section, we compare the experimental and simulation results of free-surface flows on the fracture wall and in the adjacent permeable sandstone matrix and the respective discharge rates at the outlet of the fracture.

The experimental setup consists of a Seeberger sandstone sample with dimensions $47.5 \times 8.5 \times 47.5 \mathrm{~mm}$, which is placed between two acrylic glass plates. A water inlet with continuous water flux $Q=3.5 \mathrm{~mL} \mathrm{~min}^{-1}$ is located $5.0 \mathrm{~mm}$ above the upper right corner of the sandstone. A silicon rubber sheet between the acrylic glass plate and the sample prevents water flowing between the front and back side of the sample. The upper and right side surfaces are left open in order to allow freesurface films to evolve. During the experiment, the saturation of the porous matrix is observed (Fig. 6.9, top), and the water outflow mass is measured (Fig. 6.10).

In the SPH simulation, we create a block of 297381 solid particles, which are placed on a uniform cubic lattice with the lattice size $\Delta x=4.0 \times 10^{-4} \mathrm{~m}$. A certain amount of fluid particles (equivalent to the flux $Q=3.5 \mathrm{~mL} \mathrm{~min}^{-1}$ ) is added each time step to the upper right corner of the solid block within a small injection volume. The input simulation parameters are taken from the previous subsection. Figure 6.9 (bottom) shows the porous matrix saturation during the simulation. The experimental and simulation outflow mass measurements are shown in Fig. 6.10. The dotted line represents the outflow mass during the experiment, the solid straight line 


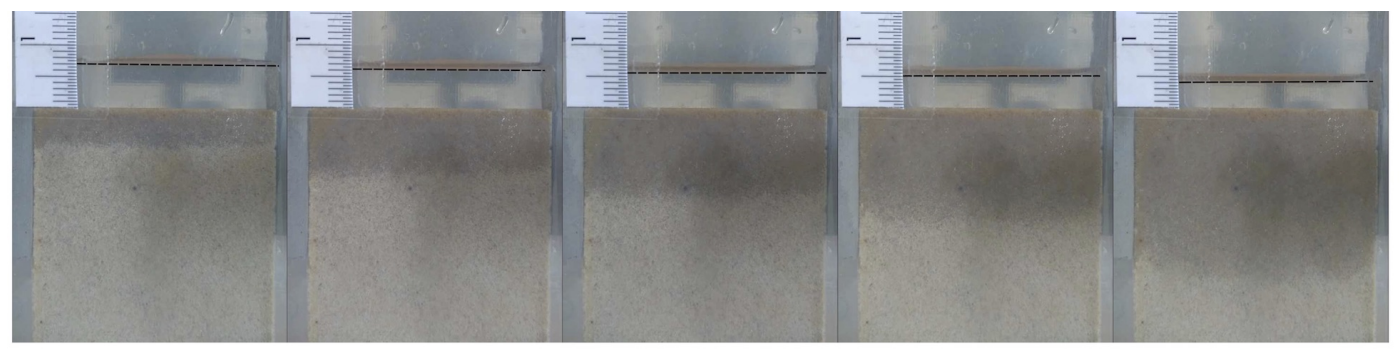

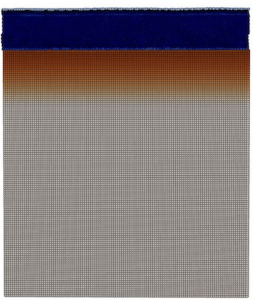

(a)

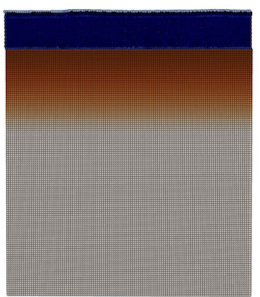

(b)

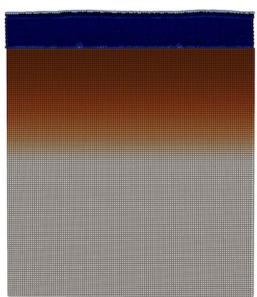

(c)

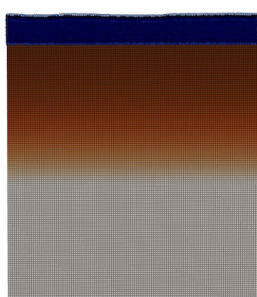

(d)

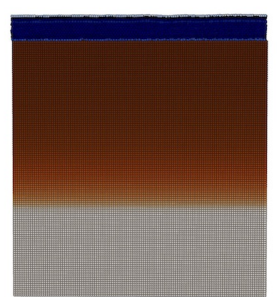

(e)

FIGURE 6.7: Comparison of the experimental (top) and simulation (bottom) results of $4.0 \mathrm{~mL}$ water infiltrating into a sandstone at different time intervals: (a) $t_{1}=3 \mathrm{~s}$; (b) $t_{2}=16 \mathrm{~s}$; (c) $t_{3}=30 \mathrm{~s}$; (d) $t_{4}=50 \mathrm{~s}$;

(e) $t_{5}=100 \mathrm{~s}$.

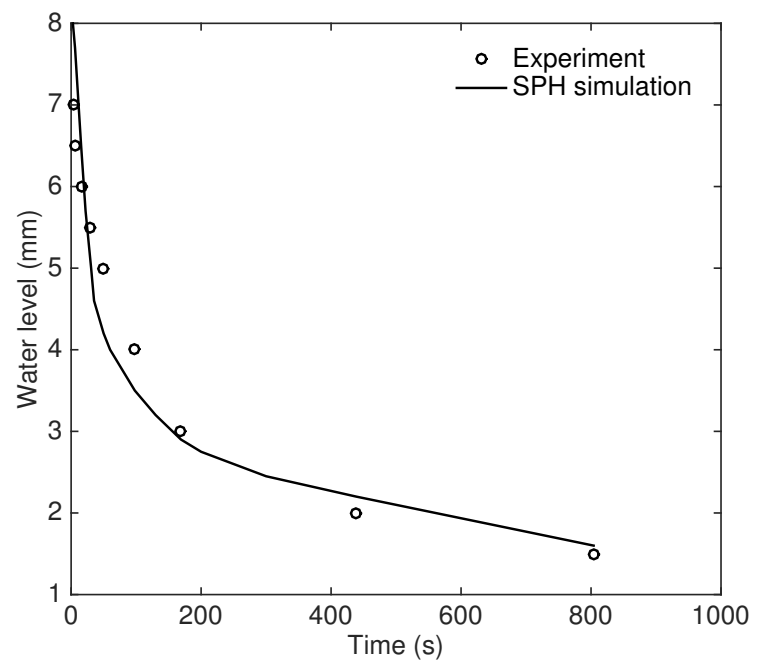

FIGURE 6.8: Experimental and simulation measurements of water level above the sandstone during infiltration.

the SPH simulation data. During the first $42 \mathrm{~s}$ of the experiment and $44 \mathrm{~s}$ of the simulation the water infiltrates into the sandstone and accumulates mostly on the top of the sample, i.e., no outflow is observed. Once the saturation of the sandstone reaches a critical threshold and enough water has accumulated on the top of the sandstone, rapid gravity-driven flow on the vertical surface is initiated and the outflow is increasing nearly linearly. At this point the system is dominated by preferential flow on the free surface and the imbibition into the porous matrix slowly diminishes. The simulation results are in a good agreement with the laboratory experiment, both in terms of the discharge rate, as well as the onset of the initial breakthrough. 


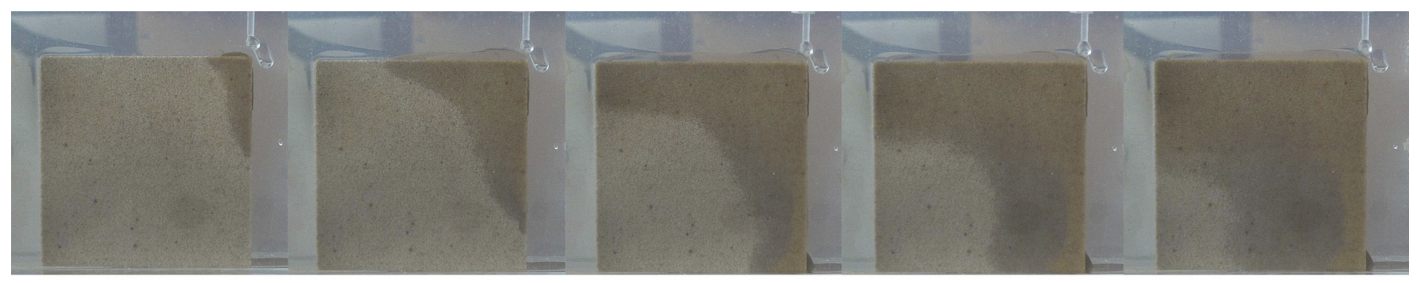

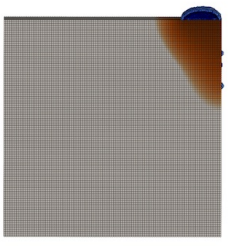

(a)

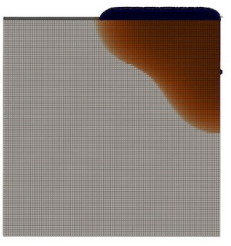

(b)

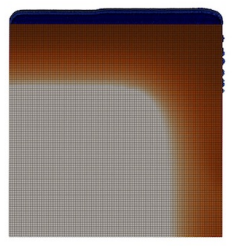

(c)

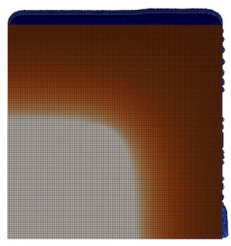

(d)

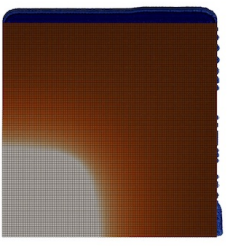

(e)

FIGURE 6.9: Comparison of experimental (top) and simulation (bottom) results of free-surface flows on a porous sandstone at different time intervals: (a) $t_{1}=5 \mathrm{~s}$; (b) $t_{2}=22 \mathrm{~s}$; (c) $t_{3}=44 \mathrm{~s}$; (d) $t_{4}=66 \mathrm{~s}$; (e) $t_{5}=110 \mathrm{~s}$.

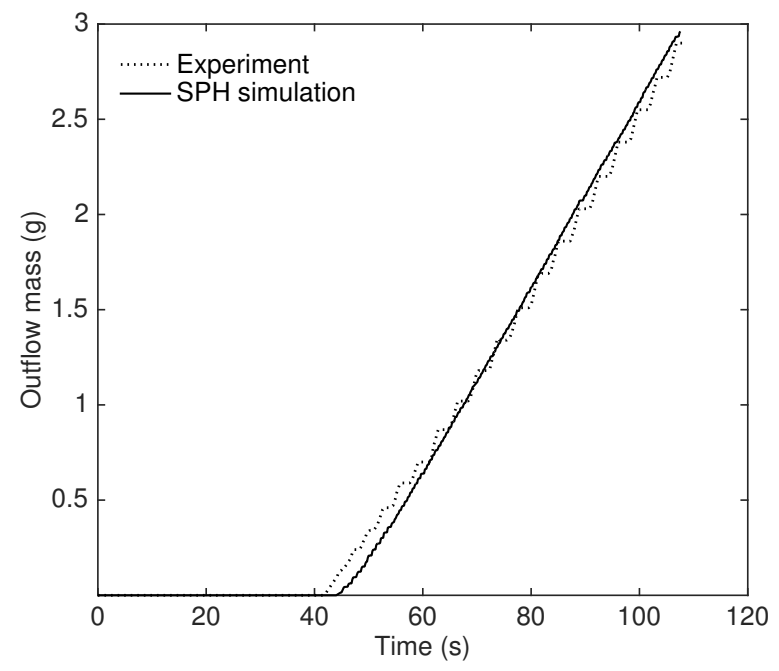

FIGURE 6.10: Experimental measurements and simulation results of water outflow mass.

\subsection{Preferential flow dynamics at a fracture-matrix interface}

The following section is devoted to the numerical investigation of infiltration dynamics at a fracture-matrix interface. Here, we consider two types of vertical fractures, one with a permeable and one with an impermeable matrix. The simulation setup consists of two blocks of solid particles separated by a $2.0 \mathrm{~mm}$ fracture. Each block of solid particles has a width and length of $2.0 \mathrm{~cm}$, and a thickness of $2.0 \mathrm{~mm}$.

The fluid is injected at a $12.0 \mathrm{~mm}$ distance from the fracture top with a constant rate (Fig. 6.11). We consider 12 different injection rates, ranging from $2 \times 10^{-8}$ to $2 \times 10^{-6} \mathrm{~m}^{3} \mathrm{~s}^{-1}$.

The mass and initial density of each solid and fluid particle are $m_{0}=8 \times 10^{-9} \mathrm{~kg}$, $\rho_{0}=1000 \mathrm{~kg} / \mathrm{m}^{3}$, respectively, and the smoothing length is set to $h=6.84 \times 10^{-4} \mathrm{~m}$. In the NS equations, the viscosity is $\mu=1.296 \times 10^{-3} \mathrm{Pas}$, the speed of sound is $c=2.5 \mathrm{~m} / \mathrm{s}$, and the gravitational acceleration is $\mathbf{g}=9.81 \mathrm{~m} / \mathrm{s}^{2}$. In the Richards 


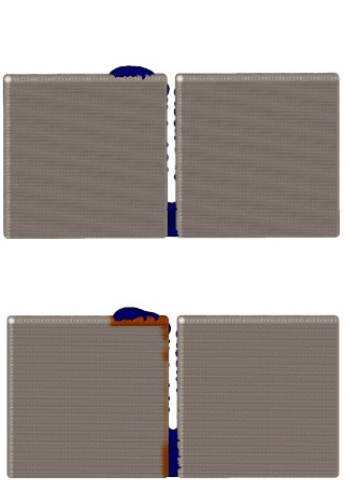

(a)

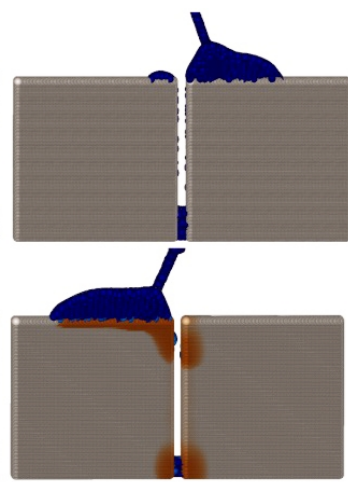

(b)

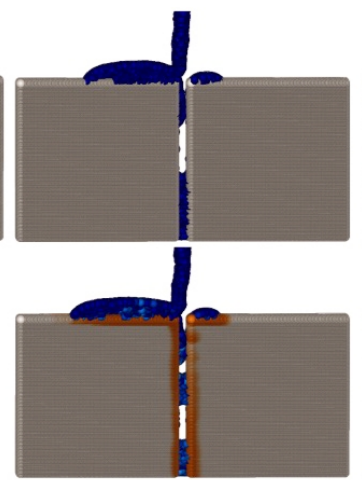

(c)

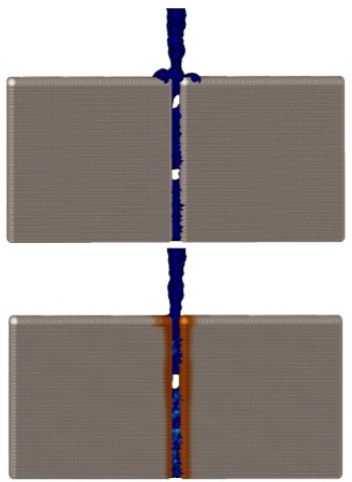

(d)

FIGURE 6.11: Infiltration dynamics in fractures with impermeable (top) and permeable (bottom) walls: (a) $Q=2 \times 10^{-8} \mathrm{~m}^{3} \mathrm{~s}^{-1}, t=$ $4.560 \mathrm{~s}$; (b) $Q=8 \times 10^{-8} \mathrm{~m}^{3} \mathrm{~s}^{-1}, t=0.912 \mathrm{~s}$; (c) $Q=6 \times 10^{-7} \mathrm{~m}^{3} \mathrm{~s}^{-1}$, $t=0.251 \mathrm{~s} ;$ (d) $Q=1 \times 10^{-6} \mathrm{~m}^{3} \mathrm{~s}^{-1}, t=0.228 \mathrm{~s}$.

equation, the parameters $\epsilon_{p}, \mathbf{K}_{s}, S_{s}, \Theta_{s}, \Theta_{r}, \psi_{f}$ and $\psi_{b}$, and the van Genuchten $\alpha, m$, $n$ are the same as described in subsection 6.3.2.

Based on the flux supplied to a fracture, the infiltration process in fractures with impermeable (Fig. 6.11, top) and permeable (Fig. 6.11, bottom) walls can occur according to one of the following scenarios (Fig. 6.12):

(1) For small fluxes $Q<6 \times 10^{-8} \mathrm{~m}^{3} \mathrm{~s}^{-1}$ (Fig. 6.11 a) accumulation of water on the top (so-called, ponding effect) and fracture flow occur simultaneously.

(2) For fluxes $Q$ in the range between $6 \times 10^{-8}$ and $1 \times 10^{-7} \mathrm{~m}^{3} \mathrm{~s}^{-1}$ (Fig. $6.11 \mathrm{~b}$ ) water accumulates on the top of the solid, once enough water has accumulated on the top, preferential/fracture flow occurs. Similar scenario is observed during the laboratory experiment for model validation (Subsection 6.3.4).

(3) For fluxes $Q$ in the range between $1 \times 10^{-7}$ and $8 \times 10^{-7} \mathrm{~m}^{3} \mathrm{~s}^{-1}$ (Fig. $6.11 \mathrm{c}$ ) preferential flow and ponding occur simultaneously.

(4) For large $Q<8 \times 10^{-7} \mathrm{~m}^{3} \mathrm{~s}^{-1}$ (Fig. $6.11 \mathrm{~d}$ ) preferential flow dominates in the system. Once, fracture space is fully occupied by water, ponding occurs.

\subsection{Unsaturated fractured porous medium flow}

In the following section, we study the influence of the fracture wall permeability on arrival time and on the volume of water stored in the porous matrix for different infiltration rates. For the simulations, we create two rough parallel fracture surfaces separated by a $2.0 \mathrm{~mm}$ aperture. Each fracture surface has a width of $5.0 \mathrm{~cm}$, a length of $10.0 \mathrm{~cm}$, and a thickness of $1.0 \mathrm{~cm}$. The roughness of the solid surface is characterized by the Hurst exponent $\zeta$ (Bouchaud et al.; 1990; Shigorina et al.; 2019) and an initial maximum value $\Delta$ for the random displacement from a planar surface. It was shown that $\zeta$ often assumes values of $0.80(5)$ for consolidated rocks (Bouchaud; 1997; Ponson et al.; 2006), however, wider ranges of $0<\zeta<0.9$ have been measured as well (Boffa et al.; 1998; Sahimi; 2011). Here we chose $\zeta=0.75$, and $\Delta=40.0 \mathrm{~mm}$. The rough fracture surfaces are resolved with 6784800 solid particles with a particle spacing of $\Delta x=2.0 \times 10^{-4} \mathrm{~m}$. The amount of fluid particles depends on the flux $Q$ and the simulation duration.

The parameters $m_{0}, \rho_{0}, h, \mu, c, \mathbf{g}, \epsilon_{p}, \mathbf{K}_{s}, S_{s}, \Theta_{s}, \Theta_{r}, \psi_{f}$ and $\psi_{b}$, and the van Genuchten $\alpha, m, n$ are the same as described in subsection 6.4. 


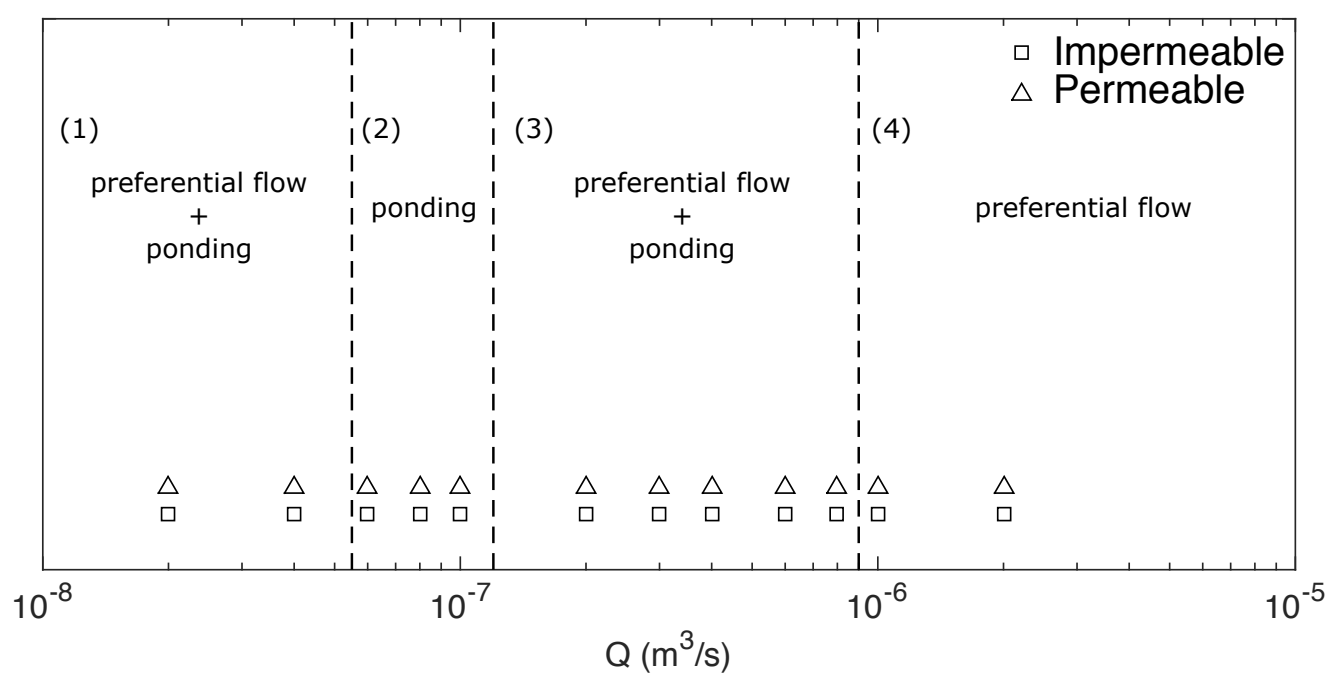

FIGURE 6.12: Four scenarios of infiltration dynamics in fractures with impermeable and permeable walls: (1) for $Q<6 \times 10^{-8} \mathrm{~m}^{3} \mathrm{~s}^{-1}$ preferential flow and ponding occur simultaneously; (2) for $Q$ in the range between $6 \times 10^{-8}$ and $1 \times 10^{-7} \mathrm{~m}^{3} \mathrm{~s}^{-1}$ ponding is dominating; (3) for $Q$ in the range between $1 \times 10^{-7}$ and $8 \times 10^{-7} \mathrm{~m}^{3} \mathrm{~s}^{-1}$ preferential flow and ponding occur simultaneously; (4) for $Q<8 \times 10^{-7} \mathrm{~m}^{3} \mathrm{~s}^{-1}$ preferential flow is dominating.

We consider two types of rough fractures: (1) with impermeable matrix (Fig. 6.13, top) and (2) with permeable matrix $\mathbf{K}_{s}=6.39 \times 10^{-6} \mathrm{~m} \mathrm{~s}^{-1}$ (Fig. 6.13, bottom). Under the term fracture wall we consider a thick porous (permeable or impermeable) matrix adjacent to the fracture void space. The fluid is injected along the fracture top with constant volumetric flux $Q$. Figure 6.13 shows the flow mode distributions inside fractures with impermeable and permeable walls for three infiltration rates: $Q=3 \times 10^{-6} \mathrm{~m}^{3} \mathrm{~s}^{-1}$ (Fig. 6.13a), $Q=9 \times 10^{-6} \mathrm{~m}^{3} \mathrm{~s}^{-1}$ (Fig. 6.13b), and $Q=2 \times 10^{-5} \mathrm{~m}^{3} \mathrm{~s}^{-1}$ (Fig. 6.13c) at arrival times for impermeable fracture. We measure arrival time as a time between fluid injection start and a time when fluid in the fracture void space reaches the bottom of the fracture.

For the smaller infiltration rate $\left(Q=3 \times 10^{-6} \mathrm{~m}^{3} \mathrm{~s}^{-1}\right)$, the dominating flow modes are droplets and a combination between temporary rivulets (slugs, elongated droplets) and snapping droplets (Fig. 6.9a). For the higher flow rate $Q=9 \times 10^{-6} \mathrm{~m}^{3} \mathrm{~s}^{-1}$, we observe a transition into a rivulet-dominated regime with occasional (lateral) merging of rivulets. For even higher flow rates $\left(Q=2 \times 10^{-5} \mathrm{~m}^{3} \mathrm{~s}^{-1}\right)$ flows transitions into snapping films, partially breaking up into rivulets.

Figure 6.13 (bottom) shows the saturation of the porous matrix. In comparison with simulations employing an impermeable matrix (Fig. 6.13, top), a smaller amount of fluid occupies the fracture void space end hence alters the flow-rate dependent formation of flow modes. Figure 6.14 compares the fluid arrival times for for fractures with permeable and impermeable walls. The ratios $t^{*}$ between arrival times for permeable and impermeable matrix systems are listed in Table 6.2.

$$
t *=\frac{t_{i m}}{t_{p}}
$$

where $t_{i m}$ and $t_{p}$ are the arrival times for an impermeable and permeable matrix respectively. As expected the simulation results indicate a delay in arrival time when 

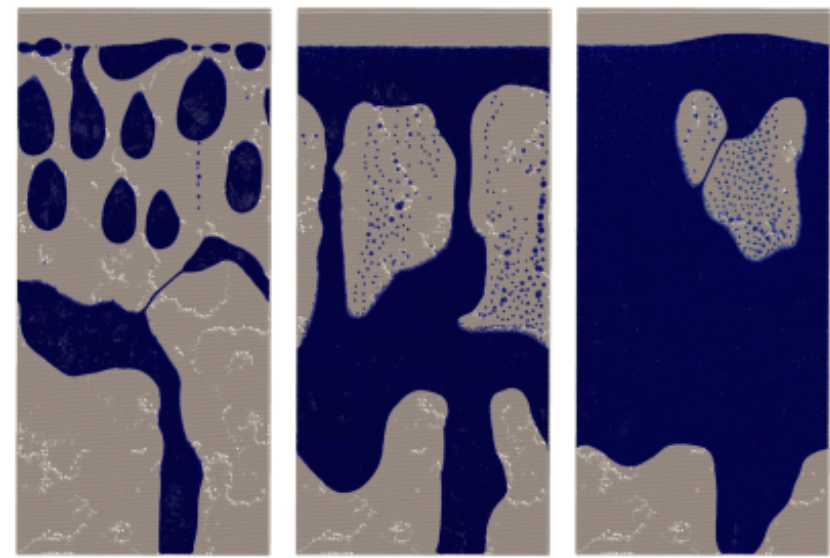

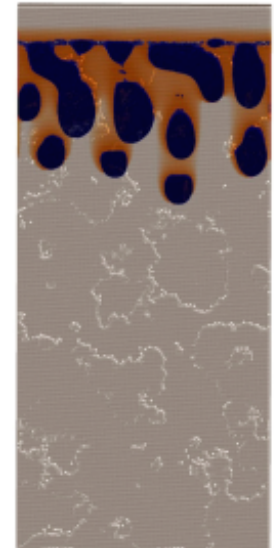

(a)

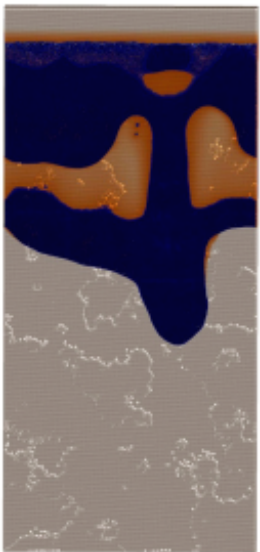

(b)

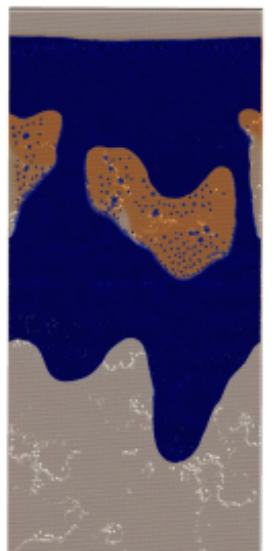

(c)

FIGURE 6.13: Flow mode distributions inside a rough fracture with impermeable (top) and permeable (bottom) walls for different fluxes at arrival times for permeable fracture: (a) $Q=3 \times 10^{-6} \mathrm{~m}^{3} \mathrm{~s}^{-1}, t=$ $1.026 \mathrm{~s}$; (b) $Q=9 \times 10^{-6} \mathrm{~m}^{3} \mathrm{~s}^{-1}, t=0.570 \mathrm{~s}$; (c) $Q=2 \times 10^{5} \mathrm{~m}^{3} \mathrm{~s}^{-1}$, $t=0.388 \mathrm{~s}$

a permeable matrix is present (Fig. 6.14). For an infiltration rate $Q=3 \times 10^{-6} \mathrm{~m}^{3} \mathrm{~s}^{-1}$ we measure a value of $t^{*}=2.11$, i.e., in the case of a permeable fracture matrix the breakthrough is about two times slower than for an impermeable one. For the highest considered infiltration rate $Q=2 \times 10^{-5} \mathrm{~m}^{3} \mathrm{~s}^{-1}$, water is rapidly channeled through the fracture void space to the bottom of the fracture without any significant delay as compared to a fracture with permeable walls.

In order to quantify the amount of water stored in the fracture, we calculate the outflow ratio $\eta$ :

$$
\eta=1-\frac{Q_{o u t}}{Q},
$$

where $Q_{\text {out }}$ is volume of water leaving the system at the bottom of the fracture divided by time and $\eta$ assumes values between 0 and 1 . When $\eta=1$, no fracture outflow occurs and all of the injected water is kept in the porous matrix and within the fracture void space, or on the fracture surface, while a value of $\eta$ close to zero represents a steady state condition, where the outflow rate is equal to the infiltration rate. Figure 6.15 compares changes in $\eta$ over time for an impermeable and permeable matrix. The difference in $\eta$ between impermeable and permeable matrix systems corresponds to the relative amount of water stored in the porous matrix. 


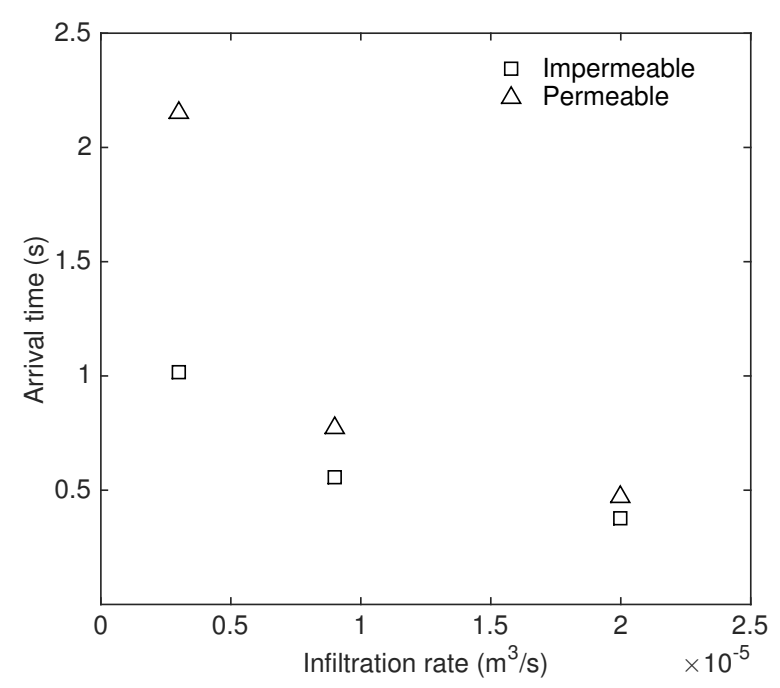

FIGURE 6.14: Dependence of fluid arrival time on infiltration rate for an impermeable and permeable matrix.

Table 6.2 provides the difference in outflow ratio $\Delta \eta$ for an impermeable and permeable matrix at $t=3 \mathrm{~s}$ for different infiltration rates. The largest value of $\Delta \eta_{t=3}=0.71$ occurs at the smallest flux of $Q=3 \times 10^{-6} \mathrm{~m}^{3} \mathrm{~s}^{-1}$, indicating that over $70 \%$ of water is stored within the porous matrix. For larger infiltration rates $Q=9 \times 10^{-6}$ and $Q=2 \times 10^{-5} \mathrm{~m}^{3} \mathrm{~s}^{-1}$, the outflow ratio decrease with $\Delta \eta_{t=3}=0.25$ and 0.18 respectively, i.e., due to limited uptake capacity of the matrix a smaller amount of water infiltrates into the porous matrix.

TABLE 6.2: Statistical properties for different fluxes in rough fractures.

\begin{tabular}{lccc}
\hline $\mathrm{Q}\left(\mathrm{m}^{3} / \mathrm{s}\right)$ & $3 \times 10^{-6}$ & $9 \times 10^{-6}$ & $2 \times 10^{-5}$ \\
\hline$t^{*}$ & 2.11 & 1.38 & 1.24 \\
$\Delta \eta_{t=3}$ & 0.71 & 0.25 & 0.18 \\
\hline
\end{tabular}

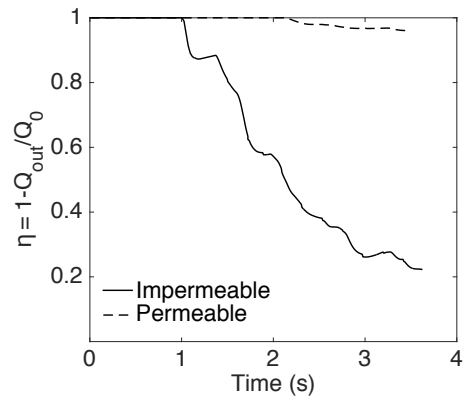

(a)

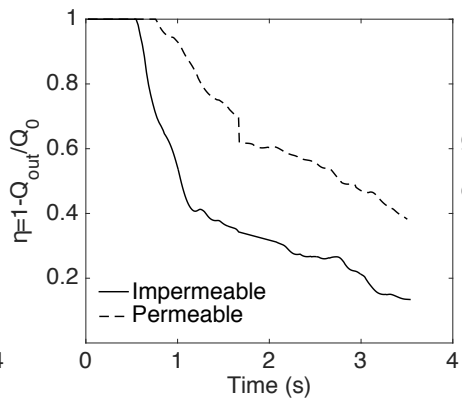

(b)

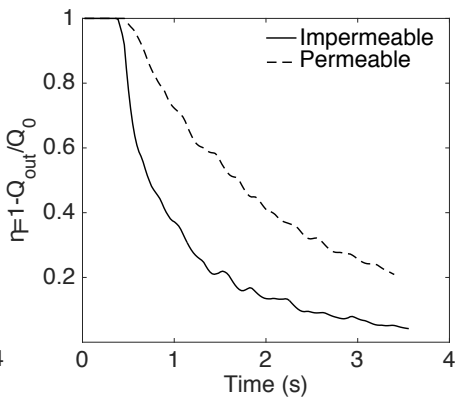

(c)

FIGURE 6.15: The outflow ratio over time for different infiltration rates: (a) $Q=3 \times 10^{-6} \mathrm{~m}^{3} \mathrm{~s}^{-1}$; (b) $Q=9 \times 10^{-6} \mathrm{~m}^{3} \mathrm{~s}^{-1}$; (c) $Q=$ $2 \times 10^{5} \mathrm{~m}^{3} \mathrm{~s}^{-1}$ 


\subsection{Discussion}

In this paper, we present a novel SPH model to investigate preferential flow dynamics in fractures with porous matrix. As expected, we find that flow in fracture is much faster than in matrix. We also find that flow in fracture is affected by the diffuse flow in the adjacent porous matrix.

Specifically, we study infiltration dynamics in a $2.0 \mathrm{~mm}$ fracture for flux rates ranging from $2 \times 10^{-8}$ to $2 \times 10^{-6} \mathrm{~m}^{3} \mathrm{~s}^{-1}$. For a given water inlet location, the infiltration occurs in one of the following four scenarios (see Figs. 6.11 and 6.12). In the first scenario (small infiltration rates $Q<6 \times 10^{-8} \mathrm{~m}^{3} \mathrm{~s}^{-1}$ ), we observe droplet flow in the vertical fracture with some water accumulates on the top horizontal surface. In the second scenario ( $Q$ between $6 \times 10^{-8}$ and $1 \times 10^{-7} \mathrm{~m}^{3} \mathrm{~s}^{-1}$ ), the ponding effect is dominating. In this case, water accumulates first on the top, until saturation is high enough to activate a fracture flow. In the third case ( $Q$ is in the range between $1 \times 10^{-7}$ and $8 \times 10^{-7} \mathrm{~m}^{3} \mathrm{~s}^{-1}$ ), the ponding and fracture flow occur simultaneously. When a water stream reaches the fracture top, it separates into two streams. One of these streams goes into a fracture, another one transmits water to the solid top. In the last scenario $\left(Q<8 \times 10^{-7} \mathrm{~m}^{3} \mathrm{~s}^{-1}\right)$, all water flows into the vertical fracture. If the infiltration rate is high enough to fill all fracture space with water, then water eventually starts accumulating on the horizontal surface. In all these cases, preferential flow transmit water rapidly to the bottom of the fracture, while matrix flow happens slowly even under unsaturated conditions.

Next, we investigate infiltration dynamics in rough fractures with permeable and impermeable walls. We simulate a continuous water flux supplied to the top of a fracture that is $5.0 \mathrm{~cm}$ width, $10.0 \mathrm{~cm}$ long, and has $2.0 \mathrm{~mm}$ aperture and $1.0 \mathrm{~cm}$ wall thickness. The roughness of the fracture walls is characterized by Hurst coefficient $\zeta=0.75$, and $\Delta=40.0 \mathrm{~mm}$. We consider three infiltration rates $Q=3 \times 10^{-6}$, $Q=9 \times 10^{-6}$ and $Q=2 \times 10^{-5} \mathrm{~m}^{3} \mathrm{~s}^{-1}$. The simulation results show a delay in arrival times for a fracture with permeable walls as compared to impermeable walls, especially for $Q=3 \times 10^{-6} \mathrm{~m}^{3} \mathrm{~s}^{-1}$, where due to low free-surface velocities and/or high imbibition capacity, water can efficiently saturate the porous matrix. For the $Q=2 \times 10^{-5} \mathrm{~m}^{3} \mathrm{~s}^{-1}$, water flows rapidly to the bottom of the fracture without any significant delay in arrival time. Under the chosen conditions and depending on the flow rate, the permeable fracture walls can be an efficient storage compartment and store over $70 \%$ of the infiltrated water.

\subsection{Conclusion}

We developed a fully parallelized multiscale SPH model to study infiltration dynamics in porous-fractured rock formations. In our model, flow in porous matrix is governed by the Richards (1931) equation coupled to free-surface flow in the adjacent fracture governed by the Navier-Stokes equation. Inflow dynamics from the fracture into the porous matrix are realized by an efficient particle removal algorithm and a virtual water redistribution formulation in order to enforce mass and momentum conservation. The model is validated via comparison with a numerical COMSOL model and laboratory experiments.

The SPH model for free-surface flow in fractures was proposed and validated in our previous work. To validate the implementation of the Richards equation in the SPH model, we calculated the time-dependent pressure head distribution inside a vertical solid column with a constant pressure head boundary and found a good 
agreement with the corresponding finite element solution obtained with the COMSOL package.

The validation of infiltration dynamics, i.e. imbibition from a free-surface flow domain into the porous matrix is carried out via comparison to three types of smallscale laboratory experiments: (1) droplet imbibition on a horizontal sandstone plate, (2) water column infiltration into a sandstone, and (3) discharge of free-surface flows on a porous medium. For the droplet imbibition experiment, we observe changes in droplet size and shape, and measure the imbibition time. In the second experiment, the time-dependent drawdown of a water column with a total initial volume of $4.0 \mathrm{~mL}$ above a sandstone slice is measured, and in addition the saturation front in the porous matrix visually determined. In the third experiment, we consider a continuous water flux of $3.5 \mathrm{~mL} \mathrm{~min}^{-1}$ supplied to the top right corner of a rectangular sandstone sample. Water accumulates on the top surface and is allowed to discharge across the open right vertical surface. In order to quantify the outflow and interaction of the fluid with the porous matrix, we measure the outflow mass leaving the system and visually determine the saturation of the porous matrix. All laboratory experiments are carried out with Seeberger sandstone samples. The permeability and van Genuchten parameters of the sandstone are estimated from pore size and grain size distribution analysis. Our model is in very good agreement with all considered types of laboratory experiments.

Next, we investigate under which condition preferential flow occurs. Our simulation results show, that a preferential flow occurs simultaneously with a diffuse flow and transmits water much faster, providing a rapid aquifer recharge even under unsaturated and partially saturated conditions. Depending on an infiltration rate and water inlet location, preferential flow or ponding, or both of them can be dominant in the system. For ponding dominating systems, we observe a small delay in fracture flow. In this case, a fracture transmits water when enough water is accumulated on the top of the solid surface. For the preferential flow dominating systems, fracture flow occurs immediately, and ponding occurs only if a fracture space is fully occupied by water.

Finally, we study infiltration dynamics in rough fractures with impermeable and permeable walls. The $2.0 \mathrm{~mm}$ aperture fracture has dimensions of $5.0 \mathrm{~cm}$ width, $10.0 \mathrm{~cm}$ length, and $1.0 \mathrm{~cm}$ wall thickness. The roughness of the fracture walls is characterized by Hurst coefficient. Using our fully coupled numerical model we demonstrate the influence of the fracture wall permeability on the fluid arrival time for different infiltration rates, as well as, on the volume of water which can be stored in the porous fracture walls. A significant influence of the fracture wall permeability we observe in arrival time and in outflow ratio for small infiltration rate.

\section{Acknowledgments}

This work was funded by the Deutsche Forschungsgemeinschaft (DFG; German Research Foundation) under grant KO 5359/1-1 and KO 5359/4-1. A.M. Tartakovsky was supported by the Department of Energy (DOE)'s Office of Advanced Scientific Computing Research at the Pacific Northwest National Laboratory (PNNL). PNNL is operated by Battelle for the DOE under Contract DE-AC05-76RL01830.

\section{Bibliography}

Batchelor, G. K. (1967). An introduction to fluid dynamics, Cambridge University Press, Cambridge, UK.

Bear, J. (2013). Dynamics of fluids in porous media, Courier Corporation. 
Boadu, F. K. (2000). Hydraulic conductivity of soils from grain-size distribution: new models, Journal of Geotechnical and Geoenvironmental Engineering 126(8): 739-746.

Boffa, J. M., Allain, C. and J.P Hulin (1998). Experimental analysis of fracture rugosity in granular and compact rocks, The European Physical Journal Applied Physics 2(3): 281-289.

URL: $h t t p: / / w w w . e p j a p . o r g / 10.1051 / e p j a p: 1998194$

Bouchaud, E. (1997). Scaling properties of cracks, Journal of Physics: Condensed Matter 9(21): 4319.

Bouchaud, E., Lapasset, G. and Planes, J. (1990). Fractal dimension of fractured surfaces: a universal value?, EPL (Europhysics Letters) 13(1): 73.

Buscheck, T. A., Nitao, J. J. and Chestnut, D. (1991). The impact of episodic nonequilibrium fracture-matrix flow on geological repository performance, Technical report, Lawrence Livermore National Lab., CA (United States).

Carman, P. C. (1937). Fluid flow through granular beds, Trans. Inst. Chem. Eng. 15: $150-166$.

DiCarlo, D. A., Bauters, T. W., Darnault, C. J., Steenhuis, T. S., Parlange, J. et al. (1999). Lateral expansion of preferential flow paths in sands, Water Resources Research 35(2): 427-434.

Ganzenmüller, G. C., Steinhauser, M. O., Van Liedekerke, P. and Leuven, K. U. (2011). The implementation of smooth particle hydrodynamics in LAMMPS.

Germann, P., Helbling, A. and Vadilonga, T. (2007). Rivulet approach to rates of preferential infiltration, Vadose Zone Journal 6(2): 207-220.

Ghanbarian-Alavijeh, B., Liaghat, A., Huang, G.-H. and Van Genuchten, M. T. (2010). Estimation of the van genuchten soil water retention properties from soil textural data, Pedosphere 20(4): 456-465.

Guarracino, L. (2007). Estimation of saturated hydraulic conductivity ks from the van genuchten shape parameter $\alpha$, Water Resources Research 43(11).

Hall, H. N. et al. (1953). Compressibility of reservoir rocks, Journal of Petroleum Technology 5(01): 17-19.

Heilweil, V. M., Benoit, J. and Healy, R. W. (2015). Variably saturated groundwater modelling for optimizing managed aquifer recharge using trench infiltration, Hydrological Processes 29(13): 3010-3019.

Kordilla, J., Noffz, T., Dentz, M., Geyer, T. and Tartakovsky, A. M. (2017). Effect of unsaturated flow modes on partitioning dynamics of gravity-driven flow at a simple fracture intersection: laboratory study and three-dimensional smoothed particle hydrodynamics simulations., Water Resources Research 53(11): 9496-9518.

Kordilla J., Tartakovsky A.M., G. T. (2013). A smoothed particle hydrodynamics model for droplet and film flow on smooth and rough fracture surfaces, Advances in Water Resources 59: 1-14.

Kozeny, J. (1927). Uber kapillare leitung der wasser in boden, Royal Academy of Science, Vienna, Proc. Class I 136: 271-306. 
Lee, J., Radu, A., Vontobel, P., Derome, D. and Carmeliet, J. (2016). Absorption of impinging water droplet in porous stones, Journal of colloid and interface science 471: 59-70.

Mandelbrot, B. B. (1983). The fractal geometry of nature, Vol. 173, WH freeman New York.

Marmur, A. (1988). Drop penetration into a thin porous medium, Journal of Colloid and Interface Science 123(1): 161-169.

Morris, J. P., Fox, P. J. and Zhu, Y. (1997). Modeling low Reynolds number incompressible flows using SPH, Journal of Computational Physics 136(1): 214-226.

Nimmo, J. R. (2010). Theory for source-responsive and free-surface film modeling of unsaturated flow, Vadose Zone Journal 9: 295-306.

Nimmo, J. R. (2012). Preferential flow occurs in unsaturated conditions, Hydrological Processes 26(5): 786-789.

Nitao, J. J. (1991). Theory of matrix and fracture flow regimes in unsaturated, fractured porous media, Technical report, Lawrence Livermore National Lab., CA (United States).

Plimpton, S. (1995). Fast parallel algorithms for short-range molecular dynamics, Computational Physics 117(1): 1-19.

Ponson, L., Bonamy, D. and Bouchaud, E. (2006). Two-dimensional scaling properties of experimental fracture surfaces, Physical Review Letters 96(3): 035506.

Richards, L. A. (1931). Capillary conduction of liquids through porous mediums, Physics 1(5): 318-333.

Sahimi, M. (2011). Flow and transport in porous media and fractured rock: from classical methods to modern approaches, John Wiley \& Sons.

Shigorina, E., Kordilla, J. and Tartakovsky, A. M. (2017). Smoothed particle hydrodynamics study of the roughness effect on contact angle and droplet flow, Physical Review E 96(3): 033115.

Shigorina, E., Tartakovsky, A. M. and Kordilla, J. (2019). Investigation of gravitydriven infiltration instabilities in smooth and rough fractures using a pairwiseforce smoothed particle hydrodynamics model, Vadose Zone Journal 18(1).

Siregar, D. P. (2012). Numerical simulation of evaporation and absorption of inkjet printed droplets.

Sustrate, A.-M. (2017). Strömungsexperimente in ungesättigten porös-geklüfteten medien: Interaktion zwischen freier oberflächenströmung und poröser matrix, Master's thesis, Georg-August-Universität Göttingen.

Tartakovsky, A. M. and Meakin, P. (2005a). A smoothed particle hydrodynamics model for miscible flow in three-dimensional fractures and the two-dimensional rayleigh-taylor instability, Journal of Computational Physics 207(2): 610-624.

Tartakovsky, A. M. and Panchenko, A. (2016). Pairwise force smoothed particle hydrodynamics model for multiphase flow: surface tension and contact line dynamics, Journal of Computational Physics 305: 1119-1146. 
Tartakovsky, A. and Meakin, P. (2005b). Modeling of surface tension and contact angles with smoothed particle hydrodynamics, Physical Review E 72(2).

Therrien, R. and Sudicky, E. (1996). Three-dimensional analysis of variably-saturated flow and solute transport in discretely-fractured porous media, Journal of Contaminant Hydrology 23(1-2): 1-44.

Tyler, S. W. and Wheatcraft, S. W. (1992). Fractal scaling of soil particle-size distributions: analysis and limitations, Soil Science Society of America Journal 56(2): 362-369.

Van Genuchten, M. T. (1980). A closed-form equation for predicting the hydraulic conductivity of unsaturated soils 1, Soil Science Society of America Journal 44(5): 892 898.

Wendland, H. (1995). Piecewise polynomial, positive definite and compactly supported radial functions of minimal degree., Advances in Computational Mathematics 4(1): 389-396. 



\section{Chapter 7}

\section{Summary and conclusion}

This chapter provides an overview of the main research results, and gives suggestions for further investigations. The objectives of this thesis are the investigation of preferential flow dynamics in dry, partially saturated, and saturated porousfractured media at core- and micro scale, and the development of a comprehensive numerical SPH model for their quantitative and qualitative simulations.

\subsection{Main results}

Chapters 4,5 , and 6 cover the main research work of this thesis.

\subsubsection{The effect of roughness on contact angle and droplet flow}

Chapter 4 studies the effect of surface roughness and type of surface (hydrophobic or hydrophilic) on the effective contact angle and droplet dynamics. The main results of this investigation are the following:

- The effective static contact angles of Cassie (Cassie and Baxter; 1944) and Wenzel (Wenzel; 1936) droplets on a rough surface are greater than the corresponding static contact angles on a smooth surface. As a result, micro-scale hydrophobic rough surfaces also show effective hydrophobic behavior. On the other hand, micro-scale hydrophilic surfaces may be macroscopically hydrophilic or hydrophobic, depending on the type of roughness.

- The transition between Cassie and Wenzel states of droplets depends on the roughness and droplet size, which can be linked to the critical pressure for the given fluid-substrate combination. We observe good agreement between simulations and theoretical predictions.

- The investigation of droplet dynamics depending on roughness orientation (i.e., an anisotropic roughness) and surface inclination shows that droplet flow velocities are lower if the surface roughness is oriented perpendicular to the flow direction. If the predominant elements of surface roughness are in alignment with the flow direction, the flow velocities increase compared to smooth surfaces, which can be attributed to the decrease in fluid-solid contact area similar to the lotus effect.

- The classical linear scaling relationships (Podgorski et al.; 2001) between Bond and capillary number for droplet flow on flat surfaces also hold for flow on rough surfaces. 


\subsubsection{Infiltration instabilities in smooth and rough fractures}

Chapter 5 investigates fracture-specific infiltration dynamics in smooth and rough single fractures. Three types of fractures are considered with different degrees of roughness, including a smooth fracture. This work studies the influence of random fracture roughness (Bouchaud et al.; 1990) and injection rate on fluid flow modes and flow velocity. To characterize the flow instability, we calculate standard deviations of velocity along the fracture width.

The main results of this research are:

- Both the rough and smooth fractures exhibit flow instabilities, fingering, and intermittent flow regimes for low infiltration rates.

- In agreement with theoretical predictions (Nicholl et al.; 1994), a flat fluid front is achieved when the flux $q$ supplied to a fracture is larger than the gravitationally driven saturated flux $(q>k \rho \mathrm{g} / \mu \cos \varphi)$.

- An increase in degree of roughness corresponds to a decrease in flow velocity and an increase in standard velocity deviation. This is caused by a higher likelihood of flow discontinuities in the form of fingering and/or snapping rivulets.

- The scaling estimation of specific discharge with normalized finger velocity and the relationship between fingertip length and scaled finger velocity show a good agreement with experimental results (Nicholl and Glass; 2005), confirming the validity of the code even under such complex conditions.

\subsubsection{The development of multiscale SPH model for flow in fractured- porous media}

Chapter 6 presents a newly developed multiscale SPH model which allows to simulate flow within a continuum-type porous matrix governed by the Richards (1931) equation and discrete free-surface flows governed by the Navier-Stokes equation. This work is focused on the investigation of preferential flow dynamics in fractures and at a fracture-matrix interface.

The results of this research are:

- The validation examples of a newly developed SPH model are in a good agreement with finite element COMSOL simulations and laboratory experiments.

- The simulation results show, that preferential flow occurs simultaneously with a diffuse flow and transmits water much faster, providing rapid aquifer recharge even under unsaturated and partially saturated conditions.

- A significant influence of the fracture wall permeability is observed in arrival time and in outflow ratio especially for small infiltration rate.

\subsection{General conclusion}

In order to provide an innovative numerical tool for the investigation of rapid recharge in fractured aquifers an PF-SPH model for open-surface, porous-medium and fracture flow has been developed and implemented to simulate infiltration dynamics in smooth and rough fractures and across a fracture-matrix interface. The model can successfully recover the effects of preferential flow pathways for various wetting 
conditions and static and dynamic fluid contact angles. The newly developed fully coupled SPH model discretizes the Navier-Stockes equation for fracture flow and Richards (1931) equation for porous-medium flow and can predict the fluid arrival time in dry, partially saturated or fully saturated fractured porous aquifers.

The simulation results show that preferential flow occurs under various unsaturated, partially-saturated and saturated conditions, providing a rapid water transmission from the top to the bottom of the fracture. Based on the infiltration rate different flow modes can be formed ranging from tiny adsorbed films and droplets to rivulets and thick (wavy) films. Preferential flow dynamics is characterized by the flow instability, fingering, irregular wetting fronts and irregular transitions between flow modes, which makes it difficult to generalize infiltration processes. The effect of fracture/surface roughness on average flow velocity is rather complex under most conditions. For some types of roughnesses and its orientation, the average flow velocity on such surfaces can be higher than the corresponding flow velocity on a smooth surface, but mostly the "natural" surface/fracture roughness decreases the flow velocity, and increases the irregularity/ instability of a fluid front. In contrast to the statement, that preferential flow occurs only for high infiltration rates or saturated porous systems, the SPH simulations provide examples for preferential flow occurring for all infiltration rates, even for unsaturated permeable-porous-fractured rocks. For all infiltration rates and fracture roughness configurations, the preferential flow velocity is much higher than the diffuse flow velocity, even if diffuse flow dominates the system.

The applications of the SPH model are limited to small-scale (maximum $\approx 1.0 \mathrm{~m}^{3}$ ) simulations. Large scales require coarser resolution, which is not suitable for the discretization of tiny fracture spaces. Furthermore, the discretization of infiltration processes at a large scale with fine resolution requires high computational power, which is not possible with most available computational resources. In order to provide a reliable tool for the investigation of infiltration dynamics at a large field scale, the existing SPH model requires further development and possible convergence with upscaled methods.

\subsection{Suggestions for further research}

\subsubsection{Adaptive resolution}

The developed PF-SPH model can successfully simulate gravity-driven flow in fractures and the adjacent porous matrix, including the effects of preferential pathways, and flow mode distributions in smooth and rough fractures. The main research of this thesis is carried out on a micro scale $\approx 1.0 \mathrm{~cm}^{3}$ (Chapter 4 ), and on a core scale $\approx 100.0 \mathrm{~cm}^{3}$ (Chapters 5 and 6 ). For the studied systems and given static resolution, the Courant-Friedrichs-Lewy (CFL) conditions of the explicit time stepping algorithm limit simulation to about 3.0 - 5.0 min real time.

However, many industrial problems, such as water supply, waste repositories, $\mathrm{CO}_{2}$ storage, fracking are associated with large aquifers, reservoirs, and require large-scale and long term simulations. The application of the developed PF-SPH model on larger scales is computationally challenging. Due to the strong contrast between fracture void space and pore throats, an adaptive resolution algorithm would decrease computation times. In order to capture all effects of preferential flow, the fracture space can be resolved with a finer resolution, while porous medium flow can be successfully resolved at a coarser resolution. An adaptive resolution in principle only requires particle-dependent sizes of $h$ (and modifications of the kernel 
computation when particles of different size interact) in order to account for variable particle number densities (volumes), however, this task can be challenging for free-surface flows in the presence of surface tension.

\subsubsection{Implementation of an evapotranspiration equation}

The main research of this thesis deals with the flow in unsaturated zone, therefore in direct contact with the atmosphere. Eqs.(3.17)-(3.18) do not consider any fluid losses due to evaporation. For the implementation of the evapotranspiration equation additional parameters (such as, temperature, humidity, etc.) must be taken into account. In principal a similar algorithm as for the the particle removal (fracturematrix interaction) can be employed. The validation can be carried out via comparison to small-scale laboratory experiments. However, this process acts on rather large time scales and may be out of reach under certain conditions.

\subsubsection{Particle addition algorithm}

For the fracture-matrix interaction a particle removal algorithm was developed, which is suitable for the saturation of dry or partially-saturated soils, but does not take into account water outflow through the lateral boundary or water migration from the porous matrix back into the fracture (e.g., dripping processes). This task requires implementation of additional LAMMPS classes and variable arrays, as well as validations via laboratory experiments.

\subsubsection{Multiphase flow simulations}

Many applications of flow in unsaturated fractured porous media not only deal with one fluid phase. For example, $\mathrm{CO}_{2}$ or waste storage problems involve two or more fluid phases.

In principle, this task requires the implementation of pair-wise forces for additional phases, added in the SPH form of the momentum conservation equation. The magnitude of these forces depends on respective wetting properties and surface tension of the respective phase. SPH multiphase flow simulations were, for example, shown by Tartakovsky et al. (2016), where pressure gradient in the momentum conversation equation is commonly evaluated from the number density to avoid restrictions due to strong mass density differences.

\subsubsection{Reactive transport}

The current model SPH model discretizes the Navier-Stokes equation and does not consider (reactive) mass transport processes. However, the simulation of reactive transport has, for example, been shown by Kordilla et al. (2014); Tartakovsky and Panchenko (2016); Tartakovsky et al. (2016), and in general requires the implementation of chemical reaction and transport between different particle types (e.g., fluid to fluid or fluid to solid) and allows to simulate complex precipitation phenomena.

\section{Bibliography}

Bouchaud, E., Lapasset, G. and Planes, J. (1990). Fractal dimension of fractured surfaces: a universal value?, EPL (Europhysics Letters) 13(1): 73. 
Cassie, A. and Baxter, S. (1944). Wettability of porous surfaces, Transactions of the Faraday Society 40: 546-551.

Kordilla, J., Pan, W. and Tartakovsky, A. (2014). Smoothed particle hydrodynamics model for landau-lifshitz-navier-stokes and advection-diffusion equations, The Journal of Chemical Physics 141(22): 224112.

Nicholl, M., Glass, R. and Wheatcraft, S. (1994). Gravity-driven infiltration instability in initially dry nonhorizontal fractures, Water Resources Research 30(9): 2533-2546.

Nicholl, M. J. and Glass, R. J. (2005). Infiltration into an analog fracture: Experimental observations of gravity-driven fingering, Vadose Zone Journal 4: 1123-1151.

Podgorski, T., Flesselles, J.-M. and Limat, L. (2001). Corners, cusps, and pearls in running drops, Physical Review Letters 87(3): 036102.

Richards, L. A. (1931). Capillary conduction of liquids through porous mediums, Physics 1(5): 318-333.

Tartakovsky, A. M. and Panchenko, A. (2016). Pairwise force smoothed particle hydrodynamics model for multiphase flow: surface tension and contact line dynamics, Journal of Computational Physics 305: 1119-1146.

Tartakovsky, A. M., Trask, N., Pan, K., Jones, B., Pan, W. and Williams, J. R. (2016). Smoothed particle hydrodynamics and its applications for multiphase flow and reactive transport in porous media, Computational Geosciences 20(4): 807-834.

Wenzel, R. N. (1936). Resistance of solid surfaces to wetting by water, Industrial $\mathcal{E}$ Engineering Chemistry 28(8): 988-994. 